\title{
A nuclear export sequence in Nup214 promotes its targeting to the nuclear pore complex
}

\author{
Dissertation \\ for the award of the degree \\ "Doctor rerum naturalium" \\ of the Georg-August-Universität Göttingen
}

within the doctoral program "Molecular Biology of Cells"

of the Georg-August University School of Science (GAUSS)

submitted by

Mohamed Hamed

from Cairo

Göttingen 2020 

Thesis Committee / Examination Board

Prof. Dr. Ralph H. Kehlenbach $\left(1^{\text {st }}\right.$ referee)

Department of Molecular Biology

University Medical Center Göttingen

Georg-August-Universität Göttingen

Prof. Dr. Heike Krebber ( $2^{\text {nd }}$ referee)

Department of Molecular Genetics

Institute for Microbiology and Genetics

Georg-August-Universität Göttingen

Dr. Achim Dickmanns

Department of Molecular Structural Biology

Georg-August-Universität Göttingen

Further members of the Examination Board

Prof. Dr. Michael Thumm

Department of Cell Biochemistry

University Medical Center Göttingen

Georg-August-Universität Göttingen

Prof. Dr. Michael Meinecke

Department of Cellular Biochemistry

University Medical Center Göttingen

Dr. Sigrid Hoyer-Fender

Department of Developmental Biology

Georg-August-Universität Göttingen

Date of oral examination: 



\section{Statutory declaration}

I hereby declare that I have written this $\mathrm{PhD}$ thesis independently with no other sources and aids than clearly stated.

This thesis was not submitted in the same or in a substantially similar version to any other authority to achieve an academic grading and was not published elsewhere.

Göttingen, February 2020

Mohamed Hamed 



\section{Table of Contents}

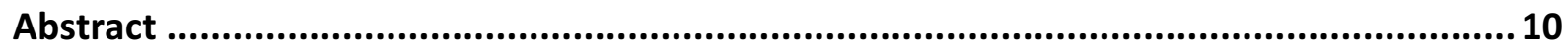

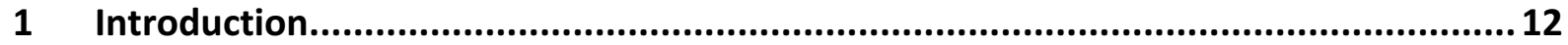

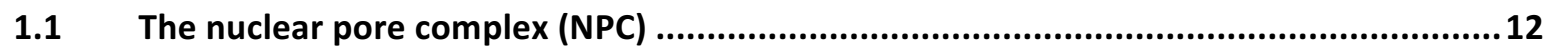

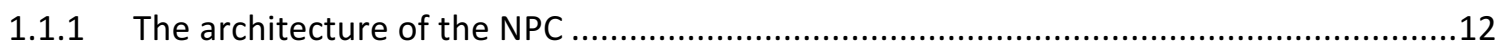

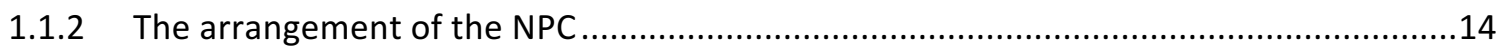

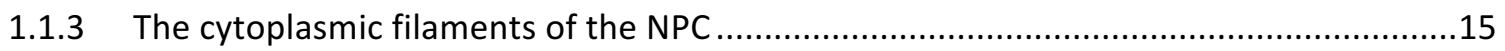

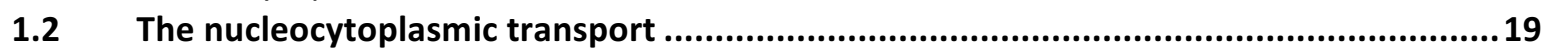

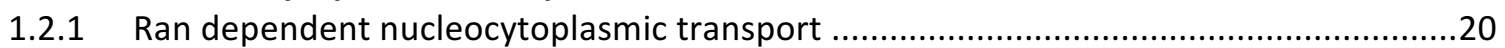

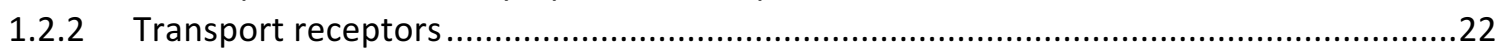

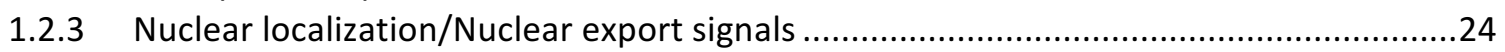

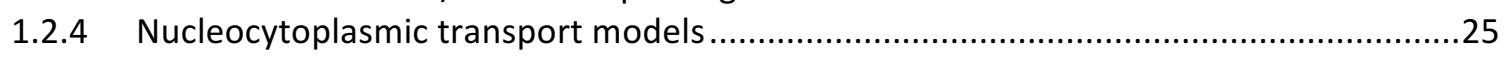

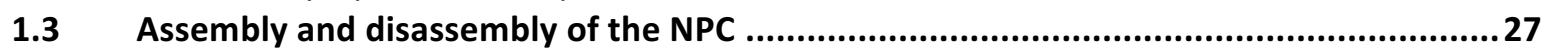

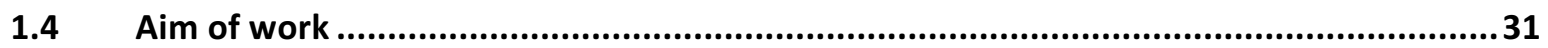

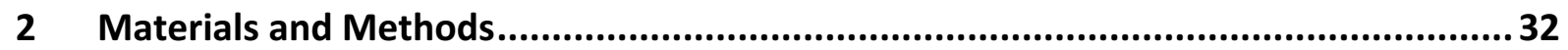

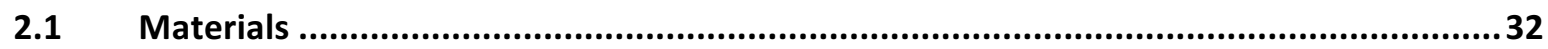

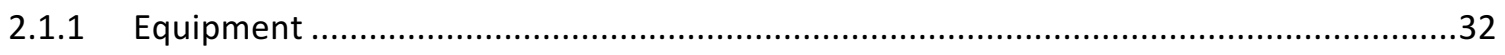

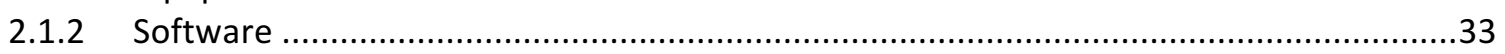

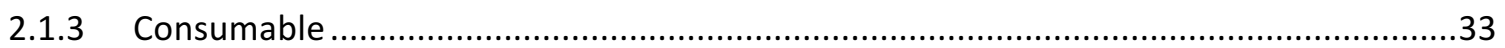

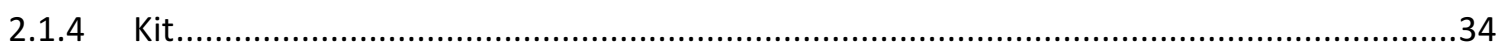

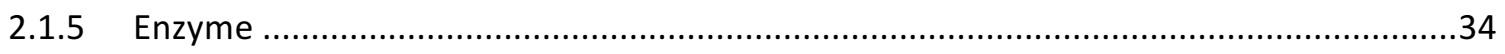

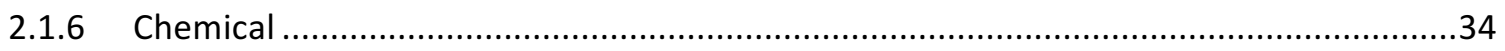

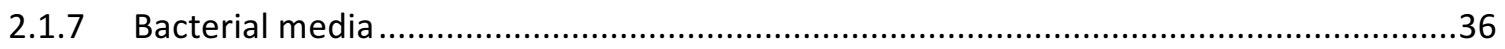

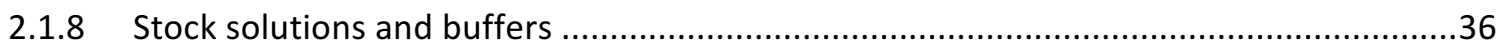

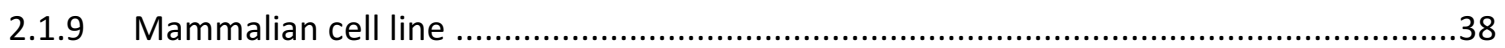

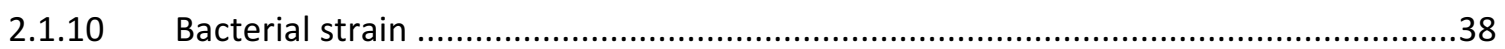

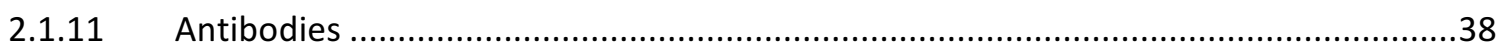

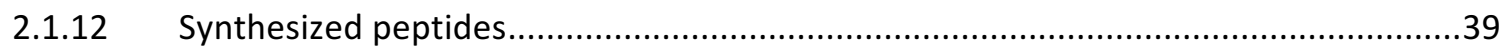

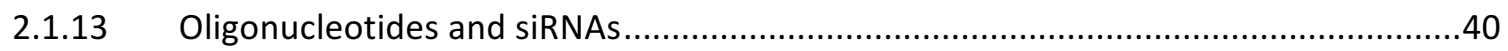

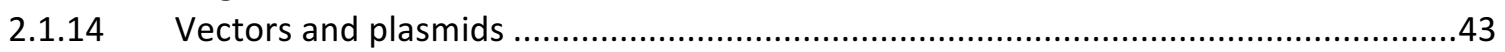

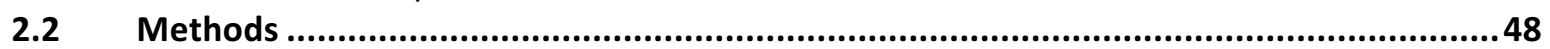

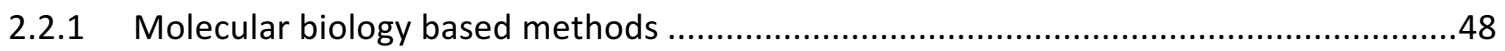

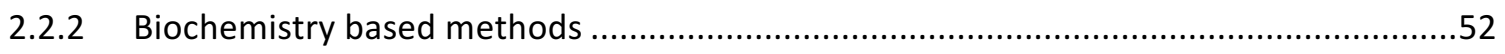

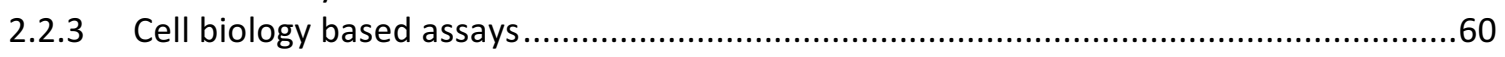

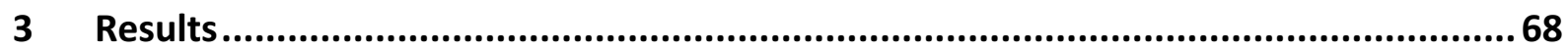

3.1 A nuclear export sequence in Nup214 promotes its targeting to the nuclear pore

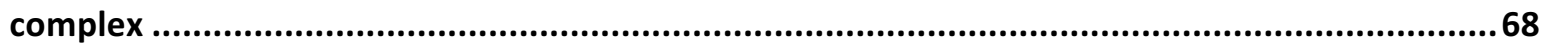

3.1.1 Expression of Nup214 results in cytoplasmic/ Nuclear envelope localization ................68

3.1.2 Nup214 has a nuclear export signal in its coiled coil region.............................................

3.1.3 The Nup214 NES sequence is conserved across different species ...................................72

3.1.4 LMB resistant CRM1 localizes GFP-Nup214 back to the cytoplasm in LMB treated cells73

3.1.5 Regions other than the FG-repeat can give Nup214 access to the nucleus ....................74

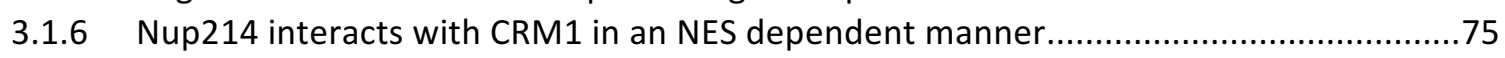

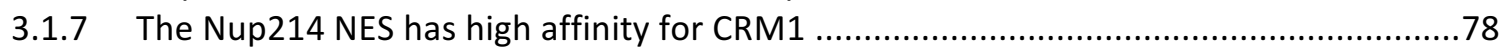

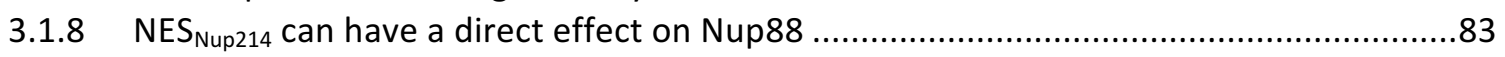

3.1.9 Adding an NES sequence to Nup214 mutant can rescue the Nup214 phenotype in cells ..

3.1.10 Nup358 may play a critical role in helping the insertion of Nup214 into the nuclear envelope. 
3.1.11 Overexpression of Nup214 can restore Nup88 in Nup214 depleted cells ................95

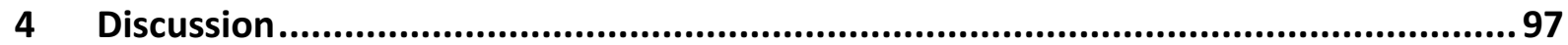

4.1 Different regions of Nup214 show differential localization patterns.............................97

4.2 Nup214 binds CRM1 in a RanGTP dependent manner ...........................................98

4.3 The NES of Nup214 has a high affinity for CRM1..................................................99

4.4 The Nup88-Nup214 subcomplex localizes within the cell in an NES dependent manner 100

4.5 An artificial NES sequence at the C-terminus of Nup214 could form a CRM1 dependent

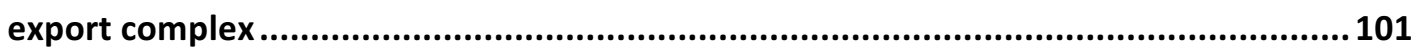

4.6 The Nup214-NES may play a role in targeting Nup214/Nup88 complex in a Nup358

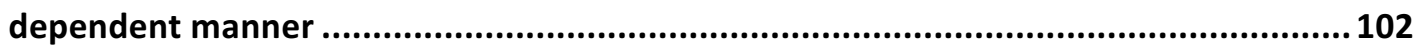

4.7 A model for NES-dependent targeting of Nup214 to the NPC .......................................104

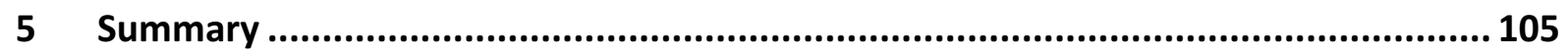

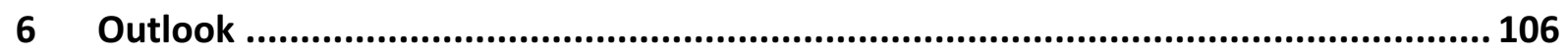

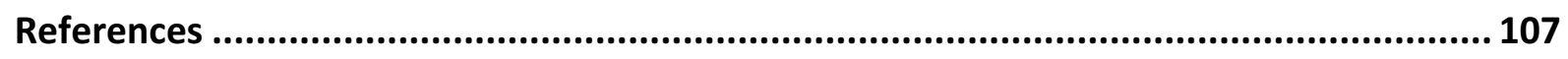

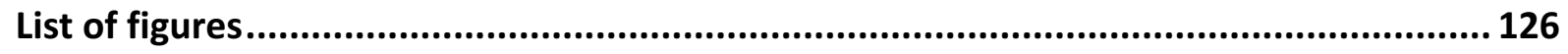

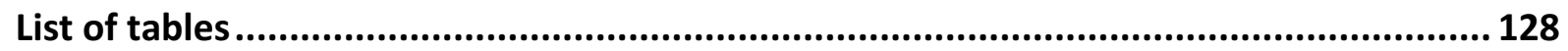

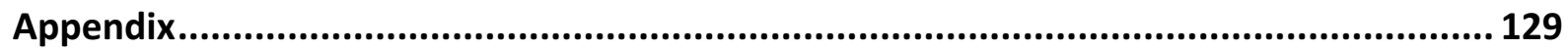

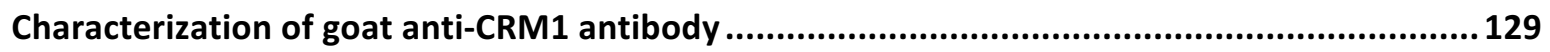

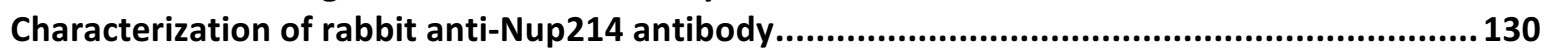

Characterization of cell lines stably expressing GFP-Nup214 (L878A/L882A)..........................132

Supplementary figures ............................................................................................... 134

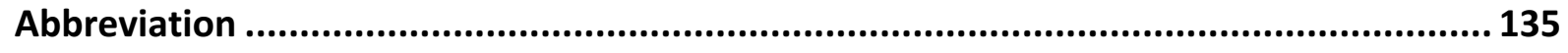

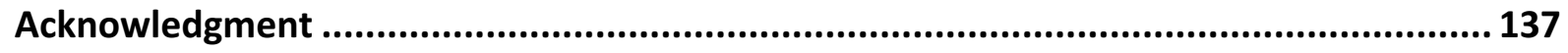

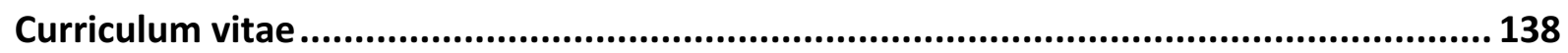





\section{Abstract}

The nuclear pore complex (NPC) controls the communication between the nucleus and the cytoplasm in a selective manner via a hydrophobic barrier involving phenylalanine-glycine (FG) repeats. Interactions with components of this barrier initiate the transport across the NPC. Nucleoporins are the building unit of the NPC. The NPC is composed of multiple copies of 30 different nucleoporins, which have a defined localization pattern along the nuclear pore complex. Nup214 and Nup88 are two nucleoporins, which exist as a subcomplex at the cytoplasmic face of the nuclear pore.

CRM1 is the main nuclear export receptor of the importin $\beta$ superfamily that binds to nuclear export sequences (NESs) and mediates the transport of a large number of proteins across the NPC. The crystal structure of a C-terminal fragment of Nup214 and a trimeric export complex containing CRM1, RanGTP and an export cargo points to a specific role of hydrophobic patches in CRM1 that interact with FG-repeats of the nucleoporin.

In an intact cell, Nup214 and its binding partner Nup88 exist as a complex. While Nup214 is a FG-nucleoporin, Nup88 does not have FG repeats. The two proteins were found to be interdependent and the presence of Nup88 is highly dependent on Nup214 with previous results showing loss of Nup88 upon depletion of Nup214, but not the other way around.

Interestingly, a putative NES region in Nup214 was identified, which is in close proximity to the Nup88-binding site. Using fluorescence microscopy, the effect of the NES on the localization of Nup214 and Nup88 was investigated. In this context, coordinated overexpression studies of Nup214 and its fragments/mutants with Nup88 were performed. Additionally, biochemical assays were used to form a CRM1/RanGTP-dependent complex with Nup214 NES and FG-repeat fragments. Furthermore, the affinity of Nup214 NES was tested and compared to previously published NESs. RNAi experiments were performed to investigate the role of Nup358 in incorporation of Nup214 into the NPC.

The results revealed that Nup214 contains an NES which has a high binding affinity for CRM1. Additionally, Nup214 can interact with CRM1 in a RanGTP dependent manner and localizes to the cytoplasm and at the nuclear envelope (NE). The CRM1 inhibitor, LMB, shifted the localization of tagged versions of Nup214 to the nucleus and a mutation in the Nup214 NES sequence led to similar results. Co-immunoprecipitation experiments showed that Nup214 and Nup88 can interact independently of the mutations in the NES region of Nup214. Furthermore, knockdown of Nup214 resulted in loss of endogenous Nup88, but the endogenous Nup88 was rescued by re-expression of Nup214. Finally, Nup358 depletion resulted in loss of 
overexpressed Nup214 at the NE. This study suggests a model of CRM1- and possibly Nup358dependent Nup214 incorporation into the NPC. 


\section{Introduction}

\subsection{The nuclear pore complex (NPC)}

The cellular processes are highly regulated by various mechanisms. The cell has to ensure that transport occurring across its membranes is tightly controlled. Eukaryotic cells have developed to form a separation of their own genetic material from the remaining compartments in the cell. This serves as a protection of the genetic information carried on the DNA. The nuclear envelope (NE) encloses the genetic information within the nucleus of the cell. A very prominent example that reflects the importance of this partitioning is the separation between the mRNA transcription machinery and the translation/post-translation machinery. However, for this separation to take place it is important for the cell to regulate the movement of the RNAs and proteins in and out of the nucleus. Not all proteins that get translated in the cytoplasm will be active there. In fact, many proteins perform their functions only in the nucleus. Additionally, the nucleus does not have the machinery required for protein synthesis, thus it has to import its proteins from the cytoplasm (Bonner, 1975). The scale of complexity of transport can increase when bigger complexes involving mRNA-bound proteins are transported. For the cell to control the transport across its NE, it needs the very tightly regulated gateway, the NPC.

Eukaryotic cells require the continuous shuttling of molecules between the cytoplasm and nucleus to control gene expression, cell growth and cell division. In addition to its role in nucleocytoplasmic transport, the NPC function was linked to other cellular processes. These include regulation of transcription, development of transcriptional memory and organization of chromatin (reviewed in Light \& Brickner, 2013). Furthermore, the NPC was associated with organizing complexes in close proximity to the nuclear periphery which has a role in assembling messenger ribonucleoproteins (mRNPs) before exporting them (Montpetit et al., 2011). Additionally, the importance of Nups in regulation of the cell cycle and NPC biogenesis was investigated closely (reviewed in Hetzer et al., 2005).

\subsubsection{The architecture of the NPC}

The overall structure of the NPC is conserved between different species. The average size of a yeast NPC is around $60 \mathrm{kDa}$ (Rout et al., 2003) while that of vertebrates is nearly $120 \mathrm{kDa}$ (Reichelt et al., 1990; Cronshaw et al., 2002). The NPC is one of the biggest complexes that exist in the cell (Tamura \& Hara-Nishimura, 2013). The NPC is a toroidal shaped complex (Bui et al., 2013) which has a height of $80 \mathrm{~nm}$, a diameter of 120-145 nm and a transport channel of 
roughly 25 nm (Jarnik \& Aebi, 1991; Goldberg \& Allen, 1992; Cordes et al., 1993; Ris, 1997; Winey et al., 1997; Adam et al., 1990; Lin et al., 2016) (Figure 1).

One important function of the NPC is to stabilize the NE by fusing both the inner and outer nuclear envelopes together (Cronshaw et al., 2002). The NE has an array of NPCs embedded in it (Maul \& Deaven, 1977). Even though a single NPC is made up of several subunits, only 30 distinctive proteins really form the NPC (Maul \& Deaven, 1977; Zwerger et al., 2016; Lin \& Hoelz, 2019). These unique proteins are called nucleoporins (Nups) (Zwerger et al., 2016). The NPC has an eightfold rotational symmetry arrangement following a very specific pattern (Wolf \& Mofrad, 2008; Lin et al., 2016; Lin \& Hoelz, 2019). The NPC has a group of asymmetrically localized Nups on the cytoplasmic side forming the cytoplasmic filaments (Middleton et al., 1984) and another group on the nuclear side forming the nuclear basket. The cytoplasmic side of the NPC includes Nup62, Nup88, Nup214 and Nup358 (Cordes et al., 1993; Fornerod et al., 1995; Fornerod et al., 1997a; Walther et al., 2002), whereas the nuclear side of the NPC contains Tpr, Nup50 and Nup153 (Cordes et al., 1993; Arlucea et al., 1998).

So far, a crystal structure of a complete NPC is not available. As mentioned above, the nucleoporins are organized into stable subcomplexes. Studies were conducted to understand the interactions between these neighboring subcomplexes. The Y-complex (also known in humans as Nup107 complex and in yeast as Nup84 complex) is a well-studied module (Denning et al., 2003). Additional modules were characterized in details, some examples include the Nup62 complex (also known in yeast as the nucleo skeletal like 1 (Nsp1) complex), the Nup93 complex (also known in yeast as the Nup-interacting component of $96 \mathrm{kDa}$ (Nic96) complex) and the Nup214 complex (also known in yeast as the Nup82 complex) (Grandi et al., 1995; Belgareh et al., 1998; Amlacher et al., 2011; Ulrich et al., 2014). Separate reports showed biochemical and structural data about various domains of Nup214 and its binding partner Nup88, however little is known about their role in respect to the overall structure of the NPC as well as their assembly mechanism. Nevertheless, combination of advanced cryo-electron microscopy (cryoEM) techniques (reviewed in Beck \& Baumeister, 2016), cross-linking and mass spectrometry data (Kosinski et al., 2016) and biochemical assembly of subcomplexes (Fischer et al., 2015; Lin et al., 2016) have allowed for the generation of a human NPC map (Bui et al., 2013; reviewed in Beck \& Baumeister, 2016). Cryo-EM accompanied by fluorophore counting, super-resolution microscopy, and mass-spectroscopy were used to analyze purified NE containing intact NPCs. The combination of these techniques revealed that Nups can form heteromeric interactions and that Nup subcomplexes can exist as multiple units in the NPC (Maimon et al., 2012; Mahamid et al., 2016). Recently, the molecular environment of the NPCs 
in relation to the cytoskeletal structures, the lamin network and the nuclear membranes, has been described using enhanced cryo-EM techniques (Mahamid et al., 2016). This helped to understand the flexible interactions between the domains of the subcomplexes of the NPC. Mass spectroscopy data combined with cryo-EM characterized the interactions between the individual members of the Y-complex and the Nup93 complex (Von Appen et al., 2015; Kosinski et al., 2016).

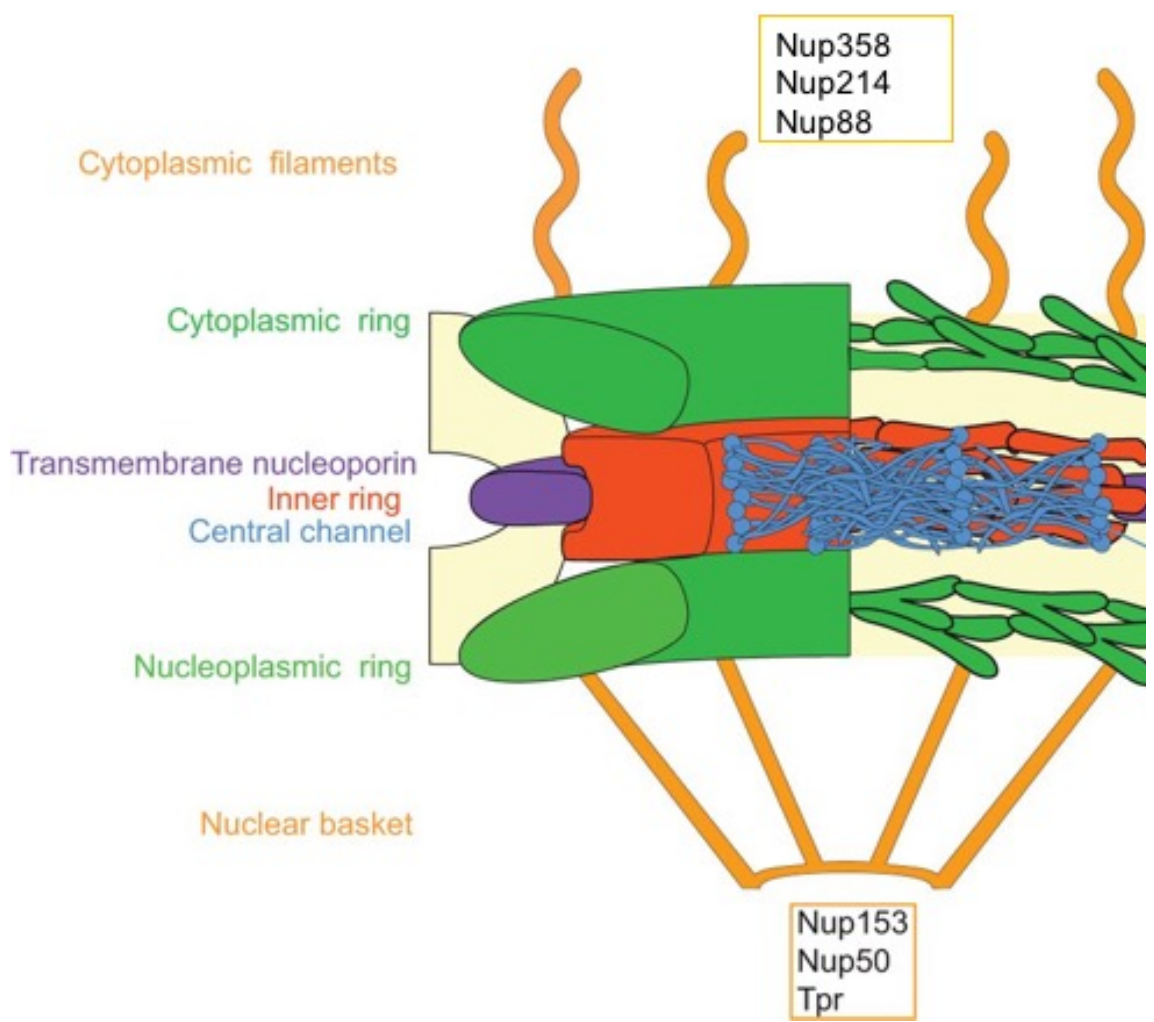

Figure 1: The arrangement of the nuclear pore complex (NPC). The NPC is made of a central channel, cytoplasmic filaments and a nuclear basket. The central channel is blocked by unstructured flexible FG-repeat domains. A top view of the NPC shows a symmetrical eight-fold arrangement (modified from Weberruss \& Antonin, 2016).

\subsubsection{The arrangement of the NPC}

Characterization of the NPC structure has been a challenge for decades, due to many reasons including their big size, unclear assembly pattern, high flexibility of some of its components and the fact that the NPC has intrinsically disordered FG-repeat domain (Ryo et al., 2017).

A characteristic feature of the NPC that deals with its complexity is the ability to organize the nucleoporins into sub-complexes (Grandi et al., 1993; Fabre \& Hurt, 1997; Rout et al., 2000; Lutzmann et al., 2002; Devos et al., 2006; Yoshida et al., 2011; Gaik et al., 2015). Several biochemical, genetic and electron microscopy studies have been conducted to understand the organization of the NPC (Fischer et al., 2015; reviewed in Beck \& Baumeister, 2016; reviewed in Lin \& Hoelz, 2019). 
The sub-complexes of the NPC are arranged into three distinctive layers according to their location within the NPC (Rout et al., 2000; Zwerger et al., 2016; reviewed in Lin \& Hoelz, 2019). The three layers are the nuclear basket, the NPC core and the cytoplasmic filaments. The backbone of the NPC is made up of a group of four transmembrane nucleoporins (Nups) known as transmembrane ring Nups (GP210, NDC1 and POM121, TMEM33), which are embedded into the nuclear envelope (Rout et al., 2000; Lin et al., 2016). The central channel is formed by a group of Nups known as channel (FG) Nups (Nup54, Nup58, Nup62 and Nup98), which are thought to interact with transport receptors via their FG repeats, and hence control the trafficking of different molecules across the NPC. The FG Nups are connected to the transmembrane Nups by adaptor Nups (Nup35, Nup93, Nup155, Nup188 and Nup205) (Rout et al., 2000; Zwerger et al., 2016; Kosinski et al., 2016; Lin et al., 2016). The Nups of the central channel are flanked at both ends by a complex of scaffold Nups known as Y-complex or the Nup107-Nup160 complex (Nup160, Nup133, Nup107, Nup96, Nup85 and Sec13), which is considered as the major building block of the NPC. The Y-complex is decorated on the cytoplasmic side by cytoplasmic filaments and on the nuclear side by a nuclear basket with an inner ring having eight filaments extending from it to unite in a distant ring (Zwerger et al., 2016; Lin \& Hoelz, 2019) (Figure 1).

\subsubsection{The cytoplasmic filaments of the NPC}

The nucleoporins of the cytoplasmic face of the NPC have been connected to several transport pathways (Middleton et al., 1984; Richardson et al., 1988; Cordes et al., 1993; Fornerod et al., 1995; Grandi et al., 1995; Bastos et al., 1997; Fornerod et al., 1997a; Delphin et al., 1997; Walther et al., 2002; Hutten et al., 2009). Additionally, these nucleoporins have been characterized biochemically and structurally for their interactions with several import and export receptors. The main characteristic proteins of the cytoplasmic side are Nup62, Nup88, Nup214 and Nup358 (Fornerod et al., 1995; Bastos et al., 1997; Shah et al., 1998; Gaik et al., 2015). Nup214 is known to be existing as a sub-complex with Nup88, while Nup358 is the largest nucleoporin of the NPC characterized by its cytoplasmic filaments projecting out of the NPC away from the nuclear envelope (Cordes et al., 1993).

- Nup214/CAN

Before Nup214/CAN was originally identified as a nucleoporin (Kraemer et al., 1994; Fornerod et al., 1995), it was first characterized as part of a genetic translocation to the DEK or SET genes, which contributes to the occurrence of acute myeloid leukemia (Garçon et al., 2005). In fact, there is still no clear correlation between the occurrence of Nup214/DEK or Nup214/SET 
translocations and the onset of leukemia. With a molecular weight of nearly $214 \mathrm{kDa}$, it is the second largest nucleoporin of the NPC (Shah et al., 1998). Nup214 is exclusively localized to the cytoplasmic side of the NPC. It is always found in complex with Nup88 (Bastos et al., 1997; Fornerod, Ohno, et al., 1997a; Bernad et al., 2006).

The overall sequence of Nup214 is not conserved between different organisms, with yeast Nup214 homolog (Nup159) having a molecular weight of around $159 \mathrm{kDa}$. Additionally, the arrangement of yeast Nup159 domains is different to that of human Nup214. Nup159 has an Nterminal $\beta$-propeller domain, followed by an FG-stretch of amino acids in the center and finally a C-terminal coiled-coil domain (Napetschnig et al., 2007). The human Nup214 sequence consists of an N-terminal $\beta$-propeller comprising the first third of the protein, a coiled-coil domain comprising the center of the structure and the FG-repeat comprising the C-terminus (Figure 2; Bernad et al., 2006; Napetschnig et al., 2007). The central region of Nup214 has been postulated for its importance in targeting of Nup214 to the NPC (Fornerod et al., 1995), while the C-terminal FG-repeat domain was shown to interact with chromosome maintenance region 1 (CRM1; discussed in more details in section 1.2.2) and contribute to CRM1 mediated cargo export (Fornerod, Ohno, et al., 1997a; Kehlenbach et al., 1998; Port et al., 2015). Several studies showed that Nup214 has a high affinity for CRM1. Additionally, the Nup214 FG-repeat region can interact with CRM1 in a RanGTP dependent manner (Roloff et al., 2013; Port et al., 2015). It was also shown that the FG-repeat region of Nup214 can stabilize an export complex consisting of CRM1, RanGTP and a cargo (Kehlenbach et al., 1998; Port et al., 2015). Human CRM1 was first identified to interact with the C-terminus FG-repeat of Nup214 in Roloff et al. (2013) and later this interaction was structurally characterized (Port et al., 2015). Additionally, a strong interaction has been characterized between XPOI (CRM1 yeast homolog) and Nup159 (Nup214 yeast homolog) (Allen et al., 2002).

Deletion of Nup214 from the genome in mice, led to early embryonic death (van Deursen et al., 1996). Additionally, the depletion of Nup214 in eukaryotic cells led to defect of RNA export (van Deursen et al., 1996), cell cycle arrest and decreased cell growth (Moroianu et al., 1995). These findings suggest that Nup214 is necessary for survival (van Deursen et al., 1996). Nup214 was shown to be necessary for recruitment of the DEAD-box helicase Ddx19 via its N-terminus (Napetschnig et al., 2007; Montpetit et al., 2011) and for export of some proteins (Hutten and Kehlenbach, 2006). The role of Nup214 in nuclear export has been extensively studied. Previous reports showed a Nup214/CRM1 dependent export of some cargos such as the nuclear factor of activated T-cells (NFAT; Kehlenbach et al., 1999). 


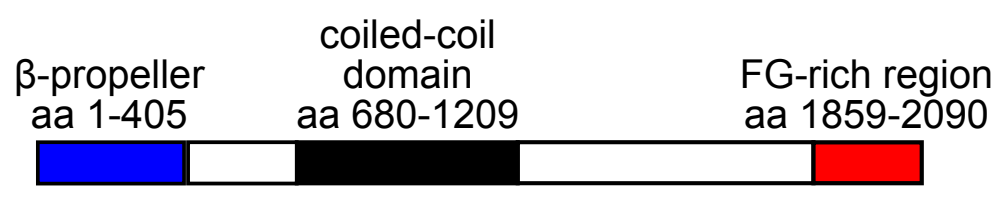

Figure 2: Domains of Nup214. Nup214 has an N-terminal ß propeller domain (shown in blue), a coiled-coil domain (shown in black) and a C-terminal FG-repeat domain (shown in red).

\section{- $\quad$ Nup88}

Nup88 is localized at the cytoplasmic side of the NPC in complex with Nup214. Nup88 was initially characterized as a binding partner of Nup214 (Bastos et al., 1997; Fornerod, et al., 1997). The Nup88-Nup214 sub-complex is localized to the cytoplasmic side of the NPC (Kraemer et al., 1994; Bastos et al., 1997). In fact, forming a stable subcomplex of Nup88Nup214 is required for the successful localization of the two nucleoporins to the cytoplasmic side of the NPC. Nup88 is known to interact with Nup214 via their coiled coil domains (Fornerod et al., 1997). Some studies pointed out that Nup88 acts as a bridge connecting Nup214 to the NPC (Roth et al., 2003).

The organization of Nup88 and Nup214 domains is similar. Both proteins have a $\beta$-propeller region at the N-terminus. However, unlike Nup88, Nup214 has an FG-repeat sequence at the C-terminus. Additionally, Nup214 has a domain known as the C-terminal extended peptide segment or CTE that regulates the functions of Nup214 through undergoing phosphorylation and de-phoshorylation events (Napetschnig et al., 2007).

In yeast, the Nup214 homolog (Nup159), Nup88 homolog (Nup82) and Nup62 homolog (Nsp1) were structurally characterized (Gaik et al., 2015). The presence of this sub-complex was found to be important in CRM1-mediated nuclear export of $60 \mathrm{~S}$ pre-ribosomal subunits in human (Bernad et al., 2006) and mRNA in yeast (Hurwitz et al., 1998). Depletion of Nup88-Nup214 sub-complex was found to cause defects in mRNA export (Gorsch et al., 1995). Nup214 and Nup88 fusion proteins have been studied for their link to tumors (Mendes and Fahrenkrog, 2019). Nup88 fusions and Nup214 fusions were found to be overexpressed in a wide range of different malignant tumors.

\section{- Nup 358/RanBP2}

Nup358/RanBP2 is the largest nucleoporin of the NPC (Wu et al., 1995; Yokoyama et al, 1995; Splinter et al., 2010). It forms cytoplasmic filaments, which are $50 \mathrm{~nm}$ long and $5 \mathrm{~nm}$ thick and acts as a docking site for several cargos and nuclear transport receptors (NTR) (Richardson et al., 1988; Delphin et al., 1997; Bernad et al., 2004). Nup358 was shown to interact with both Nup88 and Nup214 in vivo (Bernad et al., 2004). Nup358 is an essential for nucleocytoplasmic transport. It was linked to several roles in mitosis and nuclear transport. The role of Nup358 in 
the nucleocytoplasmic transport of cargos and NTR was reported in several studies. Nup358 has been linked to several transport receptors such as CRM1 (Engelsma et al., 2004; Hutten et al., 2006), importin $\alpha / \beta$ (Hutten et al., 2008; Ocwieja et al., 2011), Importin 7 (Frohnert et al., 2014) and transportin 3 (Ocwieja et al., 2011). Nup358 has a multi-domain configuration that gives it a wide spectrum of functions in various cellular mechanisms including nucleocytoplasmic transport, axon specification in developing neurons (Khalaf et al., 2019) and stabilization of microtubules (Josepha and Dasso, 2008). Additionally, several studies characterized Nup358 to be involved in various other processes such as regulating the polarity of the cell as well as its role in controlling the microtubule dynamics (Joseph and Dasso, 2008; Murawala et al., 2009). The effect of Nup358 depletion was variable among different studies. Bernad et al. (2004) showed that Nup358 depletion resulted in loss of Nup214, while Hutten et al. (2006) showed that Nup358 depletion does not affect Nup214. 


\subsection{The nucleocytoplasmic transport}

The central channels of the NPC are formed by nucleoporins harboring the FG repeat domains. These FG-repeats act as a permeability barrier that prevents free diffusion of large molecules across the NE (Lim et al., 2006). As a general rule, the FG repeat permeability barrier of the NPC allows molecules smaller than $40 \mathrm{kDa}$ to diffuse through (Terry \& Wente, 2009) and molecules larger than $40 \mathrm{kDa}$ to be actively transported via a nuclear transport receptors. The transported molecules need specific transport receptors that have the ability to interact with the NPC barrier.

Active transportation occurs by a direct or indirect (via an adaptor protein) interaction between the nuclear transport receptor and the cargo. The nuclear transport receptor shuttles between the cytoplasm and the nucleus by transiently interacting with the FG repeat permeability barrier of the NPC central channel to reach the cytoplasmic or the nuclear sides of the NPC, where the cargo is released (Bayliss et al., 1999; Chook \& Blobel, 1999; Gorlich \& Kutay, 1999; Bayliss et al., 2000).

The directionality of the transport process is dependent on a gradient of RanGDP/RanGTP across the NPC. The process is controlled by a group of proteins and factors namely: Ran, RanBP1, GTPase activating protein (RanGAP1) and a guanine nucleotide exchange factor (RCC1) (discussed in more details in section 1.2.1) (Izaurralde et al., 1997; Nachury \& Weis, 1999; Fried \& Kutay, 2003).

Many proteins and RNAs depend on a family of transport receptors known as karyopherins for transport across the NE (Cook et al., 2007). For transport of the cargo, it has to be recognized by the transport receptor. This recognition occurs through short sequences on the cargo. The sequence can either be a nuclear localization signal (NLS) that facilitates the transport of the cargo to the nucleus, or a nuclear export signal (NES) that initiates its transport to the cytoplasm (discussed in more details in section 1.2.3). On their own, the karyopherins can diffuse across the NE and get recycled several times for transport. When the right conditions are available, a karyopherin will bind a cargo and facilitate its transport.

Several studies that were dedicated to the understanding of transport across the NPC provided a model of nucleocytoplasmic transport (Rout et al., 2003; Lin et al., 2016). The three main components of this model are the transport receptors, the Ran machinery and the targeting signal. 


\subsubsection{Ran dependent nucleocytoplasmic transport}

Ran is classified as a GTPase of the Ras superfamily (Gorlich \& Kutay, 1999; Fried \& Kutay, 2003). The nucleotide bound state of Ran shifts between GDP and a GTP states thanks to two factors, RanGAP1 and RCC1.

GTPase activating protein (also known as RanGAP1) is exclusive to the cytoplasmic side of the NPC where RanBP1 and Nup358 are present (Hopper et al., 1990; Melchior et al., 1995; Richards et al., 1996). The GTPase activity of Ran is amplified 10,000 folds by RanGAP1 and 100,000 folds when RanBP1 is additionally present (Bischoff et al., 1995). By contrast, the guanine exchange factor (also known as RCC1) is exclusive to the nucleus due to its dynamic association with the histones H2A and H2B (Ohtsubo et al., 1989; Nemergut et al., 2001). As a result of the localization of RCC1 in the nucleus and RanGAP1 in the cytoplasm, Ran retains a GTP bound state in the nucleus and a GDP bound state in the cytoplasm (Figure 3). This change in localization leads to a gradient of RanGTP across the NPC which ensures the directionality of transport. The translocation of RanGDP to the nucleus is mediated by NTF2 which has a high affinity for Ran in GDP state. NTF2-RanGDP complex is formed in the cytoplasm prior to translocation to the nucleus. The release of Ran from NTF2 in the nucleus is initiated by the RCC1-mediated nucleotide exchange that generates RanGTP. NTF2 has a low affinity for RanGTP and this leads to disassembly of the NTF2-Ran complex (Ribbeck, 1998) (Figure 3).

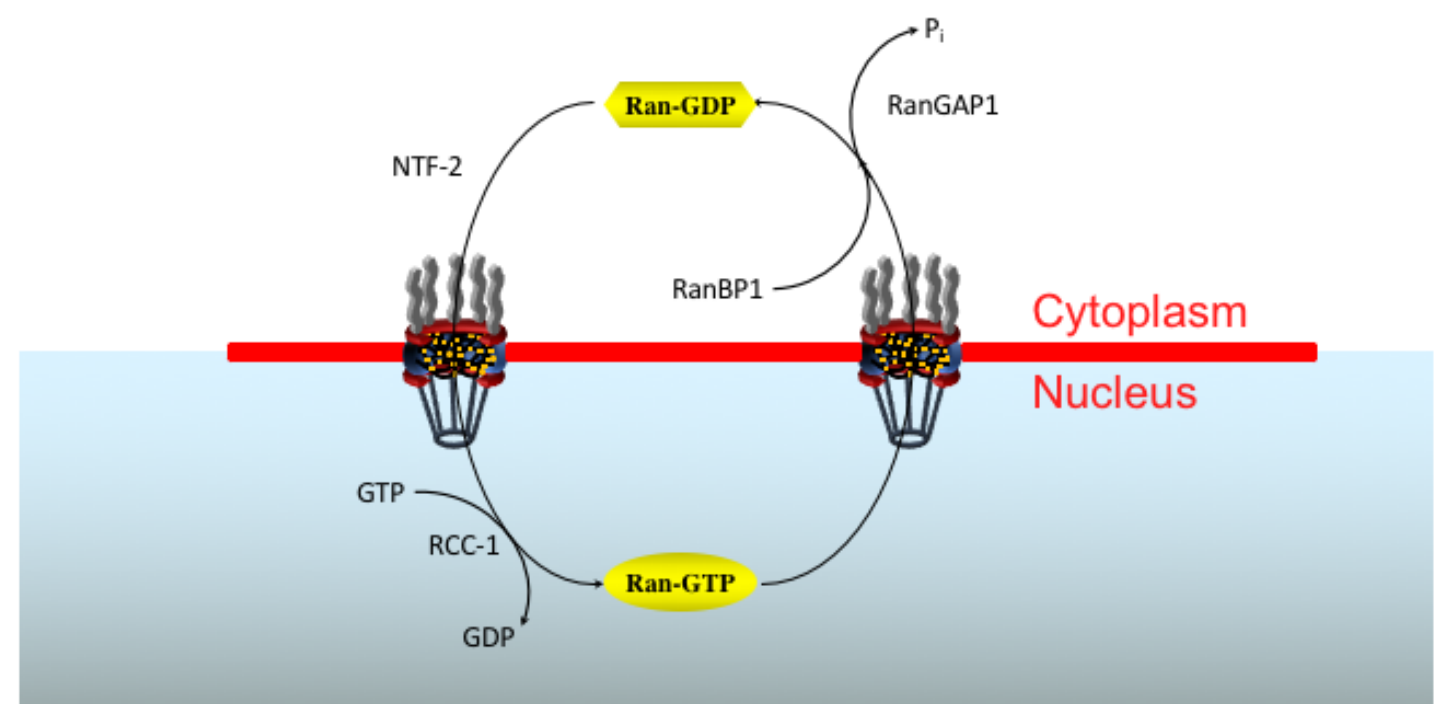

Figure 3: Schematic representation of the Ran cycle. Ran is a necessary component of nucleocytoplasmic transport. Ran changes its state according to its location within the cell by the help of RanGAP1 and RCC-1.

The nucleotide bound state of Ran determines its interaction behavior. RanGTP has a high affinity for transport receptors. Once an import complex reaches the nucleus, the RanGTP binds to the complex and induces a conformational change which releases the import substrate. By 
contrast GTP hydrolysis by RanGAP1 and Nup358 at the cytoplasmic side decreases the affinity of Ran for the import receptors and thus allows an import cargo to bind the import receptor in the cytoplasm. In addition, GTP hydrolysis disassembles the export complexes reaching the cytoplasmic side of the NPC (Figure 4).

The directionality of nucleocytoplasmic transport is controlled by a group of key players including the nuclear import/export receptors, the regulatory components of the Ran GTPase cycle and the GTPase Ran (Görlich et al., 1996; Schlenstedt \& Solsbacher, 1999). The disassembly machinery of trimeric export complexes is made up of RanBP1 and RanGAP associated with Nup358. RanGTP is shielded from RanGAP by transport receptors. Once RanBP1 binds RanGTP, the latter becomes exposed to RanGAP and finally the export complex is dissociated (Matunis et al., 2016; Mahajan et al., 1997; Künzler \& Hurt, 2001; Fried \& Kutay, 2003). The interaction between RanGAP1 and Nup358 is initiated by SUMOylation of the RanGAP1 C-terminal domain with the ubiquitin-related modifier SUMO1 (Matunis et al., 2016; Mahajan et al., 1997) in the presence of the SUMO E2 conjugating enzyme Ubc9 (Saitoh et al., 1997; Ritterhoff et al., 2016). It is suggested that RanGAP1 interaction with SUMO1/Ubc9 functions is the preferable disassembly machine for CRM11-mediated export of cargoes (Ritterhoff et al., 2016).

Nup358 was characterized as a SUMO E3 ligases that assists the SUMO-modification of RanGAP1 (Pichler \& Melchior, 2002; Werner et al., 2012; Sakin et al., 2015). Localization studies showed that Ran GTPase-activating protein RanGAP1 accumulated and formed a stable complex with Nup358 at the cytoplasmic periphery of the NPC (Melchior et al., 1995; Matunis et al., 2016; Mahajan et al., 1997, Hutten et al., 2008; Werner et al., 2012). Early studies showed that an ubiquitin-related protein known as SUMO-1 modifies RanGAP1 and targets it to Nup358 at the NPC. The co-existence of Nup358 and RanGAP1 at the cytoplasmic side of the NPC was found important for protein import (Mahajan et al., 1997). In addition to its role in the targeting of RanGAP1 to Nup358 and protein import, the SUMOylation of RanGAP1 was linked to regulating the direction of the transport of some cargoes across the NPC (Sakin et al., 2015). The functional implication of RanGAP1-RanBP2 was well characterized in mitosis, where RanGAP1-RanBP2 complex re-localized to the kinetochores and spindle fibers in mitosis (Joseph et al., 2002; Joseph et al., 2004; Gilistro et al., 2017). Depletion of Nup358 in mitotic cells compromised the kinetochore structure and function, resulted in failure of mitosis and formation of multinucleated cells (Salina et al., 2003; Joseph et al. 2004; Gilistro et al., 2017). The exportin CRM1 is an important factor in the recruitment of the RanGAP1-RanBP2 complex to the kinetochores (Arnaoutov et al., 2005). 


\subsubsection{Transport receptors (karyopherins)}

Previous studies have established that a certain class of proteins as NTF2-like proteins and Importin $\beta$ possess the capability to interact with the FG-repeat Nups to cross the NPC. These studies also demonstrated that such transport receptors have a high affinity for their cargoes and hence are responsible for translocating them through the NPC. The family of transport receptors (also known as karyopherins) can be subdivided into importins or exportins, depending on whether they promote import of the cargo into the nucleus (importins) or export of the cargo out of the nucleus (exportins) (Iovine et al., 1995, Fornerod et al., 1997a; Görlich et al., 1997; Arts et al., 1998; Ribbeck, 1998; Shah et al., 1998; Allen et al., 2001; Strawn et al., 2001; Bayliss et al., 2002; Mosammaparast \& Pemberton, 2004). Some karyopherins such as Importin 13, were described to function as both an import receptor and export receptor (Mingot et al., 2001)

An important component of nucleocytoplasmic transport is the superfamily of karyopherin transport receptors (reviewed in Moroianu, 1998; Yuh \& Blobel, 2001; Ström \& Weis, 2001). For export to take place, a class of karyopherins known as exportins are needed (Kutay \& Güttinger, 2005; Pemberton \& Paschal, 2005). An export complex is formed by binding of the exportin to the cargo NES and RanGTP. Once the complex is formed it will be carried across the NE to the cytoplasm. When the export complex reaches the cytoplasm, it docks at Nup358 where RanGAP1 in combination with RanBP1 initiate the hydrolysis of RanGTP to RanGDP. This leads to the release of the export cargo and the exportin into the cytoplasm (Kehlenbach et al., 1999). By contrast, a class of karyopherins known as importins is needed for the import of the cargo (Goldfarb et al., 2004; Pouton et al., 2007).

\section{- The nuclear export receptor chromosome region maintenance 1 (CRM1)}

The chromosome region maintenance 1 protein (CRM1), also known as exportin-1 or XPO1, is one of the most studied karyopherins (Neville et al., 1997; Ossareh-Nazari et al., 1997; Stade et al., 1997; Kehlenbach et al., 1998; Thakar et al., 2013; Kırlı et al., 2015; Fu et al., 2018). CRM1 is an important receptor that has a wide spectrum of essential cargoes (Xu et al., 2015; $\mathrm{Fu}$ et al., 2018). Since its discovery 23 years ago, the cargos of CRM1 are continuously increasing with more than 200 experimentally proven cargos already recorded in databases as validNESs (Fu et al., 2013) and NESdb (Xu et al., 2012) and over 1000 putative cargos identified in different proteomic studies (Fu et al., 2013; Kırlı et al., 2015; Xu et al., 2015). CRM1 was first identified in Schizosaccharomyces pombe strains (Adachi \& Yanagida, 1989). CRM1 interactions with PKA inhibitor (PKI) has been well characterized in the context of PKI function in phosphorylation of proteins (Wen et al., 1995). Additionally, CRM1 export 
complexes have been formed and characterized including CRM1-RanGTP-SPN1 (Dong et al, 2009; Monecke et al., 2009), CRM1-RanGTP-PKI (Wen et al., 1995) and CRM1-RanGTPHIV-1 Rev (Fischer et al., 1995). However, the importance of CRM1 is not limited to that. In fact, CRM1 is involved in the infection cycle of many viruses. Viral proteins of human immunodeficiency virus (HIV) and parvovirus minute virus of mice (MVMp) have been characterized for having an NES that interacts with CRM1 (Eichwald et al., 2002). CRM1RanGTP-HIV-1 Rev ( Fischer et al., 1995; Askjaer et al., 1998) was studied for its role in the export of HIV viral RNA to the cytoplasm ( Fischer et al., 1995; Neville et al., 1997).

The importance of CRM1 has shed light on the possibility for becoming a clinical target. One of the early studied inhibitors of CRM1 is leptomycin B (LMB). LMB is a secondary metabolite that has been isolated from Streptomyces spp. (Nishi et al., 1994) and is the most studied inhibitor of CRM1 (Hamamoto et al., 1983; Adachi \& Yanagida, 1989). It acts by modification of the cysteine residue C528 of human CRM1, thus obstructing the binding of an NES and inhibiting protein export (Kudo et al., 1998). Many inhibitors have been later identified and tested in clinical trial as possible antiviral and anti-cancer candidates. Some examples for these inhibitors include KPT-350 and KPT-335 (also known as Verdinexor) and KPT-330 (also known as Selinexor) (Fu et al., 2018). Several studies (Lapalombella et al., 2012; Ranganathan et al., 2012; Etchin et al., 2013)) focused on the importance of CRM1 as a possible target in treatment of pancreatic cancer, breast cancer and leukemia (Fornerod et al., 1997b; Kojima et al., 2013; Port et al., 2016; Sun et al., 2016; Muqbil et al., 2018).

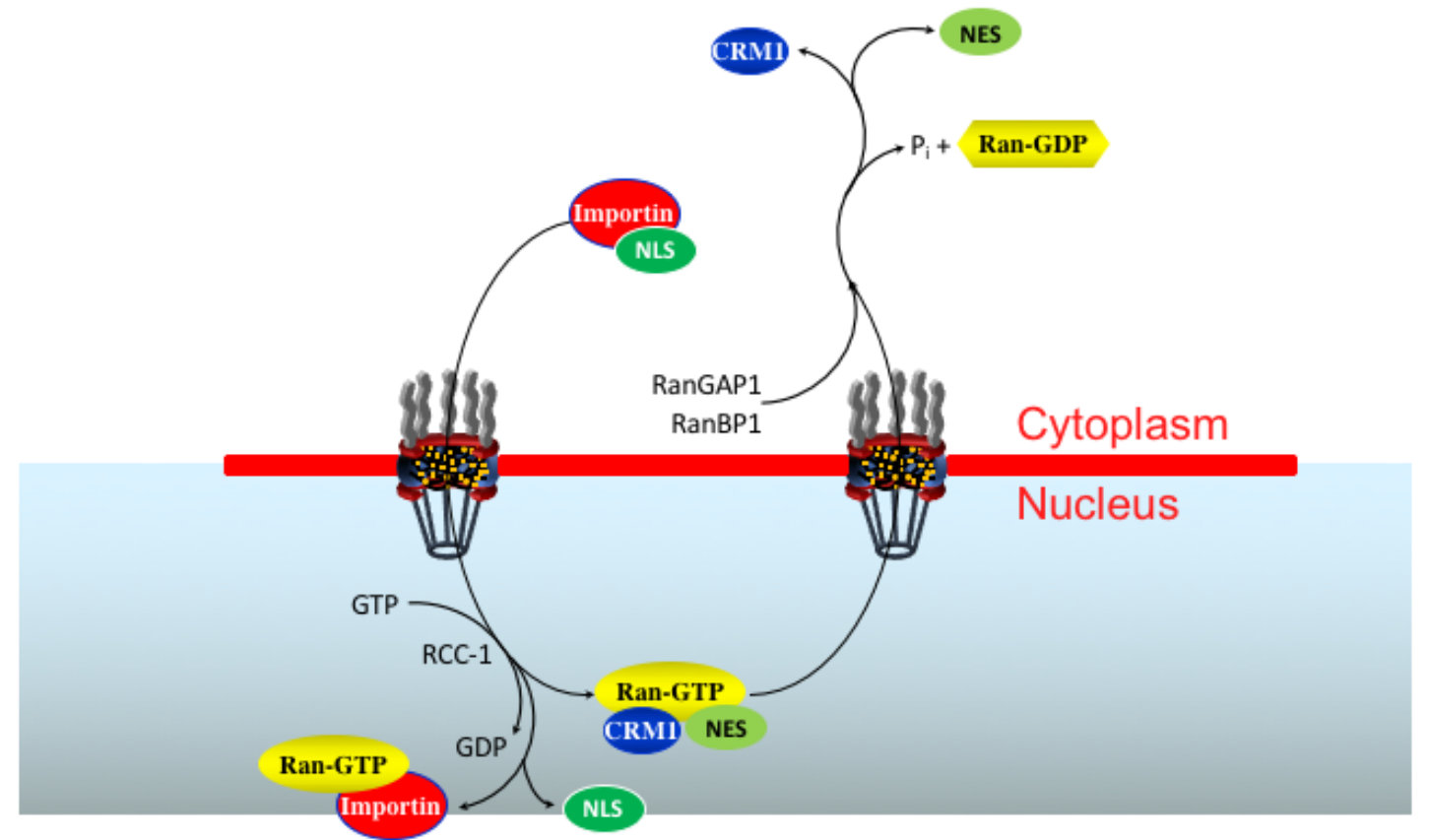

Figure 4: Nucleocytoplasmic transport. Nucleocytoplasmic transport is an active process mediated by importins or exportins. Either an NLS-containing protein is imported via an importin, or an NES-containing protein is exported by an exportin in presence of RanGTP. 


\subsubsection{Nuclear localization/Nuclear export signals}

The diversity of transport pathways within the cell can be reflected, not only by the diversity of the transport receptors, but also by the diversity in the conformation, sequence and spacing of the targeting signals that bind to these receptors. This diversity leaves a big space for a wide array of different transport pathways that the cell can use to transport its molecules.

Nuclear localization signal (NLS) can occur as a simple NLSs or a bipartite NLSs. Simple NLSs- as in case of SV40 large T antigen- are made up of a short sequence enclosing a cluster of basic amino acids which are usually followed by a proline residue or an acidic amino acid. By contrast, bipartite NLSs- as in case of nucleoplasmin- are made up of two independent short sequences containing two independent clusters of basic amino acids which are separated from each other by a flexible sequence. The main transport receptor for import of nuclear export signal (NLS) containing proteins is the Importin $\alpha /$ Importin $\beta$ heterodimer, which translocate import complexes to the nucleus (Gorlich et al., 1995).

NES are characteristic for CRM1 export cargos. Several NES-containing proteins have been well established and characterized including protein kinase inhibitor protein (PKI, Wen et al., 1995), HIV1-Rev NES (U. Fischer et al., 1995; Askjaer et al., 1998) and Snurportin-1 (SPN1) which was described to have a very high affinity for CRM1 (Paraskeva et al., 1999). Early studies referred to the NESs as leucine rich repeats made of 8-15 residue-long cluster which contains four key hydrophobic residues ( $\Phi$; Fischer et al, 1995; Fornerod et al., 1997a; Neville et al., 1997). The partitioning of these residues followed the consensus $\Phi 1-(\mathrm{X})_{2-3}-\Phi 2-(\mathrm{X})_{2-3^{-}}$ $\Phi 3-X-\Phi 4$, where $\Phi$ is a hydrophobic amino acid (L, I, V, F, M) and X can be any amino acid (Kutay \& Güttinger, 2005). In recent years, the sequence and the partitioning of the NES consensus was refined. Crystallization of SPN1-RanGTP-CRM1 (Dong et al, 2009; Monecke et al, 2009) revealed that SPN1 binds CRM1 through five, rather than four, hydrophobic $(\Phi)$ residues. According to (Güttler et al., 2010), screening of several classic NESs has revealed that indeed an extra hydrophobic residue $(\Phi 0)$ binds to the NES-docking cleft of CRM1. It was also found that CRM1 possesses five hydrophobic pockets that can bind the five hydrophobic residues of an NES. As a result of these studies, the current consensus would be as follows; Ф0X-Ф1-(X) 2-3-Ф2-(X) 2-3-Ф3-X-Ф4 (Güttler \& Görlich, 2011). The NES have been classified into different classes according to the position of the hydrophobic residues as well as the space between them (Fung et al., 2015). The most recent definition of an NES motif is 'it is a short cluster of hydrophobic residues which (a) contains four to five hydrophobic residues (Ф0- Ф4), (b) $\Phi$ can be any of the hydrophobic amino acids: L, I, V, F, M and (c) is bound to the CRM1 hydrophobic pockets P0-P4' (Fu et al., 2018; Lee et al., 2019). 


\subsubsection{Nucleocytoplasmic transport models}

There are several theories about how the transport receptors interact with the NPC barrier. Some studies suggested that the FG-repeats forming the transport barrier can interact with hydrophobic regions of the transport receptors (Lin et al., 2016). It is also known that several transmembrane and soluble proteins have been identified in separate proteomic analysis studies for the presence of FG-repeats in their structures. In fact, it was shown that the FG repeats of these proteins enabled them to act as their own transport receptors (Kerr \& Schirmer, 2011). It is thought that the FG-repeats on the NTR or the cargo transiently interacts with the FG-repeats forming the central NPC channel and thus translocate across the NE.

- The 'Virtual gating model' (Rout et al., 2003) suggests that the NPC acts as a catalyst that reduces the energy required for the translocation of molecules. The term 'virtual gating' was used to describe this model because the selectivity is independent on any mechanical barriers. Virtual gating model describes the translocation of large molecules through the NPC to be restricted because they need to pass through a narrow FG-repeat-crowded NPC tunnel and therefore they have low entropy. By contrast, transport receptors can interact with the FGrepeats and overcome this entropic barrier. Cargoes bound to transport receptors have high entropy and can pass though the central channel of the NPC (Rout et al., 2003).

- The 'Brownian affinity model', suggests that transport across the NPC depends on the concentration of the nuclear transport receptor (NTR)-cargo complex existing one side of the NPC. For transport to happen, the NTR-cargo complex forms at one side of the NPC and accumulate at the entrance of the central channel. This increases the residence time of the complex at the entrance and thus increases their probability of entering the central channel. The NTR-complex then moves into the channel down its concentration gradient following Brownian motion. According to this model, the NTR-cargo complex finally docks to the FGnucleoporins at the cytoplasmic or nuclear side of the NPC to release its cargo. The model also proposed that import complexes do not need to dock to the cytoplasmic side of the NPC to get imported. However, the model failed to explain how large assemblies such as ribosomes can pass through the NPC (Rout et al., 2000; Walther et al., 2002; Rout et al., 2003).

- The 'selective phase model'(Ribbeck \& Görlich, 2001) proposes that the unstructured intrinsically disordered FG repeats forming the central channel of the NPC interact with each other to form a sieve-like barrier that gets disrupted by NTR translocating across the NPC. The model suggests that molecules are transported across the NPC in a selective manner. As a result, inert molecules, which are not bound to a NTR are not allowed a passage though the NPC. The model is based on the tendency of the central channel FG repeats to form in-vitro macroscopic 
hydrogels (Ader et al., 2010). The transport receptors, whether alone or in complex with a cargo, can interact with the phenylalanine-glycine meshwork and permeabilize the mesh. A reconstitution of recombinant FG domains experimentally, showed that -under certain conditions- they could form a hydrogel-like phase through which NTR-cargo complexes can pass (Frey et al., 2006). A very recent work showed that a liquid to liquid phase separation of FG-Nups -which mimics the NPC permeability barrier- allowed the passage of functional import complexes into the liquid phase but did not allow the passage of proteins that are not bound to an import receptor. This study suggested that FG-repeats in the central channel can alternate between a liquid phase and a solid phase (Dormann, 2020).

- The 'reduction of dimensionality (ROD) model' (Terry \& Wente, 2009; Peters, 2009), suggests that the FG-repeats of the central channel interact together to form a single coherent layer on the central channel of the NPC. However, unlike the 'selective phase model', the FGrepeats do not fully close the channel but rather leave an opening of $10 \mathrm{~nm}$ wide to allow the passage of small molecules. The model suggests that NTR alone or in complex interact transiently with the surface of the FG-repeat layer to pass the NPC. Such arrangement is assumed to provide a certain degree of flexibility for transport of very large molecules such as ribosomal complexes. 


\subsection{Assembly and disassembly of the NPC}

The NPC is one of the largest protein complexes in cells and the average half-life of its proteins is few days (Cambridge et al., 2011; Savas et al., 2012). In order for the NPC to maintain itself, it has to clear its damaged components by a slow but predetermined turnover of its individual subcomplexes (Toyama et al., 2014). A cycle of assembly and disassembly of the NPC occurs in dividing cells depending on the stage of the cell cycle (reviewed in Kabachinski \& Schwartz, 2015). Data from several studies suggested a model for the assembly of the NPC during interphase and at post-mitotic exit (Ryan et al., 2003; D'Angelo et al., 2006; Antonin et al., 2008; Flemming et al., 2009; Onischenko et al., 2009; Dultz \& Ellenberg, 2010; reviewed in Weberruss \& Antonin, 2016; reviewed in Otsuka \& Ellenberg, 2018; Hampoelz et al., 2019). The assembly of the NPC occurs via two distinct pathways during the cell cycle, first at postmitotic exit and second at interphase (Flemming et al., 2009; Onischenko et al., 2009; Doucet et al., 2010; Hampoelz et al., 2016).

\section{- Post-mitotic assembly of the NPC}

In eukaryotic cells, the NPCs are phosphorylated by mitotic kinases and disassembled during mitosis into soluble sub-complexes (Laurell et al., 2011). Some studies (Orjalo et al., 2006; Zuccolo et al., 2007) pointed out the role of the released sub-complexes in the progress of mitosis. Y-complex was found to be important for the assembly of the mitotic spindles. In addition, the Nup88-Nup214 subcomplex was localized on the spindles during mitosis and the depletion of Nup88 resulted in a multipolar spindle phenotype and aneuploidy (Hashizume et al., 2010).

Post-mitotic assembly refers to a stage where NPCs are rapidly assembled from pre-existing soluble subcomplexes with re-formation of the NE at the end of open mitosis (Dultz et al., 2008). It occurs during the reformation of the nuclear envelope. During this time, thousands of NPCs are regenerated and the transport competence of the NE is restored within few minutes (Dultz et al., 2008).

At the onset of post-mitotic assembly, the Y-complex is targeted to the chromatin by the help of small-DNA-binding motifs present in ELYS (Franz et al., 2007; Rasala et al., 2008; Doucet et al., 2010). After ELYS is localized at the chromatin, the Y-complex assembly continues and the Y-complex interacts with Ndc1 and Pom121 (Dultz et al., 2008; Rasala et al., 2008). The recruitment of Nups to the chromatin is regulated by Ran and importin $\beta$ (Harel et al., 2003; Walther et al., 2003). Next, Nup93 subcomplex members are recruited (Dultz et al., 2008) to help in stabilization of the NPC (Eisenhardt et al., 2014). Finally, the FG-Nups such as Nup214 are recruited and nuclear pores become functional (Dultz et al., 2008). The chronological 
control of the events occurring at post-mitotic stage occurs via cell-cycle-dependent phosphatases and kinases (Weberruss \& Antonin, 2016; Ungricht \& Kutay, 2017).

It is important to consider the variances in the state of the nuclear envelope between organisms during mitosis. S. cerevisiae has a 'closed' mitosis, where the NE remains intact throughout mitosis because the microtubule organizing center (MTOC) is embedded in the NE. Other organisms, where the MTOC is cytoplasmic, have an 'open' mitosis which requires the breakdown of the NE so that chromosome segregation occurs correctly. Some studies (De Souza et al., 2004, De Souza \& Osmani, 2007) described 'semi-closed' mitosis where the NPC is partially disassembled. These variations affect the NPC assembly/disassembly and might have resulted in different interphase and post-mitotic assembly pathways (D'Angelo and Hetzer, 2008; Doucet et al., 2010).

\section{- Interphase assembly of the NPC}

In interphase, new NPCs are formed and assembled de-novo into an intact NE (D'Angelo et al., 2006; Dultz and Ellenberg, 2010). Interphase assembly is the only mode of NPC assembly for organisms with a closed mitosis (Winey et al., 1997). By contrast to post-mitotic assembly, interphase is a slower process (Doucet et al., 2010). At the onset of interphase, the surface area of the nuclear envelope increases and the NPCs number doubles to prepare the cell for division or in response to changes in the metabolic activity of the cell (Doucet et al., 2010; Dultz \& Ellenberg, 2010). Similar to post-mitotic assembly, interphase NPC assembly requires the presence of the Ran system and importins (D’Angelo et al., 2006). In fact, it was previously shown that an NLS sequence in Pom121 was required to direct the protein to the inner nuclear envelope (Yavuz et al., 2010; Funakoshi et al., 2011). In addition, Nup153 has to be imported into the nucleus to bind the inner nuclear membrane and recruit the Y-complex to the nuclear side of the NPC (Vollmer et al., 2015). It was also found that binding of transportins to Nup153 NLS masked the membrane-binding motif of Nup153 and thus prevented its binding to the cytoplasmic side of the NPC. Once Nup153 is imported into the nucleus, Nup153-importin complex is disassembled and Nup153 is released to function in NPC assembly (Vollmer et al., 2012; Vollmer et al., 2015). Indeed, Nup153 was shown to be vital for interphase NPC assembly (Doucet et al., 2010; Vollmer et al., 2015).

Different mechanisms of NPC assembly at interphase were proposed. The first mechanism suggests that the outer and the inner NE separate to allow for a pre-formed pore to gets inserted. Then the endoplasmic reticulum (ER) cisternae reseals the NE. This mechanism was debated because the NE discontinuity would interrupt the separation between the nucleus and the cytoplasm. However, since these events occur locally, it is thought that other NPCs could 
compensate for the temporal NE discontinuity and that the overall transport capacity across the NE is not affected. In fact, some studies developed models showing that NPCs are formed away from the nuclear compartment (Hampoelz et al., 2016; Hampoelz et al., 2019). These models suggest that the endoplasmic reticulum has stacked membranes sheets known as annulate lamellae (AL) that harbor the formation of NPCs out of the nuclear compartment during oogenesis. The AL act as an NPC storage pool which provides the newly formed nuclei with further membranes and NPCs (Hampoelz et al., 2016). Furthermore, cytoplasmic Nups (Nup88 and Nup214) in addition to members of the nuclear basket (Tpr and Nup153) were absent in the AL, but highly present in the cytoplasm (Onischenko et al., 2004; Hampoelz et al., 2016). Additionally, it was suggested that the AL-NPCs are first inserted into the NE and then complexes of the cytoplasmic and nuclear sides of the NPC get recruited in late stages of the assembly process. The second mechanism suggests that the inner and outer nuclear membrane converge and fuse to form a hole into which the NPC can be assembled without compromising the diffusion barrier. However, the sequence of events and the factors mediating the proximity and fusion of the inner and outer membranes are currently unknown (reviewed in Antonin et al., 2008; Rothballer \& Kutay, 2013). As a result, transmembrane proteins residing on one or both membranes of the NE are thought to be important for linking the inner and outer membranes of the NE. Live-cell imaging combined with high-resolution electron tomography was recently used to investigate the assembly process. One study (Otsuka et al., 2016) described a 'dome-shaped invagination which forms at the inner nuclear membranes and then grows in depth and size until it fuses with the outer nuclear membrane. To form this dome-shaped invagination, the nuclear membrane has to be deformed by insertion of amphipathic helixes or via membrane binding protein which has a scaffolding function. Several Nups have been linked to deforming the nuclear membrane including the members of the Y-complex, which were found to have a scaffolding function (Devos et al., 2004; Mans et al., 2004; Brohawn et al., 2008). Nup53 was found to be critical for NPC assembly at interphase due to the presence of membrane-binding and membrane-bending helix which introduces a deformity in the nuclear membrane (Vollmer et al., 2012). Additionally, Nup133 and Nup153 have an amphipathic helix which was shown to be necessary for interphase assembly of the NPC (Doucet et al., 2010; Vollmer et al., 2015). Live-imaging kinetics studies showed that at early stages of interphase, the members of the Nup93 subcomplex are recruited to the transmembrane Nups (Flemming et al., 2009; Makio et al., 2009; Onischenko et al., 2009). This is followed by the recruitment of the Y-complex which is dependent on importin $\beta$ and the Ran cycle (Ryan \& Wente, 2002; Ryan et al., 2003; D'Angelo et al., 2006; Ryan et al., 2007; Dultz and Ellenberg, 2010). The last 
stage of assembly during interphase is the recruitment of the FG-Nups such as Nup214. FGNups localize at the NPC to generate the transport barrier of the NPC. 


\subsection{Aim of work}

Understanding the interaction between Nup214 and CRM1 can give an insight into several nucleocytoplasmic transport events across the NPC. Available data characterized the interaction between CRM1 and FG-repeat region of Nup214. This interaction is involved in export of certain CRM1 cargos.

A scan through the sequence of Nup214 by a previous PhD student in our lab, Sarah Port, has revealed a putative NES sequence. Nup214 is one of three proteins localizing to the cytoplasmic side of the NPC. A nuclear export signal (NES) was not reported in Nup214 or any other protein at the cytoplasmic side of the NPC. The putative NES is found in the central coiled-coil region of Nup214, a region that was previously reported to be important for targeting of Nup214 to the NPC and for binding to Nup88 (Fornerod et al., 1995).

This study aims at investigating the interaction of $\mathrm{NES}_{\mathrm{Nup} 214}$ with CRM1 and the effect of this interaction on the localization of Nup88. Additionally, the study investigates the role of $\mathrm{NES}_{\mathrm{Nup} 214}$ sequence in targeting and incorporation of Nup214 at the NPC in a CRM1- and possibly Nup358- dependent manner. 


\section{Materials and Methods}

\subsection{Materials}

\subsubsection{Equipment}

Table 1: Equipment

\begin{tabular}{|c|c|}
\hline Equipment & Supplier \\
\hline Agarose gel documentation GelSTICK touch & INTAS Science \\
\hline Agarose gel running chamber & UMG \\
\hline ÄKTA column HiLoad 26/10 desalting & GE Healthcare \\
\hline ÄKTA column HiLoad 26/60 Superdex 200 prep grade & GE Healthcare \\
\hline ÄKTA column HiLoad 26/60 Superdex 75 prep grade & GE Healthcare \\
\hline ÄKTA column His Trap FF 5ml & GE Healthcare \\
\hline ÄKTA column MonoQ & GE Healthcare \\
\hline ÄKTA column Superdex 200 10/300 GL & GE Healthcare \\
\hline ÄKTA purifier & Amersham Biosciences \\
\hline Autoclave sterilizer DX-200 & Systec \\
\hline BioPhotometer & Eppendorf \\
\hline CASY 1 & Schärfe System \\
\hline Cell culture hood Herasafe ${ }^{\mathrm{TM}} \mathrm{KS}$ & ThermoScientific \\
\hline Cell culture incubator Cytoperm 2 & Heraeus Instruments \\
\hline Cell culture incubatorHeracell ${ }^{\mathrm{TM}} 150 \mathrm{i}$ & Thermoscientific \\
\hline Centrifuge $5415 \mathrm{R}$ & Eppendorf \\
\hline Centrifuge 5424 & Eppendorf \\
\hline Centrifuge Allegra ${ }^{\circledR}$ X 22 with rotor SX4250 & Beckman Coulter \\
\hline Centrifuge Allegra $\AA$ X-15R with rotor SX475 & Beckman Coulter \\
\hline Centrifuge Avanti ${ }^{\mathrm{TM}} \mathrm{J}-30 \mathrm{I}$ with rotor JA30.50Ti & Beckman Coulter \\
\hline Centrifuge J6-MI with rotor JS 4.2 & Beckman Coulter \\
\hline Centrifuge Optima MAX-XP with rotor TLA120.1 & Beckman Coulter \\
\hline Centrifuge Sigma & Sigma \\
\hline CLARIOstar Plus plate reader & BMG labtech \\
\hline Confocal microscope LSM 510 meta & Zeiss \\
\hline Dual Gel Caster for Mini Vertical Units & Hoefer \\
\hline Electrophoresis Power Supply EPS 301 & Amersham Bioscience \\
\hline EmulsiFlex-C3 & Avestin \\
\hline Fluorescence microscope Axioskop 2 & Zeiss \\
\hline FluoroMax 4 flourometer & Horiba \\
\hline Gel documentation LAS-3000 & Fujifilm \\
\hline Hera freeze $-80^{\circ} \mathrm{C}$ & Thermo Electron Corporation \\
\hline Incubator Heraeus function line & Heraeus \\
\hline Incubator Shaker INNOVA 4430 & New Brunswick Scientific \\
\hline Micro centrifuge model IR 220 VAC & Roth \\
\hline Milli-Q® & Millipore \\
\hline Mini Trans-Blot ${ }^{\circledR}$ Cell & Bio-Rad \\
\hline Odyssey ${ }^{\circledR}$ CLx Imaging System & LI-COR \\
\hline Odyssey ${ }^{\circledR}$ Sa Infrared Imaging System & LI-COR \\
\hline Olympus CK40 Culture Microscope & Olympus \\
\hline Plate reader BioTek Synergy HT & BioTek \\
\hline SE250 Mighty Small II Mini Vertical Electrophoresis Unit & Hoefer \\
\hline Spectrophotometer NanoDrop 2000c & ThermoScientific \\
\hline Thermocycler FlexCycler2 & Analytik Jena AG \\
\hline Thermocycler PTC-200 DNA Engine & MJ Research \\
\hline Thermocycler Tprofessional & Biometra \\
\hline Thermomixer comfort & Eppendorf \\
\hline Thermomixer compact & Eppendorf \\
\hline UV sterilizer & Biometra \\
\hline UV transilluminator & Uvitec \\
\hline Vortexer MS2 Minishaker & $\mathrm{KA}$ \\
\hline
\end{tabular}


VortexGenie2

Water Bath Model 1003

XCell SureLock ${ }^{\circledR}$ Mini-Cell
Suedlabor

GFL

Life technologies

\subsubsection{Software}

Table 2: Software

\begin{tabular}{ll}
\hline \hline Software & Supplier \\
\hline \hline AxioVision (LE) Rel. 4.8.1 & Zeiss \\
Cell profiler 3.1.9 & Open Source \\
Centrifuge 5415R & Eppendorf \\
FluorEssence & Horiba \\
Gene 5 plate reader software & BioTek \\
GraphPad Prism 5 & GraphPad Software Inc. \\
GraphPad Prism 8 & GraphPad Software Inc. \\
Image Reader LAS-3000 & Fujifilm \\
ImageJ & NIH \\
ImageStudio, ImageStudio Lite 5.2.5 & LI-COR \\
Inkscape & Open Source Graphics \\
Lasergene SeqBuilder Pro & DNASTAR \\
LSM 510 Release Version 4.0 SP2 & Zeiss \\
LSM Image Browser & Zeiss \\
MARS data anaylsis software & BMG labtech \\
Microsoft Office 2016 & Microsoft \\
NanoDrop 2000 Software & ThermoScientific \\
SnapGene 5.0 & GSL Biotech LLC \\
\hline \hline
\end{tabular}

\subsubsection{Consumable}

\section{Table 3: Consumables}

\begin{tabular}{|c|c|}
\hline Consumable & Supplier \\
\hline 96 well micro test plate & Sarstedt \\
\hline 96 well micro test plate lid & Sarstedt \\
\hline Amersham Hybond ECL Nitrocellulose Blotting Membrane & GE Healthcare \\
\hline Amersham Hyperfilm ${ }^{\mathrm{TM}}$ ECL & GE Healthcare \\
\hline Amersham Protran 0.45 um NC Nitrocellulose Blotting Membrane & GE Healthcare \\
\hline Amicon ${ }^{\circledR}$ Ultra Centrifugal Filters, Ultracel ${ }^{\circledR}$ & Millipore \\
\hline Bottle Top Filter, 0.1 um & Sarstedt \\
\hline Bottle Top Filter, 0.2 um & Sarstedt \\
\hline Casy cups with lids & Roche \\
\hline Cell culture dish $10 \mathrm{~cm}$ & Sarstedt \\
\hline Cell culture dish $15 \mathrm{~cm}$ & Sarstedt \\
\hline Centrifuge Bottle Assembly, Polycarbonate $50 \mathrm{ml}$ & Beckman Coulter \\
\hline Centrifuge tube, thick wall, Polycarbonate 500 ul & Beckman Coulter \\
\hline Cryotubes (Nunc) & ThermoFisher Scientific \\
\hline Cuvette & Sarstedt \\
\hline Falcon $15 \mathrm{ml}$ & Sarstedt \\
\hline Falcon $50 \mathrm{ml}$ & Sarstedt \\
\hline Filter tips $10,20,200,1000 \mathrm{ul}$ & Greiner \\
\hline Inoculation spreader glass beads & ThermoFisher Scientific \\
\hline Medix XBU medical X-ray film & FOMA Bohemia \\
\hline Micro-reaction tubes $(1.5,2 \mathrm{ml})$ & Sarstedt \\
\hline Micro-reaction tubes safe lock $(0.2,0.5 \mathrm{ml})$ & Eppendorf \\
\hline Micro-reaction tubes safe lock $(1.5,2 \mathrm{ml})$ & Sarstedt \\
\hline Microscope coverslips (12 mm Ø) & Menzel-Glaeser \\
\hline Microscope slides & Menzel-Glaeser \\
\hline Mini Protean TGX Precast gels (4-20\%) & Bio-Rad \\
\hline Parafilm "M" & Bemis Company, Inc. \\
\hline PD-10 desalting columns & GE Healthcare \\
\hline
\end{tabular}


Petri dish $10 \mathrm{~cm}$

Plastic Pipet tips $5 \mathrm{ml}, 10 \mathrm{ml}, 25 \mathrm{ml}$

Polystyrene Round-Bottom tubes $13 \mathrm{ml}$

Polystyrene Round-Bottom tubes $5 \mathrm{ml}$

Slide-A-lyzer dialysis membrane

Spin-XR concentrators

Syringe filter $0.2 \mathrm{um}$

Syringe filter $0.4 \mathrm{um}$

Syringes/ needles

TC-Plate 24 well standard

TC-Plate 6 well standard

VIVASPIN concentrator

Whatman gel blotting paper
Sarstedt

Greiner

Sarstedt

BD Biosciences

ThermoFisher Scientific

Corning

Th. Geyer

Th. Geyer

B. Braun, Servoprax

Sarstedt

Sarstedt

Sartorius

GE Healthcare

\subsubsection{Kit}

Table 4: Kits

\begin{tabular}{ll}
\hline \hline Kit & Supplier \\
\hline \hline Duolink ${ }^{\circledR}$ In Situ Detection Reagents Red & Sigma-Aldrich \\
Duolink ${ }^{\circledR}$ In Situ Kit & Sigma-Aldrich \\
NucleoBond $^{\mathrm{TM}}$ Xtra Midi & Macherey-Nagel \\
NucleoSpin ${ }^{\circledR}$ Gel and PCR Clean-up & Macherey-Nagel \\
NucleoSpin ${ }^{\circledR}$ Plasmid & Macherey-Nagel \\
Pierce ${ }^{\circledR}$ BCA Protein Assay Kit & ThermoFisher Scientific \\
\hline \hline
\end{tabular}

\subsubsection{Enzyme}

Table 5: Enzymes

\begin{tabular}{ll}
\hline \hline Enzyme & Supplier \\
\hline \hline Creatine phosphokinase & CALBIOCHEM \\
DNaseI & Applichem \\
Fast alkaline phosphatase (FastAP) & ThermoFisher Scientific \\
Phusion High-Fidelity DNA Polymerase & ThermoFisher Scientific \\
Restriction enzymes & ThermoFisher Scientific \\
T4 DNA ligase & ThermoFisher Scientific \\
Gibco ${ }^{\circ}$ Trypsin/ EDTA $0.25 \%(1 \mathrm{x})$ & Invitrogen \\
\hline \hline
\end{tabular}

\subsubsection{Chemical}

Table 6: Chemicals

\begin{tabular}{ll}
\hline \hline Chemical & Supplier \\
\hline \hline Mercaptoethanol & Roth \\
Acetic acid & Roth \\
Acrylamide 4K Solution (30\%) & AppliChem \\
Adenosine 5'-triphosphate disodium salt hydrate (A3377) & Sigma-Aldrich \\
Agarose PeqGold & VWR \\
Amylose Resin High Flow & New England BioLabs \\
Aluminum-sulfate-(14-18)-hydrate & AppliChem \\
Ampicillin & Roth \\
Ammonium persulfate & Sigma-Aldrich \\
Aprotinin & Roth \\
Bovine Serum Albumin (BSA) & ThermoFisher Scientific \\
Bromophenol blue & Serva \\
BSA, fraction V & AppliChem \\
Calcium chloride (CaCl2) & Sigma-Aldrich \\
Chloramphenicol & Sigma-Aldrich \\
Cold water fish gelatin & Sigma-Aldrich \\
Coomassie Brilliant Blue (CBB)-G250 & Serva \\
\hline
\end{tabular}


Cyanogen bromide-activated Sepharose 4B

DAPI (D9542)

Dithiothreitol (DTT)

Digitonin

Disodium hydrogen phosphate (Na2HPO4)

dNTP Set, $100 \mathrm{mM}$ solutions

Ethanol (analytical grade)

Ethylenediaminetetraacetic acid (EDTA)

Ethyleneglycol-bis (b-aminoethyl ether)-N,N,N',N'-tetraacetic acid (EGTA)

Fetal bovine serum (FBS) Superior

Formaldehyde Solution min. 37\%

GeneRuler 100bp DNA Ladder

GeneRuler 1kb DNA Ladder

GFP-Trap ${ }^{\circledR}$

Gibco® Dulbecco's Modified Eagle Medium (DMEM, 1x)

Gibco® Opti-MEM Reduced Serum Media (1x)

Gibco ${ }^{\circledR}$ Penicillin Streptomycin

Glycerol 87\%

Glutathione Sepharose 4 Fast Flow

Glutathione Sepharose High Performance

HEPES

Hydrochloric acid ( $\mathrm{HCl})$

Imidazole

ImmobilonTM Western Chemiluminescent HRP Substrate

IPTG

Kanamycin sulfate

LB medium

LB Agar

Leptomycin B

Leupeptin

Lipofectamine ${ }^{\circledR} 2000$

Lipofectamine ${ }^{\circledR}$ RNAiMAX

Magnesium acetate $\left(\mathrm{Mg}(\mathrm{OAc})_{2}\right)$

Magnesium Chloride $\left(\mathrm{MgCl}_{2}\right)$

Methanol (technical grade)

Mowiol ${ }^{\circledR}$ 4-88

NeutrAvidin ${ }^{\circledR}$ Agarose ??

Ni-NTA Agarose

Nonidet P-40 (NP-40)

Oligonucleotides

Ortho-Phosphoric acid 85\% p.A.

PageRuler Plus Prestained Protein Ladder

PageRuler Prestained Protein Ladder

PageRuler Unstained Protein Ladder

Poly-L-lysine solution $0.1 \%(\mathrm{w} / \mathrm{v})$

Protein A-Agarose

Pepstatin

Phenylmethane sulfonyl fluoride (PMSF)

Pierce Coomassie Plus Protein Assay Reagent

Ponceau S

Potassium acetate (KOAc)

Potassium chloride ( $\mathrm{KCl}$ )

Potassium dihydrogen phosphate $\left(\mathrm{KH}_{2} \mathrm{PO}_{4}\right)$

Milk powder

Protein-A-Sepharose 4 Fast Flow

RFP-Trap ${ }^{\circledR}$

RNase free water

SafeViewTM Classic

Sodium acetate $(\mathrm{NaOAc})$

Sodium azide (NaN3)

Sodium chloride $(\mathrm{NaCl})$

Sodium deoxycholate
Sigma-Aldrich

Sigma-Aldrich

AppliChem

Calbiochem

Sigma-Aldrich

Thermo Fisher

Roth

AppliChem

AppliChem

Biochrom

Millipore

ThermoFisher Scientific

ThermoFisher Scientific

Chromotek

Invitrogen

Invitrogen

Invitrogen

AppliChem

GE Healthcare

GE Healthcare

AppliChem

AppliChem

Roth

Millipore

ThermoFisher Scientific

Roth

AppliChem

AppliChem

Enzo Life Sciences

Roth

Invitrogen

Invitrogen

Roth

Roth

Roth

Calbiochem

ThermoFisher Scientific

Qiagen

AppliChem

Sigma-Aldrich

AppliChem

ThermoFisher Scientific

ThermoFisher Scientific

ThermoFisher Scientific

Sigma-Aldrich

Roche

Roth

AppliChem

ThermoFisher Scientific

Sigma-Aldrich

Roth

AppliChem

Roth

Roth

GE Healthcare

Chromotek

Santa Cruz Biotechnology

Applied Biological Materials Inc.

Roth

Merck

Roth

Merck 
Sodium dodecyl sulfate (SDS), powder

Sodium dodecyl sulfate (SDS), 20\% liquid

TEMED

Tris (buffer grade)

Triton-X100

Trypan blue solution $0.4 \%$

Tween
Roth

AppliChem

Roth

AppliChem

Sigma-Aldrich

Sigma-Aldrich

Roth

\subsubsection{Bacterial media}

Table 7: Bacterial media

\begin{tabular}{ll}
\hline \hline Media & Constituantes \\
\hline \hline 2YT & $1.6 \%(\mathrm{w} / \mathrm{v})$ tryptone, $1 \%(\mathrm{w} / \mathrm{v})$ yeast extract, $0.5 \%(\mathrm{w} / \mathrm{v}) \mathrm{NaCl}, \mathrm{pH} 7.0$ \\
LB & $1 \%(\mathrm{w} / \mathrm{v})$ bacto-tryptone, $0.5 \%(\mathrm{w} / \mathrm{v})$ yeast extract, $1 \%(\mathrm{w} / \mathrm{v}) \mathrm{NaCl}, \mathrm{pH} 7.0$ \\
LB agar plates & $\mathrm{LB}$ supplemented with $1.5 \%(\mathrm{w} / \mathrm{v})$ bacto-agar \\
SOC medium & $2 \%(\mathrm{w} / \mathrm{v})$ tryptone, $0.5 \%(\mathrm{w} / \mathrm{v})$ yeast extract, $10 \mathrm{mM} \mathrm{NaCl,}, 2.5 \mathrm{mM} \mathrm{KaCl,} 10$ \\
& $\mathrm{mM} \mathrm{MgCl}, 10 \mathrm{mM} \mathrm{MgSO} 4,0.36 \%(\mathrm{w} / \mathrm{v})$ glucose, $\mathrm{pH} 7.0$ \\
Export complex buffer & $20 \mathrm{mM} \mathrm{Tris-Cl,} \mathrm{pH} 7.4,150 \mathrm{mM} \mathrm{NaCl,2} \mathrm{mM} \mathrm{Mg}(\mathrm{OAc})_{2}, 1 \mathrm{mM} \mathrm{DTT}$ \\
Western blot buffer $(1 \mathrm{x})$ & $25 \mathrm{mM}$ Tris-Cl, $193 \mathrm{mM}$ glycine, $0.1 \% \mathrm{SDS}$ \\
\hline \hline
\end{tabular}

\subsubsection{Stock solutions and buffers}

\subsubsection{Stock Solution}

Table 8: Stock solutions

\begin{tabular}{|c|c|}
\hline Stock Solutions & Constituantes \\
\hline Ampicillin & $100 \mathrm{mg} / \mathrm{ml}$ in water \\
\hline Aprotinin (AP) & $1 \mathrm{mg} / \mathrm{ml}$ in $20 \mathrm{mM}$ Hepes $\mathrm{pH} 7.4$ \\
\hline Ammonium persulfate (APS) & $10 \%$ APS (Sigma) in water \\
\hline Adenosine triphosphate (ATP) & $20 \mathrm{mM}$ Hepes, $\mathrm{pH}$ 7.4, $100 \mathrm{mM}$ ATP, $100 \mathrm{mM} \mathrm{Mg}(\mathrm{OAc})_{2}$ \\
\hline Calcium chloride $\left(\mathrm{CaCl}_{2}\right)$ & $250 \mathrm{mM} \mathrm{CaCl}_{2}$ in water \\
\hline Chloramphenicol & $30 \mathrm{mg} / \mathrm{ml}$ in water \\
\hline Creatine phosphokinase & $2000 \mathrm{U} / \mathrm{ml}, 20 \mathrm{mM}$ Hepes, pH 7.4, 50\% glycerol \\
\hline Creatine phosphate & $80 \mathrm{mg} / \mathrm{ml}$ in water \\
\hline Dimethyl sulfoxide (DMSO) & $2.5 \mathrm{mM}$ in $\mathrm{EtOH}$ \\
\hline Digitonin & $10 \%(\mathrm{w} / \mathrm{v})$ in $\mathrm{DMSO}$ \\
\hline DTT (1,4-Dithiothreitol) & $1 \mathrm{M}$ in water \\
\hline EDTA & $500 \mathrm{mM}, \mathrm{pH} 8.0$ in water \\
\hline EGTA & $500 \mathrm{mM}, \mathrm{pH} 8.0$ in water \\
\hline FITC-Phalloidin & $0.2 \mathrm{mg} / \mathrm{mL}$ in methanol \\
\hline $\begin{array}{l}\text { Hepes } 4 \text {-(2-hydroxyethyl)-1- } \\
\text { piperazineethanesulfonic acid }\end{array}$ & $238,3 \mathrm{mM}$ in water ?? \\
\hline $\begin{array}{l}\text { Isopropyl } \beta-D-1- \\
\text { thiogalactopyranoside (IPTG) }\end{array}$ & $1 \mathrm{M}$ in water \\
\hline Kanamycin & $60 \mathrm{mg} / \mathrm{ml}$ in water \\
\hline Leupeptin & $1 \mathrm{mg} / \mathrm{ml}$ in $\mathrm{DMSO}$ \\
\hline Leptomycin B & $10 \mathrm{mM}$ in $100 \% \mathrm{EtOH}$ \\
\hline Pepstatin & $1 \mathrm{mg} / \mathrm{ml}$ in DMSO \\
\hline $\begin{array}{l}\text { Phenylmethylsulfonyl fluoride } \\
\text { (PMSF) }\end{array}$ & $100 \mathrm{mM}$ in 2-propanol \\
\hline
\end{tabular}




\subsubsection{Buffers}

Table 9: Buffers

\begin{tabular}{|c|c|}
\hline Buffer & "Constituantes \\
\hline Anisotropy assay buffer & $\begin{array}{l}20 \mathrm{mM} \text { Tris-Cl, } \mathrm{pH} 7.4,130 \mathrm{mM} \mathrm{NaCl}, 2 \mathrm{mM} \mathrm{Mg}(\mathrm{OAc})_{2}, 1 \mathrm{mM} \text { DTT, } 0.005 \% \\
\text { digitonin }\end{array}$ \\
\hline Binding buffer & $\begin{array}{l}50 \mathrm{mM} \text { Tris-Cl, pH 7.4, } 200 \mathrm{mM} \mathrm{NaCl}, 1 \mathrm{mM} \mathrm{MgCl}_{2}, 5 \% \text { glycerol, } 1 \mathrm{mM} \text { DTT, } \\
20 \mathrm{mg} / \mathrm{ml} \mathrm{BSA}\end{array}$ \\
\hline Coomassie buffer & $\begin{array}{l}0.5 \% \mathrm{G} 250 \text { Coomassie brilliant blue, } 0.25 \% \text { R2 } 50 \text { Coomassie brilliant blue in } \\
100 \% \mathrm{EtOH}\end{array}$ \\
\hline DNA loading buffer & $0.2 \%$ bromophenol blue, $0.2 \%$ xylene cyanole, $60 \%$ glycerol, $60 \mathrm{mM}$ EDTA \\
\hline Export complex buffer & $20 \mathrm{mM}$ Tris-Cl, $\mathrm{pH} 7.4,150 \mathrm{mM} \mathrm{NaCl}, 2 \mathrm{mM} \mathrm{Mg}(\mathrm{OAc})_{2}, 1 \mathrm{mM}$ DTT \\
\hline Fixer buffer & $10 \%$ ethanol, $5 \%$ acetic acid \\
\hline HBS buffer $(2 x)$ & $50 \mathrm{mM}$ Hepes, $250 \mathrm{mM} \mathrm{NaCl}, 1.5 \mathrm{mM} \mathrm{Na}_{2} \mathrm{HPO}_{4}$, pH 6.94 \\
\hline HisTrap buffer A & $\begin{array}{l}50 \mathrm{mM} \text { Hepes, pH 7.5, } 500 \mathrm{mM} \mathrm{NaCl}, 3 \mathrm{mM} \text { imidazole, } 5 \mathrm{mM} \mathrm{MgCl}_{2}, 10 \% \\
\text { glycerol, } 1 \mathrm{mM} \text { DTT, } 30 \mu \mathrm{M} \text { GTP }\end{array}$ \\
\hline HisTrap buffer B & $\begin{array}{l}50 \mathrm{mM} \text { Hepes, } \mathrm{pH} \text { 7.5, } 500 \mathrm{mM} \mathrm{NaCl}, 500 \mathrm{mM} \text { imidazole, } 5 \mathrm{mM} \mathrm{MgCl} 2,10 \\
\% \text { glycerol, } 1 \mathrm{mM} \mathrm{DTT,} 30 \mu \mathrm{M} \text { GTP }\end{array}$ \\
\hline HisTrap desalting buffer & $\begin{array}{l}50 \mathrm{M} \text { Hepes, } \mathrm{pH} 7.5,500 \mathrm{mM} \mathrm{NaCl}, 5 \mathrm{mM} \mathrm{MgCl} 2,10 \% \text { glycerol, } 1 \mathrm{mM} \\
\text { DTT, } 30 \mu \mathrm{M} \text { GTP }\end{array}$ \\
\hline HisTrap Lysis buffer & $\begin{array}{l}50 \mathrm{mM} \text { Hepes, } \mathrm{pH} \text { 7.5, } 500 \mathrm{mM} \mathrm{NaCl}, 3 \mathrm{mM} \text { imidazole, } 5 \mathrm{mM} \mathrm{MgCl}_{2}, 10 \% \\
\text { glycerol, } 1 \mathrm{mM} \text { DTT, } 30 \mu \mathrm{M} \text { GTP, } 1 \text { Roche protease inhibitor tablet, } 100 \\
\mathrm{mg} / \mathrm{ml} \text { DNase I }\end{array}$ \\
\hline Laemmli buffer $(10 x)$ & $250 \mathrm{mM}$ Tris, $1.92 \mathrm{M}$ glycine, $0.5 \% \mathrm{SDS}$ \\
\hline MBP-binding buffer & $\begin{array}{l}20 \mathrm{mM} \text { Hepes, } \mathrm{pH} 8.0,100 \mathrm{mM} \mathrm{KCl}, 10 \mathrm{mM} \mathrm{Mg}(\mathrm{OAc})_{2}, 10 \% \text { glycerol, } 0.01 \% \\
\text { TritonX-100, } 1 \mathrm{mM} \text { DTT }\end{array}$ \\
\hline MBP-lysis buffer & $\begin{array}{l}20 \mathrm{mM} \text { Hepes, } \mathrm{pH} 8.0,100 \mathrm{mM} \mathrm{KCl}, 10 \mathrm{mM} \mathrm{Mg}(\mathrm{OAc})_{2}, 10 \% \text { glycerol, } 0.01 \% \\
\text { TritonX-100, } 1 \mathrm{mM} \text { DTT, } 1 \mathrm{mM} \mathrm{AP,} 1 \mathrm{mM} \mathrm{LP}, 1 \mathrm{mM} \text { DTT, } 1 \mathrm{mM} \text { PMSF }\end{array}$ \\
\hline NP40 buffer & $\begin{array}{l}50 \mathrm{mM} \text { Tris-Cl, } \mathrm{pH} 8.8,150 \mathrm{mM} \mathrm{NaCl}, 1 \% \mathrm{NP} 40,1 \mu \mathrm{g} / \mu \mathrm{l} \mathrm{AP}, 1 \mu \mathrm{g} / \mu \mathrm{LP}, 1 \\
\text { mM DTT, } 1 \mathrm{mM} \text { PMSF }\end{array}$ \\
\hline PBS $(10 x)$ & 1.37 M NaCl, pH 7.5, $27 \mathrm{mM} \mathrm{KCl}, 100 \mathrm{mM} \mathrm{Na}_{2} \mathrm{HPO}_{4}, 18 \mathrm{mM} \mathrm{KH}_{2} \mathrm{PO}_{4}$ \\
\hline $\operatorname{PBS}(1 \mathrm{x})$ & $137 \mathrm{mM} \mathrm{NaCl}, \mathrm{pH}$ 7.5, $2.7 \mathrm{mM} \mathrm{KCl}, 10 \mathrm{mM} \mathrm{Na}_{2} \mathrm{HPO}_{4}, 1.8 \mathrm{mM} \mathrm{KH}_{2} \mathrm{PO}_{4}$ \\
\hline Ponceau S solution & $0.5 \%$ Ponceau $\mathrm{S}$ in $1 \%$ acetic acid \\
\hline Pulldown buffer & $50 \mathrm{mM}$ Tris-Cl, pH 7.4, $200 \mathrm{mM} \mathrm{NaCl}, 1 \mathrm{mM} \mathrm{MgCl}_{2}, 5 \%$ glycerol, $1 \mathrm{mM}$ DTT \\
\hline SDS sample buffer $(4 \mathrm{x})$ & 125 mM Tris-Cl, pH 6.8, 4\% SDS, $0.02 \%$ Bromophenol blue, $10 \%$ glycerol \\
\hline TAE buffer $(1 \mathrm{x})$ & $40 \mathrm{mM}$ Tris, $1 \mathrm{mM}$ EDTA, $0.114 \%$ acetic acid \\
\hline TAE buffer $(50 x)$ & $2 \mathrm{M}$ Tris-Cl, $0.05 \mathrm{M}$ EDTA, 5.71\% acetic acid \\
\hline TBS $(10 x)$ & $500 \mathrm{mM}$ Tris-Cl, $\mathrm{pH} 7.6 ; 1.5 \mathrm{M} \mathrm{NaCl}$ \\
\hline TBS $(1 \mathrm{x})$ & $50 \mathrm{mM}$ Tris-Cl, $\mathrm{pH} 7.6 ; 150 \mathrm{mM} \mathrm{NaCl}$ \\
\hline TBS-T (1x) & TBS $(1 \mathrm{x}), 0.1 \%$ Tween-20 \\
\hline
\end{tabular}




\begin{tabular}{ll}
\hline Transport buffer (10x) & $200 \mathrm{mM}$ Hepes, pH 7.3, $1.1 \mathrm{M} \mathrm{KOAc}, 20 \mathrm{mM} \mathrm{Mg}(\mathrm{OAc})_{2}, 10 \mathrm{mM}$ EGTA \\
Transport buffer (1x) & $20 \mathrm{mM}$ Hepes, pH 7.3, $110 \mathrm{mM} \mathrm{KOAc,} \mathrm{mM} \mathrm{Mg(OAc})_{2}, 1 \mathrm{mM}$ EGTA \\
Wash buffer A (PLA) & $10 \mathrm{mM}$ Tris-Cl, pH 8.0, $150 \mathrm{mM} \mathrm{NaCl}, 0.05 \%$ Tween-20 \\
Wash buffer B (PLA) & $200 \mathrm{mM}$ Tris-Cl, pH 7.5, $100 \mathrm{mM} \mathrm{NaCl}$ \\
Western blot buffer (10x) & $250 \mathrm{mM}$ Tris-Cl, $1.93 \mathrm{M}$ glycine, 0.2\% SDS \\
Western blot buffer (1x) & $25 \mathrm{mM}$ Tris-Cl, $193 \mathrm{mM}$ glycine, $0.1 \%$ SDS \\
\hline \hline
\end{tabular}

\subsubsection{Mammalian cell line}

Table 10: Mammalian cell lines

\begin{tabular}{ll}
\hline \hline Cell line & Description \\
\hline \hline HeLa P4 & Human adenocarcinoma cell line that expresses CD4; derived from cervix of a \\
& 31-year-old woman (Charneau et al., 1994) \\
293T HEK & Human embryonic kidney cell line transformed with adenovirus 5 DNA; \\
& derived from fetus (CRL-2828, ATCC; DuBridge et al., 1987) \\
Flp-InTM T-REx ${ }^{\text {TM }} 293$ & The cell Line contains a stably integrated pFRT/acZeo and pcDNA \\
& from the T-REx ${ }^{\text {TM }}$ System designed for generation of stably expressing cell \\
& lines (R78007, ThermoFisher Scientific; Weterman et al., 2001) \\
COS-7 & Monkey African green kidney cell line transformed with SV40 (87021302, \\
& Sigma-Aldrich; Gluzman et al., 1981) \\
\hline \hline
\end{tabular}

\subsubsection{Bacterial strain}

Table 11: Bacterial strains

\begin{tabular}{ll}
\hline \hline Strain & Description \\
\hline \hline BL21 (DE3) & F- ompT hsdS $\mathrm{B}_{\mathrm{B}}(\mathrm{rB}-\mathrm{mB}-$ ) gal dcm (DE3) \\
BL21 (DE3) pLysS & F- ompT hsdS $\mathrm{B}$ (rB- mB-) gal dcm (DE3) pLysS (CamR) \\
BL21 (DE3) codon+ & F- ompT hsdS(rB- mB-) dem+ Tetr gal 1 (DE3) endA Hte [argU proL Camr] \\
& LEMO21 (DE3) fhuA2 [lon] ompT gal (lDE3) [dcm] hsdS/ pLemo(CamR) \\
DH5 $\alpha$ & F- \$80lacZ $\Delta$ M15 $\Delta$ (lacZYA-argF) U169 recA1 endA1 hsdR17 (rK-,mK+) \\
& phoA supE44 $\lambda$ - thi-1 gyrA96 relA1 \\
\hline \hline
\end{tabular}

\subsubsection{Antibodies}

\subsubsection{Primary antibodies}

Table 12: Primary antibodies

\begin{tabular}{llllll}
\hline \hline Number & Name & Dilution & Application & Species & Supplier \\
\hline \hline 004 & $\alpha$-CRM1 & $1: 500$ & Western blot/ & Goat & Kehlenbach et al., 1999 \\
& & & Immunofluorescence & & \\
& & & &
\end{tabular}




\begin{tabular}{|c|c|c|c|c|c|}
\hline 114 & $\alpha$-CRM1 & $1: 1000$ & $\begin{array}{l}\text { Western blot/ } \\
\text { Immunofluorescence }\end{array}$ & Rabbit & Kehlenbach et al., 1999 \\
\hline 121 & $\alpha$-FLAG & $1: 3000$ & Immunofluorescence & Mouse & Sigma-Aldrich \\
\hline 080 & $\alpha$-GAPDH & $1: 1000$ & Western blot & Rabbit & Proteintech \\
\hline 015 & $\alpha-$ GFP (3H9) & $1: 1000$ & Western blot & Rat & Chromotek \\
\hline 134 & $\alpha-\mathrm{HA}$ & $1: 500$ & Immunofluorescence & Rabbit & Sigma-Aldrich \\
\hline 140 & $\alpha$-His & $1: 1000$ & Western blot & Mouse & Qiagen \\
\hline 191 & $\alpha$-myc & $1: 200$ & Immunofluorescence & Mouse & Santa Cruz \\
\hline 314 & $\alpha-$ myc $(\mathrm{A}-14)$ & $1: 300$ & Immunofluorescence & Rabbit & Santa Cruz \\
\hline 039 & $\alpha$-Nup214 'F' & $1: 500$ & Western blot & Rabbit & Kehlenbach et al., 1999 \\
\hline 039 & $\alpha-N u p 214$ 'F' & $1: 200$ & Immunofluorescence & Rabbit & Kehlenbach et al., 1999 \\
\hline 404 & $\alpha$-Nup214 & $1: 300$ & Western Blot & Rabbit & Kehlenbach et al., 1998 \\
\hline 280 & $\alpha$-Nup62 & $1: 1000$ & $\begin{array}{l}\text { Western blot/ } \\
\text { Immunofluorescence }\end{array}$ & Rabbit & Sigma-Aldrich \\
\hline 046 & $\alpha$-Nup88 & $1: 1000$ & $\begin{array}{l}\text { Western blot/ } \\
\text { Immunofluorescence }\end{array}$ & Mouse & BD Pharmingen \\
\hline 053 & $\alpha$-Nup358 & $1: 1000$ & Immunofluorescence & Goat & Melchior et al., 2004 \\
\hline 081 & $\alpha$-Tubulin & $1: 500$ & Western blot & Rabbit & Proteintech \\
\hline
\end{tabular}

\subsubsection{Secondary antibodies}

Table 13: Secondary antibodies

\begin{tabular}{lllll}
\hline \hline Name & Dilution & Use & Species & Supplier \\
\hline \hline$\alpha$-goat AlexaFluor ${ }^{\circledR} 488$ & $1: 1000$ & Immunofluorescence & Donkey & Molecular Probes \\
$\alpha$-goat AlexaFluor ${ }^{\circledR} 594$ & $1: 1000$ & Immunofluorescence & Donkey & Molecular Probes \\
$\alpha$-mouse AlexaFluor ${ }^{\circledR} 488$ & $1: 1000$ & Immunofluorescence & Donkey & Molecular Probes \\
$\alpha$-mouse AlexaFluor ${ }^{\circledR} 594$ & $1: 1000$ & Immunofluorescence & Donkey & Molecular Probes \\
$\alpha$-rabbit AlexaFluor ${ }^{\circledR} 488$ & $1: 1000$ & Immunofluorescence & Donkey & Molecular Probes \\
$\alpha$-rabbit AlexaFluor ${ }^{\circledR} 594$ & $1: 1000$ & Immunofluorescence & Donkey & Molecular Probes \\
$\alpha$-mouse IgG 680 & $1: 1000$ & Western blot & Donkey & LI-COR \\
$\alpha$-mouse IgG 800 & $1: 1000$ & Western blot & Donkey & LI-COR \\
$\alpha$-mouse IgG2 680 & $1: 1000$ & Western blot & Donkey & LI-COR \\
$\alpha$-rabbit IgG 680 & $1: 1000$ & Western blot & Donkey & LI-COR \\
$\alpha$-rabbit IgG 800 & $1: 1000$ & Western blot & Donkey & LI-COR \\
$\alpha$-rat IgG 800 & $1: 1000$ & Western Blot & Donkey & LI-COR \\
$\alpha$-goat IgG & $1: 1000$ & Western blot & Donkey & LI-COR \\
\hline \hline
\end{tabular}

\subsubsection{Synthesized peptides}

Fluorescently tagged peptides were synthesized by EMC microcollections (Tübingen,

Germany). The HPLC results yielded a high purity level of $\geq 90 \%$. The molecular weight was determined by Electrospray ionization (ESI) mass spectroscopy and used to calculate the 
molar concentration of each peptide. The peptides were all tagged with 6-carboxyfluorescein (6-FAM) on their lysine residues. The peptides were provided in powdered form and reconstituted to the desired molar concentration using autoclaved water.

Table 14: Synthesized peptides

\begin{tabular}{ll}
\hline \hline Peptide & Sequence \\
\hline \hline fNup214-NES & LNHLVDSLQQLRLK (FAM\#) \\
fPKI-NES & LSNELALKLAGLDIK (FAM\#) \\
fHIV-rev-NES & LQLPPLERLTLK (FAM\#) \\
\hline \hline
\end{tabular}

\subsubsection{Oligonucleotides and siRNAs}

Oligonucleotides were ordered from Sigma-Aldrich with a concentration of $100 \mu \mathrm{M}$ in water, a synthesis scale of $0.025 \mathrm{umol}$ and desalted purification grade.

Oligonucleotides used for cloning were designed with restriction sites at their 5 '-ends, in addition to a 5 '-end overhang to guarantee high cleavage efficiency by the restriction enzymes. Oligonucleotides were generated using SeqBuilder software (Lasergene) and their melting temperatures were calculated using the Tm Calculator tool available online from ThermoFisher Scientific (https://www.thermofisher.com/de/de/home/brands/thermoscientific/molecular-biology/molecular-biology-learning-center/molecular-biology-resourcelibrary/thermo-scientific-web-tools/tm-calculator.html).

\subsubsection{Oligonucleotide for cloning}

Table 15: Oligonucleotides for cloning

\begin{tabular}{ll}
\hline \hline Primer & Sequence \\
\hline \hline Nup88fl_ApIII_For & TTTCTTAAGGCGGCCGCC \\
HA_KpnI_Rev & TTTGGTACCTCATCCTGCATAGTCTGG \\
Nup214_fl_PfI23II_fw & TTTCGTACGGCCACCATGGGAGACGAGATGGATGCC \\
Nup214_fl_XmaJI_rev & TTTCCTAGGGCTTCGCCAGCCACCAAAACCC \\
Nup214_f586_HindIII & AAAAAAGCTTAACCTTAGTGAAAAGTTTACTGCTG \\
Nup214_r1085_6xHis_EcoRI & TTTGAATTCTCAGTGATGGTGATGGTGATGGGGGTGGGAAG \\
Nup214_f650_HindIII & GACTAGG \\
Nup214_r950_6xHis_EcoRI & AAAAAAGCTTCGATCTGCTCAGGGCAGT \\
Nup214_f680_Acc65i & TTTGAATTCTCAGTGATGGTGATGGTGATGGAAGTTTCTCAG \\
Nup214_r1209_6xHis_EcoRI & TTGTGCCTG \\
Nup214_f801_EcoRI & AAAAGGTACCCAGGCAAAGTCACTTCAG \\
\hline
\end{tabular}




\begin{tabular}{|c|c|}
\hline Nup214_fw_RT-PCR_P1 & AACAAGGACCGTCTCGCATT \\
\hline Nup214_rev_RT-PCR_P1 & CATGACCCAAGCCAAAGCAC \\
\hline Nup214_fw_RT-PCR_P2 & GTTCAGCAACTACCCCCTCC \\
\hline Nup214_rev_RT-PCR_P2 & CTGTCACCTGGCTTCTCAGG \\
\hline Nup88_fw_RT-PCR_P1 & CTGTGAATTGCCCACCCTGA \\
\hline Nup88_rev_RT-PCR_P1 & CTTGGATAAGCCCCAAGCGA \\
\hline Nup88_fw_RT-PCR_P2 & TCTGCTTAGAAATGGCTGAGTGA \\
\hline Nup88_rev_RT-PCR_P2 & TCACGAGTGTTCCTGTGCTT \\
\hline Nup62_fw_RT-PCR_P1 & TCGGAACTTAGTGGCACCAG \\
\hline Nup62_rev_RT-PCR_P1 & ATGCTTGGGATACAACCGCT \\
\hline Nup62_fw_RT-PCR_P2 & CCAGGAGCTCGACTTCATCC \\
\hline Nup62_rev_RT-PCR_P2 & GCTCCTTGACCAACTCCTCC \\
\hline GAPDH_fd & CAGCCTCAAGATCATCAGCAATG \\
\hline GAPDH_rev & GTCTTCTGGGTGGCAGTGATG \\
\hline Nup214 (801-950)_PaeI_fwd & TTTGCATGCCCACTGGATCCCAAGAGTGA \\
\hline Nup214(801-950)_AvrII_rev & TTTCCTAGGTCAGAAGTTTCTCAGTTG \\
\hline CRM1_SdaI_fwd & TTTCCTGCAGGATGCCTGCAATTATGACC \\
\hline CRM1_AvrII_rev & TTTCCTAGGTCAGTGATGGTGATGGTGAT \\
\hline RanQ69L (1-180)_PaeI_fwd & TTTGCATGCGCTGCGCAGGGAGAG \\
\hline RanQ69L (1-180)_AvrII_rev & TTTCCTAGGGGGCTTGGATATAATA \\
\hline Nup214 (1916-2033)_PaeI_fwd & TTTGCATGCTCAAATACCTCTAACCTA \\
\hline Nup214 (1916-2033)_AvrII_rev & TTTCCTAGGTTATGTGGTGTTGCTGCT \\
\hline Nup214 (801-950) Forward PaeI & AAAAGCATGCCCACTGGATCCCAAGAGTGA \\
\hline Nup214 (801-950) Reverse AvrII & AAAACCTAGGTCAGAAGTTTCTCAGTTG \\
\hline CRM1 Forward SdaI & AAAACCCCTGCAGGATGCCTGCAATTATGACCATGC \\
\hline CRM1 Reverse AvrII & AAAACCCCTAGGTCAGTGATGGTGATGGTGAT \\
\hline Nup214 (1916-2033) Forward PaeI & TTTGCATGCATGTCAAATACCTCTAACCTATTTGGAAACAG \\
\hline Nup214 (1916-2033) Reverese AvrII & TTTCCTAGGTTATGTGGTGTTGCTGCTGCTCC \\
\hline RevNES_fwd_BglII/EcoRI & GATCTCTACAACTACCACCACTAGAACGACTGACACTAG \\
\hline RevNES_rev_BglII/EcoRI & AATTCTAGTGTCAGTCGTTCTAGTGGTGGTAGTTGTAGA \\
\hline Nup214NES_fwd_BglII/EcoRI & GATCTCTGGTGGATAGTCTTCAGCAGCTCCGCCTTG \\
\hline Nup214NES_rev_BglII/EcoRI & AATTCAAGGCGGAGCTGCTGAAGACTATCCACCAGA \\
\hline Nup88(585-651)_fwd_PaeI & AAAAGCATGCATGGAGGAGATTCAGCGGAGG \\
\hline Nup88(585-651)_rev_AvrII & AAAACCTAGGTCAGAGCTCAGAGTGAAAACTGTG \\
\hline Nup62(328-458)_fwd_PaeI & AAAAGCATGCATGACCTACGCGCAGCTGGAG \\
\hline Nup62 (328-458)_rev_AvrII & AAAACCTAGGTCACAGGTGCTCGATGATGTC \\
\hline RevNES_Stop_PspOMI_MfeI_fwd & $\begin{array}{l}\text { GGCCCAACTACAACTACCACCACTAGAACGACTGACACTAT } \\
\text { AGC }\end{array}$ \\
\hline RevNES_Stop_PspOMI_MfeI_rev & $\begin{array}{l}\text { AATTGCTATAGTGTCAGTCGTTCTAGTGGTGGTAGTTGTAGT } \\
\text { TG }\end{array}$ \\
\hline Nup214NES_Stop_PspOMI_MfeI_fwd & GGCCCAACTGGTGGATAGTCTTCAGCAGCTCCGCCTTTAGC \\
\hline
\end{tabular}




\begin{tabular}{ll}
\hline Nup214NES_Stop_PspOMI_MfeI_rev & AATTGCTAAAGGCGGAGCTGCTGAAGACTATCCACCAGTTG \\
CRM1_SdaI_fwd & AAAACCCCTGCAGGAAATGCCTGCAATTATGACC \\
CRM1_AvrII_rev & AAAACCCCTAGGTCAGGATCTGGATCTATCACAC \\
Nup214_1916_PaeI_fwd & TTTGCATGCAATCAAATACCTCTAACCTA \\
Nup214_801_PaeI_fwd & AAAAGCATGCAACCACTGGATCCCAAG \\
Nup62_328_PaeI_fwd & TTTGCATGCAAACCTACGCGCAGCTGGAGAGC \\
Nup88_585_PaeI_fwd & TTTGCATGCAAGAGGAGATTCAGCGGAGG \\
SPN1_fwd_PaeI & TTTTTGCATGCAAATGGAAGAGTTGAGTCAGG \\
SPN1_rev_AvrII & TTTTTCCTAGGCTAATTCTCCATGAGGCATCC \\
FRB_fwd_EcoRI & TTTT GAATTC GAGATGTGGCATGAAGGCCTG \\
FRB_rev_SwaI & TTTT ATTTAAAT CTGCTTTGAGATTCGTCGGAAC \\
FRB_rev_BgIII & TTT AGATCT CTGCTTTGAGATTCGTCGGAAC \\
HA-FRB_SmaI & AAAACCCGGGTATCCATATGACGTTCCAG \\
HA-FRB_MunI & AAAACAATTGCTACTGCTTTGAGATTCGTC \\
ApaI_Nup214_f792 & GGGCCCCTGGTTATCTGCATTTGC \\
MfeI-stop codon_Nup214_r991 & CAATTGCTAGGAGACAGATGACGTTG \\
Nup214_rev_2090_no stop codon & TTTTGGGCCCAGCTTCGCCAGCCACCAAAAC \\
Nup214_fwd_451_EcoRI & TTTTGAATTCATGGCTGCAGCCCCTGCCTC \\
\hline \hline
\end{tabular}

1.5.13.2 Oligonucleotide for mutagenesis

Table 16: Oligonucleotides for mutagenesis

\begin{tabular}{ll}
\hline \hline Primer & Sequence \\
\hline \hline NupNES1_1_f & GAAGAGGCTGAATCACGCGGTGGATAGTGCTCAGCAGCTCC \\
& GCCTT \\
NupNES1_1_r & AAGGCGGAGCTGCTGAGCACTATCCACCGCGTGATTCAGCC \\
& TCTTC \\
Nup214-F & GTCCTGCAAGTCACGGAGACTGTGAAAGTATGTGCA \\
Nup214-R2 & TGCACATACTTTCACAGTCTCCGTGACTTGCAGGAC \\
\hline \hline
\end{tabular}

1.5.13.3 Oligonucleotide for sequencing

Table 17: Oligonucleotide for sequencing

\begin{tabular}{lll}
\hline \hline Primer & Sequence & Source \\
\hline \hline Nup214_f804 & GATCCCAAGAGTGAAGCTCAGC & Self-generated \\
pcDNA3.1-FP & CAAACAACAGATGGCTGGC & GATC \\
pcDNA3.1-RP & CTCTGGCTAACTAGAGAAC & GATC \\
pEGFP_C2-FP & GATCACATGGTCCTGCTG & GATC \\
pET-RP & CTAGTTATTGCTCAGCGG & GATC \\
pGEX-3 & GGAGCTGCATGTGTCAGAG & GATC \\
pGEX-5 & CTGGCAAGCCACGTTTGG & GATC \\
\hline
\end{tabular}




\begin{tabular}{lll}
\hline pGEX3-RP & TCAAGAATTATACACTCCG & GATC \\
pGEX5-FP & AACGTATTGAAGCTATCCC & GATC \\
pMalE & TCAGACTGTCGATGAAGC & GATC \\
pQE-FP & CGGATAACAATTTCACACAG & GATC \\
pQE-RP & GTTCTGAGGTCATTACTGG & GATC \\
\hline \hline
\end{tabular}

\subsubsection{4 siRNA}

Table 18: siRNAs

\begin{tabular}{ll}
\hline \hline Primer & Sequence \\
\hline \hline siNup214_2 & GGTGAGAATCTTTGACTCC \\
siNup214_4 & \\
siRanBP2-2 & GUCACGAAACAGUGAAAG \\
\hline \hline
\end{tabular}

\subsubsection{Vectors and plasmids}

\subsubsection{Vectors}

Table 19: Vectors

\begin{tabular}{|c|c|c|c|c|}
\hline Number & Vector & Tag & Application & Source \\
\hline 30 & pEGFP-C1 & GFP & Transfection & Clontech \\
\hline 51 & pcDNA $3.1(+)$ & none & Transfection & Invitrogen \\
\hline 46 & pGex-6P-1 & GST & Expression & Amersham \\
\hline 52 & pcDNA3.1(+)-HA & HA-Tag (C-terminal) & Transfection & Kehlenbach \\
\hline 53 & pcDNA3.1(+)-FLAG & FLAG-Tag (C-terminal) & Transfection & Kehlenbach \\
\hline 65 & pmCherry-N1 & mCherry & Transfection & Kehlenbach \\
\hline 66 & pmCherry-C1 & mCherry & Transfection & Kehlenbach \\
\hline 70 & pEGFP-N1 & EGFP & Transfection & Clontech \\
\hline 75 & pMal-PreScission & MBP & Expression & Sarah Port \\
\hline 84 & $\begin{array}{l}\text { pcDNA5-FRT-TO- } \\
\text { cGFP }\end{array}$ & GFP & Stable transfection & ThermoFisher Scientific \\
\hline 85 & pQLinkG & GST & Expression & $\begin{array}{l}\text { FM Hughson; Scheich et } \\
\text { al. (2007) }\end{array}$ \\
\hline 86 & $\mathrm{pQLinkN}$ & no tag & Expression & $\begin{array}{l}\text { FM Hughson; Scheich et } \\
\text { al. (2007) }\end{array}$ \\
\hline 87 & pQLinkH & His & Expression & $\begin{array}{l}\text { FM Hughson; Scheich et } \\
\text { al. (2007) }\end{array}$ \\
\hline 88 & pQLinkHM & His-MBP & Expression & $\begin{array}{l}\text { FM Hughson; Baker et al. } \\
\text { (2015) }\end{array}$ \\
\hline 91 & pOG44 & untagged & Stable transfection & ThermoFisher Scientific \\
\hline
\end{tabular}




\subsubsection{Available plasmids}

Table 20: Available plasmids

\begin{tabular}{lll}
\hline \hline Number & Vector & Source \\
\hline \hline 488 & pGFP-NES(REV)-pK-M9 & Christiane Spillner \\
832 & Myc-Nup214 fl siRNA resistant & SR \\
1214 & pEGFP-C1-NUP88WT & Birthe Fahrenkroog \\
1215 & pFlag CMV2-NUP88 & Birthe Fahrenkroog \\
1316 & pQE80-His-ZZ-RanQ69L(1-180) & T. Güttler \\
1481 & pcDNA4myc-Nup214-HisA & Christiane Spillner \\
1590 & pcDNA4-Nup214(L885A/L887A/L1464A/F1468A)-myc-His & Sarah Port \\
1606 & pNup214-mCherry siRNA resistant & Sarah Port \\
1611 & pNup214(1-1632)-mCh & Sarah Port \\
1612 & pmCh-Nup214(1-1632) & Sarah Port \\
1615 & pNup214(1633-2090)-mCh & Sarah Port \\
1616 & pmCh-Nup214(1633-2090) & Sarah Port \\
1627 & pNup214-EGFP & Sarah Port \\
1628 & pEGFP-Nup214 & Sarah Port \\
1630 & pcDNA4-Nup214-myc-His (NES1mut, siRNA resistant) & Sarah Port \\
1979 & pET21a-Hs-CRM1-His & Thomas Monecke
\end{tabular}

\subsubsection{Generated plasmids}

\section{Table 21: Generated plasmids}

\begin{tabular}{lll}
\hline \hline Number & Vector & Cloning \\
\hline \hline 1823 & pGex-6p-1-Nup214 (586-1085)-His & PCR template: \#47, Primers:1341(F),1342(R), cloned into \\
& & vector \#46 \\
1824 & pGex-6p-1-Nup214 (650-950)-His & PCR template: \#47, Primers:1343(F), 1344(R), cloned into \\
& & vector \#46 \\
1825 & pGex-6p-1-Nup214 (680-1209)-His & PCR template: \#47, Primers:1345(F),1346(R), cloned into \\
& & vector \#46 \\
1915 & pEGFP-C1-Nup214 (878-887) & Oligo annealing using G1524/G1525, inserted into vector \\
& & \#30 \\
1916 & pEGFP-C1-Nup214 (878-887) & Oligo annealing using G1498/G1499, inserted into vector \\
& mutant & \#30 \\
1919 & pcDNA5-FRT-TO-cGFP-Nup214 & PCR template: \#47, Primers:1799(F),1800(R), cloned into \\
& & vector \#84 \\
1920 & pcDNA5-FRT-TO-cGFP-Nup214 & PCR template: \#1630, Primers:1799(F),1800@, cloned into \\
& (NES1 mutant, siRNA resistant) & vector \#84
\end{tabular}




\begin{tabular}{|c|c|c|}
\hline 1949 & pEGFP-C1-Nup214 (585-1085) & $\begin{array}{l}\text { PCR template: \#47, Primers:1341(F),1342(R), cloned into } \\
\text { vector \#30 }\end{array}$ \\
\hline 1950 & pEGFP-C1-Nup214 (650-950) & $\begin{array}{l}\text { PCR template: \#47, Primers:1343(F),1344(R), cloned into } \\
\text { vector \#30 }\end{array}$ \\
\hline 1951 & pEGFP-C1-Nup214 (680-1209) & $\begin{array}{l}\text { PCR template: \#47, Primers:1345(F),1346(R), cloned into } \\
\text { vector \#30 }\end{array}$ \\
\hline 1952 & pEGFP-N1-Nup214 (585-1085) & $\begin{array}{l}\text { PCR template: \#47, Primers:1341(F),1342(R), cloned into } \\
\text { vector \#70 }\end{array}$ \\
\hline 1953 & pEGFP-N1-Nup214 (650-950) & $\begin{array}{l}\text { PCR template: \#47, Primers:1343(F),1344(R), cloned into } \\
\text { vector \#70 }\end{array}$ \\
\hline 1954 & pEGFP-N1-Nup214 (680-1209) & $\begin{array}{l}\text { PCR template: \#47, Primers:1345(F),1346(R), cloned into } \\
\text { vector \#70 }\end{array}$ \\
\hline 1955 & pcDNA3-Nup214 (585-1085)-HA & $\begin{array}{l}\text { PCR template: \#47, Primers:1341(F),1342(R), cloned into } \\
\text { vector \#30 }\end{array}$ \\
\hline 1956 & pcDNA3-Nup214 (650-950)-HA & $\begin{array}{l}\text { PCR template: \#47, Primers:1343(F),1344(R), cloned into } \\
\text { vector \#30 }\end{array}$ \\
\hline 1957 & pcDNA3-Nup214 (680-1209)-HA & $\begin{array}{l}\text { PCR template: \#47, Primers:1345(F),1346(R), cloned into } \\
\text { vector \#30 }\end{array}$ \\
\hline 1958 & pcDNA3-Nup214 (585-1085)-FLAG & $\begin{array}{l}\text { PCR template: \#47, Primers:1341(F),1342(R), cloned into } \\
\text { vector } \# 70\end{array}$ \\
\hline 1959 & pcDNA3-Nup214 (650-950)-FLAG & $\begin{array}{l}\text { PCR template: \#47, Primers:1343(F),1344(R), cloned into } \\
\text { vector \#70 }\end{array}$ \\
\hline 1960 & pcDNA3-Nup214 (680-1209)-FLAG & $\begin{array}{l}\text { PCR template: \#47, Primers:1345(F),1346(R), cloned into } \\
\text { vector \#70 }\end{array}$ \\
\hline 1961 & $\begin{array}{l}\text { pMal-PP-Nup214 (586-1085)-His } \\
\text { (L878A/L882A) }\end{array}$ & $\begin{array}{l}\text { PCR template: \#1453, Mutagenesis by primers: 1490(F), } \\
\text { 1491(R) }\end{array}$ \\
\hline 1962 & $\begin{array}{l}\text { pMal-PP-Nup214 (650-950)-His } \\
\text { (L878A/L882A) }\end{array}$ & $\begin{array}{l}\text { PCR template: \#1454, Mutagenesis by primers: 1490(F), } \\
\text { 1491(R) }\end{array}$ \\
\hline 1963 & $\begin{array}{l}\text { pMal-PP-Nup214 (680-1209)-His } \\
\text { (L878A/L882A) }\end{array}$ & $\begin{array}{l}\text { PCR template: \#1455, Mutagenesis by primers: 1490(F), } \\
\text { 1491(R) }\end{array}$ \\
\hline 1991 & pMal-PP-Nup214 (801-1085)-His & $\begin{array}{l}\text { PCR template: \#47, Primers:1342(F),1946(R), cloned into } \\
\text { vector \#75 }\end{array}$ \\
\hline 1992 & pMal-PP-Nup214 (801-950)-His & $\begin{array}{l}\text { PCR template: \#47, Primers:1344(F),1946(R), cloned into } \\
\text { vector \#75 }\end{array}$ \\
\hline 1993 & pMal-PP-Nup214 (801-1209)-His & $\begin{array}{l}\text { PCR template: \#47, Primers:1346(F),1946(R), cloned into } \\
\text { vector } \# 75\end{array}$ \\
\hline 2038 & pQlinkH Nup214 (1916-2033) & $\begin{array}{l}\text { PCR template: \#47, Primers:2054(F), 2055(R), cloned into } \\
\text { vector } \# 87\end{array}$ \\
\hline 2039 & pQlinkH Nup214 (801-950) & $\begin{array}{l}\text { PCR template: \#47, Primers:2048(F), 2049(R), cloned into } \\
\text { vector \#87 }\end{array}$ \\
\hline
\end{tabular}




\begin{tabular}{|c|c|c|}
\hline 2040 & Nup214-EGFP (L882A/L885A) & $\begin{array}{l}\text { PCR template: \#1627, Mutagenesis by primers: 1490(F), } \\
\text { 1491(R) }\end{array}$ \\
\hline 2041 & EGFP-Nup214 (L882A/L885A) & $\begin{array}{l}\text { PCR template: \#1628, Mutagenesis by primers: } 1490(\mathrm{~F}) \text {, } \\
\text { 1491(R) }\end{array}$ \\
\hline 2048 & $\begin{array}{l}\text { EGFP-Nup214 (L882A/L887A)_no } \\
\text { stop codon }\end{array}$ & $\begin{array}{l}\text { PCR template: \#2041, Primers: } 1465(\mathrm{~F}), 1466(\mathrm{R}) \text {, cloned into } \\
\text { vector \#30 }\end{array}$ \\
\hline 2064 & $\begin{array}{l}\text { pQlinkH Nup214 (801-950)-Nup214 } \\
\text { (1916-2033) }\end{array}$ & $\begin{array}{l}\text { Concatenation of pQlinlkH-Nup214(801-950) } \\
\text { (\#2039/backbone) and pQlinkH-Nup214(1916-2033) } \\
\text { (\#2038/insert) }\end{array}$ \\
\hline 2065 & $\begin{array}{l}\text { pQlinkH CRM1 . Ran (1-180)GTP- } \\
\text { Nup214 (801-950) }\end{array}$ & $\begin{array}{l}\text { Concatenation of pQlinlkH-CRM1 . Ran(1-180)GTP } \\
\text { (\#2059/backbone) and pQlinkH-Nup214(801-950) } \\
\text { (\#2039/insert) }\end{array}$ \\
\hline 2066 & $\begin{array}{l}\text { pQlinkH CRM1 . Ran (1-180)GTP- } \\
\text { Nup214 (1916-2033) }\end{array}$ & $\begin{array}{l}\text { Concatenation of pQlinlkH-CRM1 . Ran(1-180)GTP } \\
\text { (\#2059/backbone) and pQlinkH-Nup214(1916-2038) } \\
\text { (\#2038/insert) }\end{array}$ \\
\hline 2067 & $\begin{array}{l}\text { pQlinkH CRM1 . Nup214 (801-950)- } \\
\text { Nup214 (1916-2033) }\end{array}$ & $\begin{array}{l}\text { Concatenation of pQlinlkH-CRM1 (\#2043/backbone) and } \\
\text { pQlinkH-Nup214(801-950). Nup214(1916-2033) } \\
\text { (\#2064/insert) }\end{array}$ \\
\hline 2068 & $\begin{array}{l}\text { pQlinkH CRM1 . Ran (1-180)GTP- } \\
\text { Nup214 (801-950) . Nup214 (1916- } \\
\text { 2033) }\end{array}$ & $\begin{array}{l}\text { Concatenation of pQlinlkH-CRM1 . Ran(1-180)GTP } \\
\text { (\#2059/backbone) and pQlinkH-Nup214(801-950). } \\
\text { Nup214(1916-2033) (\#2064/insert) }\end{array}$ \\
\hline 2168 & $\begin{array}{l}\text { EGFP-Nup214 } \\
\text { (L882A/L887A)_Nup214 NES_Stop } \\
\text { codon }\end{array}$ & $\begin{array}{l}\text { Oligo annealing using primers } 2136(\mathrm{~F}), 2137(\mathrm{R}) \text { in plasmid } \\
\# 2048 \text { using PspOMI \& MfeI }\end{array}$ \\
\hline 2169 & $\begin{array}{l}\text { EGFP-Nup214 (L882A/L887A)_Rev } \\
\text { NES_Stop codon }\end{array}$ & $\begin{array}{l}\text { Oligo annealing using primers } 2134(\mathrm{~F}), 2135(\mathrm{R}) \text { in plasmid } \\
\text { \#2048 using PspOMI \& MfeI }\end{array}$ \\
\hline 2177 & $\begin{array}{l}\text { pEGFP-C1-Nup214 (585-1085) } \\
\text { (L882A/L887A) }\end{array}$ & $\begin{array}{l}\text { PCR template: \#1949, Mutagenesis by primers: } 1490(\mathrm{~F}) \text {, } \\
\text { 1491(R) }\end{array}$ \\
\hline 2178 & $\begin{array}{l}\text { pEGFP-C1-Nup214 (650-950) } \\
\text { (L882A/L887A) }\end{array}$ & $\begin{array}{l}\text { PCR template: \#1950, Mutagenesis by primers: 1490(F), } \\
\text { 1491(R) }\end{array}$ \\
\hline 2179 & $\begin{array}{l}\text { pEGFP-C1-Nup214 (680-1209) } \\
\text { (L882A/L887A) }\end{array}$ & $\begin{array}{l}\text { PCR template: \#1951, Mutagenesis by primers: 1490(F), } \\
\text { 1491(R) }\end{array}$ \\
\hline 2180 & $\begin{array}{l}\text { pcDNA3-Nup214 (585-1085) } \\
\text { (L882A/L887A)-HA }\end{array}$ & $\begin{array}{l}\text { PCR template: \#1955, Mutagenesis by primers: 1490(F), } \\
\text { 1491(R) }\end{array}$ \\
\hline 2181 & $\begin{array}{l}\text { pcDNA3-Nup214 (650-950) } \\
\text { (L882A/L887A)-HA }\end{array}$ & $\begin{array}{l}\text { PCR template: \#1956, Mutagenesis by primers: 1490(F), } \\
\text { 1491(R) }\end{array}$ \\
\hline 2182 & $\begin{array}{l}\text { pcDNA3-Nup214 (680-1209) } \\
\text { (L882A/L887A)-HA }\end{array}$ & $\begin{array}{l}\text { PCR template: \#1957, Mutagenesis by primers: 1490(F), } \\
\text { 1491(R) }\end{array}$ \\
\hline 2183 & pEGFP-C1-Nup214 (451-1209) & $\begin{array}{l}\text { PCR template: \#2041, Primers: } 2280(\mathrm{~F}), 1428(\mathrm{R}) \text {, cloned } \\
\text { into vector \#30 }\end{array}$ \\
\hline
\end{tabular}




\begin{tabular}{|c|c|c|}
\hline 2184 & $\begin{array}{l}\text { pEGFP-C1-Nup214 (1969-2090)_no } \\
\text { stop codon }\end{array}$ & $\begin{array}{l}\text { PCR template: \#2041, Primers: 2279(F), 1427(R), cloned } \\
\text { into vector } \# 30\end{array}$ \\
\hline 2188 & $\begin{array}{l}\text { pEGFP-C1-Nup214 (1969- } \\
\text { 2090)_Nup214 NES }\end{array}$ & $\begin{array}{l}\text { Oligo annealing using primers 2136(F), } 2137(\mathrm{R}) \text { in \#2184 } \\
\text { using PspOMI \& MfeI }\end{array}$ \\
\hline 2189 & $\begin{array}{l}\text { pEGFP-C1-Nup214 (1969- } \\
\text { 2090)_Rev NES }\end{array}$ & $\begin{array}{l}\text { Oligo annealing using primers 2134(F), } 2135 \text { (R) in \#2184 } \\
\text { using PspOMI \& MfeI }\end{array}$ \\
\hline 2190 & $\begin{array}{l}\text { pEGFP-C1-Nup214 (451-1209) } \\
\text { (L882A/L887A) }\end{array}$ & $\begin{array}{l}\text { PCR template: \#2184, Mutagenesis by primers: } 1490(\mathrm{~F}) \text {, } \\
\text { 1491(R) }\end{array}$ \\
\hline 2196 & pEGFP-Nup214 (L885A/L887A) & $\begin{array}{l}\text { PCR template: \#1628, Mutagenesis by primers: } 1490(\mathrm{~F}) \text {, } \\
\text { 1491(R) }\end{array}$ \\
\hline 2197 & $\begin{array}{l}\text { pEGFP-Nup214_siRNA resistant } \\
\text { (siNup214_4) }\end{array}$ & $\begin{array}{l}\text { PCR template: \#1628, Mutagenesis by primers: 594(F), } \\
595(\mathrm{R})\end{array}$ \\
\hline 2198 & $\begin{array}{l}\text { pEGFP-Nup214 (L885A/L887A)- } \\
\text { Nup214NES_siRNA resistant } \\
\text { (siNup214_4) }\end{array}$ & $\begin{array}{l}\text { PCR template: } \# 2168 \text {, Mutagenesis by primers: 594(F), } \\
595(\mathrm{R})\end{array}$ \\
\hline 2199 & $\begin{array}{l}\text { pEGFP-Nup214 (L885A/L887A)- } \\
\text { RevNES_siRNA resistant } \\
\text { (siNup214_4) }\end{array}$ & $\begin{array}{l}\text { PCR template: } \# 2169 \text {, Mutagenesis by primers: 594(F), } \\
\text { 595(R) }\end{array}$ \\
\hline 2200 & $\begin{array}{l}\text { pEGFP-Nup214 } \\
\text { (L885A/L887A)_siRNA resistant } \\
\text { (siNup214) }\end{array}$ & $\begin{array}{l}\text { PCR template: \#2196, Mutagenesis by primers: 594(F), } \\
\text { 595(R) }\end{array}$ \\
\hline 2204 & GFP-Nup214 (878-887) & $\begin{array}{l}\text { Oligo annealing using primers 2136(F), 2137(R) in plasmid } \\
\text { \#2048 using EcoRI and SalI }\end{array}$ \\
\hline 2205 & $\begin{array}{l}\text { GFP-Nup214 (878-887) } \\
\text { (L885A/L887A) }\end{array}$ & $\begin{array}{l}\text { Oligo annealing using primers 2136(F), 2137(R) in plasmid } \\
\# 2048 \text { using EcoRI and SalI }\end{array}$ \\
\hline
\end{tabular}




\subsection{Methods}

\subsubsection{Molecular biology based methods}

\subsubsection{Polymerase chain reaction (PCR)}

PCR reactions were set up in a total volume of $50 \mu 1$ and the reaction was run for a total of 35 cycles. Phusion High-Fidelity DNA Polymerase (ThermoFisher Scientific) was used according to the manufacturer's instructions. The melting temperature for each primer pair was calculated using the online $\mathrm{T}_{\mathrm{m}}$ calculator available from ThermoFisher Scientific and the annealing temperature was set $5^{\circ} \mathrm{C}$ below the calculated melting temperature. The elongation time was determined based on the expected PCR product size, so that 30 seconds of elongation were required per kilobase.

Following the PCR reaction, 6x DNA loading buffer was added and the resulting PCR product was loaded on an agarose gel alongside a 1 kilobase DNA marker (ThermoFisher Scientific). The percentage of the gel used was dependent on the expected PCR product size (see section 2.2.1.7). The documentation of the gel was done over the GelSTICK "touch" system (INTAS Science Imaging Instruments). Later, the DNA was visualized by an UV translaminar and the PCR product was cut out of the gel using a scalpel. The PCR product was purified using NucleoSpin ${ }^{\circledR}$ Gel and PCR Clean-up kit (Macherey-Nagel) according to the manufacturers protocol (see section 1.6.1.8). The PCR product was eluted in a total volume of $20 \mu 1$ autoclaved water.

\subsubsection{Restriction enzyme digest}

Restriction enzyme digestion reactions were set up in a total volume of $30 \mu 1$ for plasmid DNA backbones and $40 \mu \mathrm{l}$ for PCR products. The selection of the adequate buffers required for each restriction enzyme was done based on the DoubleDigest tool available online from ThermoFisher scientific (https://www.thermofisher.com/de/de/home/brands/thermoscientific/molecular-biology/thermo-scientific-restriction-modifying-enzymes/restrictionenzymes-thermo-scientific/double-digest-calculator-thermo-scientific.html).

Following the digestion reaction, 6x DNA loading buffer was added and the digestion product was loaded on an agarose gel alongside a 1 kilobase DNA marker (ThermoFisher Scientific). The percentage of the gel used depended on the expected digested product size (see section 1.6.1.7). The documentation of the gel was done using the GelSTICK "touch" system (INTAS Science Imaging Instruments). Five $\mu \mathrm{g}$ of plasmid DNA was digested for 3 hours at $37^{\circ} \mathrm{C}$ and $20 \mu \mathrm{l}$ of the eluted PCR product (see section 1.6.1.2) was digested for 2 hours at $37^{\circ} \mathrm{C}$. The digested product was run on an agarose gel as previously described. The DNA was visualized 
by an UV transilluminator and the digestion product was cut out of the gel using a scalpel. The digestion product was next purified using NucleoSpin ${ }^{\circledR}$ Gel and PCR Clean-up kit (MachereyNagel) according to the manufacturers protocol (see section 1.6.1.8). The digestion product was eluted in a total volume of $20 \mu 1$ autoclaved water.

For DNA isolated on a small scale (see section 1.6.1.2), digestion was performed for 1 hour at $37^{\circ} \mathrm{C}$ using $5 \mu \mathrm{l}$ of plasmid DNA. The digested product was run on an agarose gel and later visualized as described above.

\subsubsection{Oligonucleotide-directed mutagenesis}

Phusion High-Fidelity DNA Polymerase (ThermoFisher Scientific) was used to set up sitedirect mutagenesis reactions. Forward and reverse oligonucleotides containing the mutation sites were synthesized by Sigma-Aldrich (Merck). The mutagenesis reactions were assembled in a total volume of $50 \mu \mathrm{l}$ and the PCR reaction was run for a total of 17 cycles. The annealing temperature was set at a universal temperature of $60^{\circ} \mathrm{C}$ for all primer pairs and the elongation time was determined based on the expected PCR product size so that 30 seconds of elongation was required for each 1 kilobase.

Following the PCR reaction, $1 \mu$ of DpnI (10 U, ThermoFisher Scientific) was added to each PCR mixture to degrade the template and the mixture was then incubated for 2 hours at $37^{\circ} \mathrm{C}$. Finally, $7 \mu \mathrm{l}$ of the PCR reaction was transformed in DH5 $\alpha$ using a standard transformation protocol (see section 1.6.1.9).

\subsubsection{Annealing of oligonucleotides}

Oligonucleotides were designed with 3'and 5'sticky ends according to the desired restriction sites. Oligonucleotide annealing reactions were set up by mixing $2 \mu \mathrm{g}$ of the sense and antisense strands in annealing buffer $(30 \mathrm{mM}$ Hepes $\mathrm{KOH} \mathrm{pH} 7.4,100 \mathrm{mM}$ KOAc, $2 \mathrm{mM}$ $\left.\mathrm{Mg}(\mathrm{OAc})_{2}\right)$. The reaction mixture was first incubated at $90^{\circ} \mathrm{C}$ for 3 minutes and subsequently incubated at $37^{\circ} \mathrm{C}$ for 1 hour. Following the incubation time, $10 \mu \mathrm{l}$ of the annealed oligonucleotides were phosphorylated using $1 \mu \mathrm{l}$ of T4-Polynucleotidkinase (EK0031, $10 \mathrm{U} / \mathrm{L}$, ThermoFisher Scientific), $2 \mu 1$ of 10x buffer PNK A (Fermentas) and $2 \mu 1$ of $10 \mathrm{mM}$ ATP (ThermoFisher) in a total volume of $20 \mu \mathrm{l}$ water. Next the phosphorylation mix was incubated at $37^{\circ} \mathrm{C}$ for 20 minutes and subsequently at $75^{\circ} \mathrm{C}$ for 10 minutes followed by inactivation on ice. 


\subsubsection{Quantification of DNA}

NanoDrop 2000c (ThermoScientific) was used to determine the concentration and purity of dsDNA by measuring the UV absorbance at $260 \mathrm{~nm}$ and $280 \mathrm{~nm}$. All measurements were blanked against water.

\subsubsection{DNA ligation}

Ligation reactions were set up in volumes of $10 \mu \mathrm{l}$ using $1 \mu \mathrm{g} / \mu \mathrm{l}$ ATP (ThermoFisher Scientific), 1x T4 DNA Ligase Reaction Buffer (ThermoFisher Scientific) and $0.5 \mu$ of T4 DNA Ligase (ThermoFisher Scientific). $100 \mathrm{ng}$ of the vector was ligated with $3 \mathrm{x}$ or 5x molar excess of the insert DNA fragment. The reaction mixture was incubated at room temperature for 2 hours and subsequently transformed into chemically competent DH5 $\alpha$ cells using a standard transformation protocol (see section 1.6.1.9).

\subsubsection{Agarose gel electrophoresis}

Agarose gels were casted in percentages of $0.5 \%-2 \%$ according to the expected size of the DNA fragments and the resolution required. The gel preparation was done by dissolving the agarose in 1x TAE buffer ( $40 \mathrm{mM}$ Tris, $1 \mathrm{mM}$ EDTA, $0.114 \%$ acetic acid) in a glass bottle and boiling it in the microwave until completely dissolved. The agarose was left to cool down and then $1 \mu$ l of SERVA DNA stain clear G (SERVA) was added per $10 \mathrm{ml}$ of the agarose solution, followed by casting the gel. The agarose gel electrophoresis run was done in 1x TAE buffer. The current required varied from $80 \mathrm{~V}$ to $120 \mathrm{~V}$ and the time required varied from 30 minutes to 60 minutes depending on the size and the percentage of the agarose gel used.

\subsubsection{PCR reaction Clean-up and plasmid DNA purification}

Purification of PCR reactions and plasmid DNA was done using NucleoSpin ${ }^{\circledR}$ Plasmid kit (Macherey-Nagel) according to the manufacturer's instructions

For small scale DNA isolation, $3 \mathrm{ml}$ of LB $(1 \%(\mathrm{w} / \mathrm{v})$ bacto-tryptone, $0.5 \%(\mathrm{w} / \mathrm{v})$ yeast extract, $1 \%(\mathrm{w} / \mathrm{v}) \mathrm{NaCl}(\mathrm{pH} 7.0))$ supplemented with the appropriate antibiotic was inoculated with one colony picked from an LB-agar plate. Cultures were incubated overnight at $37{ }^{\circ} \mathrm{C}, 180 \mathrm{rpm} .2$ $\mathrm{ml}$ of culture was transferred to a $2 \mathrm{ml}$ reaction tube and cells were harvested by centrifugation (11000 g, $4{ }^{\circ} \mathrm{C}, 1$ minute). DNA was extracted with the NucleoSpin ${ }^{\circledR}$ Plasmid kit (MachereyNagel) according to the manufacturer's instructions. The purified DNA was eluted with $50 \mu 1$ of elution buffer. 
For large scale plasmid DNA isolation, the remaining $1 \mathrm{ml}$ from the mini prep or a single picked colony was used to inoculate $200 \mathrm{ml}$ of LB media supplemented with the suitable antibiotic. The cultures were incubated overnight at $37^{\circ} \mathrm{C}$ while shaking at $150 \mathrm{rpm}$.

The cells were harvested by centrifugation and DNA was extracted with the NucleoSpin ${ }^{\circledR}$ Plasmid (Macherey-Nagel) according to the manufacturer's instructions. The DNA was precipitated using $3.5 \mathrm{ml}$ isopropanol. The DNA precipitate was dissolved in $300 \mu \mathrm{l}$ of autoclaved water and the final concentration of the DNA was adjusted to $1 \mathrm{mg} / \mathrm{ml}$.

\subsubsection{Transformation of bacteria}

Transformation of $E$. coli DH5 $\alpha$ competent cells was done in case of re-transformation of a plasmid DNA, transformation of a ligation reaction and transformation of an oligonucleotidedirected mutagenesis reaction.

In case of bacterial protein expression, other competent bacterial cells were transformed according to the protein intended for expression (see section 1.6.2.1).

First, a $100 \mu \mathrm{l}$ aliquot of DH5 $\alpha$ competent cells were thawed on ice. Next, $0.2 \mu \mathrm{g}$ of plasmid DNA or $10 \mu \mathrm{l}$ of a ligation reaction or $7 \mu \mathrm{l}$ of an oligonucleotide-directed mutagenesis reaction was added to the thawed bacterial cells. The cells were mixed thoroughly and then incubated on ice for 20 minutes. Following this incubation, the bacteria were heat shocked at $42^{\circ} \mathrm{C}$ for 90 seconds and subsequently cooled down on ice for 5 minutes. After that, $300 \mu \mathrm{l}$ of ice-cold SOC medium $(2 \%(\mathrm{w} / \mathrm{v})$ tryptone, $0.5 \%(\mathrm{w} / \mathrm{v})$ yeast extract, $10 \mathrm{mM} \mathrm{NaCl}, 2.5 \mathrm{mM} \mathrm{KaCl}, 10 \mathrm{mM}$ $\mathrm{MgCl}_{2}, 10 \mathrm{mM} \mathrm{MgSO} 4,0.36 \%$ (w/v) glucose, $\mathrm{pH}$ 7.0) were added and the mixture was incubated in a ThermoMixer (Eppendorf) at $37^{\circ} \mathrm{C}$ while shaking at $650 \mathrm{rpm}$ for 90 minutes. Finally, $200 \mu \mathrm{l}$ of the mixture (or $50 \mu \mathrm{l}$ in case of re-transformations) were plated using glass beads on an LB-agar plate (1.5\% (w/v) bacto-agar dissolved in LB media) supplemented with the appropriate antibiotics. The plates were then incubated overnight at $37^{\circ} \mathrm{C}$.

\subsubsection{DNA sequencing}

30-100 ng/ $\mu 1$ of plasmid DNA in a total volume of $20 \mu \mathrm{l}$ was sent for sequencing at Eurofins genomics using either standard primers provided by the company or a custom primer targeting a region of interest. For custom primers, $20 \mu \mathrm{l}$ of $10 \mathrm{uM}$ of the primer was sent with the sample. Analysis of the resulting sequence was done using SeqBuilder software (Lasergene). 


\subsubsection{Biochemistry based methods}

\subsubsection{Purification of proteins}

CRM1-GST, GST-Nup214 (878-887), GST-Nup214 (878-887)_L878A/L882A, His-Nup214 (1916-2033), His-SPN1, MBP-Nup214 (1916-2033), Nup214 (1916-2033), RanBP1 and RanGAP were available in our common stock.

All proteins were centrifuged for 10 minutes at $10000 \mathrm{rpm}$ at $4^{\circ} \mathrm{C}$ prior to use to remove any possible precipitates. The concentration of these proteins was double checked using Bradford assay. The molar concentration of the proteins was calculated using Bioline molar calculator tool that is available online (http://www.bioline.com/us/media/calculator/01_04.html).

\subsection{Purification of $\operatorname{Ran}_{\mathrm{Q} 69 \mathrm{~L}(1-180) \mathrm{GTP}}$}

On day one, $0.2 \mu \mathrm{g}$ of pQE80-His $10 \mathrm{ZZ}-\mathrm{Ran}_{\mathrm{Q} 69 \mathrm{~L}(1-180)}$ was transformed in E. coli BL21 (DE3) pLysS bacteria using standard transformation protocol (see section 1.6.1.9) and the bacteria were plated on LB plates supplemented with $100 \mathrm{mg} / \mathrm{ml}$ ampicillin and $30 \mathrm{mg} / \mathrm{ml}$ chloramphenicol, then incubated overnight at $37^{\circ} \mathrm{C}$. Next morning a pre-culture was prepared; $7 \mathrm{ml}$ of 2YT medium supplemented with $100 \mathrm{mg} / \mathrm{ml}$ ampicillin and $30 \mathrm{mg} / \mathrm{ml}$ chloramphenicol were inoculated with 3 to 6 colonies and incubated overnight at $37^{\circ} \mathrm{C}$. Flasks containing $900 \mathrm{ml}$ of 2YT medium supplemented with $100 \mathrm{mg} / \mathrm{ml}$ ampicillin, $30 \mathrm{mg} / \mathrm{ml}$ chloramphenicol, $20 \mathrm{~g}$ glucose monohydrate were inoculated with $10 \mathrm{ml}$ of the overnight pre-culture. All cultures were incubated at $37^{\circ} \mathrm{C}$ while shaking at a speed of $220 \mathrm{rpm}$. The $\mathrm{OD}_{600}$ was measured every 30 minutes and a stepwise decrease of temperature was done according to the measured $\mathrm{OD}_{600}$. First, the flasks were incubated at $30^{\circ} \mathrm{C}$ once the $\mathrm{OD}_{600}$ reached $0.35-0.4$. Then, at an $\mathrm{OD}_{600}$ of 0.5 , the flasks were incubated at $23^{\circ} \mathrm{C}$. Next, they were moved to an $18^{\circ} \mathrm{C}$ incubator at an $\mathrm{OD}_{600}$ of 0.7. Finally, the bacterial expression was induced at $18^{\circ} \mathrm{C}$ using $0.5 \mathrm{uM} \mathrm{IPTG}$ at an $\mathrm{OD}_{600}$ of 1.0 and the cultures were incubated overnight. The following day, bacterial cells were harvested at an $\mathrm{OD}_{600}$ of 3-4 using an Allegra ${ }^{\circledR} \mathrm{X}-15 \mathrm{R}$ (Beckman Coulter) table top centrifuge at a speed of $4800 \mathrm{rpm}$ rotor for 20 minutes at $4^{\circ} \mathrm{C}$. The resulting pellets were re-suspended thoroughly in $10 \mathrm{ml}$ HisTrap lysis buffer (50 mM Hepes, $\mathrm{pH}$ 7.5, $500 \mathrm{mM} \mathrm{NaCl}, 3 \mathrm{mM}$ imidazole, $5 \mathrm{mM}$ $\mathrm{MgCl}_{2}, 10 \%$ glycerol, $1 \mathrm{mM}$ DTT, $30 \mu \mathrm{M}$ GTP, 1 Roche protease inhibitor tablet, $100 \mathrm{mg} / \mathrm{ml}$ DNase I) and the cells were lysed by passing it seven times though an EmulsiFlex-C3 emulsifier (Avestin). The lysate was then pelleted using an AvantiTM J-30I ultracentrifuge (Beckman Coulter) at a speed of $20,000 \mathrm{rpm}$ for 45 minutes and a temperature of $4^{\circ} \mathrm{C}$. The pellet was discarded and the resulting supernatant was prepared for injection into an affinity exchange 
chromatography column by filtering it two times through an 0.2 um filter and then loading it on a $150 \mathrm{ml}$ super-loop for injection.

First, the supernatant underwent a first step of affinity purification using four ÄKTA His Trap FF $5 \mathrm{ml}$ columns (GE Healthcare) connected to an ÄKTApurifier HPLC (Amersham Biosciences). In detail, the column was first equilibrated in HisTrap buffer A (50 mM Hepes pH 7.5, $500 \mathrm{mM} \mathrm{NaCl}, 3 \mathrm{mM}$ imidazole, $5 \mathrm{mM} \mathrm{MgCl}_{2}, 10 \%$ glycerol, $1 \mathrm{mM} \mathrm{DTT,} 30 \mu \mathrm{M}$ GTP in water) and the supernatant was injected into the column. Then, the column was washed with HisTrap buffer A containing low concentration of imidazole to remove the unspecific proteins binding to the column. Next, the bound $\mathrm{His}_{10} \mathrm{ZZ}-\mathrm{Ran}_{\mathrm{Q} 69 \mathrm{~L}(1-180)}$ was eluted with an increasing concentration of HisTrap buffer B (50 mM Hepes pH 7.5, $500 \mathrm{mM} \mathrm{NaCl}, 500 \mathrm{mM}$ imidazole, $5 \mathrm{mM} \mathrm{MgCl}_{2}, 10 \%$ glycerol, $1 \mathrm{mM} \mathrm{DTT}, 30 \mu \mathrm{M} \mathrm{GTP}$ ) at a flow rate of $1 \mathrm{ml} / \mathrm{min}$ and the protein containing fractions were pooled in a beaker.

Second, the protein was desalted to remove the imidazole. In detail, an ÄKTA HiPrep 26/10 desalting column (Sephadex G-25, GE Healthcare) was equilibrated in HisTrap desalting buffer (50 mM Hepes pH 7.5, $500 \mathrm{mM} \mathrm{NaCl}, 3 \mathrm{mM}$ imidazole, $5 \mathrm{mM} \mathrm{MgCl} 2,10 \%$ glycerol, $1 \mathrm{mM}$ DTT, $30 \mu \mathrm{M}$ GTP, 1 Roche protease inhibitor tablet, $100 \mathrm{mg} / \mathrm{ml}$ DNase I in water) and the eluate was loaded onto the desalting column at a flow rate of $5-10 \mathrm{ml} / \mathrm{min}$. The protein containing fractions were pooled in a beaker. Finally, the total protein concentration was measured using a standard Bradford assay.

Third, the $\mathrm{His}_{10} \mathrm{ZZ}-\mathrm{Ran}_{\mathrm{Q} 69 \mathrm{~L}(1-180)}$ was digested using $1.5 \mathrm{mg}$ of TEV protease for each $100 \mathrm{mg}$ of the protein. An overnight digestion was performed on a rotor at $4{ }^{\circ} \mathrm{C}$.

Fourth, the untagged $\operatorname{Ran}_{\mathrm{Q} 69 \mathrm{~L}(1-180)}$ resulting from the TEV protease digestion was collected in the flow through by running the protein over four ÄKTA His Trap FF $5 \mathrm{ml}$ columns. The sample was prepared by filtering it two times through an $0.2 \mathrm{um}$ filter and loading it on a $150 \mathrm{ml}$ superloop. In detail, the columns were first equilibrated in desalting buffer and the sample was injected. Next, the fractions containing the $\mathrm{Ran}_{\mathrm{Q} 69 \mathrm{~L}(1-180)}$ that has been cleaved by TEV protease were pooled and checked by SDS page (see section 1.6.2.6). Finally, the pooled fractions were concentrated to $4 \mathrm{ml}$ by centrifugation at $4000 \mathrm{~g}$ using a $6 \mathrm{ml}$ Corning Spin-X® UF concentrator (Merk) with MWCO of 10.000 Daltons.

Fifth, a final purification step was performed using size exclusion chromatography. In detail, an ÄKTA HiLoad 26/60 Superdex 200 prep grade (S200 XK26/60, GE Healthcare) column was equilibrated in desalting buffer and the concentrated protein was loaded onto the column at a flow rate of $1 \mathrm{ml} / \mathrm{min}$. The $\mathrm{Ran}_{\mathrm{Q} 69 \mathrm{~L}(1-180)}$ containing fractions were pooled and checked by SDS page (see section 1.6.2.6). 
Finally, the pooled fraction from the final purification step were concentrated to $5 \mathrm{mg} / \mathrm{ml}$ by centrifugation at $4000 \mathrm{~g}$ using a $6 \mathrm{ml}$ Corning Spin-X® UF concentrator (Merk) with MWCO of 10.000 Daltons. The resulting protein was frozen at $-80^{\circ} \mathrm{C}$ in aliquots of $30-50 \mathrm{ul}$.

\subsection{Purification of CRM1-His}

On day one, T7-CRM1-His pET21a was transformed in E. coli BL21 (DE3) bacteria using standard transformation protocol and the bacteria were plated on LB plates supplemented with $100 \mathrm{mg} / \mathrm{ml}$ ampicillin, then incubated overnight at $37^{\circ} \mathrm{C}$. Next morning a pre-culture was prepared, where $50 \mathrm{ml}$ of $2 \mathrm{YT}$ medium supplemented with $100 \mathrm{mg} / \mathrm{ml}$ ampicillin were inoculated with 3 to 6 colonies and incubated overnight at $37^{\circ} \mathrm{C}$. Flasks containing $1000 \mathrm{ml}$ of 2YT medium supplemented with $100 \mathrm{mg} / \mathrm{ml}$ ampicillin were inoculated with $10 \mathrm{ml}$ of the overnight pre-culture. Cultures were incubated at $37^{\circ} \mathrm{C}$ to an $\mathrm{OD}_{600}$ of 0.3 while shaking at a speed of $220 \mathrm{rpm}$, then transferred to an $18{ }^{\circ} \mathrm{C}$ incubator. The $\mathrm{OD}_{600}$ was measured every 30 minutes and the bacterial expression was induced using $0.1 \mathrm{uM} \mathrm{IPTG}$ once the $\mathrm{OD}_{600}$ reached 0.6. The cultures were then incubated overnight at $18^{\circ} \mathrm{C}$ and the bacteria were harvested next morning at an $\mathrm{OD}_{600}$ of 3-4 using an Allegra ${ }^{\circledR}$ X-15R (Beckman Coulter) at $4800 \mathrm{rpm}$ for 20 minutes at $4^{\circ} \mathrm{C}$. In all previous incubation steps, the cultures were shaken at $220 \mathrm{rpm}$. The resulting pellets were re-suspended thoroughly in $50 \mathrm{ml}$ HisTrap buffer A (50 mM HEPES pH 7.5, $500 \mathrm{mM} \mathrm{NaCl}, 30 \mathrm{mM}$ imidazole, $2 \mathrm{mM} \mathrm{MgCl} 2,10 \%$ glycerol, $4 \mathrm{mM}$ ß-mercaptoethanol) supplemented with 1 protease inhibitor cOmplete ULTRA tablet (Roche) and the cells were lysed by passing it five times though an EmulsiFlex-C3 emulsifier (Avestin). The lysate was cleared by centrifugation at $20000 \mathrm{rpm}$ and at $4{ }^{\circ} \mathrm{C}$ for 45 minutes using an AvantiTM J-30I ultracentrifuge (Beckman Coulter) at a speed of $50000 \mathrm{rpm}$ for 45 minutes and a temperature of $4^{\circ} \mathrm{C}$. The cleared lysate was loaded on $3 \times 5 \mathrm{ml}$ HisTrap columns pre-equilibrated in CRM1 HisTrap buffer A and eluted in CRM1 HisTrap buffer B (50 mM HEPES pH 7.5, $500 \mathrm{mM}$ $\mathrm{NaCl}, 400 \mathrm{mM}$ imidazole, $4 \mathrm{mM} \mathrm{MgCl} 2,3 \mathrm{mM}$ 2-mercaptoethanol. Fractions containing CRM1 were pooled and dialyzed using CRM1 dialysis buffer (50 mM HEPES pH7.5, $50 \mathrm{mM} \mathrm{NaCl}, 2$ $\mathrm{mM} \mathrm{MgCl} 2,5 \%$ Glycerol, $6 \mathrm{mM}$ 2-mercaptoethanol). Following dialysis, anion exchange chromatography was performed using a mono Q column. The column was equilibrated in mono Q buffer A (50 mM HEPES pH 7.8, $50 \mathrm{mM} \mathrm{NaCl}, 2 \mathrm{mM} \mathrm{MgCl}_{2}, 6 \mathrm{mM}$ 2-mercaptoethanol) and the elution was done with mono Q buffer B (50 mM HEPES pH 7.8, $400 \mathrm{mM} \mathrm{NaCl}, 2 \mathrm{mM}$ $\mathrm{MgCl}_{2}, 6 \mathrm{mM}$ 2-mercaptoethanol) with a gradient of $0-70 \%$ over $300 \mathrm{ml}$. The CRM1-containing fractions were pooled and concentrated using Vivaspin ${ }^{\circledR} 20$ Ultrafiltration Unit (Sartorius) with a MWCO of $30 \mathrm{kDa}$. 


\subsection{Purification of Nup214 fragments}

\section{- MBP-tagged Nup214 fragments}

On day one, MBP-Nup214-His was transformed in E. coli BL21 (DE3) codon+ bacteria using standard transformation protocol (see section 1.6.1.9) and the bacteria were plated on LB plates supplemented with $100 \mathrm{mg} / \mathrm{ml}$ ampicillin and $30 \mathrm{mg} / \mathrm{ml}$ chloramphenicol, then incubated overnight at $37^{\circ} \mathrm{C}$. Next morning a pre-culture was prepared; $100 \mathrm{ml}$ of 2 YT medium supplemented with $100 \mathrm{mg} / \mathrm{ml}$ ampicillin and $30 \mathrm{mg} / \mathrm{ml}$ chloramphenicol were inoculated with 3 to 6 colonies and incubated overnight at $37^{\circ} \mathrm{C}$. Flasks containing $1000 \mathrm{ml}$ of 2 YT medium supplemented with $100 \mathrm{mg} / \mathrm{ml}$ ampicillin and $30 \mathrm{mg} / \mathrm{ml}$ chloramphenicol were inoculated with $10 \mathrm{ml}$ of the overnight pre-culture. Bacterial cultures were incubated at $37^{\circ} \mathrm{C}$ and grown to an $\mathrm{OD}_{600}$ of 0.6 while shaking at a speed of $150 \mathrm{rpm}$. The bacterial cultures were cooled down to $18^{\circ} \mathrm{C}$ and transferred to an $18{ }^{\circ} \mathrm{C}$ incubator. The protein expression was induced overnight at $18^{\circ} \mathrm{C}$ using $0.2 \mathrm{uM}$ IPTG while shaking at $100 \mathrm{rpm}$. Bacteria were harvested at an $\mathrm{OD}_{600}$ of 12 using an Allegra ${ }^{\circledR}$ X-15R (Beckman Coulter) at a speed of $4800 \mathrm{rpm}$ for 20 minutes at $4^{\circ} \mathrm{C}$. The resulting pellets were re-suspended thoroughly in $15 \mathrm{ml}$ MBP-lysis buffer (20 mM Hepes, pH 8.0, $100 \mathrm{mM} \mathrm{KCl}, 10 \mathrm{mM} \mathrm{Mg}(\mathrm{OAc})_{2}, 10 \%$ glycerol, $0.01 \%$ TritonX-100, $1 \mathrm{mM}$ DTT, 1 $\mu \mathrm{g} / \mu \mathrm{l}$ AP, $1 \mu \mathrm{g} / \mu \mathrm{LP}, 1 \mathrm{mM}$ DTT, $1 \mathrm{mM}$ PMSF) and the cells were lysed by passing them five times though an EmulsiFlex-C3 emulsifier (Avestin). The lysate was then pelleted using an AvantiTM J-30I ultracentrifuge (Beckman Coulter) at a speed of 50,000 rpm for 45 minutes and a temperature of $4^{\circ} \mathrm{C}$. The pellet was discarded and the resulting supernatant was prepared for affinity purification.

$1.5 \mathrm{ml} \mathrm{Ni-NTA} \mathrm{Agarose} \mathrm{(QIAGEN)} \mathrm{for} \mathrm{each} \mathrm{liter} \mathrm{of} \mathrm{bacterial} \mathrm{culture} \mathrm{was} \mathrm{equilibrated} \mathrm{by}$ washing three times in MBP-binding buffer (20 mM Hepes, pH 8.0, $100 \mathrm{mM} \mathrm{KCl,} 10 \mathrm{mM}$ $\mathrm{Mg}(\mathrm{OAc})_{2}, 10 \%$ glycerol, $0.01 \%$ TritonX-100, $1 \mathrm{mM}$ DTT $)$. Then, the clear lysate was incubated with the equilibrated beads on a rotor for 1 hour at $4^{\circ} \mathrm{C}$. Next, the beads were washed three times with MBP-binding buffer supplemented with $3 \mathrm{mM}$ imidazole to remove all weekly bound unspecific proteins. After that, the MBP-Nup214-His was eluted in MBP-binding buffer supplemented with $500 \mathrm{mM}$ imidazole. The fractions containing the MBP-Nup214 fragments were pooled and the protein concentration was measured using a standard Bradford assay. The protein was further purified using amylose resin (\#E8021S, New England Biolabs), where $1 \mathrm{ml}$ of amylose resin for each 40 grams of protein was equilibrated by washing three times in MBP-binding buffer. The equilibrated beads were then incubated with the protein on a rotor for 1 hour at $4^{\circ} \mathrm{C}$. Next, the beads were washed three times with MBP-binding buffer and the protein was subsequently eluted in MBP-binding buffer supplemented with $15 \mathrm{mM}$ maltose. 
The protein-containing fractions were pooled and dialyzed against MBP-binding buffer overnight. In brief, the dialysis was performed using a slide-A-lyzer (TermoFisher Scientific) with two times buffer exchanges for two hours, followed by overnight dialysis against the MBPbinding buffer. Finally, the dialyzed protein was concentrated to $1 \mathrm{mg} / \mathrm{ml}$ using a $6 \mathrm{ml}$ Corning Spin-X® UF concentrator (Merk) with a MWCO of $20 \mathrm{kDa}$. The resulting protein was frozen at $-80^{\circ} \mathrm{C}$ in aliquots of $30-50 \mathrm{ul}$.

\section{- GST-tagged Nup214 fragments}

The plasmids coding for the GST-Nup214-His fragments were transformed in E. coli BL21 (DE3) codon+ bacteria. The purification steps were done following the same procedure described above. The lysis of the harvested bacteria was done in GST-lysis buffer (50 mM Tris $\mathrm{HCl} \mathrm{pH}$ 6.8, $150 \mathrm{mM} \mathrm{NaCl}, 1 \mathrm{mM} \mathrm{MgCl} 2,1 \mathrm{mM}$ EDTA, 0.1\% Trition X-100, 25 mM Glucose, $1 \mathrm{mM}$ DTT, $1 \mu \mathrm{g} / \mathrm{ml} \mathrm{AP,} 1 \mu \mathrm{g} / \mathrm{ml} \mathrm{LP})$. The lysed cells were centrifuged and the clear lysate was added to Glutathione Sepharose 4 fast flow beads. The immobilized GST-tagged proteins were eluted in GST-elution buffer $(50 \mathrm{mM}$ Tris $\mathrm{HCl} \mathrm{pH} 8.0,300 \mathrm{mM} \mathrm{NaCl}, 1 \mathrm{mM} \mathrm{MgCl}, 15 \mathrm{mM}$ Glutathione, $1 \mathrm{mM}$ DTT, $1 \mu \mathrm{g} / \mathrm{ml} \mathrm{AP,} 1 \mu \mathrm{g} / \mathrm{ml} \mathrm{LP}$ ). The collected protein was concentrated and the protein was dialysed against GST-dialysis buffer $(50 \mathrm{mM}$ Tris $\mathrm{HCl} \mathrm{pH} 6.8,300 \mathrm{mM} \mathrm{NaCl}$, $1 \mathrm{mM} \mathrm{MgCl} 2,0.25$ mM EDTA, 1 mM DTT, $1 \mu \mathrm{g} / \mathrm{ml} \mathrm{AP,} 1 \mu \mathrm{g} / \mathrm{ml} \mathrm{LP})$

\subsubsection{Generation of Primary Antibodies}

\subsection{Affinity purification of antibodies against CRM1}

The CRM1 C-terminal peptide (GIFNPHEIPEEMCDKLH) was used for injection in goat to generate anti-CRM1 antibodies and serum was obtained and frozen in aliquots (performed by Stefanie Roloff). It was then used for purification of antibodies. On day one, 1.2 mg of CRM1His was dialyzed against $0.2 \mathrm{M} \mathrm{Na}_{2} \mathrm{CO}_{3} \mathrm{pH} 8.9$ by performing a two-time buffer change each for two hours followed by an overnight dialysis at $4^{0} \mathrm{C}$. On day two, $0.7 \mathrm{~g}$ of $\mathrm{CNBr}$ beads (cyanogen bromide activated sepharose 4B; Sigma-Aldrich) were first left to swell in $1 \mathrm{mM}$ cold $\mathrm{HCl}$ for 30 to 60 minutes by performing five time changes with $50 \mathrm{~mL}$ each and recovering the beads by centrifugation after every change for 2 minutes at $200 \mathrm{~g}$. Then, the beads were washed two times in water and recovered by centrifugation after every wash for 2 minutes at $200 \mathrm{~g}$. Next, the beads were washed briefly in $15 \mathrm{ml}$ of $0.2 \mathrm{M} \mathrm{Na}_{2} \mathrm{CO}_{3} \mathrm{pH} 8.9$ and added immediately to the dialyzed CRM1-His in a total volume of $15 \mathrm{ml}$. The bead-protein mix was then incubated on a rotor for 1 hour at room temperature and subsequently overnight at $4^{0} \mathrm{C}$. On the third day, the beads were recovered by centrifugation as described above and the supernatant was discarded. The beads were then washed two times using $50 \mathrm{ml} 0.2 \mathrm{M} \mathrm{Na}_{2} \mathrm{CO}_{3}$ 
pH 8.9. Next, $100 \mathrm{mM}$ ethanolamine diluted in $0.2 \mathrm{M} \mathrm{Na}_{2} \mathrm{CO}_{3} \mathrm{pH} 8.9$ was used to block the remaining reactive amide groups by incubating for 1 hour at room temperature on a rotor. Next, the beads were washed 3 times with $50 \mathrm{ml}$ of $0.2 \mathrm{M} \mathrm{Na}_{2} \mathrm{CO}_{3} \mathrm{pH} 8.9$ and then once using $0.5 \mathrm{M}$ $\mathrm{NaCl}$ diluted in PBS (137 mM NaCl, pH 7.5, $2.7 \mathrm{mM} \mathrm{KCl}, 10 \mathrm{mM} \mathrm{Na}_{2} \mathrm{HPO}_{4}, 1.8 \mathrm{mM} \mathrm{KH}_{2} \mathrm{PO}_{4}$ ). The serum was diluted at a 1:1 ratio in PBS, followed by filtration through a 0.2 um filter. The serum was then added to the beads and the falcon tube was incubated overnight on a rotor at $4^{\circ} \mathrm{C}$.

On day four, the bead-serum mixture was transferred to a plastic column where it was washed with 100 column volumes of $0.5 \mathrm{M} \mathrm{NaCl}$ diluted in PBS and subsequently bound antibodies were eluted with $0.2 \mathrm{M}$ acetic acid diluted in $0.5 \mathrm{M} \mathrm{NaCl}, \mathrm{pH} 2.5 .500 \mu$ l fractions were collected in $1.5 \mathrm{ml}$ Eppendorf tubes and neutralized immediately with $100 \mathrm{uM}$ Tris $\mathrm{HCl}, \mathrm{pH}$ 6.8. The antibody containing fractions were determined using Ponceau red staining solution and pooled. The antibody was concentrated using Vivaspin® 20 Ultrafiltration Unit (Sartorius) with a MWCO of $30 \mathrm{kDa}$ and buffer exchange was done using sterile PBS. The antibodies were stored at $-20^{\circ} \mathrm{C}$ in $50 \%$ glycerol and the appropriate working concentration was determined by western blotting (see section 1.6.3.6) and immunofluorescence (see section 1.6.3.5).

\subsection{Affinity purification of antibodies against Nup214}

Purification of antibodies against Nup214 was performed using the previously described procedure for purification of anti-CRM1 antibodies. To generate Nup214 antibodies, purified Nup2 14 (1916-2033) protein was sent for injection in rabbits (Davids Biotechnologie GmbH, Germany) and the collected serum was used to extract the generated antibodies.

\subsubsection{Fluorescence Polarization Assay}

Three fluorescently tagged NES peptides of Nup214, PKI and HIV-Rev were synthesized by EMC microcollections (Tübingen, Germany). A 6-Carboxyfluorescein (6-FAM) was attached to a lysine residue in the NES peptide sequence. The fluorescent peptides were used to determine the binding constant $\left(\mathrm{K}_{\mathrm{d}}\right)$ of these NESs to CRM1. A fixed concentration of the NES peptide $(40 \mathrm{nM})$ and $\operatorname{Ran}_{\mathrm{Q} 69 \mathrm{~L}}(1-180) \mathrm{GTP}(3 \mathrm{uM})$ was titrated with increasing amounts of CRM1 (0.5 $\mathrm{nM}$ to $2000 \mathrm{nM})$ at $25^{\circ} \mathrm{C}$ in a final volume of $150 \mu \mathrm{L}$ of anisotropy assay buffer $(20 \mathrm{mM}$ Tris $\mathrm{HCl}, \mathrm{pH} 7.4,130 \mathrm{mM} \mathrm{NaCl}, 2 \mathrm{mM} \mathrm{Mg}(\mathrm{OAc})_{2}$ supplemented with $1 \mathrm{mM}$ DTT and $0.005 \%$ digitonin). Fluorescence polarization was measured using a FluoroMax 4 fluorometer (Horiba). The $G$ factor of the machine was determined by exciting the sample using both vertically (V) and horizontally $(\mathrm{H})$ polarized light for the same period of time and successively measuring the 
vertical and horizontal polarized components of the emission intensity (VV, VH and $\mathrm{HH}, \mathrm{HV}$, respectively). Excitation and emission wavelengths were set to 470 and $520 \mathrm{~nm}$, respectively. The buffer, fluorescently tagged NES peptide (fNES), Ran ${ }_{\mathrm{Q} 69 \mathrm{~L}(1-180) \mathrm{GTP}}$ and CRM1 were added in that order. All samples were incubated at $25^{\circ} \mathrm{C}$ for 30 minutes prior to the measurement. The integration time and slit width were set at the default values of the machine and the gain was automatically adjusted. As a negative control, the same experiment was repeated in the absence of $\operatorname{Ran}_{\mathrm{Q} 69 \mathrm{~L}(1-180)} \mathrm{GTP}$.

Fluorescence anisotropy competition experiments were performed by forming a complex in the presence of $100 \mathrm{nM}$ of CRM1, followed by titration of the formed complex against increasing concentrations of the competitors; GST-Nup214 (878-887) and GST-Nup214 (878887)_L878/L882A at $25^{\circ} \mathrm{C}$. Measurements were conducted using the same parameters described above. Fluorescence anisotropy values (arbitrary units) were plotted and fitted using FluorEssence software (Horiba) against increasing concentrations of CRM1 (nM) or the competitor (nM).

\subsubsection{Gel Filtration of pre-formed Nup214 export complex}

An export complex of CRM1 and Nup214 complex was formed and its integrity was tested by gel filtration chromatography. A standard export complex would contain CRM1, RanGTP, Nup214 FG-repeat fragment and the Nup214 NES. His-tagged CRM1, Ran ${ }_{Q 69 L}(1-180)$ GTP, Nup214 (1916-2033) and Nup214 fluorescently tagged NES peptide (fNup214NES) were used. Different experimental set ups have been used including formation of the full complex using all four components or formation of the complex while missing either $\operatorname{Ran}_{\mathrm{Q} 69 \mathrm{~L}(1-180) \mathrm{GTP}}$ or Nup214 (1916-2033). All components were used at an equimolar ratio of $1 \mathrm{nmol}$ per each component, except for $\mathrm{Ran}_{\mathrm{Q} 69 \mathrm{~L}(1-180) \mathrm{GTP}}$, which was added in 3-fold excess. The components of each experimental set up were added in a total volume of $150 \mu 1$ export complex buffer (20 mM Tris HCl, pH 7.4, $150 \mathrm{mM}$ Nacl, $2 \mathrm{mM} \mathrm{Mg(OAc)})_{2}$ supplemented with $1 \mathrm{mM}$ DTT) and incubated on ice for 1 hour. Next, the complex was analyzed by gel filtration using a Superdex 200 10/300 GL analytical column (GE Healthcare). Proteins were eluted in fractions of $500 \mu \mathrm{l}$ in a final volume of $18 \mathrm{ml}$.

From each fraction, $200 \mu \mathrm{l}$ were transferred to a 96 -well plate to check for the presence of fNup214NES fluorescent signal using a CLARIOstar Plus plate reader (BMG labtech). The remaining volume was precipitated at $-20^{\circ} \mathrm{C}$ for 2 hours using 3 volumes of ice-cold acetone, followed by centrifugation at $13000 \mathrm{~g}$ for 10 minutes. The acetone was then discarded and the pellet was left to air dry. The pellet was re-suspended in $40 \mu 1$ of 4x SDS sample buffer (125 
$\mathrm{mM}$ Tris $\mathrm{pH} 6.8,4 \% \mathrm{SDS}, 0.02 \%$ Bromophenol blue, $10 \%$ glycerol) and heated at $70^{\circ} \mathrm{C}$ for 2 minutes. $15 \mu 1$ volumes were loaded on $4-20 \%$ bio-rad gradient gel (see section 1.6.2.6).

\subsubsection{Pulldown of purified Nup214 and CRM1 proteins}

Pulldown of MBP-Nup214 fragments was performed using amylose resin (\#E8021S, New England Biolabs) equilibrated in binding buffer (50 mM Tris $\mathrm{HCl}, \mathrm{pH} 7.4,200 \mathrm{mM} \mathrm{NaCl}, 1$ $\mathrm{mM} \mathrm{MgCl}_{2}, 5 \%$ glycerol supplemented with $1 \mathrm{mM} \mathrm{DTT}$ and $20 \mathrm{mg} / \mathrm{ml} \mathrm{BSA}$ ). For each reaction, 100 pmol of MBP-Nup214 was immobilized on the beads for 90 minutes, followed by washing 3 times in pulldown buffer ( $50 \mathrm{mM}$ Tris $\mathrm{HCl}, \mathrm{pH} 7.4,200 \mathrm{mM} \mathrm{NaCl}, 1 \mathrm{mM} \mathrm{MgCl} 2,5 \%$ glycerol supplemented with $1 \mathrm{mM}$ DTT) and putative binding partners were added to the immobilized MBP-Nup214 in a final volume of $500 \mu 1$ of pulldown buffer. The bead-protein mixture was then incubated on a rotor for 6 hours at $4^{\circ} \mathrm{C}$. Following the incubation time, the beads were washed 3 times in pulldown buffer. All washing steps were done by centrifugation at $300 \mathrm{~g}$ for 5 minutes. Bound proteins were eluted with $25 \mu$ of SDS sample buffer and then loaded with a $5 \%$ input on a $4-20 \%$ gradient gel as previously described. The resulting gel was stained with Coomassie (see section 1.6.2.6).

Pulldown of CRM1-GST was performed using Glutathione Sepharose 4B resin (GE Healthcare) following the same washing and incubation steps and using the same buffers described in the previous paragraph.

All proteins used for pulldown reactions were added at an equimolar ratio of 1:1 with a final concentration of 100 pmol for each protein.

\subsubsection{Coomassie staining of SDS gels}

Gels were fixed for 10 minutes in a fixing solution (10\% ethanol, $5 \%$ acetic acid in water) followed by staining using $400 \mu \mathrm{l}$ of Coomassie buffer (0.5\% G250 Coomassie brilliant blue and $0.25 \%$ R250 Commassie brilliant blue in $100 \% \mathrm{EtOH}$ ) supplemented in a total volume of $50 \mathrm{ml}$ fixer buffer. The gel was boiled in the Coomassie buffer using a microwave and shaken for 10 minutes. The Coomassie buffer was discarded and replaced by water. The stained gel was scanned the next day. 


\subsubsection{Cell biology based assays}

\subsubsection{Cell lines}

HeLa P4 (Charneau et al., 1994) and COS7 (Gluzman, 1981) cells were used for immunofluorescence, HeLa cells were used for proximity ligation assays (PLA) and Hek293T cells (DuBridge et al., 1987) were used for immunoprecipitation. All cells were maintained in Dulbecco's Modified Eagle Medium (DMEM, ThermoFisher Scientific) supplemented with 5\% FCS, $100 \mathrm{I} . \mathrm{U} . / \mathrm{ml}$ penicillin and $100 \mu \mathrm{g} / \mathrm{ml}$ streptomycin and incubated at $37^{\circ} \mathrm{C}$ and $5 \% \mathrm{CO}_{2}$. Hek293-Flp-In cells (Weterman et al., 2001) used for stable expression of cells, were maintained in Dulbecco's Modified Eagle Medium (DMEM) supplemented with 5\% FCS, 100 I.U./ml penicillin and $100 \mu \mathrm{g} / \mathrm{ml}$ streptomycin and $10 \mathrm{ng} / \mathrm{ml}$ Blastocidin $\mathrm{S} \mathrm{HCl}$ (ThermoFisher Scientific)

\subsubsection{Transfection of cell lines}

For immunofluorescence experiments, either Calcium chloride $\left(\mathrm{CaCl}_{2}\right)$ or lipofectamine transfection was used, while for immunoprecipitation, transfection was done following the $\mathrm{CaCl}_{2}$ transfection protocol.

\section{- Calcium chloride $\left(\mathrm{CaCl}_{2}\right)$ transfection:}

$\mathrm{CaCl}_{2}$ transfection was done following a standard protocol (Chen, 2011). The amounts of DNA and reagents used, were adjusted according to the experiment conditions.

For immunofluorescence experiments, $1 \mu \mathrm{g}$ of DNA was supplemented with $20 \mu 1$ of $250 \mathrm{mM}$ $\mathrm{CaCl}_{2}$ and then an equal volume of $2 \mathrm{x} \mathrm{HBS}\left(50 \mathrm{mM}\right.$ Hepes, $250 \mathrm{mM} \mathrm{NaCl}, 1.5 \mathrm{mM} \mathrm{Na}_{2} \mathrm{HPO}_{4}$, $\mathrm{pH}$ 6.94) was added to the $\mathrm{CaCl}_{2}$-DNA mixture. The mixture was incubated for 25 minutes at room temperature and then added drop wise to the cells. Cells were incubated overnight at $37^{\circ} \mathrm{C}$ and $5 \% \mathrm{CO}_{2}$. Next day, the cells were prepared for immunofluorescent analysis (see section

\subsubsection{5).}

For immunoprecipitation experiments, the transfection was done following the same procedure as described before. In brief, $30 \mu \mathrm{g}$ of DNA was supplemented with $1125 \mu \mathrm{l}$ of $250 \mathrm{mM} \mathrm{CaCl}_{2}$ and then an equal volume of $2 \mathrm{x}$ HBS was added to the $\mathrm{CaCl}_{2}$-DNA mixture. The mixture was then incubated for 25 minutes at room temperature and added drop wise to the cells. Cells were incubated overnight at $37^{\circ} \mathrm{C}$ and $5 \% \mathrm{CO}_{2}$ and collected for immunoprecipitation (see section 1.6.3.10; 1.6.3.11). 


\section{- Lipofectamine transfection:}

Lipofectamine 2000 Transfection Reagent (ThermoFisher Scientific) was used according to the manufacturer's instructions. DMEM supplemented with 5\% FCS was used for plating the cells. The transfection reaction was prepared for a 24-well plate scale. The reaction was done by mixing $1 \mu 1$ of Lipofectamine with $50 \mu \mathrm{l}$ of Opti-MEM in one tube, while in another tube $1 \mu \mathrm{g}$ of the plasmid DNA of interest was mixed with $50 \mu \mathrm{l}$ of Opti-MEM. The components of the two tubes were mixed and incubated at room temperature for 20 minutes. Following the incubation period, the transfection mix was added to the wells drop wise. Due to the toxic nature of lipofectamine, the medium was replaced by fresh DMEM supplemented with 5\% FCS four hours following the transfection.

\subsubsection{Cell lines stably expressing GFP-Nup214 (L878A/L882A)}

Inducible expression of proteins was done by stable transfection of Hek293-Flp-In cell lines. The expression was controlled by a tetracycline promoter and the transfected plasmids have resistance against hygromycin B (ThermoFisher Scientific). To generate stable cell lines, Hek293-Flp-In cells were plated to a confluency of $40 \%$ in 6 -well plates. The cells were washed with pre-warmed 1x PBS and the media was replaced with $2 \mathrm{ml}$ of DMEM supplemented with glucose and fetal bovine serum 1 hour before the transfection. A transfection mixture was prepared by supplementing $9 \mu \mathrm{l}$ FuGENE® HD Transfection Reagent (Promega) in $91 \mu \mathrm{l}$ of Opti-MEM followed by incubation for $5 \mathrm{~min}$. $0.6 \mu \mathrm{g}$ of GFP-Nup214 (L878A/L882A) and 1.8 $\mu \mathrm{g}$ of pOG44 were added to the transfection mixture and the Fugene-DNA mixture was incubated for 15 minutes, then added drop wise to the 6-well plates. The cells were incubated at $37^{\circ} \mathrm{C}$ and $5 \% \mathrm{CO}_{2}$ to confluency. Once confluent, the cells were expanded and selection with $100 \mu \mathrm{g} / \mathrm{ml}$ of hygromycin B was started immediately. To ensure the integration of the DNA into the cell genome, $10 \mu \mathrm{g} / \mathrm{ml}$ of Blastocidin $\mathrm{S} \mathrm{HCl}$ was added to the cells. DMEM supplemented with the selection antibiotic was continuously exchanged to remove dying cells and maintain the selection process. The plates were monitored for the formation of colonies under the microscope. Once formed, single colonies were scratched by pipet tips and transferred to 6-well plates, where they were maintained until confluency.

The cells were induced using tetracycline $\mathrm{HCl}$ (Sigma-Aldrich) at a concentration of $0.1 \mathrm{ug} / \mathrm{ml}$ and then incubated for 24 hours at $37^{\circ} \mathrm{C}$ and $5 \% \mathrm{CO}_{2}$. Expression levels of GFP-Nup214 (L878A/L882A) were tested by Western blotting (see section 1.6.3.6). 


\subsubsection{RNAi}

RNAi targeting Nup214 and Nup358 were ordered and a reverse transfection was done using Lipofectamine RNAiMAX Transfection reagent (ThermoFisher Scientific). The transfection reaction was prepared by mixing $1 \mu 1$ of Lipofectamine RNAiMAX with $9 \mu 1$ of Opti-MEM in one tube, while in another tube $1.75 \mu \mathrm{l}$ of $20 \mathrm{uM}$ of the siRNA of interest was mixed with 38.25 $\mu 1$ of Opti-MEM. The components of the two tubes were mixed and incubated at room temperature for 20 minutes. Following the incubation period, the transfection mix was added to the wells and a total of 100.000 cells were plated on top. A second round of siRNA transfection was performed 48 hours after the initial transfection, following the same procedure. 24 hours later, cells were analyzed by immunofluorescence (see section 1.6.3.5).

All transfections were done in an antibiotic free DMEM. Western blotting was used to determine the efficiency of the knockdowns.

In case of overexpression experiments, $1 \mu \mathrm{g}$ of plasmid DNA was included during the second round of transfection, together with the siRNA.

\subsubsection{Immunofluorescence}

Cells were seeded on coverslips at a density of 70.000 cells per one well of a 24 -well plate. The coverslips were washed using $1 \mathrm{X}$ PBS and then fixed for 20 minutes at room temperature using $3.7 \%$ formaldehyde. The cells were then washed again three times using $1 \mathrm{X}$ PBS followed by permeabilization for 5 minutes using $0.5 \%$ triton X-100 in 1X PBS. To block unspecific sites, coverslips were incubated with $3 \%$ bovine serum albumin (BSA) in $1 \mathrm{X}$ PBS at room temperature for 1 hour. Subsequently, the coverslips were incubated with the primary antibodies (see Table 12) at room temperature for 1 hour. The coverslips were then washed three times using 1\% BSA. Coverslips were incubated with fluorescently tagged secondary antibodies (see Table 13) for 1 hour at room temperature. Next, they were washed three times with $1 \mathrm{X}$ PBS and three times with autoclaved water. The coverslips were mounted using Mowiol (Sigma-Aldrich) mounting medium supplemented with DAPI (Merk) and antibody staining was visualized using an LSM 510 meta confocal microscope (Zeiss).

\subsubsection{Western blotting}

Cell lysates were prepared using NP40 buffer (50 mM Tris $\mathrm{HCl}, \mathrm{pH} 8.8,150 \mathrm{mM} \mathrm{NaCl}, 1 \%$ NP40 supplemented with $1 \mathrm{mg} / \mathrm{ml} \mathrm{AP,} 1 \mathrm{mg} / \mathrm{ml} \mathrm{LP}, 1 \mathrm{mM}$ DTT and $1 \mathrm{mM} \mathrm{PMSF}$ ) and allowed to lyse on ice for 30 minutes. After the incubation, the cell lysates were centrifuged at 16.000 
$\mathrm{g}$ for 30 minutes. The pellet was discarded and the supernatant was collected for Western blot analysis.

Whenever equal amounts of proteins were required, the protein concentration was first measured using Pierce BCA protein assay kit (ThermoFisher Scientific) and equal amounts of proteins were loaded on a $4-20 \%$ bio-rad gradient gel. Following the separation, the proteins were transferred to an Amersham Protran 0.45 NC nitrocellulose membrane (GE Healthcare). For proteins with molecular weights below 150 kilodaltons, the transfer was performed at 100 volts for 90 minutes while proteins with higher molecular weights were transferred at 20 volts for 16 hours. Following the transfer, the membrane was blocked for 1 hour in $5 \%$ milk in $1 \mathrm{X}$ TBS-T (see section 1.6.3.6) and then probed using specific primary antibodies (see Table 12) by an overnight incubation at $4^{\circ} \mathrm{C}$. Next, the membrane was washed three times in $1 \mathrm{X}$ TBS-T (50 mM TrisHCl, $\mathrm{pH} 7.6,150 \mathrm{mM} \mathrm{NaCl}, 0.1 \%$ tween-20) and subsequently probed with secondary antibodies conjugated to a fluorophore (see Table 13: Secondary antibodies). The membrane was finally washed again in $1 \mathrm{X}$ TBS-T and then visualized using the Odyssey ${ }^{\circledR} \mathrm{Sa}$ Infrared Imaging System (LI-COR).

\subsubsection{Proximity ligation assay (Gullberg et al., 2004)}

A total number of $70.000 \mathrm{HeLa}$ cells per well of a 24 -well plate were seeded on poly-L-lysine coated coverslips. The Duolink proximity ligation assay (PLA, Sigma-Aldrich) kit was used to determine the proximity of two proteins of choice. All procedures were performed according to the manufacturer's guidelines.

The cells were fixed using $3.7 \%$ formaldehyde, permeabilized using $0.5 \%$ triton $\mathrm{X}-100$ in $1 \mathrm{X}$ PBS and then blocked for 30 minutes using the Duolink blocking solution. The cells were subsequently incubated with two primary antibodies of interest for one hour at room temperature. All antibody dilutions (see Table 12) were prepared in Duolink II antibody diluent. The primary antibody was removed by performing a two times wash using $120 \mu \mathrm{l}$ of wash buffer A (10 mM Tris $\mathrm{HCl}, \mathrm{pH} 8.0,150 \mathrm{mM} \mathrm{NaCl}, 0.05 \%$ Tween-20) for 5 minutes each. For each coverslip, a $40 \mu 1$ reaction of diluted PLA probes was prepared at a dilution of 1:5 and the coverslips were incubated at $37^{\circ} \mathrm{C}$ for 1 hour. Different combinations of Duolink PLA probes were used including a combination of secondary anti-mouse and anti-rabbit or secondary antigoat and anti-rabbit.

Ligation of the Duolink PLA probes was performed using Duolink ligase. A ligation mixture was prepared by diluting the ligation buffer at a ratio of 1:5 and the ligase at a ratio of 1:40 in autoclaved water. The coverslips were washed twice using $120 \mu \mathrm{l}$ of wash buffer A for 5 
minutes each and then $40 \mu \mathrm{l}$ volumes of the ligation mixture were added to each coverslip. All coverslips were incubated at $30^{\circ} \mathrm{C}$ for 30 minutes.

Next, the coverslips were washed again as described above and incubated with amplification polymerase, which contains the Duolink II In Situ detection reagent Red. The amplification mixture was prepared by diluting the amplification buffer at a ratio of $1: 5$ and the polymerase at a ratio of 1:80 in autoclaved water. To each coverslip, $40 \mu 1$ of the amplification mixture was added and the coverslips were incubated at $37^{\circ} \mathrm{C}$ for $1: 40$ hours. Finally, the coverslips were washed twice using $120 \mu \mathrm{l}$ of wash buffer B (200 mM Tris HCl, pH 7.5, $100 \mathrm{mM} \mathrm{NaCl}$ ) for 10 minutes each, followed by a single wash for 1 minute with $0.01 \mathrm{X}$ wash buffer B.

The coverslips were mounted using the DAPI containing Duolink II mounting medium. The proximity interactions were visualized with a confocal microscope (Zeiss) as fluorescent dots ( $\lambda$ excitation/emission $594 / 634 \mathrm{~nm}$ ). Data were analyzed using CellProfiler (see section 1.6.3.8).

\subsubsection{CellProfiler}

The quantification of proximity ligation assay (PLA) interactions was done using the CellProfiler software (Carpenter et al., 2006). A pipeline was generated to count the number of PLA interactions at the nuclear envelope (NE). DAPI channel was used to identify the cell nuclei with the "IdentifyPrimaryObject" module. The diameter of the objects was set to 80-150 pixels. Two-class Otsu adaptive thresholding was selected. Intensity values were used to distinguish the clumped objects. The smoothing filter for declumping and the minimum allowed distance between local maxima was calculated automatically. The cells were identified by the "IdentifySecondaryObjects" module using the Distance-N method where the nuclear area was expanded by 80 pixels. The cytoplasm area was defined using the "IdentifyTertiaryObjects" module and was obtained by subtracting the nuclear area from the cell area. The "IdentifyPrimaryObjects" module was used to define the PLA dots using PLA images. The diameter of the PLA dots was set to 2-10 pixels. Two-class Otsu adaptive thresholding was selected with a minimum threshold of 0.15 . Intensity values were used to distinguish the clumped objects as described for the cell nuclei. The "MaskImage" module was used to distinguish the PLA dots in the nucleus and cytoplasm. The "RelateObjects" module was used to relate the identified PLA dots in the cell, the nucleus and the cytoplasm to the identified cell, nuclear and cytoplasmic area, respectively. The "ExportToSpreadsheet" module was used to export the data and GraphPad Prism software was used to plot the data. Incomplete cells and cells touching the border of an image were excluded from the analysis. 


\subsubsection{Confocal microscopy}

A Zeiss LSM 510 meta confocal microscope based on the Axiovert 200M with a 63x PlanNeofluar 1.3 NA water-corrected objective with appropriate filter settings was used to acquire images. The objective was adjusted to the water-based Immersol W 2010 immersion oil. The $\mathrm{X}$-cite 120 mercury lamp series was used as an excitation light source. Filter settings were adjusted based on the fluorophores used in such a way to avoid cross-detection between the channels. A maximum of four different channels could be used for image acquisition. A Diodelaser with an excitation wavelength of $405 \mathrm{~nm}$ was used to detect DAPI. The Argon488 laser with an excitation wavelength of $488 \mathrm{~nm}$ was used to detect EGFP and secondary antibodies conjugated to AlexaFluor488 dye. The HeNe594 laser with an excitation wavelength of 594 $\mathrm{nm}$ was used to detect mCherry-tagged proteins, RFP-tagged proteins and secondary antibodies conjugated to AlexaFluor594. The HeNe633 laser with an excitation wavelength of $633 \mathrm{~nm}$ was used to detect secondary antibodies conjugated to AlexaFluor633 and AlexaFluor 647. The laser power was adjusted depending on the fluorescent signal intensity. The diameter of the pinhole was set at 1 airy units for all channels in use. The amplifier offset value was adjusted to control the background signal using the LSM software palette tool. The amplifier gain value and the transmission value were used to control the channel intensity. The data depth was set at 8 bit and the scan speed was set at $5 \mu \mathrm{s} /$ pixel. Four images per channel were averaged using the averaging mean function. Acquired images were analyzed with the ImageJ software.

\subsubsection{Immunoprecipitation of overexpressed Nup214}

Hek293T cells were seeded in $15-\mathrm{cm}$ plates until 50-60\% confluency. For each immunoprecipitation reaction, three $15-\mathrm{cm}$ plates were used. Cells were transfected with GFPor RFP-tagged Nup214 fragments using the calcium chloride transfection protocol (see section 1.6.3.2). The cells were collected in NP40 buffer ( $50 \mathrm{mM}$ Tris $\mathrm{HCl}, \mathrm{pH} 8.8,150 \mathrm{mM} \mathrm{NaCl}, 1 \%$ NP40 supplemented with $1 \mathrm{mg} / \mathrm{ml} \mathrm{AP,} 1 \mathrm{mg} / \mathrm{ml} \mathrm{LP,} 1 \mathrm{mM}$ DTT and $1 \mathrm{mM}$ PMSF), allowed to lyse on ice for 30 minutes and then centrifuged at $16.000 \mathrm{~g}$ for 30 minutes. The supernatant was collected and the pellet was discarded.

Immunoprecipitations were performed using magnetic GFP or RFP beads (Chromotek). For each reaction, $20 \mu \mathrm{l}$ of beads were equilibrated in $500 \mu \mathrm{l}$ of NP40 buffer. The collected supernatant was added to the equilibrated beads and the mixture was incubated at $4^{\circ} \mathrm{C}$ on a rotor for 90 minutes. Following the incubation time, the beads were washed three times in NP40 buffer and then supplemented in $25 \mu$ of SDS sample buffer. A 5\% input fraction and an unbound fraction were collected and run alongside with the bound fraction on a 4-20\% gradient 
gel. The proteins were then transferred to a nitrocellulose membrane as previously described (see section 1.6.3.6). In brief, the membrane was blocked using 5\% milk in $1 \mathrm{X}$ TBS-T and then incubated overnight at $4^{\circ} \mathrm{C}$ with anti-GFP (Chromotek, 1:1000), anti-RFP (Chromotek, 1:1000) and anti-Nup88 (BD Pharmingen, 1:1000) primary antibodies (see Table 12: Primary antibodies). Next, the membranes were incubated for one hour with fluorescently tagged secondary antibodies (see table Table 13: Secondary antibodies) against mouse IgG1 and rat IgG and finally visualized using the Odyssey® Sa Infrared Imaging System (LI-COR).

\subsubsection{Immunoprecipitation of stably expressed GFP-Nup214 (L878A/L882A)}

Hek293T-Flp-In cells stably expressing GFP-Nup214 (L878A/L882A) were seeded in 15-cm plates. For each immunoprecipitation reaction, three $15-\mathrm{cm}$ plates were used. Cells were induced using $1 \mathrm{ug} / \mathrm{ml}$ tetracycline and incubated to express the protein overnight at $37^{\circ} \mathrm{C}$ and $5 \% \mathrm{CO}_{2}$. The cells were collected in NP40 buffer, lysed on ice for 30 minutes and then centrifuged at $16.000 \mathrm{~g}$ for 30 minutes. The supernatant was collected and the pellet was discarded. Immunoprecipitations were performed using magnetic GFP beads (Chromotek) as above.

\subsubsection{Cell cycle analysis}

Cells were seeded on coverslips at a density of 70.000 cells per one well of a 24 -well plate. A double thymidine block was performed to arrest the cells in early S-phase. At 25-30\% confluency, HeLa cells were washed twice with 1x PBS and full DMEM supplemented with 2 $\mathrm{mM}$ thymidine was added for 18 hours. After the first thymidine block, the cells were released back into the cell cycle by washing with 1x PBS to remove the thymidine, followed by addition of fresh DMEM (10\% FCS, 1\% Pen-Strep) for 9h. During the 9 hours of release, the cells were transfected with the intended plasmids. A second block was performed for 17 hours by adding full DMEM supplemented with $2 \mathrm{mM}$ thymidine. Following the second thymidine block, the cells were released back into the cell cycle by washing with 1x PBS to remove the thymidine and adding fresh DMEM. One coverslip was fixed every two hours over a period of 24 hours. The cells were stained according to the previously described immunofluorescence protocol (see section 1.6.3.5).

\subsubsection{Export assay}

A total number of $80.000 \mathrm{HeLa} \mathrm{P} 4$ cells per well of a 24 well plates were seeded on coverslips. The cells were transfected with GFP-Nup214 (L878A/L882A)-Nup214 NES or GFP-Nup214 
(L878A/L882A)-Rev NES via a standard $\mathrm{CaCl}_{2}$ transfection protocol as previously described (see section 1.6.3.2). When needed, LMB was added at a final concentration of $10 \mathrm{nM}$ for 2 hours at $37^{\circ} \mathrm{C}$ and $5 \% \mathrm{CO}_{2}$.

Permeabilization of the cell membrane was performed using $0.0075 \%$ digitonin. Digitonin was prepared by diluting $7.5 \mu \mathrm{l}$ of $1 \%$ digitonin in $1 \mathrm{ml}$ of transport buffer $(20 \mathrm{mM}$ Hepes, $\mathrm{pH} 7.3$, $110 \mathrm{mM} \mathrm{KOAc}, 2 \mathrm{mM} \mathrm{Mg}(\mathrm{OAc})_{2}, 1 \mathrm{mM}$ EGTA). The coverslips were transferred to an icecold metal block. $60 \mu \mathrm{l}$ of diluted digitonin was added to each coverslip for 5 minutes. The permeabilization efficiency was monitored using trypan blue. Digitonin was removed by washing the coverslips three times using $1 \mathrm{x}$ transport buffer with at least one wash for 5 minutes. Next, the coverslips were transferred to a humidity chamber either at room temperature or at $4^{\circ} \mathrm{C}$, according to the experimental setup. The coverslips were incubated for 30 minutes with $1 \mu \mathrm{l}$ ATP regenerating system diluted in $39 \mu \mathrm{l}$ X transport buffer. The ATP regenerating system was composed of a mixture of $5 \mu \mathrm{l}$ of $2000 \mathrm{U} / \mathrm{ml}$ creatine-kinase (CPK) enzyme, $10 \mu \mathrm{l}$ of $80 \mathrm{mg} / \mathrm{ml}$ creatine-phosphate and $10 \mu 1$ of $100 \mathrm{mM} \mathrm{ATP} \mathrm{(disodium).} \mathrm{Later,} \mathrm{the} \mathrm{coverslips}$ were washed one time with $1 \mathrm{x}$ transport buffer and the protein mixture was added in a total volume of $40 \mu \mathrm{l}$ to each coverslip. The coverslips were then washed three times with $1 \mathrm{x}$ transport buffer and fixed with 3.7\% formaldehyde. Finally, the cells were either mounted directly on glass slides or proteins of interest were visualized by immunofluorescence (see section 1.6.3.5). 


\section{Results}

\subsection{A nuclear export sequence in Nup214 promotes its targeting to the} nuclear pore complex

\subsubsection{Expression of Nup214 results in cytoplasmic/ Nuclear envelope localization}

Nup214 is a protein of the cytoplasmic side of the NPC. The sequence of Nup214 can be divided into three parts: the ß-propeller domain comprising the first third of the protein, the coiled-coil domain comprising the center of the structure and the FxFG-repeat stretch comprising the Cterminus domain. Previous studies have determined the localization of endogenous Nup214 to be at the nuclear envelope, more specifically at the outer side of the nuclear envelope (Kraemer et al., 1998). By staining for Nup214, we could show that Nup214 localizes mainly at the nuclear envelope (Figure 5).

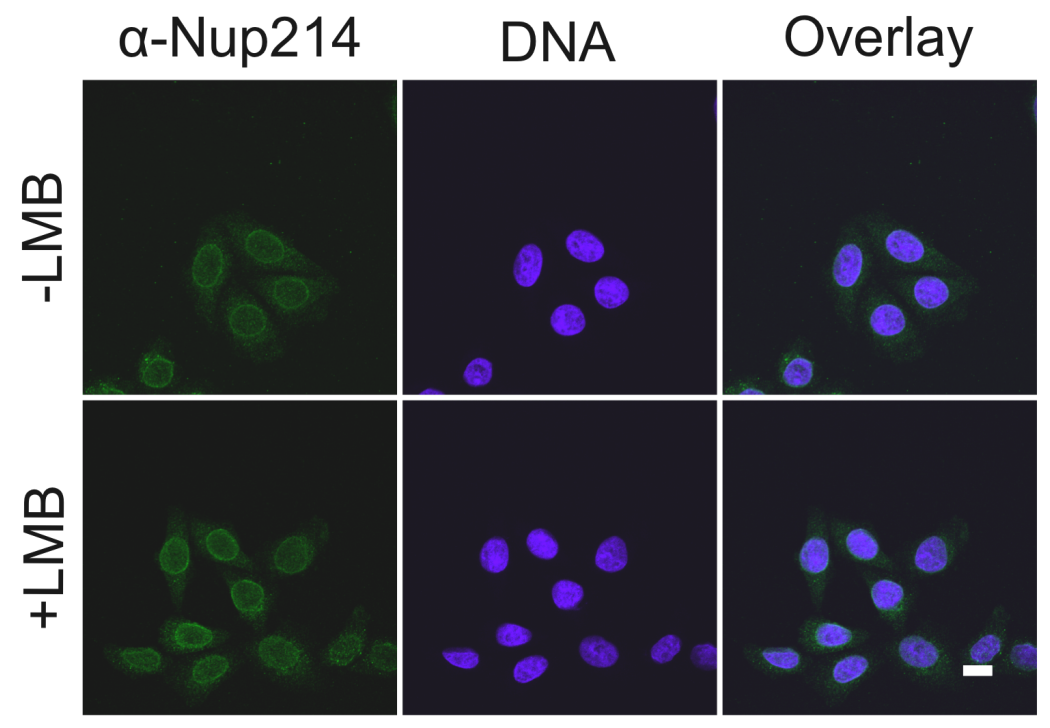

Figure 5: Nup214 is mainly localized at the NE. HeLa P4 cells were treated with or without $10 \mathrm{nM} \mathrm{LMB}$ for 2 hours, as indicated. The cells were fixed and immunostained with an anti-Nup214 antibody. The scale bar corresponds to $10 \mu \mathrm{m}$.

Previous studies described the localization of endogenous Nup214 to be in the cytoplasm (Shah et al., 1998, Hutten et al., 2006). Furthermore, the Nup214 sequence contain hydrophobic regions such as the FG-repeats which may interact with the central channel FG-repeats. To determine the localization pattern of Nup214, plasmids coding for full length and fragments of Nup214 (Figure 6a) were overexpressed in HeLa cells (Figure 6b). 
A

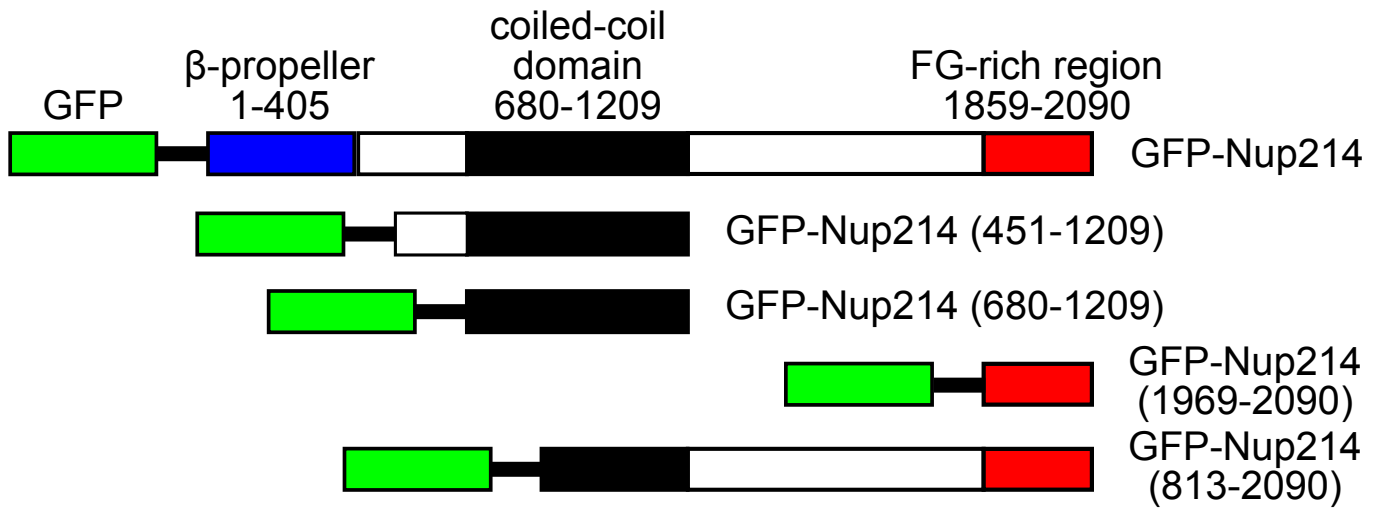

$\mathrm{B}$

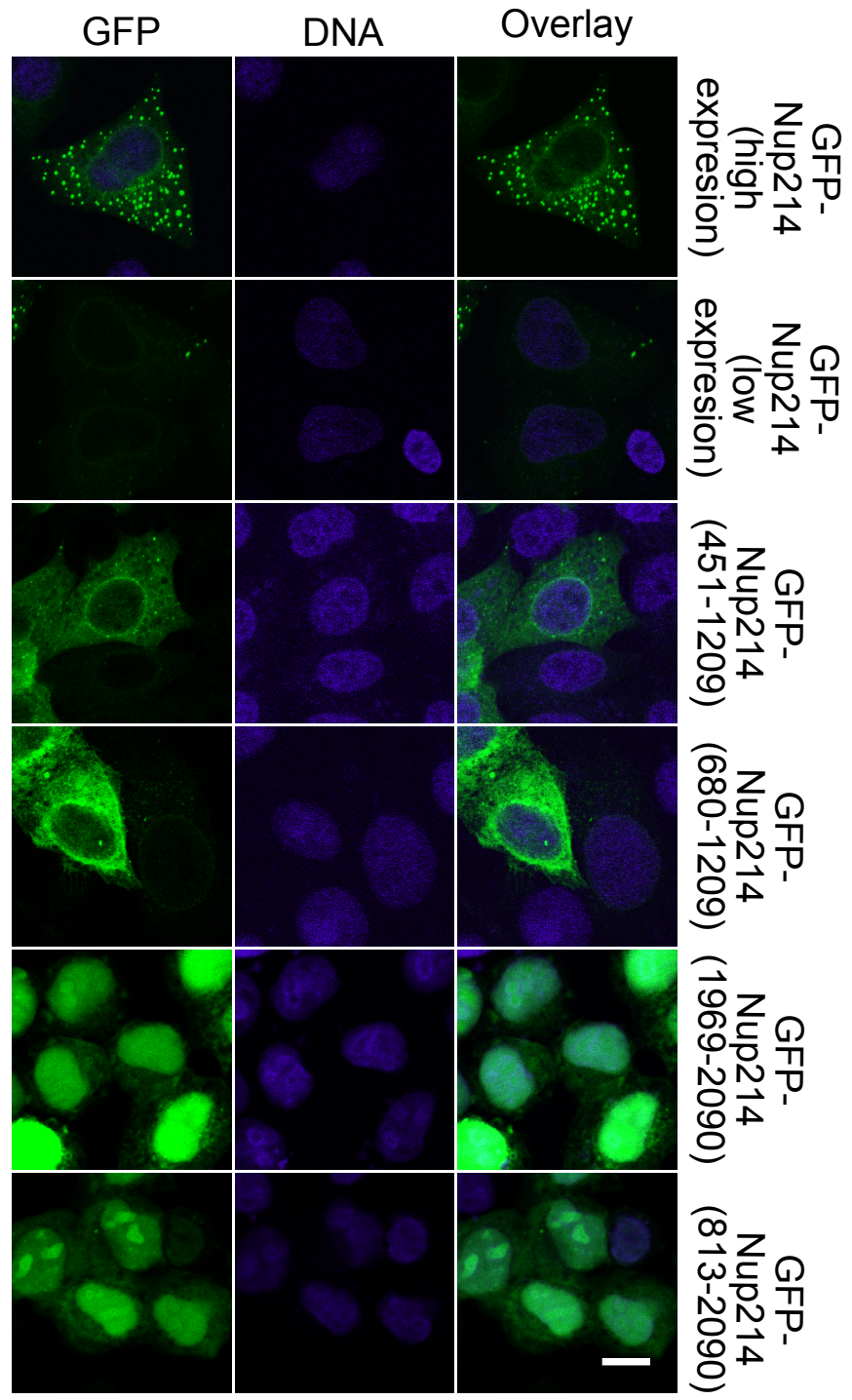

Figure 6: Different domains of Nup214 show different localizations. (A) We constructed GFP tagged full length Nup214 and different GFP tagged fragments of Nup214. The GFP-Nup214 (650-1209) represents the coiled-coil domain region, the GFP-Nup214 (481-1209) represent the coiled-coil domain and a small part of the N-terminus end, the GFP-Nup214 (19692090) represents most of the FG-repeat domain of Nup214 and GFP-Nup214 (890-2090) which represents the FG-repeat domain and part of the coiled-coil domain. (B) HeLa P4 cell were transfected with different GFP constructs as indicated and analyzed by confocal microscopy. The scale bar corresponds to $10 \mu \mathrm{m}$. 
When Nup214 was overexpressed, it tends to localize to the cytoplasm and the nuclear envelope, depending on its level of expression. At low expression levels, Nup214 tends to show a localization at the nuclear envelope (Figure 6b; panel 2). At high expression levels, however, excessive Nup214 that had no free sites for insertion at the nuclear envelope tends to aggregate and form puncta in the cytoplasm. Overexpression of wild type full length Nup214 resulted in the formation of such aggregates. Overexpression of plasmids coding for Nup214 lacking the C-terminus FG-repeat region, resulted in the loss of these puncta (Figure 6b, panel 3-4).

Different fragments of Nup214 representing different regions of the protein were overexpressed. Next, GFP-Nup214 (1969-2090) was exclusively localized in the nucleus. Overexpression of GFP-Nup214 (813-2033) resulted in the distribution of Nup214 across the two cell compartments (figure $2 b$ ). On the other hand, overexpression of the plasmids coding for the coiled-coil domain of Nup214 resulted in an exclusive localization in the cytoplasm. GFP-Nup214 (680-1209) showed a cytoplasmic localization only while GFP-Nup214 (4511209) was additionally localized at the nuclear envelope. The additional amino acid sequence present before amino acid 680 in GFP-Nup214 (451-1209) may contribute to the insertion of the protein at the outer nuclear envelope.

In conclusion, our results confirmed the findings from previous studies. GFP-Nup214, GFPNup214 (451-1209) and GFP-Nup214 (680-1209) localized to the cytoplasm. In addition, only GFP-Nup214, GFP-Nup214 (451-1209) localized at the nuclear envelope. GFP-Nup214 (8132033) localized to the cytoplasm and the nucleus, while GFP-Nup214 (1969-2090) localized to the nucleus only.

\subsubsection{Nup214 has a nuclear export signal in its coiled coil region}

Our previous conclusion that Nup214 has the ability to access the nucleus through its FG-repeat region raised the question of what keeps Nup214 in the cytoplasm. As previously shown in many studies, several proteins possess a nuclear export signal (NES), which maintain their localization in the cytoplasm. Even though it is not a very common feature of nucleoporins, previous studies have shown that some nucleoporins such as Nup153 have a nuclear localization signal (NLS) that promotes their import to the nuclear side of the NPC (Nakielny et al., 1999). We hypothesized that Nup214 may contain an NES, which could maintain it out of the nucleus when overexpressed. To investigate this, cells were transfected with plasmids coding for full length Nup214 and then incubated with $10 \mathrm{nM}$ of leptomycin B (LMB) for 2 hours. LMB is an irreversible inhibitor of CRM1. It binds covalently to the cysteine 528 residue and inhibits the interactions of CRM1 with NES containing proteins, thus inhibiting their nuclear export (Kudo 
et al., 1998). Nup214 was suspected to be a CRM1 dependent substrate. Overexpression results showed that addition of LMB resulted in a dramatic increase in the nuclear localization of Nup214. Different versions of Nup214 with GFP-, mCherry- and myc-tag were expressed and the result was reproducible regardless of the tag (Figure 7). The addition of LMB resulted in the loss of the Nup214 puncta when using a GFP tag, while a similar effect was not observed when using a myc tag. Furthermore, addition of LMB did not affect endogenous Nup214 localization (Figure 5).

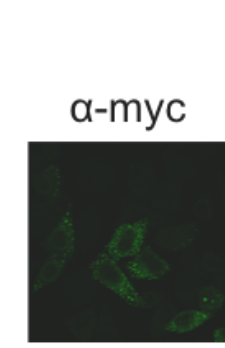

-LMB
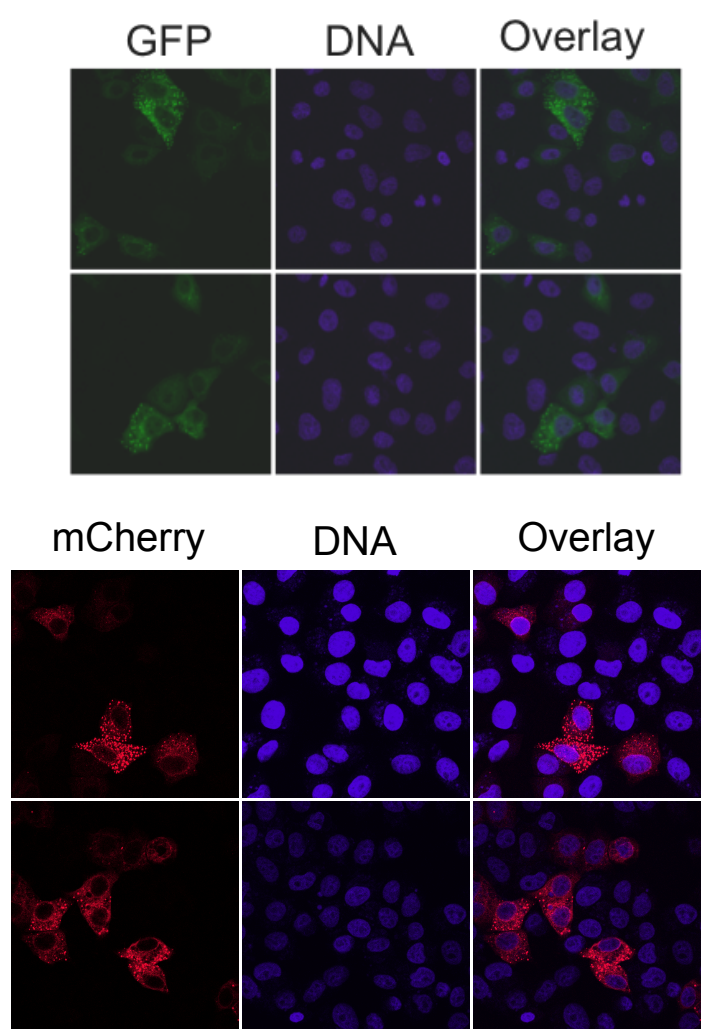

$+\mathrm{LMB}$
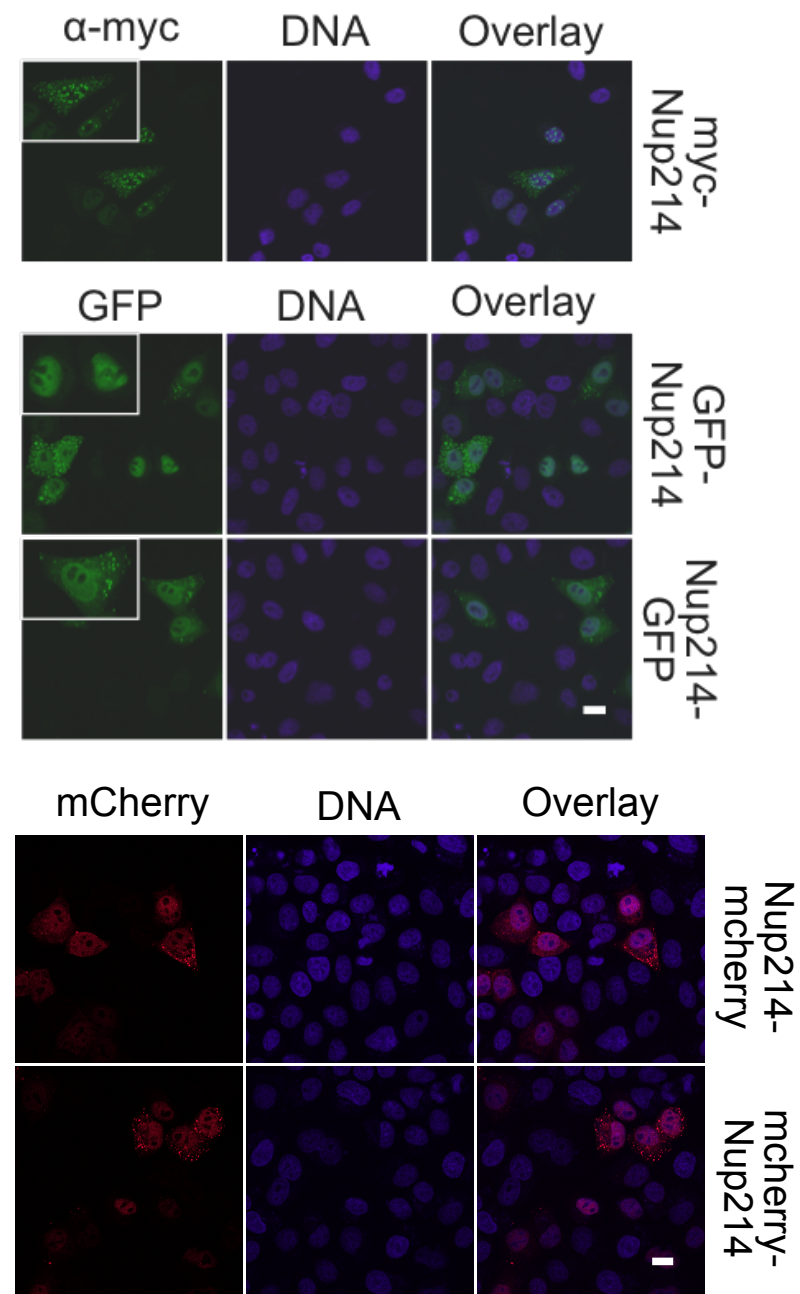

Figure 7: Inhibition of CRM1 export by LMB causes Nup214 to localize in the nucleus. HeLa P4 cells were transfected with the plasmids coding for myc-Nup214, GFP-Nup214, Nup214-GFP, mCherry-Nup214 and Nup214-mcherry. The cells were treated with or without LMB as indicated. Cells transfected with a myc-tagged plasmid were analyzed by indirect immunofluorescence using an anti-myc antibody. All cells were viewed using a confocal microscope. DNA was stained using DAPI. The different constructs used are indicated on the right side of each panel and the antibodies used are indicated on top of the panels. The scale bars correspond to $10 \mu \mathrm{m}$. 
Additionally, plasmid coding for full length of Nup214 tagged with mCherry at either the Nterminus or the C-terminus were used. Similar to the GFP tagged Nup214, we observed the same effect of LMB, shifting the mCherry tagged Nup214 to the nucleus (Figure 7).

In conclusion, myc-Nup214, GFP-Nup214, Nup214-GFP, mCherry-Nup214 and Nup214mcherry localized to the cytoplasm and the nuclear envelope. LMB treatment resulted in a clear nuclear localization of the fusion proteins.

\subsubsection{The Nup214 NES sequence is conserved across different species}

The Nup214 sequence was screened for an NES. Indeed, we found that Nup214 contains an NES region which follows the same hydrophobic spacing of a typical NES sequence. In addition, the NES sequence was highly conserved across vertebrates. The NES sequence is located in the coiled-coil region of Nup214 and comprises amino acids 875 to 889 of Nup214 sequence (Figure 8).

Next, we inhibited the functionality of the putative NES by mutating two leucine residues in this sequence, namely L878 and L882 to alanine.

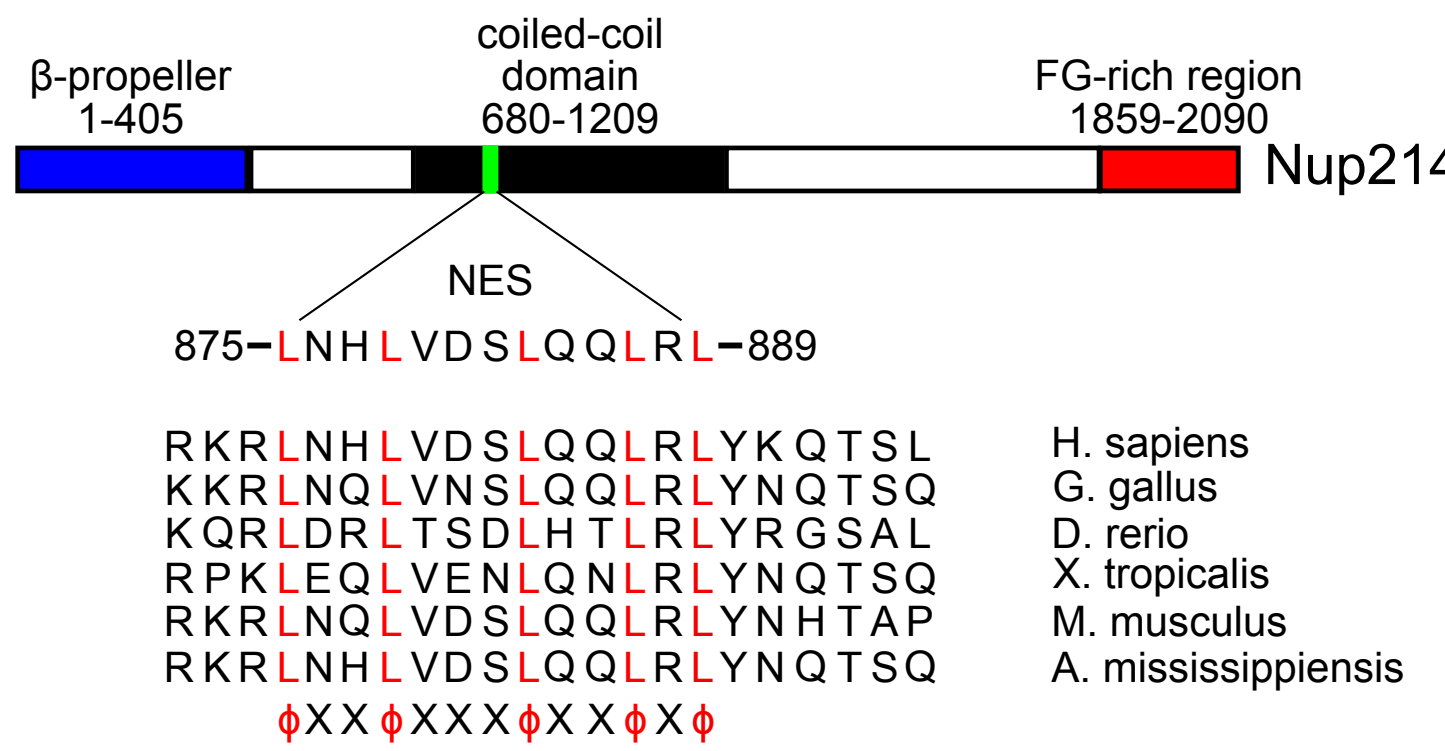

Figure 8: Nup214 NES is highly conserved across vertebrates. The Nup214 protein sequence of human was scanned for possible NES. The search revealed a conserved region between amino acids 875 and 889 , which follow the hydrophobic spacing of a classic NES. The identified sequence was compared in various vertebrates, as indicated. The hydrophobic residues $(\Phi)$ are represented in red and the hydrophobic spacing pattern is shown as ФХХФХХХФХХФХФ. The scan was performed by Sarah Port.

Plasmids coding for myc-Nup214 (L878A/L882A) and GFP-Nup214 (L878A/L882A) were overexpressed in HeLa cells to characterize their localization. The mutation of the leucine residues resulted in variable effects across different tags. Using myc-Nup214 (L878A/L882A), 
around $60 \%$ of the transfected cells have the protein localized inside the nucleus. With a GFPtag, the protein failed to show a similar result, may be due to the GFP-tag causing changes in the conformation of the protein or an increased solubility of the protein (Figure 9).
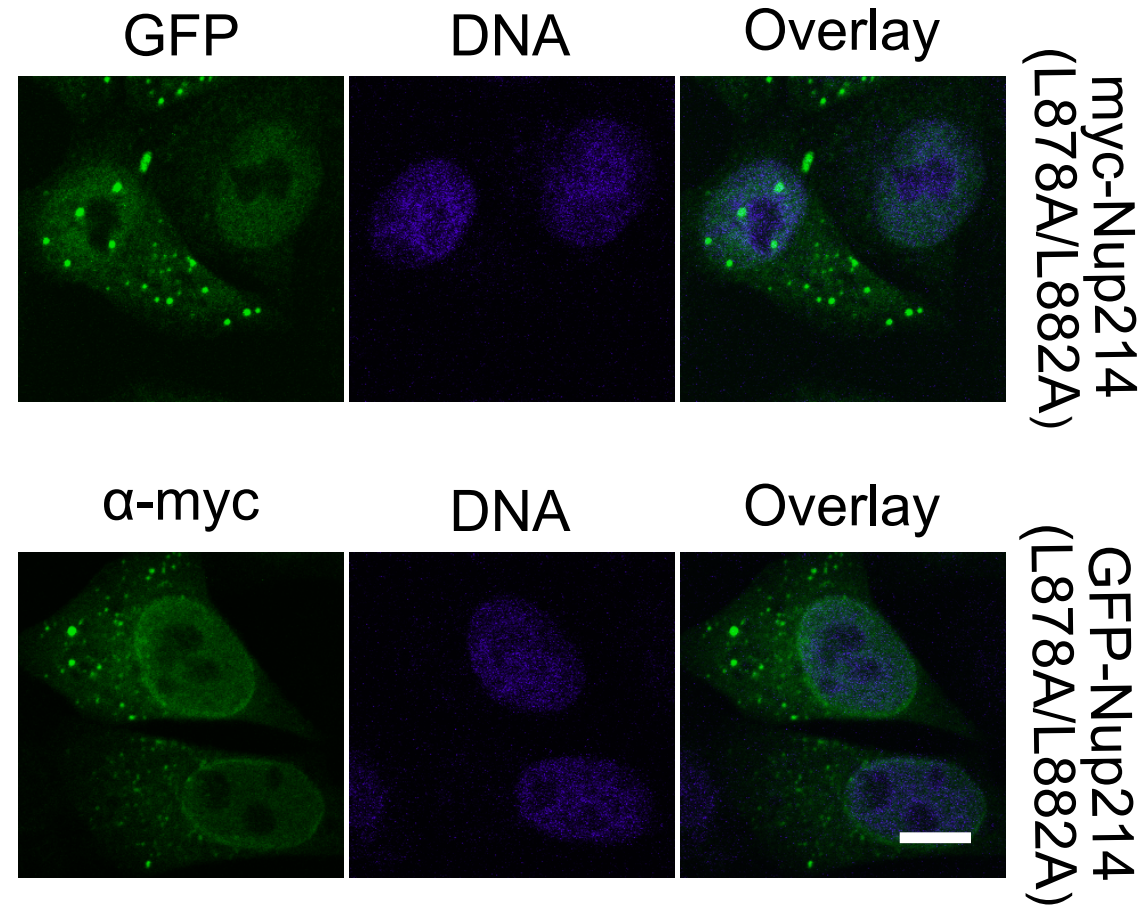

Figure 9: A mutation in Nup214 NES sequence leads to inhibition of Nup214 export. A mutation was introduced in the NES sequence of myc-Nup214 and GFP-Nup214. Two leucine residues L878 and L882 were exchanged for alanine. mycNup214 (L878A/L882A) and GFP-Nup214 (L878A/L882A) were transfected in HeLa P4 cells. Cells transfected with a myctagged plasmid were analyzed by indirect immunofluorescence using an anti-myc antibody. DNA was stained using DAPI. The scale bar corresponds to $10 \mu \mathrm{m}$.

\subsubsection{LMB resistant CRM1 localizes GFP-Nup214 back to the cytoplasm in LMB treated cells}

Following a different approach, we decided to overexpress plasmids coding for full length GFPNup214 together with the LMB resistant version of CRM1, CRM1_C528S-HA, in the presence of LMB. As we showed earlier, a full length GFP-Nup214 shifted its localization to the nuclear compartment in the presence of LMB. As shown in Figure 10, we detected a cytoplasmic localization of GFP-Nup214 enhanced by the LMB resistant CRM1 under conditions where LMB normally traps GFP-Nup214 in the nucleus. By overexpressing the LMB resistant CRM1, we ensured that CRM1 interaction to the NES region of the Nup214 is in fact specific and a reason for maintaining Nup214 in the cytoplasmic compartment. 


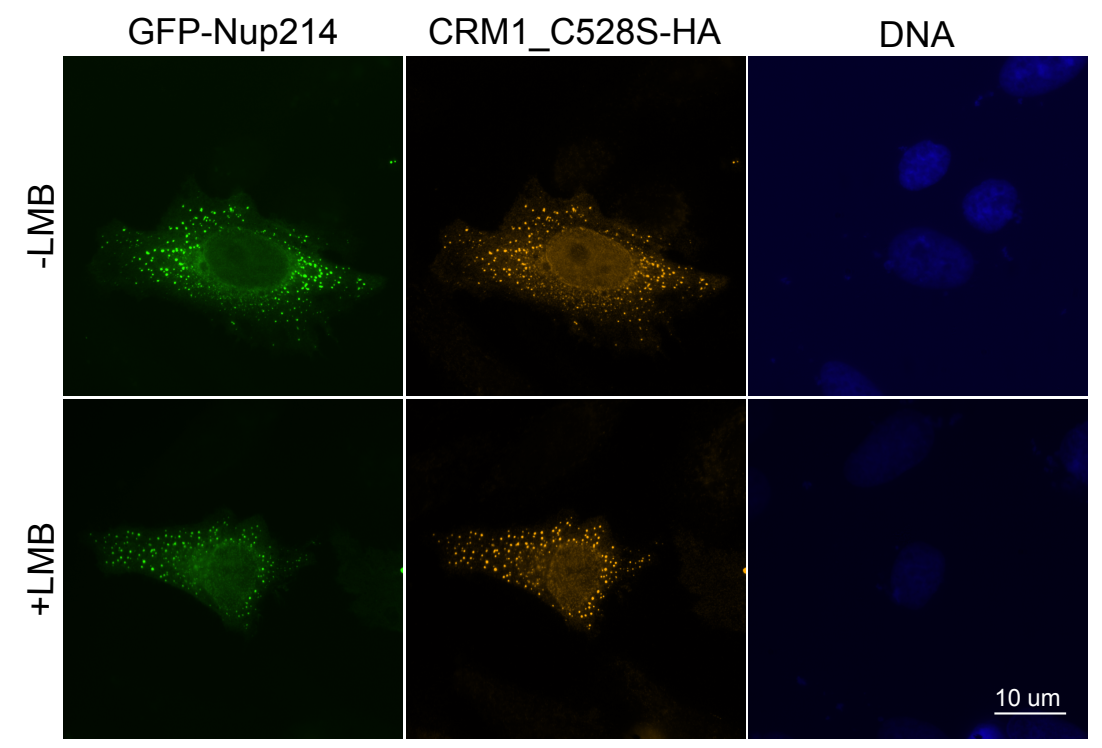

Figure 10: An LMB resistant CRM1 can rescue Nup214 in transfected cells. GFP-Nup214 and CRM1_C528S-HA were transfected in HeLa P4 cells. The cells were treated with or without $10 \mathrm{nM}$ LMB for 2 hours, as indicated. The cells were immunostained for the myc-tag. All cells were viewed using a confocal microscope. DNA was stained using DAPI. The scale bar corresponds to $10 \mu \mathrm{m}$.

\subsubsection{Regions other than the FG-repeat can give Nup214 access to the nucleus}

We wondered if other regions of Nup214 can affect the localization of Nup214 in the same manner as the FG-repeat domain (Figure 6). Plasmids coding for GFP-Nup214 (680-1209) and GFP-Nup214 (680-1209)_L878A_L882A were transfected in cells. Nuclear export was blocked with LMB. GFP-Nup214 (680-1209) showed an exclusive cytoplasmic localization (Figure 6 and Figure 11). Strikingly, GFP-Nup214 (680-1209) localized to the nucleus upon addition of LMB (Figure 11). Additionally, a mutation in the NES sequence of GFP-Nup214 (680-1209) by exchanging leucine 878 and 882 for alanine, yielded results similar to LMB addition. These results showed that Nup214 seems to have several regions in its sequence that can localize it to the nucleus. Yet the presence of an NES sequence, as shown in case of GFPNup214 (680-1209) fragment, was sufficient to maintain Nup214 in the cytoplasmic compartment of the cell. 


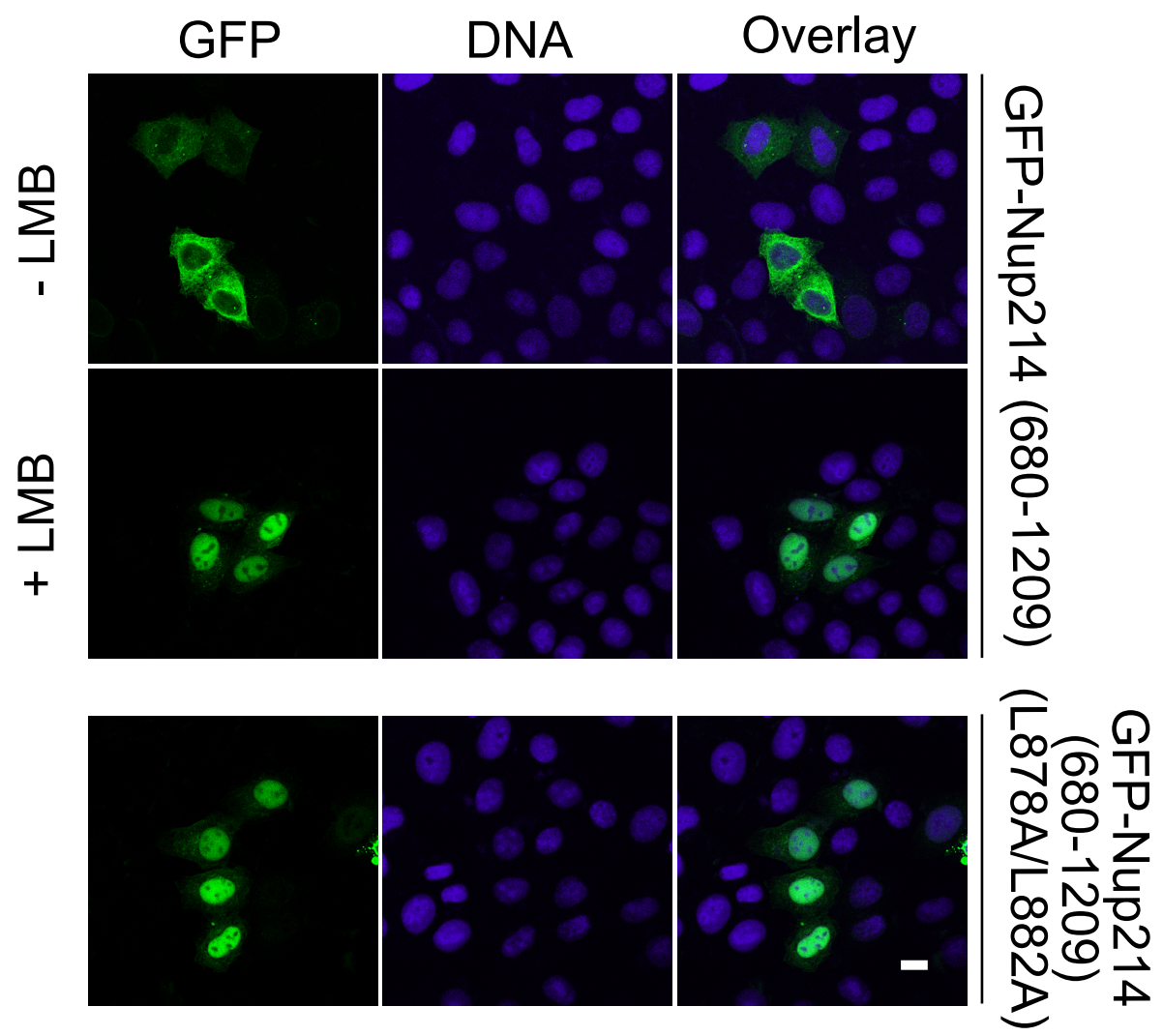

Figure 11: An isolated Nup214 coiled-coil fragment can localize to the nucleus under LMB. A plasmid coding for the coiled-coil domain fragment, GFP-Nup214 (680-1209), was transfected in HeLa P4 cells, which were then incubated with and without LMB, as indicated. Additionally, a mutation was introduced in the NES sequence of GFP-Nup214 (680-1209) by exchanging the two leucine residues L878 and L882 for alanine and the generated plasmid, GFP-nup214 (6801209)_L878A/L882A, was transfected in HeLa P4 cells. The scale bar corresponds to $20 \mu \mathrm{m}$.

\subsubsection{Nup214 interacts with CRM1 in an NES dependent manner}

Nuclear export signals (NES) are characteristic of CRM1 export cargos. The interactions of CRM1 with proteins containing an NES in their sequence such as protein kinase inhibitor protein (PKI, Wen et al, 1995), HIV-1 Rev NES (Fischer et al; 1995; Askjaer et al., 1998) and Snurportin-1 (SPN1) was characterized (Dong et al, 2009; Monecke et al., 2009). Our results suggested that Nup214 is a cargo of CRM1. We next investigated a possible direct interaction of Nup214 and CRM1 in an NES dependent manner. Different fragments of Nup214 representing the full coiled-coil domain or parts of it were purified. First, GST tagged Nup214 (680-1209), Nup214 (650-950) and Nup214 (585-1209) were purified. The GST tagged Nup214 fragments were hard to purify due to very low solubility of the protein. The purification steps were optimized by exchanging the GST-tag for an MBP-tag. The use of an MBP tag improved the purification scale significantly. The solubility of the protein was increased by addition of $0.1 \%$ of triton-X100.

The interaction of Nup214 and CRM1 via the FG-repeat region was previously studied and characterized (Fornerod et al., 1997, Xylourgidis et al., 2006). It was also shown that the 
addition of a Nup214 FG-repeat region helped to stabilize the formation of CRM1 dependent export complexes (Roloff et al., 2013; Port et al., 2015). To investigate if Nup214 interacts with CRM1 as an export complex in an NES dependent manner, we immobilized purified NEScontaining Nup214 fragments tagged with maltose binding protein (MBP). MBP-Nup214 (650950)-His was immobilized on MBP beads and incubated with CRM1-His or CRM1-His and Nup214 (1916-2033) or CRM1-His and Ran ${ }_{Q 69 L(1-180)}$ GTP or CRM1-His, Nup214 (1916-2033) and $\operatorname{Ran}_{\mathrm{Q} 69 \mathrm{~L}(1-180)} \mathrm{GTP}$ at an equimolar ratio. A complex of the purified MBP-tagged Nup214 fragment was successfully formed with CRM1, Nup214 (1916-2033) and $\operatorname{Ran}_{\mathrm{Q} 69 \mathrm{~L}(1-180)} \mathrm{GTP}$ (Figure 12a, lane 4). Fitting with previous findings, the presence of Nup214 (1916-2033) resulted in the formation of more amount of the complex (Figure 12a, lane $3 \& 4$ ) which point to the importance of the FG-repeat region for stabilization of a CRM1 dependent export complex. In the absence of $\operatorname{Ran}_{\mathrm{Q} 69 \mathrm{~L}(1-180) \mathrm{GTP}}$, the export complex failed to form, confirming that this complex indeed behaves as an export complex (Figure 12a, lane 2). As a control, purified MBP protein was immobilized and all components of the export complex were added. We observed a very low binding of CRM1 to the MBP beads while Nup214 (1916-2033) and $\operatorname{Ran}_{\mathrm{Q} 69 \mathrm{~L}(1-180)} \mathrm{GTP}$ failed to bind, indicating that the export complex was indeed specific (Figure 12a, lane 5).

A

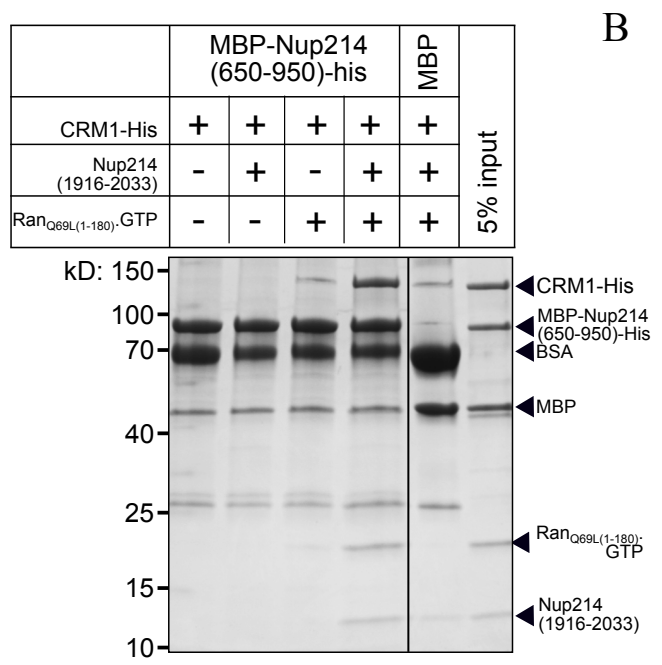

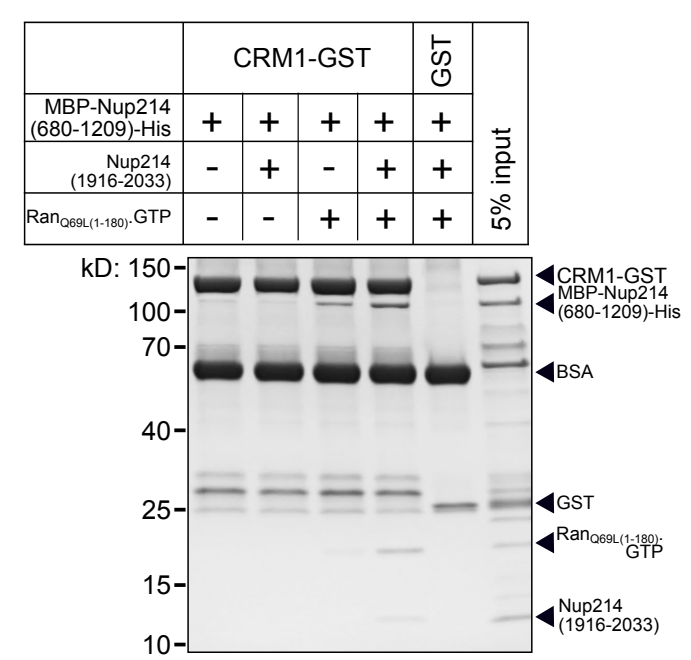


$\mathrm{C}$

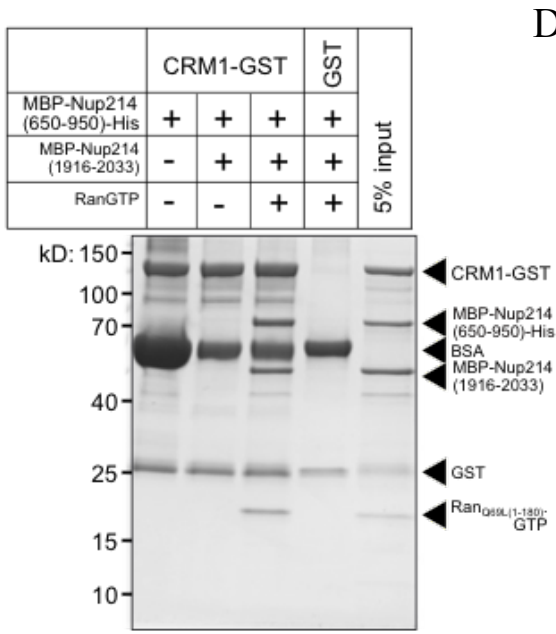

$\mathrm{D}$

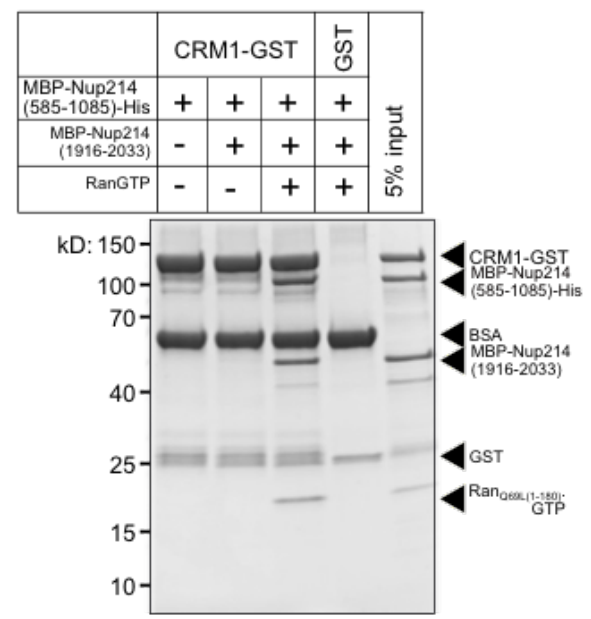

Figure 12: Different Nup214 NES containing fragments can form an export complex in a Ran dependent manner. A single component of the complex was immobilized on beads. The other components of the complex were added in different combinations. In one condition, all components of the complex were added, while in the other conditions at least one of the component was missing. To control the experiment, a single condition was included where only the tag was immobilized. A $5 \%$ input of all recombinant proteins used in each pull down was included. The immobilized protein and the combinations used are shown on top of each pull down. The recombinant protein corresponding to each band on the gel are shown on the righthand side. BSA was used as a blocking reagent. The proteins were separated by SDS-PAGE and stained by commassie brilliant blue. (A) MBP-Nup214 (650-950)-His was immobilized using amylose beads. CRM1-His, Nup214 (1916-2033) and Ran Q69L(1- $_{\text {- }}$ ${ }_{180)}$ GTP were added in different combinations. Immobilized MBP was used as a control. (B-D) CRM1-GST was immobilized using Glutathione Sepharose 4B beads. Either MBP-Nup2 14 (680-1209)-His or MBP-Nup214 (650-950)-His or MBP-Nup214 (585-1085)-His were added in different combinations with Nup214 (1916-2033) and $\operatorname{Ran}_{\mathrm{Q} 69 \mathrm{~L}(1-180)} \mathrm{GTP}$. Immobilized GST was used as a control.

Next, the experiment was reversed by immobilizing CRM1-GST on beads followed by addition of an MBP-Nup214 NES-containing fragment alone or in the presence of either Nup214 (1916$2033)$ or $\operatorname{Ran}_{\mathrm{Q} 69 \mathrm{~L}(1-180)} \mathrm{GTP}$ or both. MBP-Nup214 (680-1209), MBP-Nup214 (650-950) and MBP-Nup214 (585-1085) were used to form the complex. We could form a CRM1 dependent complex in the presence of an MBP-Nup214 NES-containing fragment, Nup214 (1916-2033) and $\operatorname{Ran}_{\mathrm{Q} 69 \mathrm{~L}(1-180)}$ GTP (Figure 12b, lane 4; Figure 12c \& Figure 12d, lane 3). The results confirmed the findings described in the previous paragraph. In presence of Nup214 (19162033), more complex was obtained (Figure $12 \mathrm{~b}$, lane $3 \& 4$ ) while in the absence of $\operatorname{Ran}_{\mathrm{Q} 69 \mathrm{~L}(1-}$ ${ }_{180}$ GTP the complex failed to form (Figure 12b, Figure 12c \&Figure 12d, lane 2). None of the complex components bound to immobilized GST, indicating that the formed complex was specific.

In conclusion, Nup214 interaction with CRM1 is Ran dependent. Even though CRM1, Nup214 NES and RanGTP can form a complex, however, the presence of the FG-repeat region stabilizes the complex. We characterized a direct interaction of Nup214 with CRM1 via its NES in a Ran dependent manner. 


\subsubsection{The Nup214 NES has high affinity for CRM1}

The Nup214 NES region is located between amino acids 875 to 887 of the Nup214 sequence. To confirm that this short putative sequence is a functional NES, plasmids coding for Nup214NES peptide (GST-Nup214 (878-887)) and Nup214-NES peptide mutant (GST-Nup214 (878887)_L878A_L882A_L885A_L887A) were generated and the two peptides were purified. The two GST-tagged peptides were immobilized to see if the NES of Nup214 on its own is capable of forming a complex with CRM1 and $\operatorname{Ran}_{\mathrm{Q} 69 \mathrm{~L}(1-180)} \mathrm{GTP}$ in a similar way as the fragments described in the previous section. Additionally, this experiment aims to see if a mutation in the Nup214 NES sequence will prevent the formation of the complex. As shown in Figure 13a, GST-Nup214 (878-887) was able to form a complex with CRM1 and Nup214 FG-repeat in a Ran dependent manner. By contrast, the mutated version of the peptide failed to form the complex (Figure 13b).

A

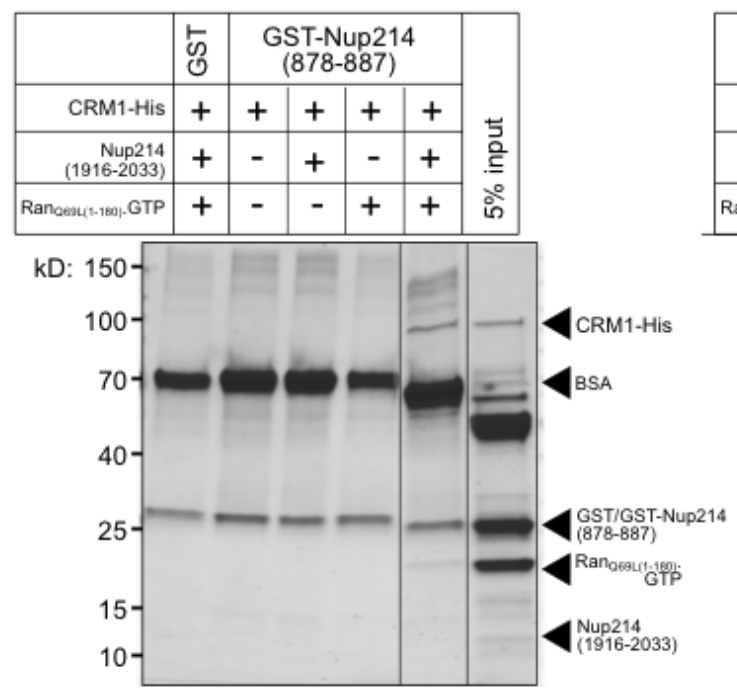

$\mathrm{B}$

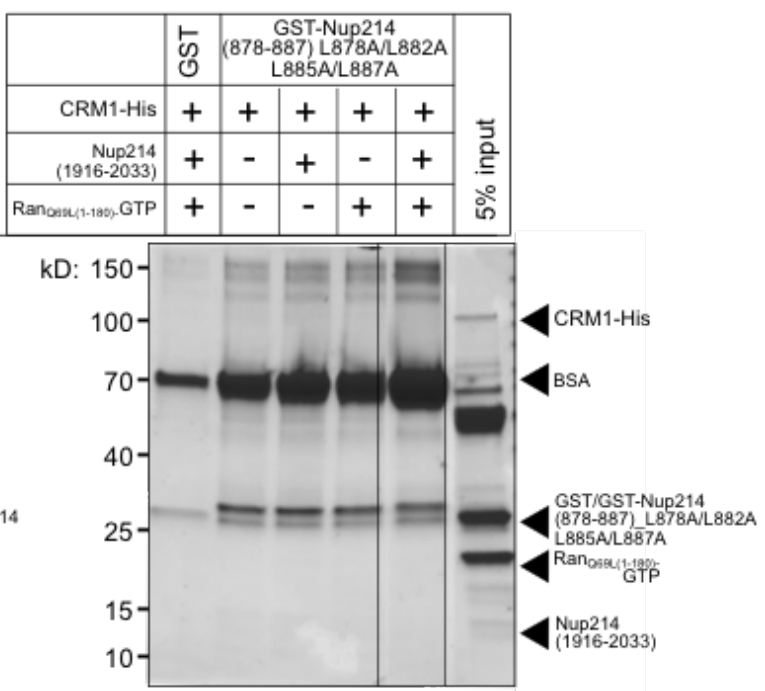

Figure 13: A mutated Nup214 NES peptide fails to form a Ran dependent export complex. GST tagged peptides of Nup214 NES (GST-Nup214 (878-887)) and mutant Nup214 NES (Nup214 (878-887)_L878A_L882A_L885A_L887A) were immobilized on Glutathione Sepharose 4B beads and the different components of the complex were added in various combinations. A single condition had all components of the complex added, while in the other conditions at least one of the component was missing. As a control, GST was immobilized. A 5\% input of all recombinant proteins used in each pull down was included. The immobilized protein and the combinations used are shown on top of each pull down. The recombinant protein corresponding to each band on the gel are shown on the right-hand side. BSA was used as a blocking reagent. The proteins were separated by SDS-PAGE and stained by commassie brilliant blue. (A-B) Either GST-Nup214 (878-887) or GSTNup214 (878-887)_L878A_L882A_L885A_L887A was immobilized using Glutathione Sepharose 4B beads. CRM1-His, Nup2 14 (1916-2033) and $\operatorname{Ran}_{\mathrm{Q} 69 \mathrm{~L}(1-180)}$ GTP were added in different combinations as indicated and immobilized GST was used as a control.

Next, a more quantitative analysis of the $\mathrm{NES}_{\mathrm{Nup} 214}$ peptides was done using fluorescence polarization assay. In this assay, a light source excites a fluorophore in solution. The excited fluorophore will then emit light that will be detected. The degree of polarization of the emitted light depends on the state of the fluorophore. In an unbound state, less polarized light is emitted 
by the fluorophore than when in bound state. This assay can be used to estimate the binding affinity of molecules (Figure 14).

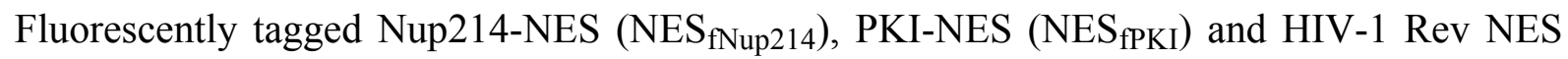
$\left(\mathrm{NES}_{\mathrm{fHIV}-1 \mathrm{Rev}}\right.$ ) were used in this assay (Figure 14a). The binding affinity of $\mathrm{NES}_{\mathrm{fNup} 214}$ was measured in comparison to the previously established peptides, $\mathrm{NES}_{\mathrm{fPKI}}$ and $\mathrm{NES}_{\mathrm{fHIV}-1 \mathrm{Rev}}$.

Fixed concentrations of fNES and $\operatorname{Ran}_{\mathrm{Q} 69 \mathrm{~L}(1-180) \mathrm{GTP}}$ were titrated against increasing concentrations of CRM1. $\mathrm{NES}_{\mathrm{fNup} 214}$ showed a higher affinity to CRM1 in comparison to $\mathrm{NES}_{\mathrm{fPKI}}$ and NES $\mathrm{NHIV}-1 \mathrm{Rev}\left(\mathrm{K}_{\mathrm{D}}: 19 \mathrm{nM}, 81 \mathrm{nM}\right.$ and $499 \mathrm{nM}$ respectively; Figure 15). As a control, the complex was formed in the absence of $\operatorname{Ran}_{\mathrm{Q} 69 \mathrm{~L}(1-180) \mathrm{GTP}}$ and binding did not take place.

In conclusion, a synthesized Nup214 NES peptide binds CRM1 in a Ran dependent manner. Furthermore, its affinity for CRM1 is higher compared to well-established NESs such as the NES of PKI (NES $\mathrm{HIV}-1 \mathrm{Rev}_{\text {) }}$ and the NES of HIV-1 Rev (NES $\left.\mathrm{NIV}-1 \mathrm{Rev}\right)$. 


\section{A}

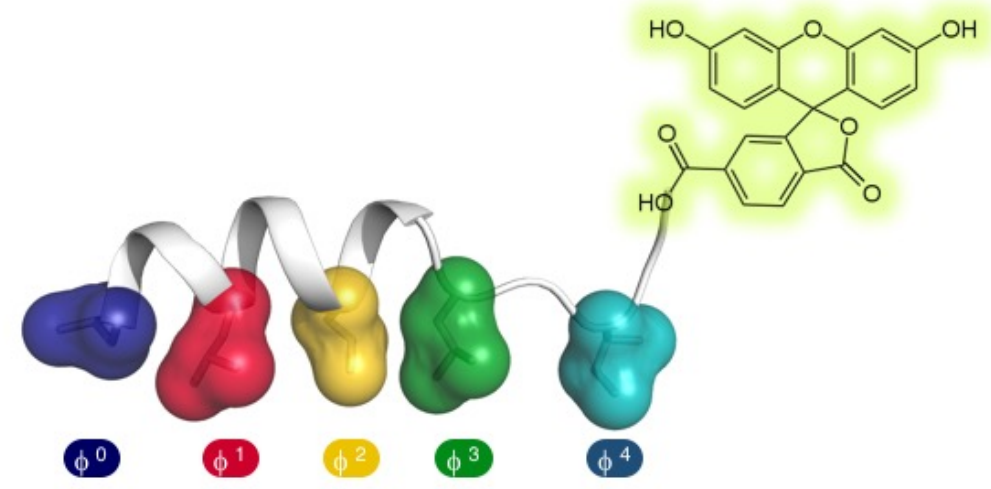
fNup214 NES L N H LVDS L Q Q L R L K
fPKI NES L NE LLAKLA GL D I fHIV1-Rev NES L Q L P P L E R L T L

B

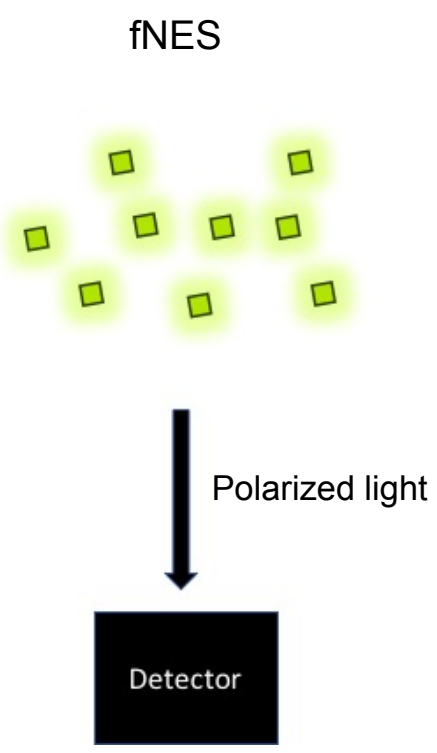

fNES + CRM1 + $\operatorname{Ran}_{\mathrm{Q} 69 \mathrm{~L}(1-180)} \cdot \mathrm{GTP}$
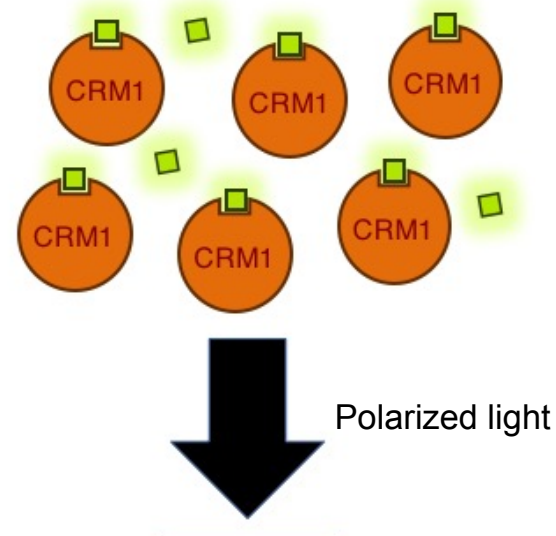

Detector

Figure 14: Concept of the fluorescence polarization assay. Fluorescence polarization or fluorescence anisotropy is used to measure the relative binding affinity of fluorescently tagged peptides, proteins and RNAs. A fluorophore is excited by polarized light and the emission of the fluorophore is detected. The amount of polarized light reaching the detector is used to calculate the fluorescence polarization and hence determine the relative dissociation constant $\left(K_{D}\right)$ of each individual fNES. (A) We used three fluorescently tagged peptides representing the NES regions of three proteins: NES $\mathrm{fNup}_{214}$ (LNHLVDSLQQLRLK), NES $\mathrm{fPKI}_{\mathrm{f}}$ (LNELLAKLAGLDI) and NES $\mathrm{fHIV-1}$ Rev (LQLPPLERLTL). The different

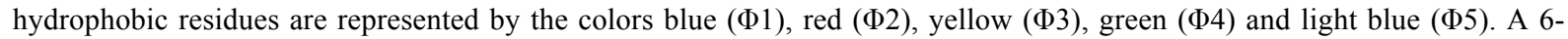
Carboxyfluorescein (6-FAM) was attached to a lysine residue in the NES peptide sequence. (B) fNES on its own emits less polarized light in solution than when in complex with CRM1 and $\operatorname{Ran}_{\mathrm{Q} 69 \mathrm{~L}(1-180)} \mathrm{GTP}$. 


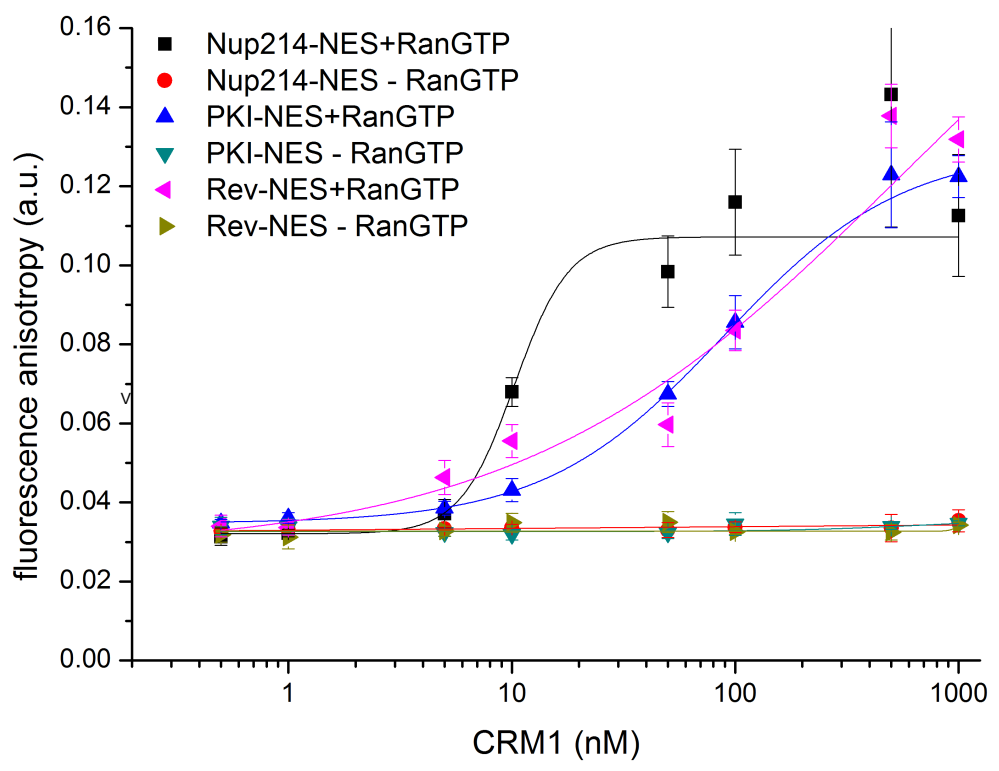

Figure 15: Nup214 NES has a high affinity for CRM1 compared to other NESs. The relative fluorescent anisotropy was plotted as arbitrary units against the concentrations of CRM1 in $\mathrm{nM}$. The mean and standard deviation of three experiments were calculated and plotted as bars. The $\mathrm{K}_{\mathrm{D}}$ values of $\mathrm{NES}_{\mathrm{fNup} 214}, \mathrm{NES}_{\mathrm{fPKI}}$ and $\mathrm{NES}_{\mathrm{fHIV}-1}$ Rev are $19 \mathrm{nM}, 81 \mathrm{nM}$ and $499 \mathrm{nM}$ respectively.

Next, we investigated the indirect affinity of $\mathrm{NES}_{\mathrm{fNup} 214}, \mathrm{NES}_{\mathrm{fPKI}}$ and $\mathrm{NES}_{\mathrm{fHIV}-1 \mathrm{Rev}}$ for CRM1 in the presence of a competitor. First, fixed concentrations of fNES, CRM1 and Ran $\mathrm{Q}_{\mathrm{Q} 9 \mathrm{~L}(1-}$ ${ }_{180)}$ GTP were used to form the complex. Then, the preformed complex was titrated against

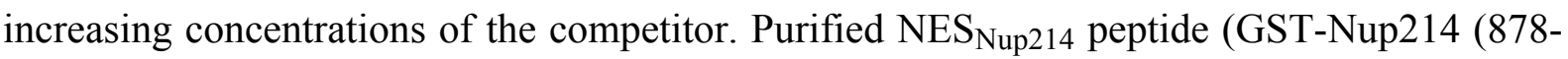
887)) and NES $_{\text {Nup214 }}$ peptide mutant (GST-Nup214 $(878-$ 887)_L878A_L882A_L885A_L887A) were used as competitors. As a control, we used a wellestablished substrate of CRM1 with a very high binding affinity, snurportin 1 (SPN1), as a competitor. In the absence of competitors, the polarization signal was high, indicating a tight binding of the fNES to CRM1 and $\operatorname{Ran}_{\mathrm{Q} 69 \mathrm{~L}(1-180) \mathrm{GTP}}$. At low concentrations of the competitor, the complex was intact. Upon increasing the concentration of GST-Nup214 (878-887), the signal obtained got weaker, indicating that the complex is dessociating. By contrast, the mutated GST-Nup214 (878-887) was unable to compete with the fNES for CRM1, indicating that the observed affinity of the $\mathrm{NES}_{\mathrm{Nup} 214}$ to CRM1 is indeed specific. The use of SPN1 as a competitor resulted in similar effects to that of the purified GST-Nup214 (878-887). However, the concentrations of SPN1 required to release all fNESs from CRM1 binding sites were lower than GST-Nup214 (878-887), i.e. approximately $10 \mathrm{nM}\left(\mathrm{NES}_{\mathrm{fPKI}}\right)$ and $100 \mathrm{nM}$ (for $\mathrm{NES}_{\mathrm{fNup} 214}$ ). This low concentration reflects the fact that SPN1 has a very high affinity for CRM1 (Figure 16). 
fNup214-NES

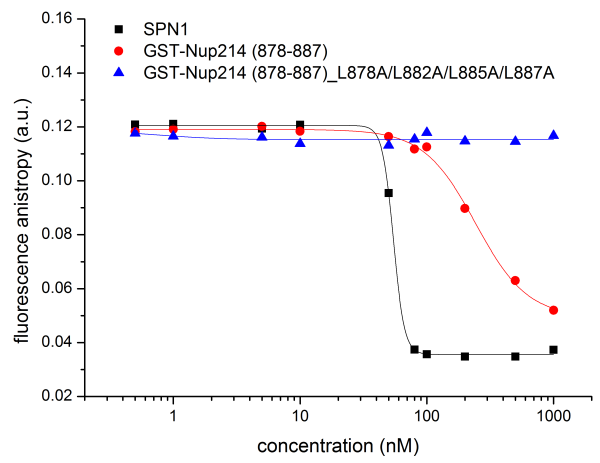

fPKI-NES

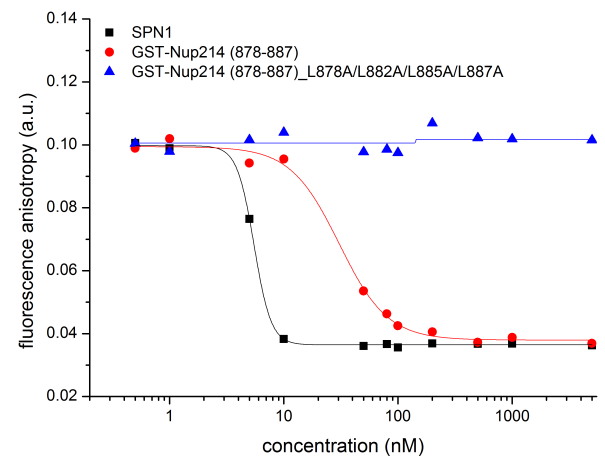

Figure 16: A mutated Nup214 NES peptide cannot compete for CRM1 binding sites. The affinity of fNES for CRM1 can be estimated in the presence of a competitor using fluorescence anisotropy. An fNES-CRM1- $\operatorname{Ran}_{\mathrm{Q} 69 \mathrm{~L}(1-180)}$ GTP complex was formed and then titrated against increasing concentrations of the competitors. Purified snurportin-1 (SPN1), NES Nup214 peptide (GST-Nup214 (878-887)) and NES Nup214 peptide mutant (GST-Nup214 (878-887)_L878A_L882A_L885A_L887A) were used as competitors. The relative fluorescent anisotropy was plotted as arbitrary units against the concentrations of the competitor in $\mathrm{nM}$.

Next, the fluorescently tagged peptides were used to determine the integrity of the formed export complex on gel filtration. Different experimental setups were used including formation of the full complex using all four components or formation of the complex while missing either $\operatorname{Ran}_{\mathrm{Q} 69 \mathrm{~L}(1-180)} \mathrm{GTP}$ or Nup214 FG-repeat fragment, i.e. complex 1: CRM1- Ran ${ }_{\mathrm{Q} 69 \mathrm{~L}(1-180)} \mathrm{GTP}-$

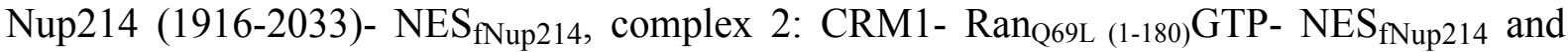
complex 3: CRM1- Nup214 (1916-2033)- NES $\mathrm{fNup}_{214}$. As shown in Figure 17, the complex eluted with a symmetric peak. Analysis of the fluorescent signal indicated that $\mathrm{NES}_{\mathrm{fNup} 214}$ was also part of this complex. In absence of $\operatorname{Ran}_{\mathrm{Q} 69 \mathrm{~L}(1-180)} \mathrm{GTP}$, the complex did not form. In absence of the FG-repeat fragment of the Nup214, less amount of the complex was formed, with 3-5 times less fluorescent signal compared to a complex with all components. The results obtained confirm that a CRM1- Ran ${ }_{\mathrm{Q} 69 \mathrm{~L}(1-180)} \mathrm{GTP}-\mathrm{Nup} 214$ (1916-2033)- $\mathrm{NES}_{\mathrm{fNup} 214}$ complex has the ability to survive the harsh conditions of gel filtration. These results also fit with the results we obtained from the immobilization experiments, where we clearly showed that the complex formation was Ran dependent.

Together, we can conclude that indeed the isolated NES in its own is critical for the formation of the Nup214 export complex, but also in the context of isolated fragments of Nup214, as shown by the immobilization experiments and in the context of the full-length protein as shown in transfected cells. 
Fluorescence analysis of Nup214 export complex (His CRM1 + Nup214 (1916-2033) + Ran1-180Q69L + fNup214-NES)
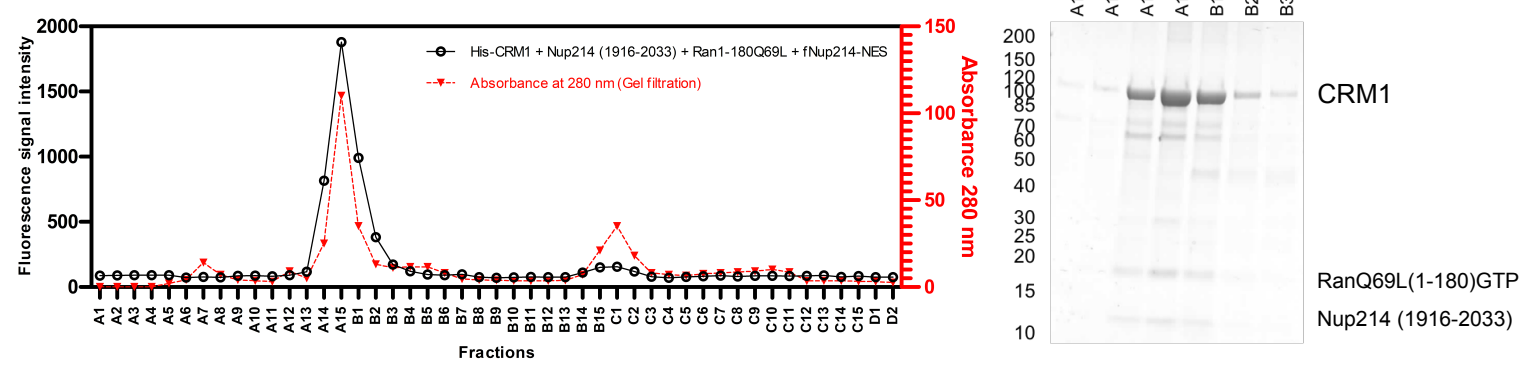

Fluorescence analysis of Nup214 export complex (His CRM1 + Ran1-180Q69L + fNup214-NES)

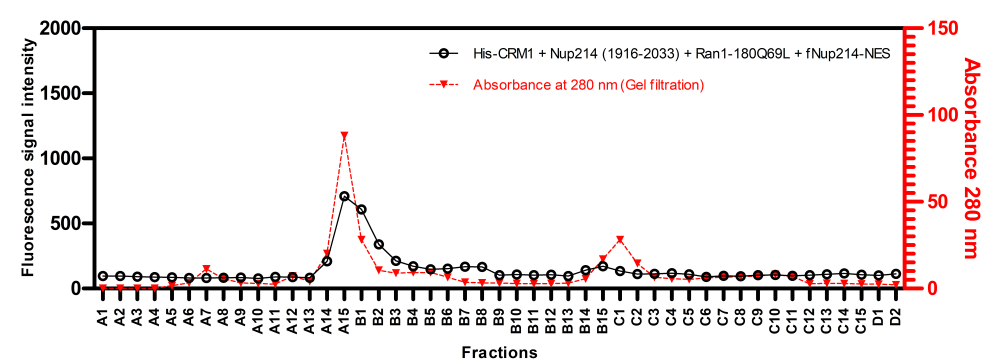

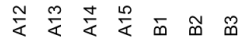

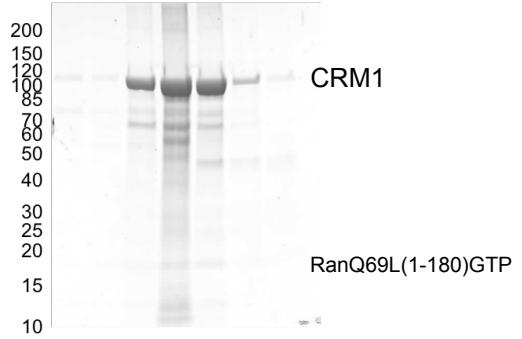

Fluorescence analysis of Nup214 export complex (His CRM1 + Nup214 (1916-2033) + fNup214-NES)
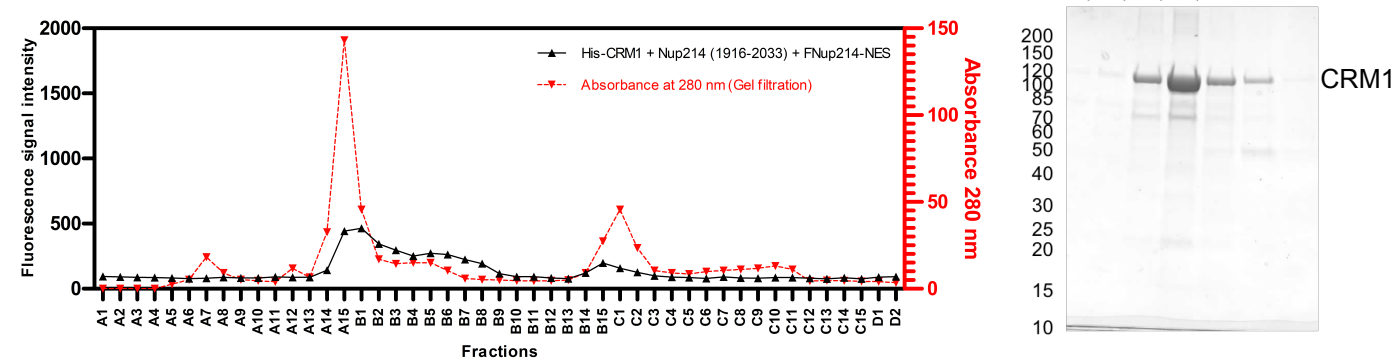

Figure 17: The Nup214 complex is stable on gel filtration. Preformed complexes of fNup214 were analyzed by gel filtration to examine their integrity. In each graph, the absorbance at $280 \mathrm{~nm}$ obtained from the gel filtration chromatogram was plotted in red on the right y-axis, the fluorescence signal was plotted as an arbitrary unit in black on the left y-axis and the fractions eluted from gel filtration are shown on the $\mathrm{x}$-axis. Fractions were picked, separated by SDS-PAGE and stained by commassie brilliant blue as indicated. (A) Analysis of CRM1-Ran ${ }_{\mathrm{Q} 69 \mathrm{~L}(1-180)}$ GTP-Nup214 (1916-2033)- NES $\mathrm{fNup}_{\mathrm{f}} 14$ complex by gel filtration (B) Analysis of CRM1-Ran ${ }_{\mathrm{Q} 69 \mathrm{~L}(1-180)}$ GTP-Nup214 (1916-2033)- NES $\mathrm{fNup} 214$ complex by SDS-PAGE. (C) Analysis of CRM1-Ran ${ }_{\mathrm{Q} 69 \mathrm{~L}(1-180)}$ GTP- NES fNup214 complex by gel filtration. (D) Analysis of CRM1-Ran ${ }_{\mathrm{Q} 69 \mathrm{~L}(1-180)}$ GTP- NES $\mathrm{f}_{\mathrm{fN}} 214$ complex by SDS-PAGE. (E) Analysis of CRM1-Nup214 (1916-2033)- NES $\mathrm{fNup}_{214}$ complex by gel filtration. (F) Analysis of CRM1-Nup214 (1916-2033)- NES $\mathrm{fNup} 214$ complex by SDS-PAGE.

\subsubsection{NES $_{\text {Nup214 }}$ can have a direct effect on Nup88}

Nupp88 has been previously characterized to be the interaction partner of Nup214 (Bastos et al., 1997, Fornerod et al., 1997, Matsuoka et al., 1999, Hutten et al., 2006) and the knockdown of Nup214 resulted in the loss of Nup88 from the NPC (Bernad et al., 2006, Hutten et al., 2006). It is expected that Nup88 and Nup214 can interact through their coiled-coil domain regions 
(Fornerod et al., 1997). The effect of NES $\mathrm{Nup} 214_{\text {on }}$ the localization of Nup88 was investigated. Additionally, we wanted to know if the presence of the $\mathrm{NES}_{\mathrm{Nup} 214}$ has a direct effect on Nup214Nup88 interaction.

A

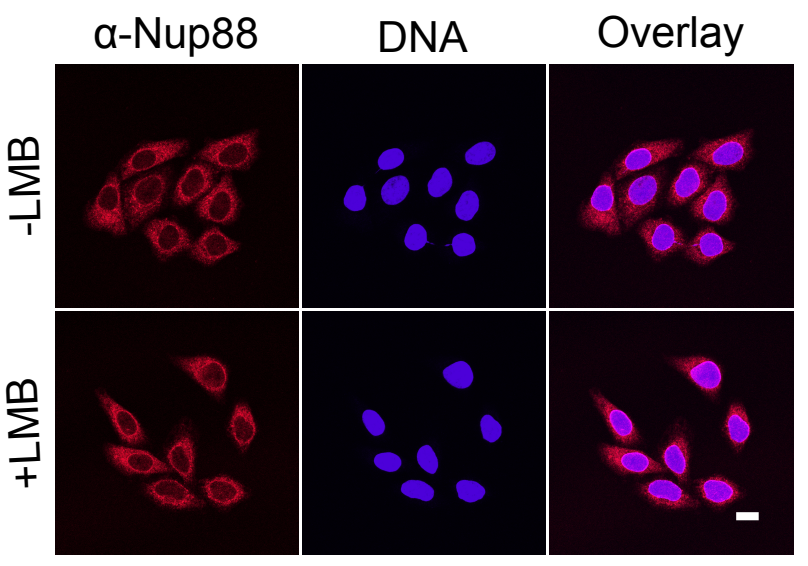

B

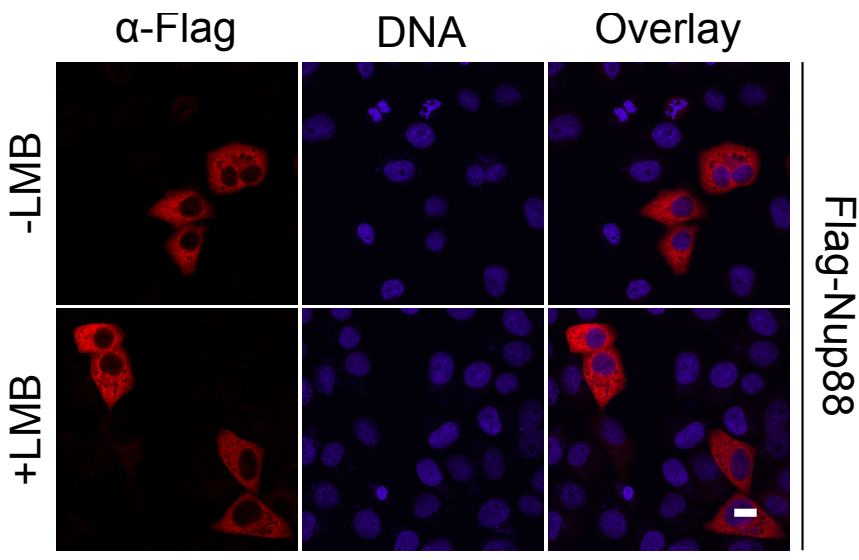

Figure 18: LMB does not affect Nup88 localization. (A) HeLa cells were untreated or treated with $10 \mathrm{nM}$ LMB for 2 hours, as indicated. The cells were immunostained for Nup88 using an anti-Nup88 antibody. Cells were analyzed by confocal microscopy. DNA was stained using DAPI. The scale bars correspond to $20 \mu \mathrm{m}$. (B) Flag-Nup214 was transfected in HeLa P4 cells. The cells were treated with or without $10 \mathrm{nM}$ LMB for 2 hours, as indicated. The cells were immunostained for Flag-tag. The scale bars correspond to $10 \mu \mathrm{m}$.

To address these questions, several in-vivo and in-vitro approaches were used. The interaction of endogenous and overexpressed Nup88 with overexpressed wild type Nup214 and Nup214 with mutation in the NES was investigated. As shown in Figure 18, endogenous and overexpressed Nup88 localize in the cytoplasm and at the NE. We previously investigated the effect of LMB on myc-Nup214 and showed that LMB induced a nuclear localization of mycNup214. To rule out an effect of LMB on Nup88, we tested the effect of LMB on both endogenous and overexpressed Nup88. LMB had no effect on the localization of endogenous (Figure 18a) or overexpressed Nup88 (Figure 18b).

Next, the effect of overexpressing Nup214 on endogenous and exogenous Nup88 was investigated. Upon overexpression of myc-Nup214, endogenous Nup88 co-localized to mycNup214 puncta. FLAG-Nup88 and myc-Nup214 were overexpressed and the presence of 
FLAG-Nup88 in myc-Nup214 puncta was detected (Figure 19). Addition of LMB shifted the localization of endogenous Nup88 to the nuclear compartment, along with myc-Nup214. The results suggested that the localization of Nup88 is affected by the NES region of Nup214. To see if a mutation in this NES would diminish the interaction between Nup88 and Nup214, mycNup214 (L878A/L882A) was overexpressed and stained for endogenous Nup88. In another experimental setup, the myc-Nup214 (L878A/L882A) and FLAG-Nup88 were overexpressed. In both setups, a co-localization of Nup214 and Nup88 was detected, indicating that a mutation in the NES region of the Nup214 does affect the interaction of Nup214 and Nup88 (Figure 20).

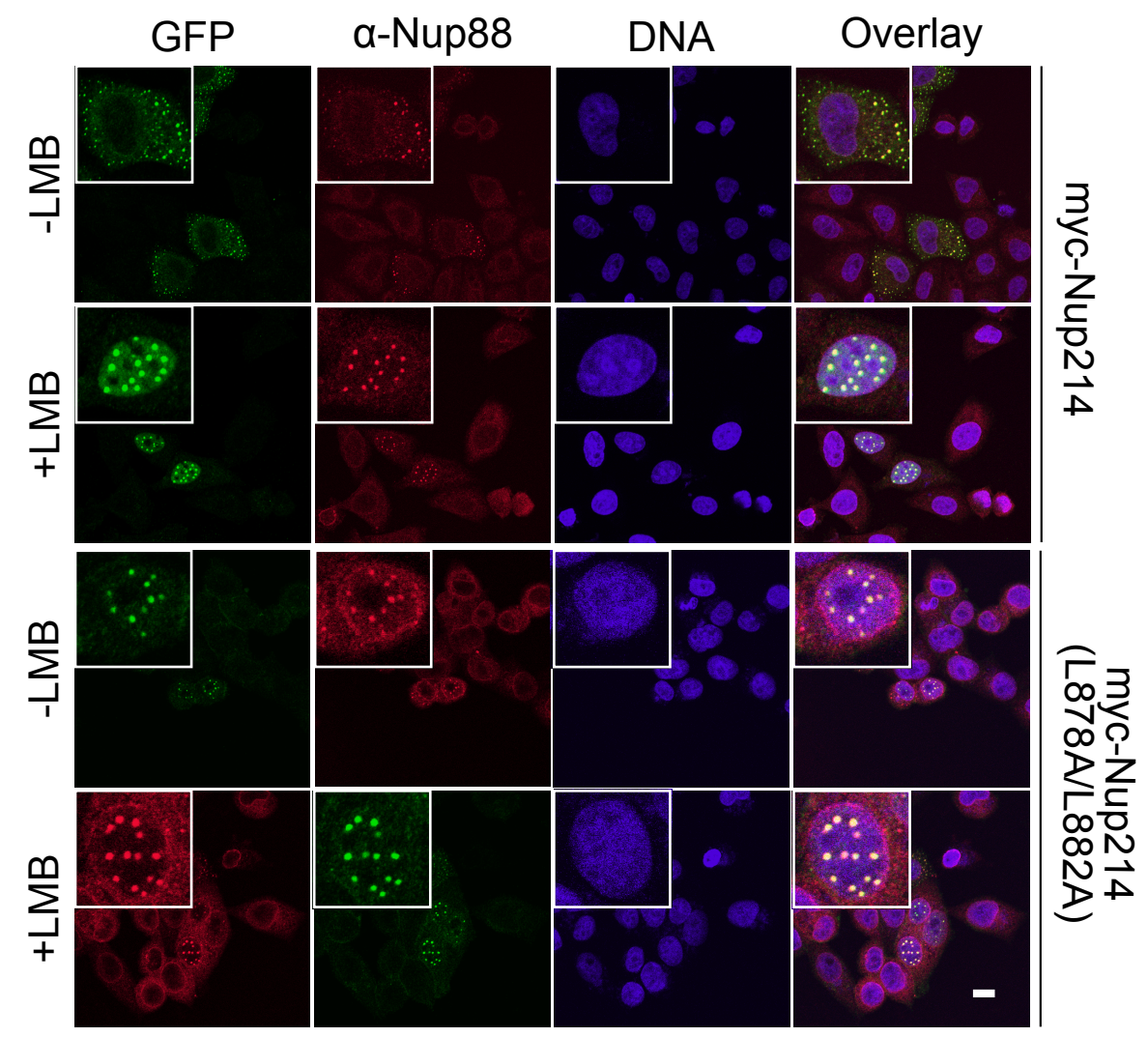

Figure 19: Mutation in the NES region of Nup214 affect the interaction of Nup214 and Nup88. myc-Nup214 and mycNup214 (L878A/L882A) were transfected in HeLa P4 cells. Cells were treated with or without $10 \mathrm{nM} \mathrm{LMB}$ for 2 hours, as indicated. The cells were immunostained for the myc tag and Nup88 using anti-myc and anti-Nup88 antibodies. DNA was stained using DAPI. The scale bar corresponds to $10 \mu \mathrm{m}$.

In conclusion, endogenous and overexpressed of Nup88 co-localize with overexpressed Nup214 in the cytoplasm. The inhibition of Nup214 export by adding LMB or mutating its NES region leads to co-localization of endogenous and overexpressed of Nup88 to the nucleus. 


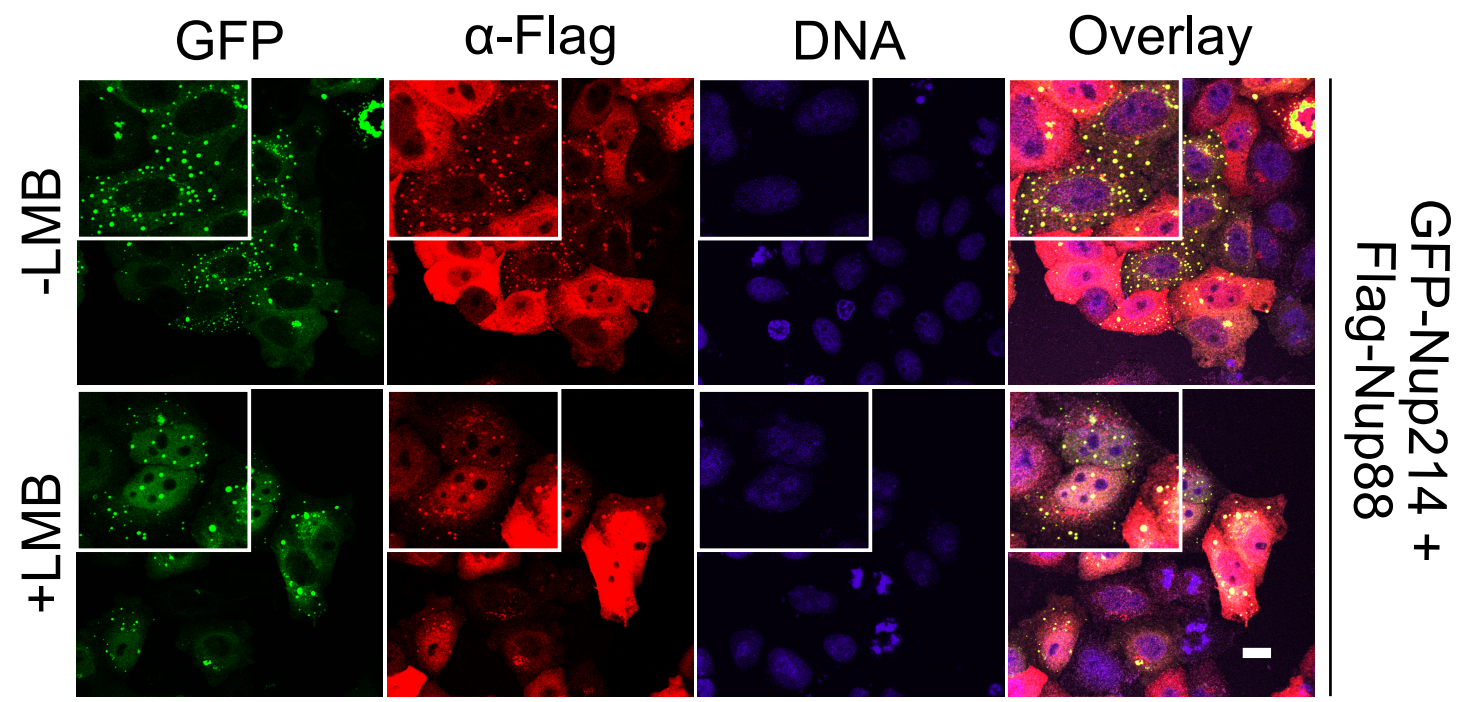

Figure 20: Mutation in the NES region of Nup214 shifts the localization of overexpressed Nup88 to the nucleus. GFPNup214 and Flag_Nup88 were transfected in HeLa P4 cells. Cells were treated with or without $10 \mathrm{nM}$ of LMB for 2 hours, as indicated. The cells were immunostained for the Flag tag using an anti-Flag antibody. DNA was stained using DAPI. All cells were analyzed by confocal microscopy. The scale bar corresponds to $10 \mu \mathrm{m}$.

Furthermore, we investigated the interaction between Nup88 and Nup214 by performing coimmunoprecipitation experiments. Hek293T cells were transfected with plasmids coding for GFP-Nup214 (680-1209) or GFP-Nup214 (680-1209)_L878A/L882A and the proteins were immunoprecipitated using GFP beads. As a control, we transfected a plasmid coding for GFP. As shown in Figure 21, both GFP-Nup214 (680-1209) or GFP-Nup214 (6801209)_L878A/L882A could successfully co-precipitate endogenous Nup88 while GFP alone could not co-precipitate Nup88. These results show that a mutation in Nup214 NES does not abolish the interaction with Nup88.

In another experimental setup, inducible cell lines expressing full length GFP-Nup214 (L878A/L882A) were generated (see Appendix). Co-immunoprecipitation of overexpressed GFP-Nup214 (L878A/L882A) protein and Nup88 was done by immobilizing GFP-Nup214 (L878A/L882A) protein on beads and detecting for the presence of endogenous Nup88. Our results confirm that full length GFP-Nup214 can co-precipitate endogenous Nup88 regardless of the presence of a mutation in the NES region.

By putting these results together, we show that the Nup214 coiled-coil domain fragment interacts with Nup88. In addition, a mutation in the sequence of Nup214 NES did not affect the interaction of this fragment with Nup88 as shown by microscopy and in coimmunoprecipitation experiments. 


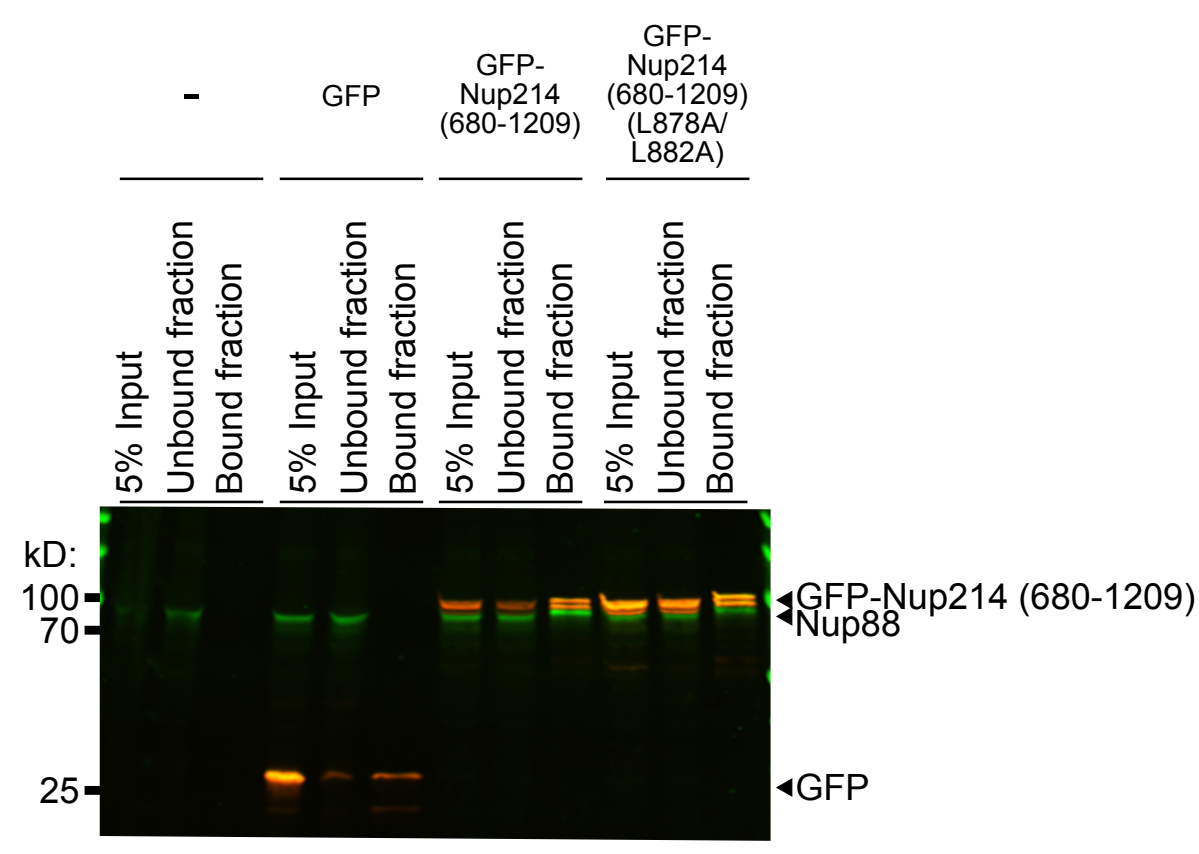

Figure 21: GFP-Nup214 (680-1209) and GFP-Nup214 (680-1209)_L878A/L882A can immunoprecipitate Nup88 from cell lysate. Plasmids coding for GFP, GFP-Nup214 (680-1209) and GFP-Nup214 (680-1209)_L878A_L882A were transfected in Hek293T cells. Non-transfected cells were used as a control. The cells were lysed and the lysate was added to GFP beads. Bound and unbound fractions were analyzed by SDS-PAGE and Western blotting using anti-GFP and anti-Nup88 antibodies.

\subsubsection{Adding an NES sequence to Nup214 mutant can rescue the Nup214 phenotype in cells}

Our experiments demonstrated the presence of an NES within the coiled-coil domain of Nup214. In addition, the NES is necessary for maintaining overexpressed Nup214 in the cytoplasmic compartment of the cell. A question was raised of whether the addition of an NES to another region of a full-length GFP-Nup214 (L878A/L882A) is sufficient to re-localize GFPNup214 (L878A/L882A) back to the cytoplasm. To test this, a NES $\mathrm{Nup} 214_{\text {or }}$ an NES $\mathrm{NIV}_{\text {-1 Rev }}$ was attached to the C-terminus of GFP-Nup214 (L878A/L882A). Cells were transfected with plasmids coding for GFP-Nup214 (L878A/L882A), GFP-Nup214 (L878A/L882A) with the $\mathrm{NES}_{\mathrm{Nup} 214}$ at the C-terminus and GFP-Nup214 (L878A/L882A) with the NES $\mathrm{HIV}_{\text {-1-Rev }}$ at the C-terminus in the presence or absence of LMB. GFP-Nup214 (L878A/L882A) showed a partial localization in the nucleus, while both GFP-Nup214 (L878A/L882A) with the NES $\mathrm{Nup}_{214}$ at the C-terminus and GFP-Nup214 (L878A/L882A) with the $\mathrm{NES}_{\mathrm{HIV}-\mathrm{Rev}}$ at the C-terminus showed a cytoplasmic localization (Figure 22). In addition to its cytoplasmic localization, GFP-Nup214 (L878A/L882A) with $\mathrm{NES}_{\mathrm{Nup} 214}$ at the C-terminus was also localized to the NE. Addition of LMB caused both GFP-Nup214 (L878A/L882A) with Nup214 NES at the C-terminus and GFP-Nup214 (L878A/L882A) with NES HIV-Rev $_{\text {at }}$ the C-terminus to localize to the nucleus, 
indicating that these artificial NESs are functional and CRM1 dependent (Figure 22). In conclusion, adding an artificial NES to the C-terminus of an overexpressed Nup214 with a mutation in the wild type NES resulted in cytoplasmic localization of GFP-Nup214 (L878A/L882A). Furthermore, an artificial Nup214 NES at the C-terminus caused part of the protein to localize to the NE as for the wild type Nup214.

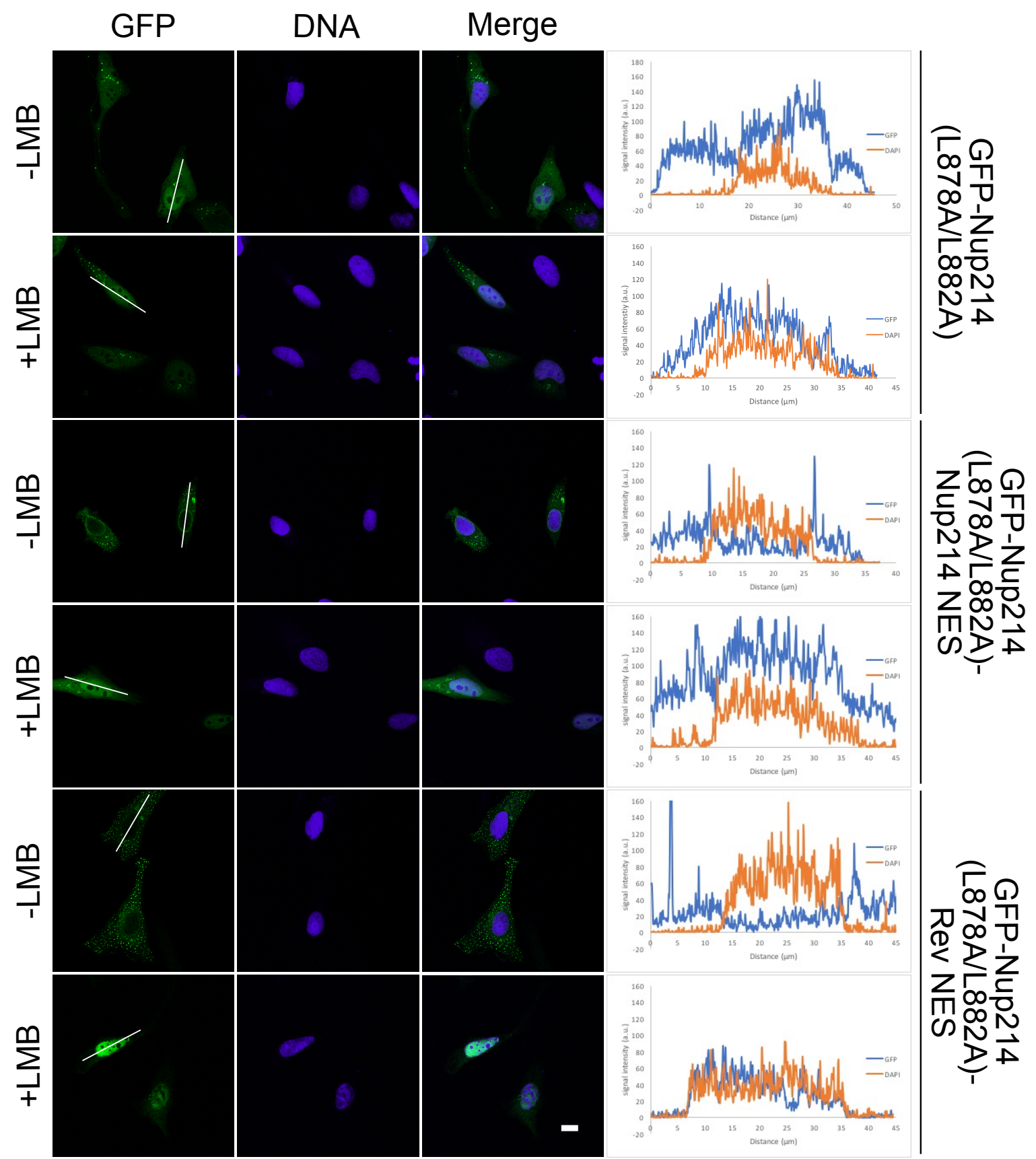

Figure 22: An artificial NES sequence can rescue the phenotype of Nup214 with mutations in the NES. Either the NES of Nup214 or HIV-1 Rev was introduced at the C-terminus end of GFP-Nup214 (L878A/L882A). HeLa P4 cells were transfected with plasmids coding for GFP-Nup214 (L878A/L882A), GFP-Nup214 (L878A/L882A) with Nup214 NES at the C-terminus and GFP-Nup214 (L878A/L882A) with Rev NES at the C-terminus. Cells were treated with or without $10 \mathrm{nM}$ LMB for 2 hours, as indicated. A line was drawn across each cell and the average intensities of five cells from each condition was plotted against the distance of the line on the $\mathrm{x}$-axis shown in um. The scale bar corresponds to $10 \mu \mathrm{m}$. 
To furthermore investigate the effects of adding the artificial NESs, a plasmid coding for GFPNup214 (1916-2033) with a Nup214 NES attached to the C-terminus was constructed. Cells were transfected with the plasmid and were either untreated or treated with LMB. The overexpressed protein largely localized to the cytoplasm and the NE. The addition of LMB resulted in the localization of the protein to the nucleus (Figure 23).

As previously shown in Figure 11, overexpression of the coiled-coil domain fragment GFPNup214 (680-1209) resulted in a cytoplasmic localization but no nuclear rim was observed. On the other hand, transfecting a plasmid coding for GFP-Nup214 (481-1209) resulted in a cytoplasmic and NE localization (Figure 6). These results ensure the functionality of our NESs and additionally point towards a possible function of Nup214 NES in the targeting and insertion of Nup214 at the nuclear envelope with the help of its N-terminal domain.
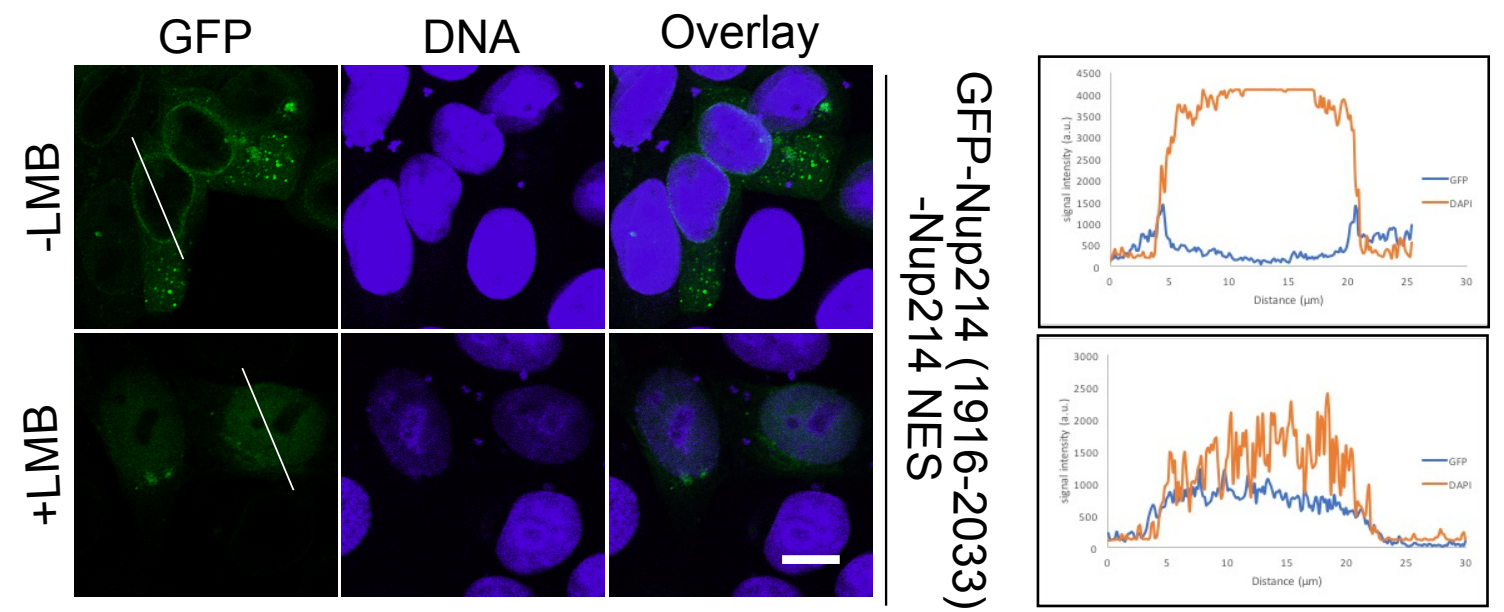

Figure 23: An artificial NES sequence localizes overexpressed GFP-Nup214 (1916-2033) to the cytoplasm. The NES of Nup214 was introduced at the C-terminus of GFP-Nup214 (1916-2033). The plasmid was transfected in HeLa P4 cells. Cells were treated with or without $10 \mathrm{nM} \mathrm{LMB}$ for 2 hours, as indicated. A line was drawn across each cell and the average intensities of five cells from each condition was plotted against the distance of the line on the $\mathrm{x}$-axis shown in um. The scale bar corresponds to $10 \mu \mathrm{m}$.

\subsubsection{Nup358 may play a critical role in helping the insertion of Nup214 into the nuclear envelope}

Nup358 has been characterized in several studies as a docking site for CRM1 recycling and as one of the key proteins of the NPC involved in the release of export complexes (Franke \& Scheer, 1970, Richardson et al., 1988). The effect of Nup358 depletion on Nup214 has been a matter of conflict in previous studies. According to Bernard et al. (2004), the depletion of Nup358 resulted in loss of Nup214. By contrast, Hutten et al. (2006) showed that depletion of Nup358 did not have an effect on Nup214. Previous studies also showed that depletion of Nup214 does not have an effect on Nup358 (Hutten et al., 2006). CRM1, RanGTP and NES containing proteins can form export complexes with high affinity to Nup358 (Engelsma et al., 
2004; Hutten et al.; 2006). The possible involvement of Nup358 in the targeting and/or insertion of Nup214 into the outer nuclear envelope, was investigated. As a first approach, Nup358 was depleted in cells using specific siRNA and either GFP-Nup214 or GFP-Nup214 (L878A/L882A) with NES at the C-terminus, was overexpressed. In control cells and Nup358 depleted cells, GFP-Nup214 and GFP-Nup214 (L878A/L882A) with NES at the C-terminus localized to the cytoplasm. However, Nup358 depletion led to a reduction in the GFP signal at the nuclear rim in Nup358 depleted cells but not in control cells (Figure 24).

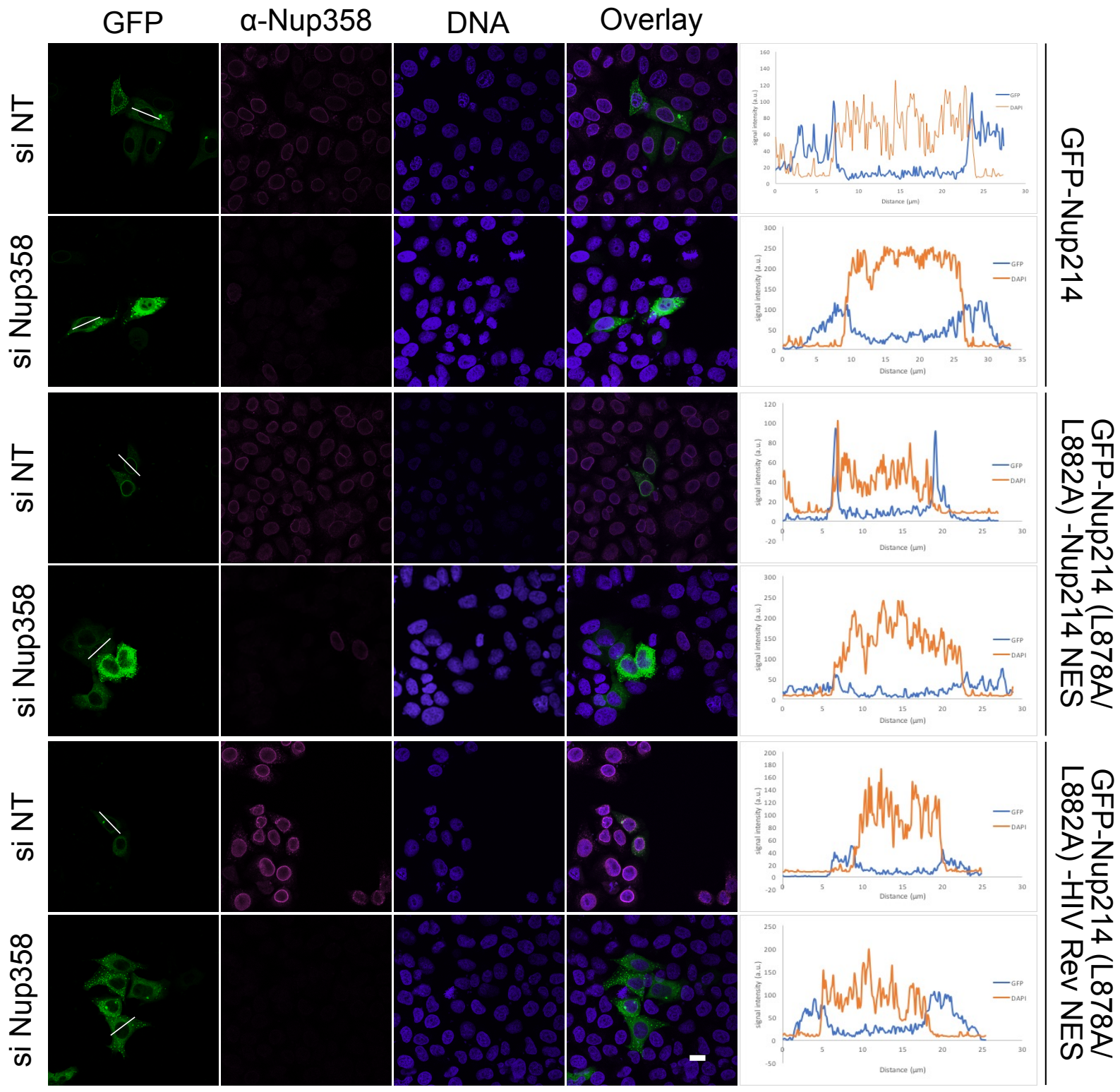

Figure 24: Depletion of Nup358 leads to loss of the Nup214 signal at the nuclear envelope. Nup358 was depleted in HeLa P4 cells by RNA-interference. Plasmids coding for GFP-Nup214, GFP-Nup214 (L878A/L882A) with Nup214 NES at Cterminus and GFP-Nup214 (L878A/L882A) with HIV-1 Rev NES at C-terminus were overexpressed following Nup358 depletion. As a control, the plasmids were overexpressed in cells transfected with non-targeting (NT) siRNA. The cells were immunostained for Nup358. A line was drawn across each cell and the average intensities of five cells from each condition was plotted against the distance of the line on the x-axis shown in um. The scale bar corresponds to $20 \mu \mathrm{m}$. 
The GFP-Nup214 signal at the nuclear envelope could result from the presence of two different populations of GFP-Nup214, either GFP-Nup214 export complex or GFP-Nup214 that got inserted into the NE. To distinguish between the two populations, cells were transfected with plasmids coding for wild type GFP-Nup214 and the GFP-Nup214 (L878A/L882A) with NESs at the C-terminus. Following the transfection, the cell membrane was permeabilized using digitonin and the cytosol was washed away.

+digitonin

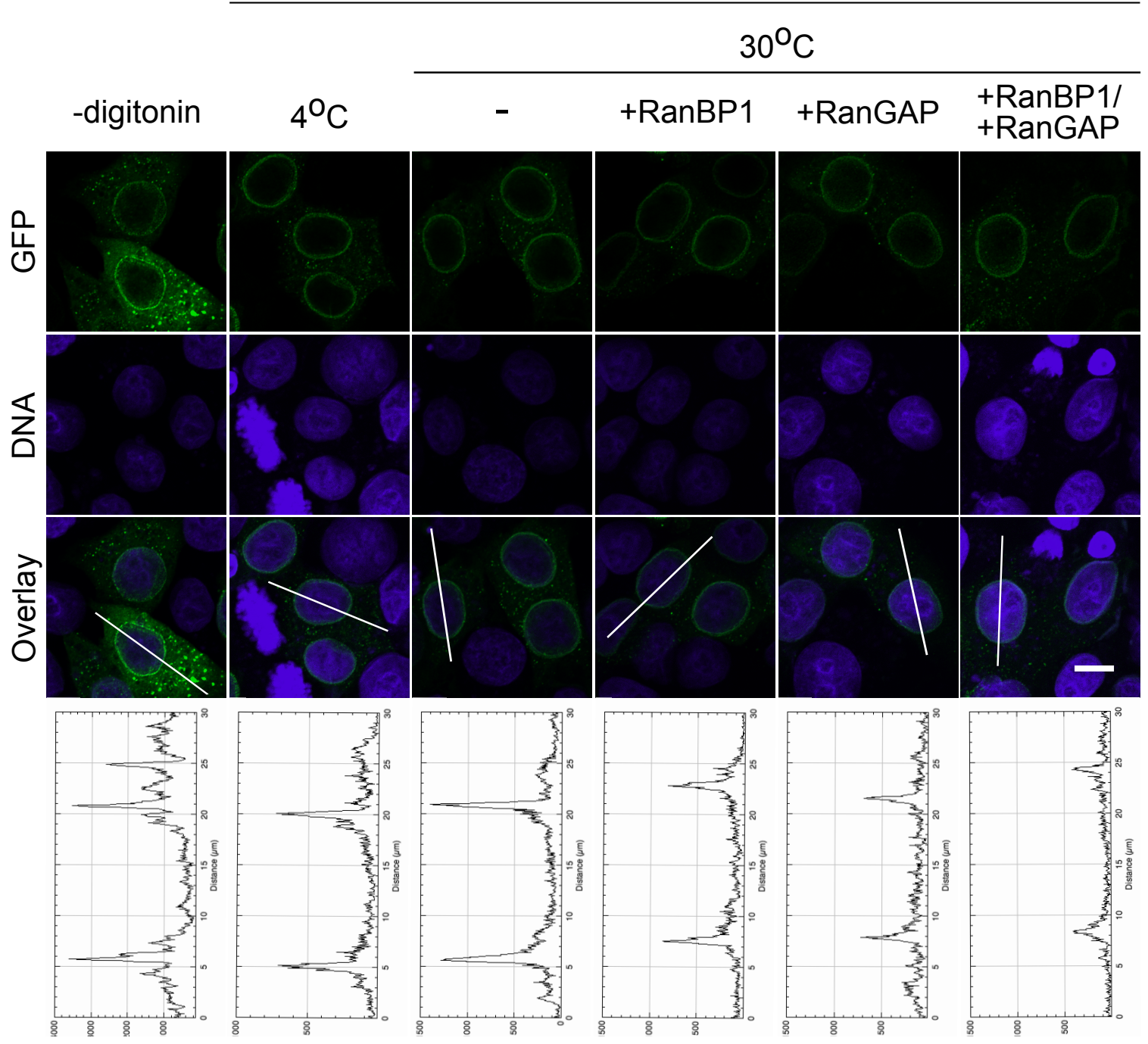

Figure 25: GFP-Nup214 is integrated in the nuclear envelope. HeLa P4 cells were transfected with GFP-Nup214 (L878A/L882A) with Nup214 NES. The cells were permeabilized using digitonin and incubated at $30^{\circ} \mathrm{C}$ with an energyregenerating system and buffer in the absence or presence of either RanBP1 or RanGAP or both, as indicated. As a control, cells were incubated at $4^{\circ} \mathrm{C}$. The GFP signal was viewed under a confocal microscope. The scale bar corresponds to $10 \mu \mathrm{m}$. The GFP signal intensities across the nuclear envelope were measured using ImagaJ software. A line was drawn across each cell and the average intensities of five cells from each condition was plotted against the distance of the line on the $\mathrm{x}$-axis shown in um.

As shown in Figure 25, the digitonin permeabilization of the cells resulted in the loss of the cytoplasmic signal and left a clear NE-signal behind. Previous studies have shown that addition of RanGAP and RanBP1 to permeabilized cells is sufficient to dissociate an export complex (Kehlenbach et al., 1999). To rule out the possibility that what was observed is just 
an export complex not released by washing steps, RanGAP or RanBP1 were included in the reaction. As shown in Figure 25, neither RanGAP nor RanBP1 in their own or together were able to release GFP-Nup214 from the NE. These results indicate that a portion of the GFPNup214 protein transfected was integrated into the NE. Together, our results point to the presence of an inserted population of GFP-Nup214 that got to the NE in not only an NES- but also in a Nup358-dependent manner.

Following an independent approach, proximity ligation assays (PLA) were performed to analyze the interaction of CRM1-Nup214 and CRM1-Nup358. The PLA assay aims at detecting the interaction between two protein in a proximity of $40 \mathrm{~nm}$ from each other. The interaction detected can either be direct or indirect. The PLA is based on using two specific antibodies against the proteins of interest, followed by addition of PLA probes (oligonucleotide-labeled secondary antibodies) that bind to the primary antibodies. Only if the two proteins are in close proximity, the oligos will ligate to each other and a circular closed DNA is formed that serves as a primer for amplification via DNA polymerase (Figure 26a). The amplification reaction produces long repeating complementary oligonucleotide sequence which are finally detected by adding bioconjugate consisting of a fluorescently labeled short oligonucleotide. Each dot seen under the microscope represent a single interaction. To ensure the specificity of the interaction, several controls were included, where either only one of the antibody was used or one of the two interacting protein was depleted by specific siRNAs. Our results revealed CRM1-Nup214 and CRM1-Nup358 interactions, detected as PLA dots at the NE (Figure 26b $\&$ c). These results fit with the pervious biochemistry and microscopy data described earlier. Additionally, knocking down of Nup358, but not Nup214, resulted in a significant decrease in the PLA signals obtained, thus ensuring the specificity of the interaction (Figure 26d). Interestingly, upon knockdown of Nup358, the CRM1-Nup214 signal does not decrease all over the cell but rather decreases at the NE. By contrast, such an effect was not observed with CRM1-Nup358 interaction upon depletion of Nup214. It was shown in previous studies that Nup214 is not lost upon the knockdown of Nup358. These observations showed that Nup214 was not lost upon knockdown of Nup358 but rather localized to the nucleus. For further characterization of the interactions between CRM1-Nup214 and CRM1-Nup358, conditions where cells were incubated with LMB, were included. LMB is known to inhibit the formation of export complexes, however adding LMB did not affect the interaction between CRM1 and Nup214 (Figure 26d). 
a
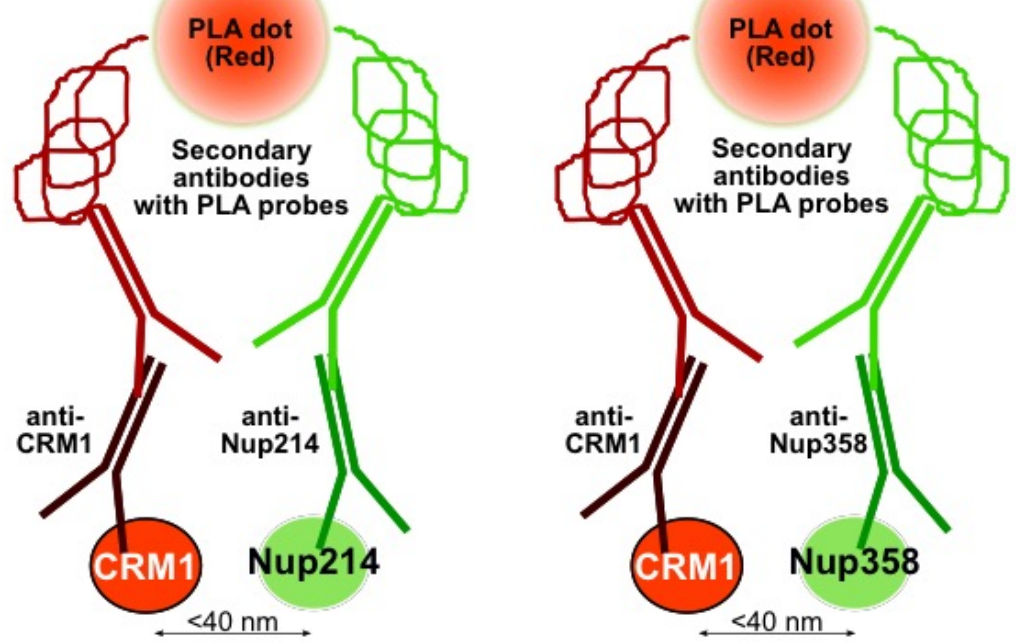

b

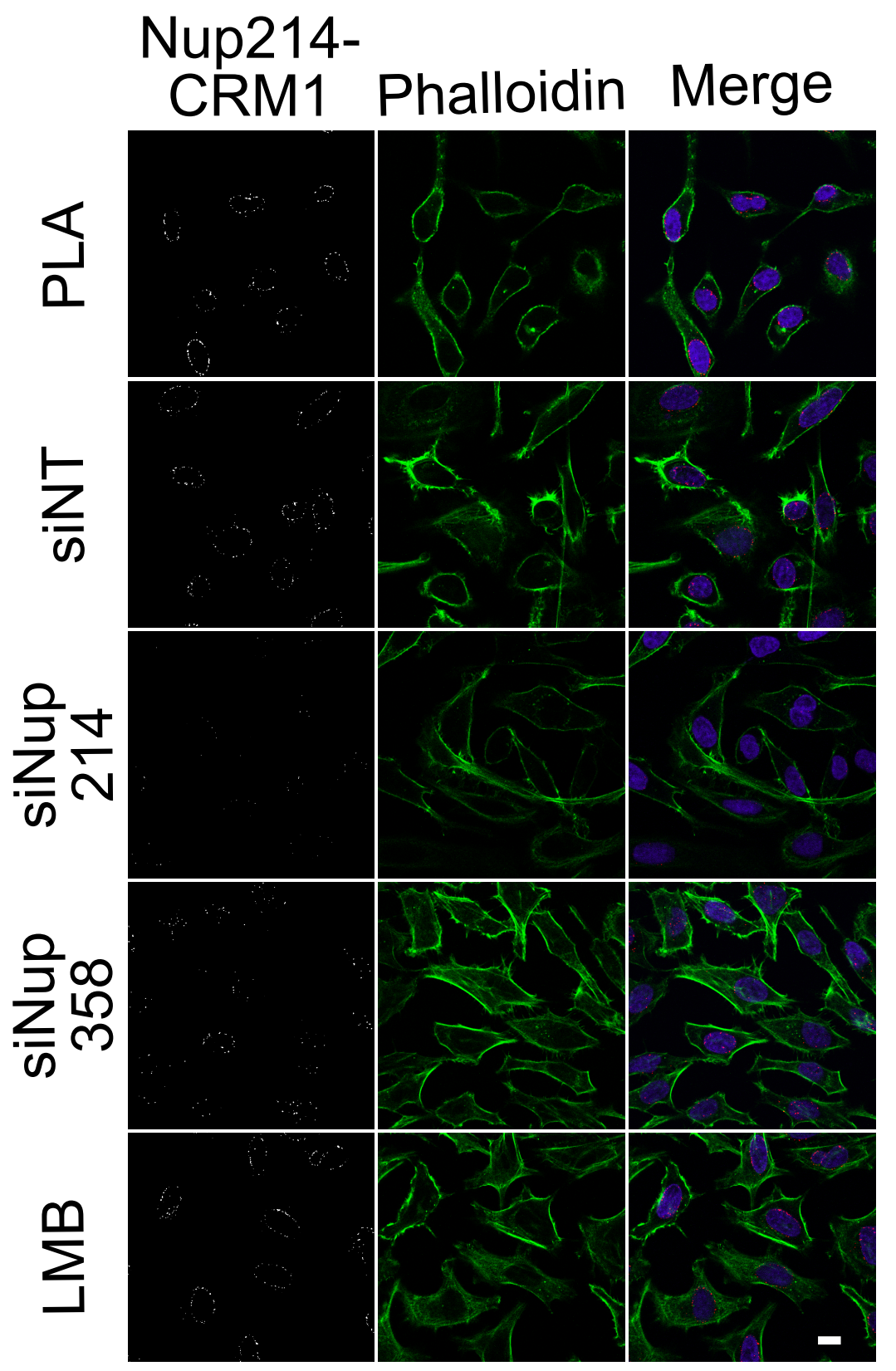


c

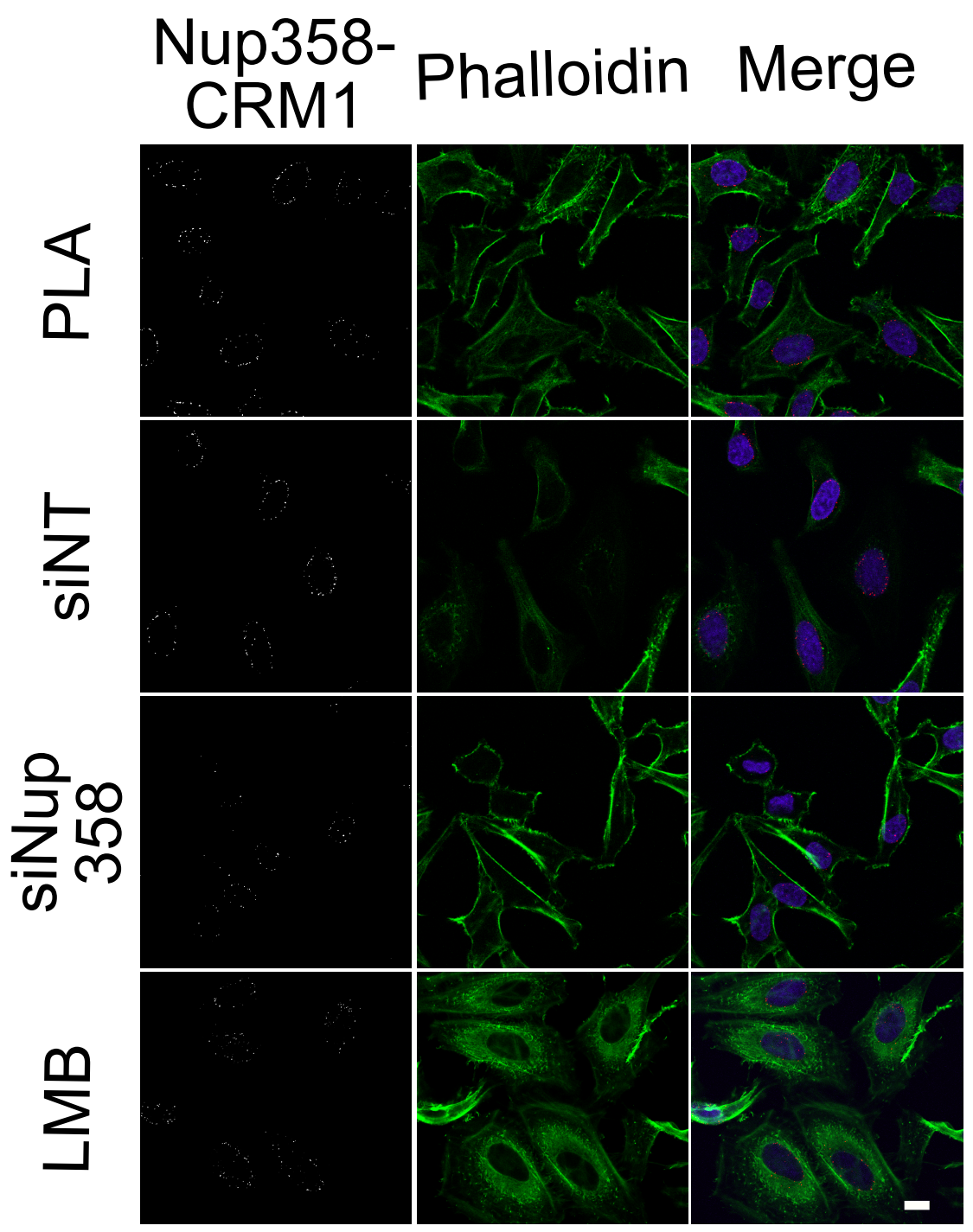

d

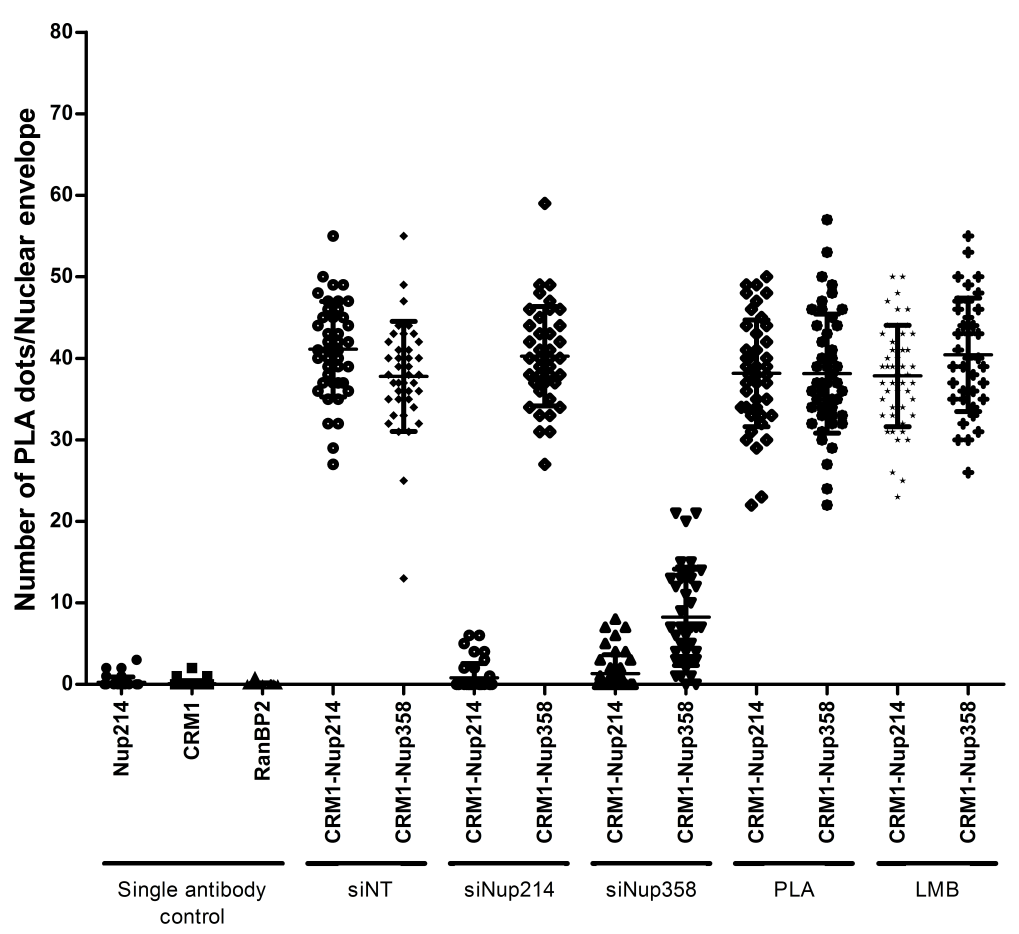


Figure 26: Proximity ligation assays to monitor the proximity of CRM1 and Nup214 or Nup358, respectively. Proximity ligation assays were performed to investigate the interaction of Nup214-CRM1 and Nup358-CRM1 under different conditions. (A) The PLA assay detects the interactions between Nup214 and CRM1 or Nup358 and CRM1. (B-C) Anti-Nup214 and antiCRM1 antibodies or anti-Nup358 and anti-CRM1 antibodies were used to characterize the proximity of Nup214-CRM1 and Nup358-CRM1 in HeLa cells. The PLA was performed in non-transfected cells, Nup214 depleted cells, Nup358 depleted cells and LMB treated cells. Single antibody controls were included where either Nup214 or Nup358 or CRM1 antibody was missing, as indicated. Cells were stained with phalloidin to determine their outline and DNA was stained with DAPI. The scale bar corresponds to $20 \mathrm{um}$. (D) A scatter graph showing the number of PLA dots obtained under different conditions. Each point on the graph represents a single cell. Cells from three independent experiments were counted. The mean and standard deviation were calculated and plotted as indicated.

In conclusion, PLAs shows that CRM1 interacts with both Nup214 and Nup358. However, the close proximity of CRM1, Nup214 and Nup358 might be a reason for obtaining a CRM1Nup358 interaction when detecting for Nup214 and vice versa. Furthermore, depletion of Nup358, but not addition of LMB, resulted in loss of CRM1-Nup214 PLA signal at the nuclear envelope, suggesting that Nup358 can play a role in Nup214 insertion at the NE.

\subsubsection{Overexpression of Nup214 can restore Nup88 localization in Nup214 depleted cells}

Previous studies showed that Nup88 and Nup214 are mutual interacting partners (Bastos et al., 1997). It was also shown in other studies that depletion of Nup214 leads to complete loss of Nup88 (Fornerod et al., 1997). To investigate the effect of Nup214 depletion on Nup88, Nup214 was depleted from cells using a specific siRNA followed by transfection of plasmids coding for GFP-Nup214 or GFP-Nup214 (L878A/L882A) with Nup214 NES at the C-terminus. As a control, plasmids coding for GFP-Nup214 or GFP-Nup214 (L878A/L882A) with Nup214 NES at the C-terminus were transfected in cells treated with non-targeting siRNA. As shown in Figure 27, depletion of endogenous Nup214 led to a decrease in Nup88 signal. Overexpression of exogenous Nup214 following the depletion of endogenous Nup214 resulted in re-expression of endogenous Nup88 in a Nup214 dependent manner. Additionally, the GFP signal was detected mainly, but not always exclusively, at the NE of transfected cells. A possible explanation for this is that depletion of endogenous Nup214 caused binding sites to become available for transfected Nup214 to accumulate at the NE. 

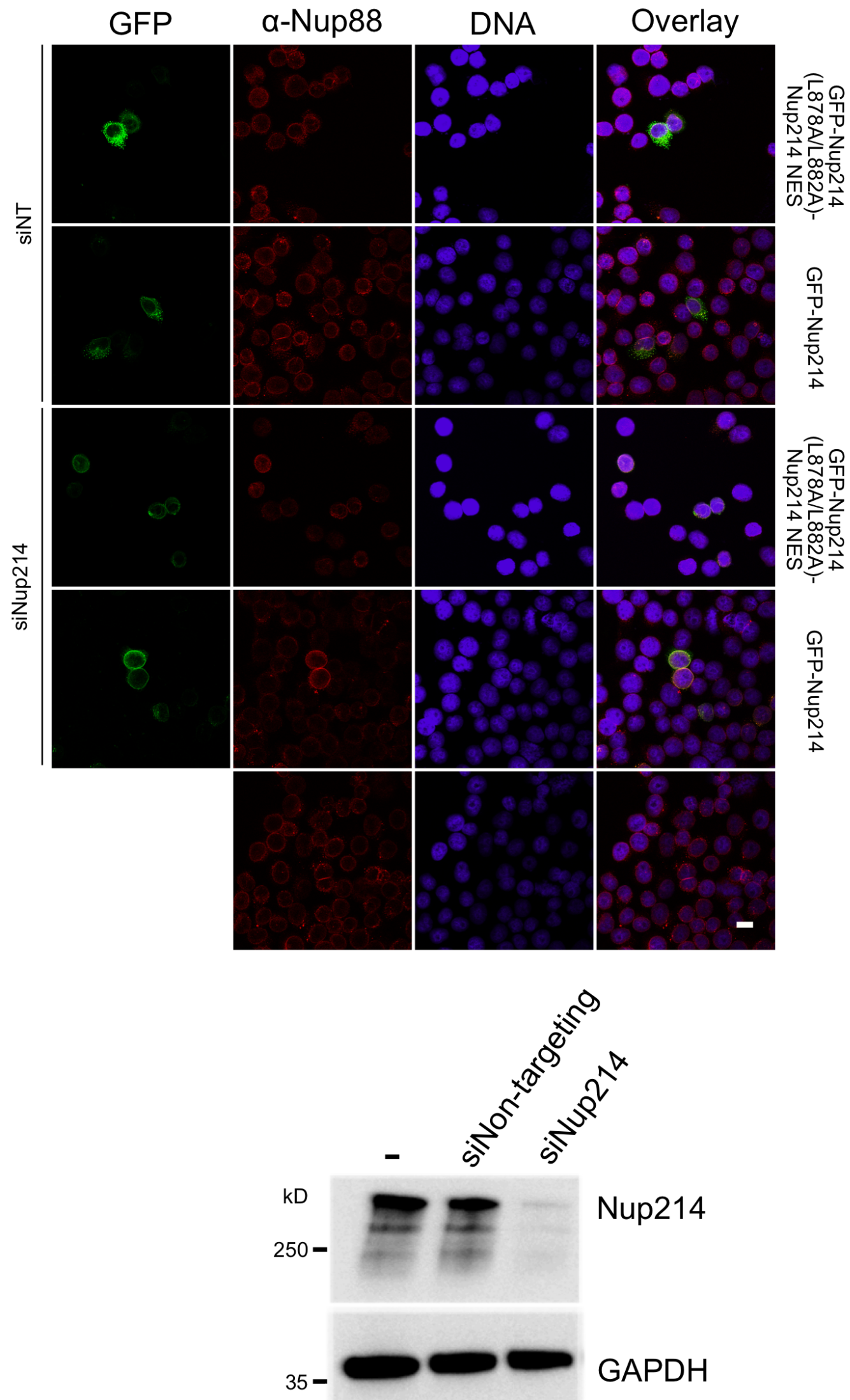

Figure 27: Overexpression of Nup214 restores Nup88 in Nup214 depleted cells. Plasmids coding for GFP-Nup214 and GFP-Nup214 (L878A/L882A) with the Nup214 NES at the C-terminus were transfected in HeLa P4 cells treated with nontargeting siRNA or siRNA against Nup214. The cells were immunostained for Nup88. The scale bar corresponds to $10 \mu \mathrm{m}$. To test for the knockdown efficiency of Nup214, lysate from Nup214-depleted cells or control cells were anaylsed by SDSPAGE and Western blotting, detecting Nup214. GAPDH was used as a loading control. 


\section{Discussion}

The role of Nup214 in nucleocytoplasmic transport has been under investigation over the past decades. Several studies (Moroianu et. al., 1995, Van-Deursen et. al., 1996, Napetschnig et al., 2007) have reported the importance of Nup214 in different functions including the regulation of CRM1 dependent export of some cargos. The depletion of Nup214, or it yeast homolog Nup159, had a wide spectrum of negative effects including defects in nucleocytoplasmic transport and cell death in human cells (Boer et al., 1998), early embryonic death in animal models (Van-Deursen et. al., 1996) and nucleocytoplasmic transport defects in yeast (Rout \& Wente, 1994). The Nup214 interaction with CRM1 has been reported in several studies (Fornerod et al., 1997; Kehlenbach et al., 1999; Roloff et al., 2013; Port et al., 2015).

Addition of LMB to cells overexpressing exogenous Nup214 shifted Nup214 localization from the cytoplasm to the nucleus. Scanning Nup214 for an NES revealed a putative sequence within the coiled-coil domain of Nup214 which is conserved in vertebrates. In light of this finding, several questions were raised. First, if that NES is a characteristic of CRM1 export cargo, would Nup214 interact with CRM1 in a RanGTP dependent manner? Second, different NESs have different binding affinities to CRM1, so how strong is Nup214 NES affinity for CRM1 when compared to other NESs? Does Nup214 NES has a function in termination of export complexes? Would the presence of the NES in the coiled-coil domain affect the interaction between Nup214 and Nup88? Finally, how could this NES contribute to the localization and/or targeting of Nup214 to the NPC?

To answer the previous questions, biochemical and cell biological techniques were used to analyze the interaction between Nup214 NES and CRM1. The affinity of Nup214 NES to CRM1 was measured in comparison to published NESs. Furthermore, the effect the Nup214 NES has on the localization of its mutual partner, Nup88, was studied. Finally, RNAi experiments were used to get an insight in the role of Nup214 NES in targeting and/or incorporation of Nup214 into the NPC and possible models of Nup214 NES function were generated.

\subsection{Different regions of Nup214 show differential localization patterns}

Previous reports showed that endogenous and overexpressed full length Nup214 localize at the cytoplasm and the NE (Figure 5, Shah et al., 1998, Hutten et al., 2006). However, when expressed on their own, different regions of Nup214 have different localization patterns. Fragments of FG-repeat domain (aa 1859-2090) localize to the nucleus suggesting that they 
interact with the FG-barrier of the central channel of the NPC (Figure 6). Furthermore, the coiled-coil domain (aa 680-1209) localizes in the cytoplasm (Figure 11). An additional Nterminal sequence (aa 451-1209) localizes the coiled-coil domain to the cytoplasm, but also the NE (Figure 6). Previous reports have postulated the role of the N-terminus of Nup214 in nuclear pore assembly (Napetschnig et al., 2007).

In conclusion, full length Nup214 has different domains that contribute to its localization. These domains might change their conformation to interact with surrounding proteins according to the location of Nup214 within the cell and hence affect the function of Nup214. The different domains of Nup214 may play a role in the biogenesis of Nup214 within the cell from the moment it is translated until it is incorporated into the NPC. So far, the role of each domain has not been characterized. However, separate studies connected Nup214 domains to cell cycle events (Napetschnig et al., 2007), export of complexes (van-Deursen et al., 1996) and recruitment of the RNA machinery (Montpetit et al., 2011).

\subsection{Nup214 binds CRM1 in a RanGTP dependent manner}

The interaction of Nup214 and CRM1 has long been studied and reported to be conserved across different organisms as vertebrates (Port et al, 2015), yeast (Allen et al., 2002) and drosophila (Xylourgidis et al., 2006). In particular, Nup214 FG-repeats have been well characterized biochemically and structurally. It was shown that Nup214 FG-repeats play a role in stabilizing the export complexes of CRM1, suggesting a role of Nup214 in nuclear export (Port et al., 2015). Several export complexes have been formed and characterized such as CRM1-RanGTP-SPN1 (Dong et al, 2009, Monecke et al., 2009), CRM1-RanGTP-PKI (Wen et al, 1995), CRM1-RanGTP-HIV-1 Rev (Fischer et al, 1995). A feature of all export complexes is that they require RanGTP to be formed.

The presence of an NES sequence has neither been reported in Nup214 nor any of the other Nups at the cytoplasmic face of the NPC. Having such a conserved sequence in Nup214 suggests that it may have an important role in evolution. The reported consensus of an NES sequence is $\Phi 0-X-\Phi 1-(\mathrm{X})_{2-3}-\Phi 2-(\mathrm{X})_{2-3}-\Phi 3-\mathrm{X}-\Phi 4$ (Güttler et al, 2011). Indeed, Nup214 NES sequence (LNHLVDSLQQLRL) follows this consensus where $\Phi$ is leucine.

LMB is a prominent inhibitor of CRM1 export (Hamamoto et al., 1983, Adachi \& Yanagida, 1989). It covalently modifies the NES binding cleft of CRM1, thus inhibiting nuclear export. LMB has been used to study the effect of inhibiting the export of different CRM1 cargos. Addition of LMB to cells overexpressing Nup214 resulted in nuclear accumulation of Nup214. This behavior is unique to CRM1 substrates that get exported in a RanGTP dependent manner. 
However, LMB does not have an effect on endogenous Nup214. A possible explanation is that endogenous Nup214 is mostly incorporated at the NE, and hence does not form a complex with CRM1 and RanGTP. Mutating two leucine residues in the NES sequence of Nup214 resulted in a significant increase of Nup214 accumulation in the nucleus. Similar to the Nup153 NLS that was previously described for its importance in maintaining Nup153 at the nuclear side of the NPC (Nakielny et al., 1999, Daigle et al., 2001), our observations suggest that NES Nup214 might play a similar role in maintaining Nup214 in the cytoplasm or on the cytoplasmic side of the NPC. Furthermore, it might contribute to the disassembly of export complexes at the cytoplasmic face of the NPC. Overexpressed Nup214 FG-repeat fragments showed a nuclear localization, hence, the presence of an NES in Nup214 can be important to maintain the cytoplasmic localization of Nup214.

Moreover, a purified fragment of the Nup214 coiled-coil domain could form an export complex in the presence of RanGTP. The addition of a purified FG-repeat fragment resulted in the formation of a more stable export complex, fitting with previous reports showing that Nup214 FG-repeat increase the stability of export complexes (Port et al, 2015).

In conclusion, Nup214 has an NES located within the coiled coil domain and binds CRM1 in a RanGTP dependent manner. Inhibition of CRM1 mediated export by LMB or mutating the NES, led to nuclear localization of overexpressed Nup214. Additionally, the FG-repeat domain of Nup214 increased the stability of the complex obtained indicating that it can play a role in increasing the stability of the Nup214 export complex.

\subsection{The NES of Nup214 has a high affinity for CRM1}

The correlation between binding affinity of CRM1 to different NESs and nuclear export has long been studied. Several reports showed that CRM1 can bind various NESs with $\mathrm{K}_{d} \mathrm{~S}$ in the nanomolar to micromolar range.

Using fluorescent polarization assays, the binding affinity of the NES of Nup214 was analyzed compared to the published NESs of protein kinase inhibitor protein (PKI) and the HIV-1 Rev protein. The measured $\mathrm{K}_{d}$ values of Nup214-, PKI- and HIV-1 REV- NESs were $19 \mathrm{nM}, 81 \mathrm{nM}$ and $500 \mathrm{nM}$ respectively. The $\mathrm{K}_{\mathrm{d}}$ values of PKI-NES and HIV-1 Rev NESs are similar to previously published values showing that PKI NES has a high affinity of $34 \mathrm{nM}$ and HIV-1 Rev NES has a low affinity of $1180 \mathrm{nM}$ (Fu et al., 2018). Nup214 NES shows a high affinity to CRM1 among all other previously studied NESs. According to Fu et al. (2018), the $\mathrm{K}_{\mathrm{d}}$ of Nup214 falls within the limit for optimal CRM1-NES affinity which ranges between 4 and 34 nM. However, it is not clear why Nup214 NES has a higher affinity for CRM1 than other NESs. 
A possible explanation is that Nup214 NES competes with the NESs of certain export cargos such as NFAT (Kehlenbach et al., 1998) and the S60 ribosomal subunit (Bernad et al., 2006) to release them from CRM1 at the cytoplasmic side of the NPC. Indeed, a low concentration of purified Nup214 NES peptide was able to release the PKI NES peptide from CRM1 (Figure 16). A Similar function was suggested for Nup98, where an identified NLS sequence in Nup98 was shown to play a role in disassembly of Kap- $\beta 2$ import complexes through competition with Kap- $\beta 2$ imported cargo NLS (Fontoura et al., 2000).

In conclusion, we show that Nup214 NES possesses one of the highest binding affinities for CRM1 among all published NESs.

\subsection{The Nup88-Nup214 subcomplex localizes within the cell in an NES dependent manner}

Nup88 exists in a complex with Nup214 at the cytoplasmic face of the NPC. Studies showed an interdependence of Nup88 and Nup214, where Nup214 depletion leads to loss of Nup88 (Bastos et al., 1997, Fornerod et al., 1997, Hutten et al., 2006). In fact, Nup88 acts as a bridge between Nup214 and the cytoplasmic face of the NPC. The targeting of Nup88 and Nup214 to the NE may also require the presence of the two proteins in a subcomplex (Bernad et al., 2004). Our observations showed that endogenous and exogenous Nup88 localized to Nup214 puncta in the cytoplasm in overexpression experiments (Figure 19 \& Figure 20). Addition of LMB or mutation of the NES sequence of Nup214 resulted in localization of overexpressed Nup214 to the nucleus, but also localization of its binding partner Nup88. Previous reports (Fornerod et al., 1997) showed that Nup214 and Nup88 interact via their coiled-coil domains. In addition, Fornerod et al. (1996) showed that the interaction occurs via a valine residue within the NES region identified in this thesis. The presence of an NES within the coiled-coil domain of Nup214 was speculated to affect the interaction with Nup88. The question was raised of whether a mutation in the NES of Nup214 can affect the interaction with Nup88. Coimmunoprecipitation experiments showed that an overexpressed coiled-coil domain of wild type Nup214 co-precipitated Nup88. A Mutation in the NES sequence of either overexpressed full length or a coiled-coil domain fragment did not cease the interaction of Nup214 with Nup88. Moreover, RNAi experiments showed that depletion of Nup214 resulted in codepletion of Nup88 (Fornerod et al., 1997, Bernad et al., 2006, Hutten et al., 2006). However, overexpression of exogenous Nup214 rescued endogenous Nup88 and the resulting subcomplex localized at the NE. Biophysical, biochemical and EM data showed that Nup159 (Nup214 homolog), Nup82 (Nup88 homolog) and Nsp1 (Nup62 homolog) in Chaetomium 
thermophilum interact via their coiled-coil domain and that their coiled-coil domains contribute to the asymmetric localization of the three Nups at the cytoplasmic face of the NPC (Gaik et al., 2015). A similar trimer was described between Nup159 (the Nup214 homolog in yeast), Nup82 (Nup88 homolog) and Nup116, where Nup82 and Nup159 interact via their coiled-coil domains (Yoshida et al., 2011).

These findings prove that Nup88 and Nup214 are interdependent. The organization of Nup88 and Nup214 in relation to other nucleoporins of the NPC is not well studied. However, one possible explanation for this interdependence is that Nup88 binding is necessary to change the conformation of Nup214 to expose the NES of Nup214 for CRM1 binding.

In conclusion, Nup214 and Nup88 are interdependent and depletion of Nup214 led to the codepletion of Nup88. The importance of this interdependence is not clear; however, it was postulated that it might play a role in targeting of Nup214 to the NPC via for the NES of Nup214.

\subsection{An artificial NES sequence at the C-terminus of Nup214 could form a CRM1 dependent export complex}

Exogenous Nup214 with mutations in the NES partially localized to the nucleus. Published studies showed that adding an NES sequence to a reporter protein that localizes to the nucleus shifted the localization to the cytoplasm (Stade et al., 1997, Fu et al., 2018). Similarly, attaching the $\mathrm{NES}_{\mathrm{Nup} 214}$ or $\mathrm{NES}_{\mathrm{HIV}-1 \text { Rev }}$ to the C-terminus of Nup214 having a mutation in the NES region, completely shifted the localization of the protein to the cytoplasm. The addition of LMB resulted in a nuclear localization of the protein, indicating that the artificial NESs could form a CRM1 dependent export complex.

In some previous studies, a series of NES peptides with variable affinities for CRM1 were identified (Güttler et al, 2010, Güttler et al, 2011). Overexpression in cells showed that NES peptides with low affinities for CRM1 had a mixed nuclear/cytoplasmic localization, while NES peptides with above average affinities for CRM1 such as YFP-NES $_{\mathrm{PKI}}$ overexpressed mainly in the cytoplasm (Fu et al., 2018). Similarly, GFP-NES $\mathrm{Nup}_{14}$ and GFP-NES $\mathrm{HIV}_{-1}$ Rev overexpressed in cells localized at the cytoplasm. The LMB results confirmed that both NESs are exported by CRM1. GFP-NES $\mathrm{Nup} 214_{14}$ in our study localized to the cytoplasm in a similar manner as the YFP-NES PKI $_{\text {shown by Fu et al. (2018). However, unlike YFP-NES }}$ PKI (shown by $\mathrm{Fu}$ et al., 2018) and HIV-1 Rev NES peptide shown in our study, NES Nup214 additionally localized at the NE. The findings confirm our immobilization results (Figure 12 \& Figure 13) 


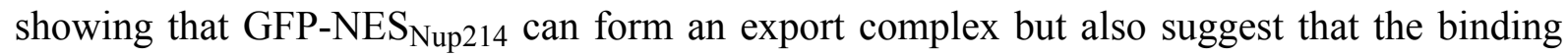
affinity of Nup214 NES to CRM1 is higher than the HIV-1 Rev NES. Furthermore, attaching an NES to the nuclear-localized overexpressed FG-repeat fragment translocated it to the cytoplasm and at the NE.

In conclusion, the high affinity of Nup214 NES for CRM1 is responsible for the localization of Nup214 to the cytoplasm and the NE. It is possible that this high affinity for CRM1 is required to keep the Nup214 for longer time at the NE, and hence Nup214 can be incorporated into the NPC.

\subsection{The Nup214-NES may play a role in targeting Nup214/Nup88 complex in a Nup358 dependent manner}

A GFP-NES $\mathrm{Nup}_{214}$ showed a prominent NE localization and cytoplasmic localization when overexpressed in cells. Additionally, CRM1 accumulates with $\mathrm{NES}_{\mathrm{Nup} 214}$ at the NE (Figure

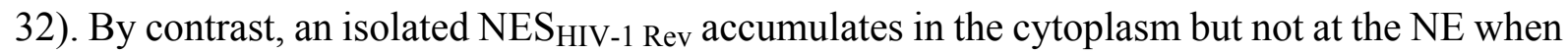
overexpressed in cells. In one study (Engelsma et al., 2004), the overexpression of a shuttling reporter protein containing GFP and an NES with very high affinity for CRM1, showed a prominent NE staining. In addition, the depletion of Nup358 resulted in loss of the NE staining (Engelsma et al., 2004). Similarly, knockdown experiments showed that the accumulation of GFP-Nup214 (L878A/L882A) with a NES $_{\text {Nup214 }}$ at the C-terminus at the NE was Nup358 dependent. Upon depletion of Nup358, the GFP-Nup214 (L878A/L882A) with a Nup214 NES at the C-terminus was localized to the cytoplasm only.

Proximity ligation assays (PLA) showed an LMB-insensitive interaction between Nup358 and CRM1 at the NE. Additionally, LMB had no effect on CRM1-Nup358 interaction in PLA experiments fitting with other reports where LMB did not disassemble CRM1 from its Nup358 binding sites (Singh et al, 1999).

The presence of an NES in Nup214 raises an important question about its contribution to the assembly of the Nup88-Nup214 at the cytoplasmic face of the NPC. The biochemical data revealed that Nup214 NES has a higher affinity for CRM1 than other NESs and that the complex formed with CRM1 was RanGTP dependent. This might result in the formation of an export complex that docks at Nup358 in the cytoplasm and hence get the Nup88-Nup214 subcomplex near to their incorporation site at the NPC. This might explain why GFP-Nup214 accumulates at the NPC. 
These results suggest that Nup358 is the docking site for CRM1 export complexes where they are disassembled. CRM1 export complexes that dock at Nup358 can be destabilized by addition of RanBP1 or RanGAP (Kehlenbach et al., 1999). However, digitonin-permeabilization experiments revealed that GFP-Nup214 accumulating at the NE was insensitive to RanBP1 and RanGAP. We concluded that this population got incorporated into the NE and hence cannot be released. A possible incorporation mechanism is that Nup88-Nup214 form a complex in the cytoplasm, which later interacts with free CRM1 and RanGTP available at the NPC and hence the Nup88-Nup214 complex gets incorporated into the NPC. Previous studies showed that depletion of Nup358 resulted in a slight reduction of Rev-NES nuclear export due to reduced recycling of CRM1 to the nucleus (Bernad, 2004). However, depletion of Nup358 in this study did not result in accumulation of overexpressed Nup214 in the nucleus but rather reduced its accumulation at the NE. By contrast, Hutten et al. (2006), showed that Nup358 depletion did not affect endogenous Nup214 presence at the NPC, suggesting that Nup214 may have different targeting pathways to the NPC. A possible explanation for our findings is that the high affinity of Nup214 NES to CRM1 kept Nup214 in the cytoplasm, but due to the absence of Nup358, Nup214 was not incorporated into the NPC. In fact, the role of karyopherins in targeting and assembly of nucleoporins has been previously reported. Lusk et.al. (2002), described the role of karyopherin Kap121p in the targeting and assembly of Nup53p in yeast NPC via a Nup53p NLS sequence. The study showed that the Kap121p-Nup53p complex was disassembled by binding to its binding partner Nup170p. Furthermore, an NLS sequence in Nup153 was characterized for its role in nuclear import of a shuttling population of Nup153 and assembling Nup153 at the nucleoplasm face of the NPC (Nakielny et al., 1999, Daigle et al., 2001). The presence of NLSs in Nic96p sequence (Grandi et al., 1995) and Nup153 (Nakielny et al., 1999) was speculated to play a role in importin-mediated integration of this nucleoporin into the NPC. According to Yavuz et al. (2010), an NLS sequence in Xenopus Pom121 was necessary for Pom121 interaction with Nup205, Nup160, Nup133 and Nup62. Additionally, the NLS of Pom121 is important for targeting of Pom121 to the NE, since an NLS truncated Pom121 localized to the cytoplasm rather than the NE. Furthermore, nuclear import was slower in cells expressing an NLS mutant version of Pom121 compared to cells expressing wild-type Pom121. An additional role of karyopherins in assembly of the NPC was described by Lu et al. (2012), where importin $\alpha / \beta$ recruit NLS containing proteins to chromatin at the end of mitosis to initiate NE assembly in Xenopus egg extracts. 


\subsection{A model for NES-dependent targeting of Nup214 to the NPC}

The results obtained in this thesis together with previously published reports can help generating models of Nup214 NES function. In one model, the Nup88-Nup214 subcomplex is formed in the cytoplasm and the Nup214 NES functions as a targeting signal that initiates a high affinity interaction with free CRM1 and RanGTP available at the Nup358. This interaction can bring the Nup88-Nup214 subcomplex in proximity with the nucleoporins forming the cytoplasmic face of the NPC (Figure 28a).

Another model suggests that Nup88-Nup214 subcomplex is formed in the cytoplasm and translocate to the nucleus by the help of Nup214 FG-repeats, but then quickly gets exported out of the nucleus through the formation of a CRM1/RanGTP dependent export complex. Once the Nup214 export complex is out of the nucleus, it docks at Nup358. At Nup358, the subcomplex gets in close proximity to the cytoplasmic face of the NPC and thus can get incorporated (Figure $28 b)$.

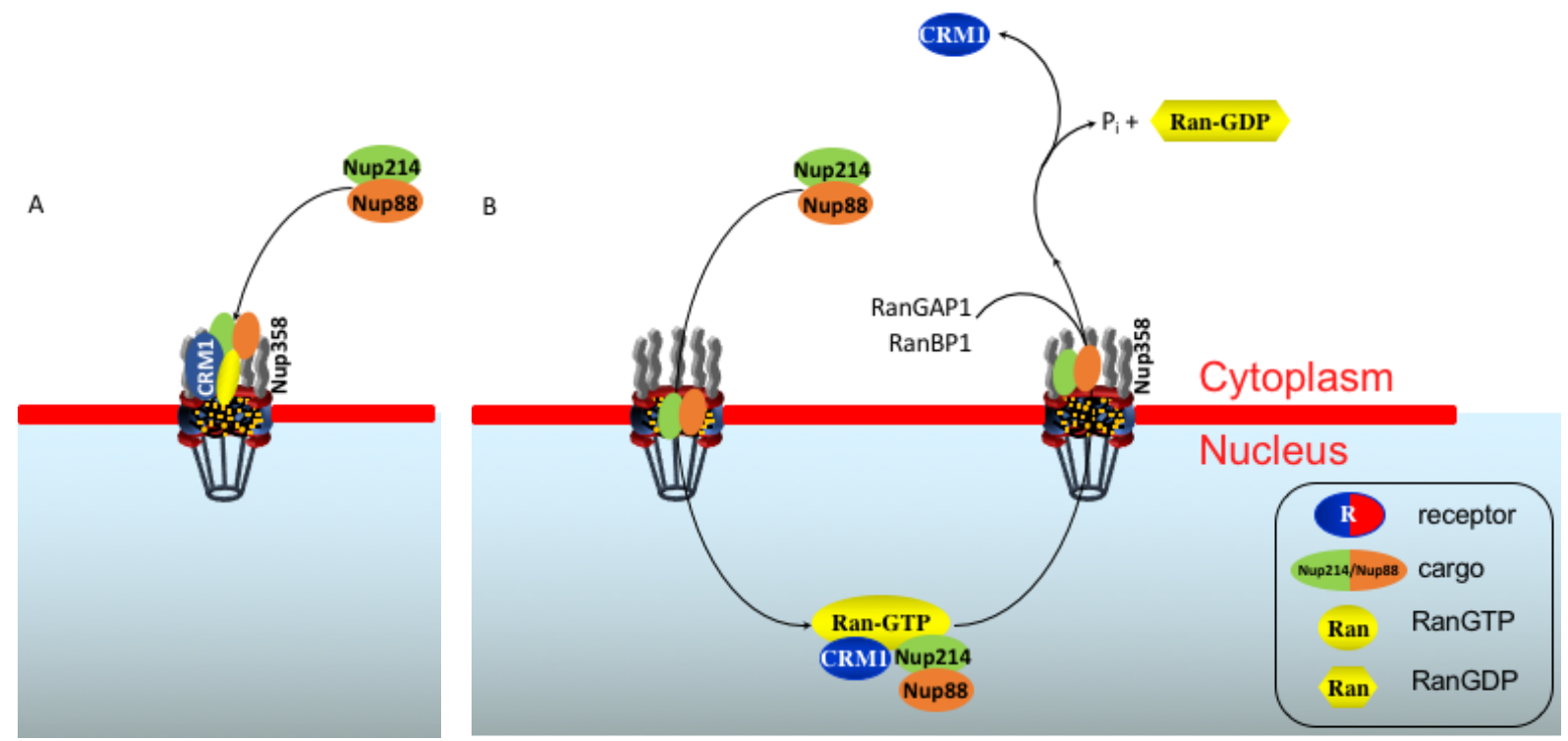

Figure 28: Models of Nup214 NES function. (A) Nup88-Nup214 subcomplex is formed in the cytoplasm and interacts with free CRM1 and RanGTP residing at Nup358. This leads to the accumulation of Nup88-Nup214 subcomplex at the NPC and hence get Nup88-Nup214 closer to their incorporation site at the NPC (B) Nup88-Nup214 subcomplex is formed in the cytoplasm then translocate to the nucleus by interaction of Nup214 FG-repeats with NPC central channel barrier. Once in the nucleus, an export complex of CRM1-RanGTP-Nup214-Nup88 is formed and translocates back to the cytoplasm, where it docks on Nup358. The export complex is dissociated by RanGAP1 and RanBP1, CRM1 is recycled and the Nup88-Nup214 subcomplex incorporates into the NPC. 


\section{Summary}

The NPC is a tightly regulated gateway that regulates the translocation of proteins in and out of the nucleus. The building unit of the NPC is a group of 30 distinctive proteins known as nucleoporins. The cytoplasmic face of the NPC consists of Nup62, Nup88, Nup214 and Nup358. Co-immunoprecipitation experiments showed that Nup88 and Nup214 exist as a subcomplex, where the two protein interact via their coiled-coil domain. However, the correlation between the three protein and their assembly pattern is still under investigation.

A putative NES sequence was identified in Nup214. This study aimed at characterizing this NES sequence with respect to a possible function in targeting and incorporation of Nup214 at the NPC. Overexpression experiments showed that exogenous Nup214 localizes at the cytoplasm and the NE. However, the addition of the prominent CRM1 inhibitor, LMB, shifted the localization of overexpressed Nup214 to the nucleus. The results showed that Nup214 can behave as a CRM1 export complex. Furthermore, a mutation in the NES sequence produced an effect similar to that of LMB.

Immobilization experiments confirmed that purified coiled-coil domain fragments of Nup214 interacted with CRM1 in a RanGTP dependent manner. In fact, a mutation in the NES sequence of Nup214 ceased the formation of the export complex. The affinity of Nup214 NES was measured by fluorescence polarization in comparison to established NESs as PKI and HIV-1 Rev. The results revealed a high affinity of Nup214 for CRM1. This suggests that Nup214 can disassemble some export complex by competing with their NESs

Additionally, this study investigated the effect of the $\mathrm{NES}_{\mathrm{Nup} 214}$ on the Nup88-Nup214 interaction. Overexpression of Nup214 sequestered endogenous and overexpressed Nup88 to cytoplasmic puncta. Additionally, LMB or a mutation in the NES sequence of Nup214 shifted the localization of both Nup214 and Nup88 to the nucleus. Co-immunoprecipitation experiments showed that a mutation in the NES region of Nup214 did not have an effect on the binding to Nup88. This indicates that Nup88-Nup214 subcomplex localization can change in an NES dependent manner. The depletion of Nup214 resulted in co-depletion of Nup88. However, re-expression of exogenous wild type Nup214 in Nup214-depleted cells, could rescue endogenous Nup88.

The role of the $\mathrm{NES}_{\mathrm{Nup} 214}$ in targeting of Nup214 to the NPC was investigated. RNAi experiments showed that a depletion of Nup358 resulted in loss of Nup214 at the NE. This study suggests a model of Nup88-Nup214 incorporation in the NE via a newly recognized NES sequence in Nup214 and in a CRM1- and possibly RanGTP-dependent manner. 


\section{Outlook}

The presence of an NES in Nup214 might indicate a role of Nup214 in export via competing with CRM1-mediated export complexes which depend on Nup214 for export such as the S60 ribosomal subunit. Further experiments are required to indicate the localization of Nup214dependent export complex. Detection for S60 ribosomal subunit in cell lines overexpressing Nup214 with a mutation in the NES sequence might reveal a shift in localization.

Another possible role of the NES of Nup214 might be linked to cell cycle. In this context, further analysis of the role of Nup358 and Nup88 in the assembly of Nup214 at the NPC has to be investigated. In addition, the role of the NES of Nup214 in the assembly of Nup214 has to be investigated closely. 


\section{References}

Adachi, Y., \& Yanagida, M. (1989). Higher order chromosome structure is affected by coldsensitive mutations in a Schizosaccharomyces pombe gene crm1+ which encodes a 115$\mathrm{kD}$ protein preferentially localized in the nucleus and its periphery. Journal of Cell Biology, 108(4), 1195-1207. https://doi.org/10.1083/jcb.108.4.1195

Adam, S. A., Marr, R. S., \& Gerace, L. (1990). Nuclear protein import in permeabilized mammalian cells requires soluble cytoplasmic factors. Journal of Cell Biology, 111(3), 807-816. https://doi.org/10.1083/jcb.111.3.807

Ader, C., Frey, S., Maas, W., Schmidt, H. B., Görlich, D., \& Baldus, M. (2010). Amyloid-like interactions within nucleoporin FG hydrogels. Proceedings of the National Academy of Sciences of the United States of America, 107(14), 6281-6285.

https://doi.org/10.1073/pnas.0910163107

Allen, N. P. C., Patel, S. S., Huang, L., Chalkley, R. J., Burlingame, A., Lutzmann, M., Hurt, E. C., \& Rexach, M. (2002). Deciphering networks of protein interactions at the nuclear pore complex. Molecular \& Cellular Proteomics, 1(12), 930-946. https://doi.org/10.1074/mcp.T200012-MCP200

Amlacher, S., Sarges, P., Flemming, D., Van Noort, V., Kunze, R., Devos, D. P., Arumugam, M., Bork, P., \& Hurt, E. (2011). Insight into structure and assembly of the nuclear pore complex by utilizing the genome of a eukaryotic thermophile. Cell, 146(2), 277-289. https://doi.org/10.1016/j.cell.2011.06.039

Antonin, W., Ellenberg, J., \& Dultz, E. (2008). Nuclear pore complex assembly through the cell cycle: Regulation and membrane organization. FEBS Letters, 582(14), 2004-2016. https://doi.org/10.1016/j.febslet.2008.02.067

Arlucea, J., Andrade, R., Alonso, R., \& Aréchaga, J. (1998). The nuclear basket of the nuclear pore complex is part of a higher- order filamentous network that is related to chromatin. Journal of Structural Biology, 124(1), 51-58. https://doi.org/10.1006/jsbi.1998.4054

Arnaoutov, A., Azuma, Y., Ribbeck, K., Joseph, J., Boyarchuk, Y., Karpova, T., McNally, J., \& Dasso, M. (2005). Crm1 is a mitotic effector of Ran-GTP in somatic cells. Nature Cell Biology, 7(6), 626-632. https://doi.org/10.1038/ncb1263

Arts, G. J., Fornerod, M., \& Mattaj, I. W. (1998). Identification of a nuclear export receptor for tRNA. Current Biology, 8(6), 305-314. https://doi.org/10.1016/S09609822(98)70130-7

Askjaer, P., Jensen, T. H., Nilsson, J., Englmeier, L., \& Kjems, J. (1998). The specificity of 
the CRM1-Rev nuclear export signal interaction is mediated by RanGTP. Journal of Biological Chemistry, 273(50), 33414-33422. https://doi.org/10.1074/jbc.273.50.33414

Bastos, R., De Pouplana, L. R., Enarson, M., Bodoor, K., \& Burke, B. (1997). Nup84, a novel nucleoporin that is associated with CAN/Nup214 on the cytoplasmic face of the nuclear pore complex. Journal of Cell Biology, 137(5), 989-1000. https://doi.org/10.1083/jcb.137.5.989

Bayliss, R., Corbett, A. H., \& Stewart, M. (2000). The molecular mechanism of transport of macromolecules through nuclear pore complexes. Traffic, 1(6), 448-456. https://doi.org/10.1034/j.1600-0854.2000.010602.x

Bayliss, R., Leung, S. W., Baker, R. P., Quimby, B. B., Corbett, A. H., \& Stewart, M. (2002). Structural basis for the interaction between NTF2 and nucleoporin FxFG repeats. EMBO Journal, 21(12), 2843-2853. https://doi.org/10.1093/emboj/cdf305

Bayliss, R., Ribbeck, K., Akin, D., Kent, H. M., Feldherr, C. M., Görlich, D., \& Stewart, M. (1999). Interaction between NTFP and $\mathrm{xFxFG-containing} \mathrm{nucleoporins} \mathrm{is} \mathrm{required} \mathrm{to}$ mediate nuclear import of RanGDP. Journal of Molecular Biology, 293(3), 579-593. https://doi.org/10.1006/jmbi.1999.3166

Beck, M., \& Baumeister, W. (2016). Cryo-Electron Tomography: Can it Reveal the Molecular Sociology of Cells in Atomic Detail? Trends in Cell Biology, 26(11), 825837. https://doi.org/10.1016/j.tcb.2016.08.006

Belgareh, N., Snay-Hodge, C., Pasteau, F., Dagher, S., Cole, C. N., \& Doye, V. (1998). Functional characterization of a Nup159p-containing nuclear pore subcomplex. Molecular Biology of the Cell, 9(12), 3475-3492. https://doi.org/10.1091/mbc.9.12.3475 Bernad, R., van der Velde, H., Fornerod, M., \& Pickersgill, H. (2004). Nup358/RanBP2 Attaches to the Nuclear Pore Complex via Association with Nup88 and Nup214/CAN and Plays a Supporting Role in CRM1-Mediated Nuclear Protein Export. Molecular and Cellular Biology, 24(6), 2373-2384. https://doi.org/10.1128/mcb.24.6.2373-2384.2004 Bernad, Rafael, Engelsma, D., Sanderson, H., Pickersgill, H., \& Fornerod, M. (2006). Nup214-Nup88 nucleoporin subcomplex is required for CRM1-mediated $60 \mathrm{~S}$ preribosomal nuclear export. Journal of Biological Chemistry, 281(28), 19378-19386. https://doi.org/10.1074/jbc.M512585200

Bischoff, F. R., Krebber, H., Smirnova, E., Dong, W., \& Ponstingl, H. (1995). Co-activation of RanGTPase and inhibition of GTP dissociation by Ran-GTP binding protein RanBP1. The EMBO Journal, 14(4), 705-715. https://doi.org/10.1002/j.14602075.1995.tb07049.x 
Bonner, W. M. (1975). Protein migration into nuclei: I. frog oocyte nuclei in vivo accumulate microinjected histones, allow entry to small proteins, and exclude large proteins. Journal of Cell Biology, 64(2), 421-430. https://doi.org/10.1083/jcb.64.2.421

Brohawn, S. G., Leksa, N. C., Spear, E. D., Rajashankar, K. R., \& Schwartz, T. U. (2008). Structural evidence for common ancestry of the nuclear pore complex and vesicle coats. Science, 322(5906), 1369-1373. https://doi.org/10.1126/science.1165886

Bui, K. H., Von Appen, A., Diguilio, A. L., Ori, A., Sparks, L., Mackmull, M. T., Bock, T., Hagen, W., Andrés-Pons, A., Glavy, J. S., \& Beck, M. (2013). Integrated structural analysis of the human nuclear pore complex scaffold. Cell, 155(6), 1233-1243. https://doi.org/10.1016/j.cell.2013.10.055

Cambridge, S. B., Gnad, F., Nguyen, C., Bermejo, J. L., Krüger, M., \& Mann, M. (2011). Systems-wide proteomic analysis in mammalian cells reveals conserved, functional protein turnover. Journal of Proteome Research, 10(12), 5275-5284. https://doi.org/10.1021/pr101183k

Charneau, P., Mirambeau, G., Roux, P., Paulous, S., Buc, H., \& Clavel, F. (1994). HIV-1 reverse transcription. A termination step at the center of the genome. Journal of Molecular Biology (Vol. 241, Issue 5, pp. 651-662). https://doi.org/10.1006/jmbi.1994.1542

Chen, Y. (2011). Calcium Phosphate Transfection of Eukaryotic Cells. Bio-Protocol, 1(7), 57.

Chook, Y. M., \& Blobel, G. (1999). Structure of the nuclear transport complex karyopherin32-Ran·GppNHp. Nature, 399(6733), 230-237. https://doi.org/10.1038/20375

Cook, A., Bono, F., Jinek, M., \& Conti, E. (2007). Structural Biology of Nucleocytoplasmic Transport. Annual Review of Biochemistry, 76(1), 647-671. https://doi.org/10.1146/annurev.biochem.76.052705.161529

Cordes, V. C., Reidenbach, S., Kohler, A., Stuurman, N., Van Driel, R., \& Franke, W. W. (1993). Intranuclear filaments containing a nuclear pore complex protein. Journal of Cell Biology, 123(6 I), 1333-1344. https://doi.org/10.1083/jcb.123.6.1333

Cronshaw, J. M., Krutchinsky, A. N., Zhang, W., Chait, B. T., \& Matunis, M. L. J. (2002). Proteomic analysis of the mammalian nuclear pore complex. Journal of Cell Biology, 158(5), 915-927. https://doi.org/10.1083/jcb.200206106

D’Angelo, M. A., Anderson, D. J., Richard, E., \& Hetzer, M. W. (2006). Nuclear pores form de novo from both sides of the nuclear envelope. Science, 312(5772), 440-443. https://doi.org/10.1126/science. 1124196 
De Souza, C. P. C., \& Osmani, S. A. (2007). Mitosis, not just open or closed. Eukaryotic Cell, 6(9), 1521-1527. https://doi.org/10.1128/EC.00178-07

Delphin, C., Guan, T., Melchior, F., \& Gerace, L. (1997). RanGTP targets p97 to RanBP2, a filamentous protein localized at the cytoplasmic periphery of the nuclear pore complex. Molecular Biology of the Cell, 8(12), 2379-2390. https://doi.org/10.1091/mbc.8.12.2379

Denning, D. P., Patel, S. S., Uversky, V., Fink, A. L., \& Rexach, M. (2003). Disorder in the nuclear pore complex: The FG repeat regions of nucleoporins are natively unfolded. Proceedings of the National Academy of Sciences of the United States of America, 100(5), 2450-2455. https://doi.org/10.1073/pnas.0437902100

Devos, D., Dokudovskaya, S., Alber, F., Williams, R., Chait, B. T., Sali, A., \& Rout, M. P. (2004). Components of coated vesicles and nuclear pore complexes share a common molecular architecture. PLoS Biology, 2(12). https://doi.org/10.1371/journal.pbio.0020380

Devos, D., Dokudovskaya, S., Williams, R., Alber, F., Eswar, N., Chait, B. T., Rout, M. P., \& Sali, A. (2006). Simple fold composition and modular architecture of the nuclear pore complex. Proceedings of the National Academy of Sciences of the United States of America, 103(7), 2172-2177. https://doi.org/10.1073/pnas.0506345103

Dirk, G., \& Kutay, U. (1999). Transport between the cell nucleus and the cytoplasm. Annu. Rev. Cell Dev. Biol., 15(607-60).

Dormann, D. (2020). FG-nucleoporins caught in the act of liquid-liquid phase separation. Journal of Cell Biology, 219(1), 10-11. https://doi.org/10.1083/jcb.201910211

DuBridge, R. B., Tang, P., Hsia, H. C., Leong, P. M., Miller, J. H., \& Calos, M. P. (1987). Analysis of mutation in human cells by using an Epstein-Barr virus shuttle system. Molecular and Cellular Biology, 7(1), 379-387. https://doi.org/10.1128/mcb.7.1.379

Dultz, E., \& Ellenberg, J. (2010). Live imaging of single nuclear pores reveals unique assembly kinetics and mechanism in interphase. Journal of Cell Biology, 191(1), 15-22. https://doi.org/10.1083/jcb.201007076

Dultz, E., Zanin, E., Wurzenberger, C., Braun, M., Rabut, G., Sironi, L., \& Ellenberg, J. (2008). Systematic kinetic analysis of mitotic dis- and reassembly of the nuclear pore in living cells. Journal of Cell Biology, 180(5), 857-865. https://doi.org/10.1083/jcb.200707026

Eichwald, V., Daeffler, L., Klein, M., Rommelaere, J., \& Salome, N. (2002). The NS2 Proteins of Parvovirus Minute Virus of Mice Are Required for Efficient Nuclear Egress of Progeny Virions in Mouse Cells. Journal of Virology, 76(20), 10307-10319. 
https://doi.org/10.1128/jvi.76.20.10307-10319.2002

Eisenhardt, N., Redolfi, J., \& Antonin, W. (2014). Interaction of Nup53 with Ndc1 and Nup155 is required for nuclear pore complex assembly. Journal of Cell Science, 127(4), 908-921. https://doi.org/10.1242/jcs.141739

Engelsma, D., Bernad, R., Calafat, J., \& Fornerod, M. (2004). Supraphysiological nuclear export signals bind CRM1 independently of RanGTP and arrest at Nup358. EMBO Journal, 23(18), 3643-3652. https://doi.org/10.1038/sj.emboj.7600370

Etchin, J., Sun, Q., Kentsis, A., Farmer, A., Zhang, Z. C., Sanda, T., Mansour, M. R., Barcelo, C., McCauley, D., Kauffman, M., Shacham, S., Christie, A. L., Kung, A. L., Rodig, S. J., Chook, Y. M., \& Look, A. T. (2013). Antileukemic activity of nuclear export inhibitors that spare normal hematopoietic cells. Leukemia, 27(1), 66-74.

https://doi.org/10.1038/leu.2012.219

Fabre, E., \& Hurt, E. (1997). Yeast Genetics To Dissect the Nuclear Pore Complex and Nucleocytoplasmic Trafficking. Annual Review of Genetics, 31(1), 277-313. https://doi.org/10.1146/annurev.genet.31.1.277

Fischer, J., Teimer, R., Amlacher, S., Kunze, R., \& Hurt, E. (2015). Linker Nups connect the nuclear pore complex inner ring with the outer ring and transport channel. Nature Structural and Molecular Biology, 22(10), 774-781. https://doi.org/10.1038/nsmb.3084

Fischer, U., Huber, J., Boelens, W. C., Mattajt, L. W., \& Lührmann, R. (1995). The HIV-1 Rev Activation Domain is a nuclear export signal that accesses an export pathway used by specific cellular RNAs. Cell, 82(3), 475-483. https://doi.org/10.1016/00928674(95)90436-0

Flemming, D., Sarges, P., Stelter, P., Hellwig, A., Bottcher, B., \& Hurt, E. (2009). Two structurally distinct domains of the nucleoporin Nup170 cooperate to tether a subset of nucleoporins to nuclear pores. Journal of Cell Biology, 185(3), 387-395. https://doi.org/10.1083/jcb.200810016

Fornerod, M., Ohno, M., Yoshida, M., \& Mattaj, I. W. (1997a). CRM1 is an export receptor for leucine-rich nuclear export signals. Cell, 90(6), 1051-1060. https://doi.org/10.1016/S0092-8674(00)80371-2

Fornerod, M., Van Deursen, J., Van Baal, S., Reynolds, A., Davis, D., Murti, K. G., Fransen, J., \& Grosveld, G. (1997b). The human homologue of yeast CRM1 is in a dynamic subcomplex with CAN/Nup214 and a novel nuclear pore component Nup88. EMBO Journal, 16(4), 807-816. https://doi.org/10.1093/emboj/16.4.807

Franz, C., Walczak, R., Yavuz, S., Santarella, R., Gentzel, M., Askjaer, P., Galy, V., Hetzer, 
M., Mattaj, I. W., \& Antonin, W. (2007). MEL-28/ELYS is required for the recruitment of nucleoporins to chromatin and postmitotic nuclear pore complex assembly. EMBO Reports, 8(2), 165-172. https://doi.org/10.1038/sj.embor.7400889

Frey, S., Richter, R. P., \& Görlich, D. (2006). FG-rich repeats of nuclear pore proteins form a three-dimensional meshwork with hydrogel-like properties. Science, 314(5800), 815817. https://doi.org/10.1126/science. 1132516

Fried, H., \& Kutay, U. (2003). Nucleocytoplasmic transport: Taking an inventory. Cellular and Molecular Life Sciences, 60(8), 1659-1688. https://doi.org/10.1007/s00018-0033070-3

Frohnert, C., Hutten, S., Wälde, S., Nath, A., \& Kehlenbach, R. H. (2014). Importin 7 and Nup358 promote nuclear import of the protein component of human telomerase. PLoS ONE, 9(2). https://doi.org/10.1371/journal.pone.0088887

Fu, S. C., Fung, H. Y. J., Cağatay, T., Baumhardt, J., \& Chook, Y. M. (2018). Correlation of CRM1-NES affinity with nuclear export activity. Molecular Biology of the Cell, 29(17), 2037-2044. https://doi.org/10.1091/mbc.E18-02-0096

Fu, S. C., Huang, H. C., Horton, P., \& Juan, H. F. (2013). ValidNESs: A database of validated leucine-rich nuclear export signals. Nucleic Acids Research, 41(D1), 338-343. https://doi.org/10.1093/nar/gks936

Funakoshi, T., Clever, M., Watanabe, A., \& Imamoto, N. (2011). Localization of Pom121 to the inner nuclear membrane is required for an early step of interphase nuclear pore complex assembly. Molecular Biology of the Cell, 22(7), 1058-1069.

https://doi.org/10.1091/mbc.E10-07-0641

Fung, H. Y. J., Fu, S. C., Brautigam, C. A., \& Chook, Y. M. (2015). Structural determinants of nuclear export signal orientation in binding to exportin CRM1. ELife, 4, 1-19. https://doi.org/10.7554/eLife.10034

Gaik, M., Flemming, D., Von Appen, A., Kastritis, P., Mücke, N., Fischer, J., Stelter, P., Ori, A., Bui, K. H., Baßler, J., Barbar, E., Beck, M., \& Hurt, E. (2015). Structural basis for assembly and function of the Nup82 complex in the nuclear pore scaffold. Journal of Cell Biology, 208(3), 283-297. https://doi.org/10.1083/jcb.201411003

Gilistro, E., de Turris, V., Damizia, M., Verrico, A., Moroni, S., De Santis, R., Rosa, A., \& Lavia, P. (2017). Importin- $\beta$ and CRM1 control a RANBP2 spatiotemporal switch essential for mitotic kinetochore function. Journal of Cell Science, 130(15), 2564-2578. https://doi.org/10.1242/jcs. 197905

Gluzman, Y. (1981). SV40-transformed simian cells support the replication of early SV40 
mutants. Cell, 23(1), 175-182. https://doi.org/10.1016/0092-8674(81)90282-8

Goldberg, M. W., \& Allen, T. D. (1992). High resolution scanning electron microscopy of the nuclear envelope: Demonstration of a new, regular, fibrous lattice attached to the baskets of the nucleoplasmic face of the nuclear pores. Journal of Cell Biology, 119(6), 14291440. https://doi.org/10.1083/jcb.119.6.1429

Goldfarb, D. S., Corbett, A. H., Mason, D. A., Harreman, M. T., \& Adam, S. A. (2004). Importin $\alpha$ : A multipurpose nuclear-transport receptor. Trends in Cell Biology, 14(9), 505-514. https://doi.org/10.1016/j.tcb.2004.07.016

Görlich, D., Panté, N., Kutay, U., Aebi, U., \& Bischoff, F. R. (1996). Identification of different roles for RanGDP and RanGTP in nuclear protein import. EMBO Journal, 15(20), 5584-5594. https://doi.org/10.1002/j.1460-2075.1996.tb00943.x

Görlich, D., Vogelt, F., Mills, A. D., Hartmannt, E., \& Laskey, R. A. (1995). in nuclear protein import. Letters to nature, 3, 246-248.

Görlich, Dirk, Dabrowski, M., Bischoff, F. R., Kutay, U., Bork, P., Hartmann, E., Prehn, S., \& Izaurralde, E. (1997). A novel class of RanGTP binding proteins. Journal of Cell Biology, 138(1), 65-80. https://doi.org/10.1083/jcb.138.1.65

Gorsch, L., Dockendorff, T.C., Cole, C. N. (1995). A Conditional Allele of the Novel Repeatcontaining Yeast Nucleoporin. Jcb, 129(4), 939-955.

Grandi, P., Doye, V., \& Hurt, E. C. (1993). Purification of NSP1 reveals complex formation with 'GLFG' nucleoporins and a novel nuclear pore protein NIC96. EMBO Journal, 12(8), 3061-3071. https://doi.org/10.1002/j.1460-2075.1993.tb05975.x

Grandi, P., Schlaich, N., Tekotte, H., \& Hurt, E. C. (1995). Functional interaction of Nic96p with a core nucleoporin complex consisting of Nsp1p, Nup49p and a novel protein Nup57p. EMBO Journal, 14(1), 76-87. https://doi.org/10.1002/j.14602075.1995.tb06977.x

Gullberg M, Gústafsdóttir S.M., Schallmeiner E., Jarvius J., Bjarnegård M., Betsholtz C., Landegren U., \& Fredriksson S. (2004). Cytokine detection by antibody-based proximity ligation. PNAS, 101 (22) 8420-8424; DOI: 10.1073/pnas.0400552101

Güttler, T., \& Görlich, D. (2011). Ran-dependent nuclear export mediators: A structural perspective. EMBO Journal, 30(17), 3457-3474.

https://doi.org/10.1038/emboj.2011.287

Güttler, T., Madl, T., Neumann, P., Deichsel, D., Corsini, L., Monecke, T., Ficner, R., Sattler, M., \& Görlich, D. (2010). NES consensus redefined by structures of PKI-type and Revtype nuclear export signals bound to CRM1. Nature Structural and Molecular Biology, 
17(11), 1367-1376. https://doi.org/10.1038/nsmb.1931

Hamamoto, T., Beppu, T., \& Seto, H. (1983). Leptomycins A and B, new antifungal antibiotics ii. structure elucidation. The Journal of Antibiotics, 36(6), 646-650. https://doi.org/10.7164/antibiotics.36.646

Hampoelz, B., Mackmull, M. T., Machado, P., Ronchi, P., Bui, K. H., Schieber, N., Santarella-Mellwig, R., Necakov, A., Andrés-Pons, A., Philippe, J. M., Lecuit, T., Schwab, Y., \& Beck, M. (2016). Pre-assembled Nuclear Pores Insert into the Nuclear Envelope during Early Development. Cell, 166(3), 664-678. https://doi.org/10.1016/j.cell.2016.06.015

Hampoelz, B., Schwarz, A., Ronchi, P., Bragulat-Teixidor, H., Tischer, C., Gaspar, I., Ephrussi, A., Schwab, Y., \& Beck, M. (2019). Nuclear Pores Assemble from Nucleoporin Condensates During Oogenesis. Cell, 179(3), 671-686.e17. https://doi.org/10.1016/j.cell.2019.09.022

Hashizume, C., Nakano, H., Yoshida, K., \& Wong, R. W. (2010). Characterization of the role of the tumor marker Nup88 in mitosis. Molecular Cancer, 9, 1-7. https://doi.org/10.1186/1476-4598-9-119

Hetzer, M. W., Walther, T. C., \& Mattaj, I. W. (2005). PUSHING THE ENVELOPE: Structure, Function, and Dynamics of the Nuclear Periphery. Annual Review of Cell and Developmental Biology, 21(1), 347-380. https://doi.org/10.1146/annurev.cellbio.21.090704.151152

Hopper, A. K., Traglia, H. M., \& Dunst, R. W. (1990). The yeast RNA1 gene product necessary for RNA processing is located in the cytosol and apparently excluded from the nucleus. Journal of Cell Biology, 111(2), 309-321. https://doi.org/10.1083/jcb.111.2.309

Hurwitz, M. E., Strambio-De-Castillia, C., \& Blobel, G. (1998). Two yeast nuclear pore complex proteins involved in mRNA export form a cytoplasmically oriented subcomplex. Proceedings of the National Academy of Sciences of the United States of America, 95(19), 11241-11245. https://doi.org/10.1073/pnas.95.19.11241

Hutten, S., \& Kehlenbach, R. H. (2006). Nup214 Is Required for CRM1-Dependent Nuclear Protein Export In Vivo. Molecular and Cellular Biology, 26(18), 6772-6785. https://doi.org/10.1128/mcb.00342-06

Iovine, M. K., Watkins, J. L., \& Wente, S. R. (1995). The GLFG repetitive region of the nucleoporin Nup116p interacts with Kap95p, an essential yeast nuclear import factor. Journal of Cell Biology, 131(6 II), 1699-1713. https://doi.org/10.1083/jcb.131.6.1699 Izaurralde, E., Kutay, U., Von Kobbe, C., Mattaj, L. W., \& Görlich, D. (1997). The 
asymmetric distribution of the constituents of the Ran system is essential for transport into and out of the nucleus. EMBO Journal, 16(21), 6535-6547.

https://doi.org/10.1093/emboj/16.21.6535

Jarnik, M., \& Aebi, U. (1991). Toward a more complete 3-D structure of the nuclear pore complex. Journal of Structural Biology, 107(3), 291-308. https://doi.org/10.1016/10478477(91)90054-Z

Joseph, J., Tan, S. H., Karpova, T. S., McNally, J. G., \& Dasso, M. (2002). SUMO-1 targets RanGAP1 to kinetochores and mitotic spindles. Journal of Cell Biology, 156(4), 595602. https://doi.org/10.1083/jcb.200110109

Kabachinski, G., \& Schwartz, T. U. (2015). The nuclear pore complex - Structure and function at a glance. Journal of Cell Science, 128(3), 423-429. https://doi.org/10.1242/jcs.083246

Kehlenbach, R. H., Dickmanns, A., \& Gerace, L. (1998). Nucleocytoplasmic shuttling factors including Ran and CRM1 mediate nuclear export of NFAT in vitro. Journal of Cell Biology, 141(4), 863-874. https://doi.org/10.1083/jcb.141.4.863

Kehlenbach, R. H., Dickmanns, A., Kehlenbach, A., Guan, T., \& Gerace, L. (1999). A role for RanBP1 in the release of CRM1 from the nuclear pore complex in a terminal step of nuclear export. Journal of Cell Biology, 145(4), 645-657.

Kerr, A. R. W., \& Schirmer, E. C. (2011). FG repeats facilitate integral protein trafficking to the inner nuclear membrane. Communicative \& Integrative Biology, 4(5), 557-559. https://doi.org/10.4161/cib.16052

Khalaf, B., Roncador, A., Pischedda, F., Casini, A., Thomas, S., Piccoli, G., Kiebler, M., \& Macchi, P. (2019). Ankyrin-G induces nucleoporin Nup358 to associate with the axon initial segment of neurons. Journal of Cell Science, 132(18), jcs222802. https://doi.org/10.1242/jcs.222802

Kırlı, K., Karaca, S., Dehne, H. J., Samwer, M., Pan, K. T., Lenz, C., Urlaub, H., \& Görlich, D. (2015). A deep proteomics perspective on CRM1-mediated nuclear export and nucleocytoplasmic partitioning. ELife, 4, 1-28. https://doi.org/10.7554/eLife.11466

Kosinski, J., Mosalaganti, S., Appen, A. Von, Teimer, R., Diguilio, A. L., Wan, W., Bui, K. H., Hagen, W. J. H., Briggs, J. a G., Glavy, J. S., Hurt, E., \& Beck, M. (2016). Pore Complex. 352(6283), 363-365.

Kraemer, D., Wozniak, R. W., Blobel, G., \& Radu, A. (1994). The human CAN protein, a putative oncogene product associated with myeloid leukemogenesis, is a nuclear pore complex protein that faces the cytoplasm. Proceedings of the National Academy of 
Sciences of the United States of America, 91(4), 1519-1523.

https://doi.org/10.1073/pnas.91.4.1519

Kudo, N., Wolff, B., Sekimoto, T., Schreiner, E. P., Yoneda, Y., Yanagida, M., Horinouchi, S., \& Yoshida, M. (1998). Leptomycin B inhibition of signal-mediated nuclear export by direct binding to CRM1. Experimental Cell Research, 242(2), 540-547. https://doi.org/10.1006/excr.1998.4136

Künzler, M., \& Hurt, E. (2001). Targeting of Ran: Variation on a common theme? Journal of Cell Science, 114(18), 3233-3241.

Kutay, U., \& Güttinger, S. (2005). Leucine-rich nuclear-export signals: Born to be weak. Trends in Cell Biology, 15(3), 121-124. https://doi.org/10.1016/j.tcb.2005.01.005

Laurell, E., Beck, K., Krupina, K., Theerthagiri, G., Bodenmiller, B., Horvath, P., Aebersold, R., Antonin, W., \& Kutay, U. (2011). Phosphorylation of Nup98 by multiple kinases is crucial for NPC disassembly during mitotic entry. Cell, 144(4), 539-550. https://doi.org/10.1016/j.cell.2011.01.012

Lee, Y., Pei, J., Baumhardt, J. M., Chook, Y. M., \& Grishin, N. V. (2019). Structural prerequisites for CRM1-dependent nuclear export signaling peptides: accessibility, adapting conformation, and the stability at the binding site. Scientific Reports, 9(1), 113. https://doi.org/10.1038/s41598-019-43004-0

Lim, R. Y. H., Huang, N. P., Köser, J., Deng, J., Aaron Lau, K. H., Schwarz-Herion, K., Fahrenkrog, B., \& Aebi, U. (2006). Flexible phenylalanine-glycine nucleoporins as entropic barriers to nucleocytoplasmic transport. Proceedings of the National Academy of Sciences of the United States of America, 103(25), 9512-9517. https://doi.org/10.1073/pnas.0603521103

Lin, D. H., \& Hoelz, A. (2019). The Structure of the Nuclear Pore Complex (An Update). Annual Review of Biochemistry, 88(1), 725-783. https://doi.org/10.1146/annurevbiochem-062917-011901

Lin, D. H., Stuwe, T., Schilbach, S., Rundlet, E. J., Perriches, T., Mobbs, G., Fan, Y., Thierbach, K., Huber, F. M., Collins, L. N., Davenport, A. M., Jeon, Y. E., \& Hoelz, A. (2016). Architecture of the symmetric core of the nuclear pore. Science, 352(6283). https://doi.org/10.1126/science.aaf1015

Lutzmann, M., Kunze, R., Buerer, A., Aebi, U., \& Hurt, E. (2002). Modular self-assembly of a Y-shaped multiprotein complex from seven nucleoporins. EMBO Journal, 21(3), 387397. https://doi.org/10.1093/emboj/21.3.387

Mahajan, R., Delphin, C., Guan, T., Gerace, L., \& Melchior, F. (1997). A small ubiquitin- 
related polypeptide involved in targeting RanGAP1 to nuclear pore complex protein RanBP2. Cell, 88(1), 97-107. https://doi.org/10.1016/S0092-8674(00)81862-0

Mahamid, J., Pfeffer, S., Schaffer, M., Villa, E., Danev, R., Cuellar, L. K., Förster, F., Hyman, A. A., Plitzko, J. M., \& Baumeister, W. (2016). Visualizing the molecular sociology at the HeLa cell nuclear periphery. Science, 351(6276), 969-972. https://doi.org/10.1126/science.aad8857

Maimon, T., Elad, N., Dahan, I., \& Medalia, O. (2012). The human nuclear pore complex as revealed by cryo-electron tomography. Structure, 20(6), 998-1006. https://doi.org/10.1016/j.str.2012.03.025

Makio, T., Stanton, L. H., Lin, C. C., Goldfarb, D. S., Weis, K., \& Wozniak, R. W. (2009). The nucleoporins Nup170p and Nup157p are essential for nuclear pore complex assembly. Journal of Cell Biology, 185(3), 459-473. https://doi.org/10.1083/jcb.200810029

Melchior, F., Guan, T., Yokoyama, N., Nishimoto, T., \& Gerace, L. (1995). GTP hydrolysis by Ran occurs at the nuclear pore complex in an early step of protein import. Journal of Cell Biology, 131(3), 571-581. https://doi.org/10.1083/jcb.131.3.571

Middleton, C. A., \& Sharp, J. A. (1984). Cytoplasmic Filaments. Cell Locomotion in Vitro, 56-83. https://doi.org/10.1007/978-1-4615-9772-8_4

Mingot, J. M., Kostka, S., Kraft, R., Hartmann, E., \& Görlich, D. (2001). Importin 13: A novel mediator of nuclear import and export. EMBO Journal, 20(14), 3685-3694. https://doi.org/10.1093/emboj/20.14.3685

Monecke, T., Güttler, T., Neumann, P., Dickmanns, A., Görlich, D., \& Ficner, R. (2009). Crystal structure of the nuclear export receptor CRM1 in complex with snurportin1 and RanGTP. Science, 324(5930), 1087-1091. https://doi.org/10.1126/science.1173388

Montpetit, B., Thomsen, N. D., Helmke, K. J., Seeliger, M. A., Berger, J. M., \& Weis, K. (2011). A conserved mechanism of DEAD-box ATPase activation by nucleoporins and InsP6 in mRNA export. Nature, 472(7342), 238-244. https://doi.org/10.1038/nature09862

Moroianu, J., Hijikata, M., Blobel, G., \& Radu, A. (1995). Mammalian karyopherin $\alpha 1 \beta$ and $\alpha 2 \beta$ heterodimers: $\alpha 1$ or $\alpha 2$ subunit binds nuclear localization signal and $\beta$ subunit interacts with peptide repeat-containing nucleoporins. Proceedings of the National Academy of Sciences of the United States of America, 92(14), 6532-6536. https://doi.org/10.1073/pnas.92.14.6532

Mosammaparast, N., \& Pemberton, L. F. (2004). Karyopherins: From nuclear-transport 
mediators to nuclear-function regulators. Trends in Cell Biology, 14(10), 547-556. https://doi.org/10.1016/j.tcb.2004.09.004

Muqbil, I., Azmi, A. S., \& Mohammad, R. M. (2018). Nuclear export inhibition for pancreatic cancer therapy. Cancers, 10(5), 1-14. https://doi.org/10.3390/cancers 10050138

Murawala, P., Tripathi, M. M., Vyas, P., Salunke, A., \& Joseph, J. (2009). Nup358 interacts with APC and plays a role in cell polarization. Journal of Cell Science, 122(17), 31133122. https://doi.org/10.1242/jcs.037523

Nachury, M. V., \& Weis, K. (1999). The direction of transport through the nuclear pore can be inverted. Proceedings of the National Academy of Sciences of the United States of America, 96(17), 9622-9627. https://doi.org/10.1073/pnas.96.17.9622

Napetschnig, J., Blobel, G., \& Hoelz, A. (2007). Crystal structure of the N-terminal domain of the human protooncogene Nup214/CAN. Proceedings of the National Academy of Sciences of the United States of America, 104(6), 1783-1788.

https://doi.org/10.1073/pnas.0610828104

Nemergut, M. E., Mizzen, C. A., Stukenberg, T., Allis, C. D., \& Macara, I. G. (2001). Chromatin docking and exchange activity enhancement of RCC1 by histones $\mathrm{H} 2 \mathrm{~A}$ and H2B. Science, 292(5521), 1540-1543. https://doi.org/10.1126/science.292.5521.1540

Neville, M., Stutz, F., Lee, L., Davis, L. I., \& Rosbash, M. (1997). The importin-beta family member Crm $1 \mathrm{p}$ bridges the interaction between Rev and the nuclear pore complex during nuclear export. Current Biology, 7(10), 767-775. https://doi.org/10.1016/S09609822(06)00335-6

Nishi, K., Yoshida, M., Fujiwara, D., Nishikawa, M., Horinouchi, S., \& Beppu, T. (1994). Leptomycin B targets a regulatory cascade of crm1, a fission yeast nuclear protein, involved in control of higher order chromosome structure and gene expression. Journal of Biological Chemistry, 269(9), 6320-6324.

Ocwieja, K. E., Brady, T. L., Ronen, K., Huegel, A., Roth, S. L., Schaller, T., James, L. C., Towers, G. J., Young, J. A. T., Chanda, S. K., König, R., Malani, N., Berry, C. C., \& Bushman, F. D. (2011). HIV integration targeting: A pathway involving transportin-3 and the nuclear pore protein RanBP2. PLoS Pathogens, 7(3), 19-21. https://doi.org/10.1371/journal.ppat.1001313

Ohtsubo, M., Okazaki, H., \& Nishimoto, T. (1989). The RCC1 protein, a regulator for the onset of chromosome condensation locates in the nucleus and binds to DNA. Journal of Cell Biology, 109(4 I), 1389-1397. https://doi.org/10.1083/jcb.109.4.1389

Onischenko, E. A., Gubanova, N. V., Kielselbach, T., Kiseleva, E. V., \& Hallberg, E. (2004). 
Annulate lamellae play only a minor role in the storage of excess nucleoporins in Drosophila embryos. Traffic, 5(3), 152-164. https://doi.org/10.1111/j.16000854.2004.0166.x

Onischenko, E., Stanton, L. H., Madrid, A. S., Kieselbach, T., \& Weis, K. (2009). Role of the $\mathrm{Ndcl}$ interaction network in yeast nuclear pore complex assembly and maintenance. Journal of Cell Biology, 185(3), 475-491. https://doi.org/10.1083/jcb.200810030

Ossareh-Nazari, B., Bachelerie, F., \& Dargemont, C. (1997). Evidence for a role of CRM1 in signal-mediated nuclear protein export. Science, 278(5335), 141-144. https://doi.org/10.1126/science.278.5335.141

Otsuka, S., Bui, K. H., Schorb, M., Julius Hossain, M., Politi, A. Z., Koch, B., Eltsov, M., Beck, M., \& Ellenberg, J. (2016). Nuclear pore assembly proceeds by an inside-out extrusion of the nuclear envelope. ELife, 5, 1-23. https://doi.org/10.7554/eLife.19071

Otsuka, S., \& Ellenberg, J. (2018). Mechanisms of nuclear pore complex assembly - two different ways of building one molecular machine. FEBS Letters, 592(4), 475-488. https://doi.org/10.1002/1873-3468.12905

Panté, N., \& Kann, M. (2002). Nuclear pore complex is able to transport macromolecules with diameters of $39 \mathrm{~nm}$. Molecular Biology of the Cell, 13(2), 425-434. https://doi.org/10.1091/mbc.01-06-0308

Pemberton, L. F., \& Paschal, B. M. (2005). Mechanisms of receptor-mediated nuclear import and nuclear export. Traffic, 6(3), 187-198. https://doi.org/10.1111/j.16000854.2005.00270.x

Peters, R. (2009). Translocation through the nuclear pore: Kaps pave the way. BioEssays, 31(4), 466-477. https://doi.org/10.1002/bies.200800159

Pichler, A., \& Melchior, F. (2002). Ubiquitin-related modifier SUMO1 and nucleocytoplasmic transport. Traffic, 3(6), 381-387. https://doi.org/10.1034/j.16000854.2002.30601.x

Port, S. A., Mendes, A., Valkova, C., Spillner, C., Fahrenkrog, B., Kaether, C., \& Kehlenbach, R. H. (2016). The oncogenic fusion proteins SET-Nup214 and sequestosome-1 (SQSTM1)-Nup214 form dynamic nuclear bodies and differentially affect nuclear protein and Poly(A)+ RNA export. Journal of Biological Chemistry, 291(44), 23068-23083. https://doi.org/10.1074/jbc.M116.735340

Port, S. A., Monecke, T., Dickmanns, A., Spillner, C., Hofele, R., Urlaub, H., Ficner, R., \& Kehlenbach, R. H. (2015). Structural and Functional Characterization of CRM1-Nup214 Interactions Reveals Multiple FG-Binding Sites Involved in Nuclear Export. Cell 
Reports, 13(4), 690-702. https://doi.org/10.1016/j.celrep.2015.09.042

Pouton, C. W., Wagstaff, K. M., Roth, D. M., Moseley, G. W., \& Jans, D. A. (2007).

Targeted delivery to the nucleus. Advanced Drug Delivery Reviews, 59(8), 698-717. https://doi.org/10.1016/j.addr.2007.06.010

Reichelt, R., Holzenburg, A., Buhle, E. L., Jarnik, M., Engel, A., \& Aebi, U. (1990). Correlation between structure and mass distribution of the nuclear pore complex and of distinct pore complex components. Journal of Cell Biology, 110(4), 883-894. https://doi.org/10.1083/jcb.110.4.883

Ribbeck, K. \& Görlich, D. (1998). NTF2 mediates nuclear import of Ran. The EMBO Journal, 17(22), 6587-6598. https://doi.org/10.1093/emboj/17.22.6587

Ribbeck, K., \& Görlich, D. (2001). Kinetic analysis of translocation through nuclear pore complexes. EMBO Journal, 20(6), 1320-1330. https://doi.org/10.1093/emboj/20.6.1320

Richards, S. A., Lounsbury, K. M., Carey, K. L., \& Macara, I. G. (1996). A nuclear export signal is essential for the cytosolic localization of the Ran binding protein, RanBP1. Journal of Cell Biology, 134(5), 1157-1168. https://doi.org/10.1083/jcb.134.5.1157

Richardson, W. D., Mills, A. D., Dilworth, S. M., Laskey, R. A., \& Dingwall, C. (1988). Nuclear protein migration involves two steps: Rapid binding at the nuclear envelope followed by slower translocation through nuclear pores. Cell, 52(5), 655-664. https://doi.org/10.1016/0092-8674(88)90403-5

Ris, H. (1997). High-resolution field-emission scanning electron microscopy of nuclear pore complex. Scanning, 19(5), 368-375. https://doi.org/10.1002/sca.4950190504

Ritterhoff, T., Das, H., Hofhaus, G., Schröder, R. R., Flotho, A., \& Melchior, F. (2016). The RanBP2/RanGAP1*SUMO1/Ubc9 SUMO E3 ligase is a disassembly machine for Crm1-dependent nuclear export complexes. Nature Communications, 7(May). https://doi.org/10.1038/ncomms11482

Roloff, S., Spillner, C., \& Kehlenbach, R. H. (2013). Several phenylalanine-glycine motives in the nucleoporin Nup214 are essential for binding of the nuclear export receptor CRM1. Journal of Biological Chemistry, 288(6), 3952-3963. https://doi.org/10.1074/jbc.M112.433243

Rothballer, A., \& Kutay, U. (2013). Poring over pores: Nuclear pore complex insertion into the nuclear envelope. Trends in Biochemical Sciences, 38(6), 292-301. https://doi.org/10.1016/j.tibs.2013.04.001

Rout, M. P., Aitchison, J. D., Magnasco, M. O., \& Chait, B. T. (2003). Virtual gating and nuclear transport: The hole picture. Trends in Cell Biology, 13(12), 622-628. 
https://doi.org/10.1016/j.tcb.2003.10.007

Rout, M. P., Aitchison, J. D., Suprapto, A., Hjertaas, K., Zhao, Y., \& Chait, B. T. (2000). The yeast nuclear pore complex: Composition, architecture, transport mechanism. Journal of Cell Biology, 148(4), 635-651. https://doi.org/10.1083/jcb.148.4.635

Ryan, K. J., McCaffery, J. M., \& Wente, S. R. (2003). The Ran GTPase cycle is required for yeast nuclear pore complex assembly. Journal of Cell Biology, 160(7), 1041-1053. https://doi.org/10.1083/jcb.200209116

Ryan, K. J., \& Wente, S. R. (2002). Isolation and characterization of new Saccharomyces cerevisiae mutants perturbed in nuclear pore complex assembly. BMC genetics. 17, 117.

Saitoh, H., Pu, R., Cavenagh, M., \& Dasso, M. (1997). RanBP2 associates with Ubc9p and a modified form of RanGAP1. Proceedings of the National Academy of Sciences of the United States of America, 94(8), 3736-3741. https://doi.org/10.1073/pnas.94.8.3736

Sakin, V., Richter, S. M., Hsiao, H. H., Urlaub, H., \& Melchior, F. (2015). Sumoylation of the GTPase Ran by the RanBP2 SUMO E3 ligase complex. Journal of Biological Chemistry, 290(39), 23589-23602. https://doi.org/10.1074/jbc.M115.660118

Salina, D., Enarson, P., Rattner, J. B., \& Burke, B. (2003). Nup358 integrates nuclear envelope breakdown with kinetochore assembly. Journal of Cell Biology, 162(6), 9911001. https://doi.org/10.1083/jcb.200304080

Savas, J. N., Toyama, B. H., Xu, T., Yates, J. R., \& Hetzer, M. W. (2012). Extremely longlived nuclear pore proteins in the rat brain. Science, 335(6071), 942. https://doi.org/10.1126/science.1217421

Schlenstedt, G., \& Solsbacher, J. (1999). Transport between the cytoplasm and the nucleus. Protoplasma, 209(3-4), 166-172. https://doi.org/10.1007/BF01453445

Shah, S., Tugendreich, S., \& Forbes, D. (1998). Major binding sites for the nuclear import receptor are the internal nucleoporin Nup153 and the adjacent nuclear filament protein Tpr. Journal of Cell Biology, 141(1), 31-49. https://doi.org/10.1083/jcb.141.1.31

Splinter, D., Tanenbaum, M. E., Lindqvist, A., Jaarsma, D., Flotho, A., Yu, K. Lou, Grigoriev, I., Engelsma, D., Haasdijk, E. D., Keijzer, N., Demmers, J., Fornerod, M., Melchior, F., Hoogenraad, C. C., Medema, R. H., \& Akhmanova, A. (2010). Bicaudal D2, dynein, and kinesin-1 associate with nuclear pore complexes and regulate centrosome and nuclear positioning during mitotic entry. PLoS Biology, 8(4). https://doi.org/10.1371/journal.pbio.1000350

Stade, K., Ford, C. S., Guthrie, C., \& Weis, K. (1997). Exportin 1 (Crm1p) is an essential 
nuclear export factor. Cell, 90(6), 1041-1050. https://doi.org/10.1016/S00928674(00)80370-0

Strawn, L. A., Shen, T., \& Wente, S. R. (2001). The GLFG Regions of Nup116p and Nup100p Serve as Binding Sites for Both Kap95p and Mex67p at the Nuclear Pore Complex. Journal of Biological Chemistry, 276(9), 6445-6452. https://doi.org/10.1074/jbc.M008311200

Ström, A. C., \& Weis, K. (2001). Importin- $\beta$-like nuclear transport receptors. Genome Biology, 2(6), 1-9. https://doi.org/10.1186/gb-2001-2-6-reviews3008

Sun, Q., Chen, X., Zhou, Q., Burstein, E., Yang, S., \& Jia, D. (2016). Inhibiting cancer cell hallmark features through nuclear export inhibition. Signal Transduction and Targeted Therapy, 1(May), 34-36. https://doi.org/10.1038/sigtrans.2016.10

Tamura, K. and H.-N. I. (2013). In Posidonia oceanica cadmium induces changes in DNA methylation and chromatin patterning. Journal of Experimental Botany, 64(4), 823-832. https://doi.org/10.1093/jxb/err313

Terry, L. J., \& Wente, S. R. (2009). Flexible gates: Dynamic topologies and functions for FG nucleoporins in nucleocytoplasmic transport. Eukaryotic Cell, 8(12), 1814-1827. https://doi.org/10.1128/EC.00225-09

Thakar, K., Karaca, S., Port, S. A., Urlaub, H., \& Kehlenbach, R. H. (2013). Identification of CRM1-dependent nuclear export cargos using quantitative mass spectrometry. Molecular and Cellular Proteomics, 12(3), 664-678. https://doi.org/10.1074/mcp.M112.024877

Toyama, B. H., Savas, J. N., Park, S. K., Harris, M. S., Ingolia, T., Yates, J. R., \& Hetzer, M. W. (2013). Identification of long-lived proteins reveals exceptional stability of essential cellular structures. Cell. 154(5), 971-982. https://doi.org/10.1016/j.cell.2013.07.037.Identification

Ulrich, A., Partridge, J. R., \& Schwartz, T. U. (2014). The stoichiometry of the nucleoporin 62 subcomplex of the nuclear pore in solution. Molecular Biology of the Cell, 25(9), 1484-1492. https://doi.org/10.1091/mbc.E13-12-0745

Ungricht, R., \& Kutay, U. (2017). Mechanisms and functions of nuclear envelope remodelling. Nature Reviews Molecular Cell Biology, 18(4), 229-245. https://doi.org/10.1038/nrm.2016.153

van Deursen, J., Boer, J., Kasper, L., \& Grosveld, G. (1996). G2 arrest and impaired nucleocytoplasmic transport in mouse embryos lacking the proto-oncogene CAN/Nup214. The EMBO Journal, 15(20), 5574-5583. https://doi.org/10.1002/j.1460- 
2075.1996.tb00942.x

Vollmer, B., Lorenz, M., Moreno-Andrés, D., Bodenhöfer, M., De Magistris, P., Astrinidis, S. A., Schooley, A., Flötenmeyer, M., Leptihn, S., \& Antonin, W. (2015). Nup153 Recruits the Nup107-160 Complex to the Inner Nuclear Membrane for Interphasic Nuclear Pore Complex Assembly. Developmental Cell, 33(6), 717-728. https://doi.org/10.1016/j.devcel.2015.04.027

Vollmer, B., Schooley, A., Sachdev, R., Eisenhardt, N., Schneider, A. M., Sieverding, C., Madlung, J., Gerken, U., MacEk, B., \& Antonin, W. (2012). Dimerization and direct membrane interaction of Nup53 contribute to nuclear pore complex assembly. EMBO Journal, 31(20), 4072-4084. https://doi.org/10.1038/emboj.2012.256

Von Appen, A., Kosinski, J., Sparks, L., Ori, A., DiGuilio, A. L., Vollmer, B., Mackmull, M. T., Banterle, N., Parca, L., Kastritis, P., Buczak, K., Mosalaganti, S., Hagen, W., AndresPons, A., Lemke, E. A., Bork, P., Antonin, W., Glavy, J. S., Bui, K. H., \& Beck, M. (2015). In situ structural analysis of the human nuclear pore complex. Nature, 526(7571), 140-143. https://doi.org/10.1038/nature15381

Walther, Tobias C., Pickersgill, H. S., Cordes, V. C., Goldberg, M. W., Allen, T. D., Mattaj, I. W., \& Fornerod, M. (2002). The cytoplasmic filaments of the nuclear pore complex are dispensable for selective nuclear protein import. Journal of Cell Biology, 158(1), 63-77. https://doi.org/10.1083/jcb.200202088

Walther, Toblas C., Askjaer, P., Gentzel, M., Habermann, A., Griffiths, G., Wilm, M., Mattaj, I. W., \& Hetzer, M. (2003). RanGTP mediates nuclear pore complex assembly. Nature, 424(6949), 689-694. https://doi.org/10.1038/nature01898

Weberruss, M., \& Antonin, W. (2016). Perforating the nuclear boundary - how nuclear pore complexes assemble. Journal of Cell Science, 129(24), 4439-4447. https://doi.org/10.1242/jcs. 194753

Wen, W., Meinkotht, J. L., Tsien, R. Y., \& Taylor, S. S. (1995). Identification of a signal for rapid export of proteins from the nucleus. Cell, 82(3), 463-473. https://doi.org/10.1016/0092-8674(95)90435-2

Werner, A., Flotho, A., \& Melchior, F. (2012). The RanBP2/RanGAP1 *SUMO1/Ubc9 Complex Is a Multisubunit SUMO E3 Ligase. Molecular Cell, 46(3), 287-298. https://doi.org/10.1016/j.molcel.2012.02.017

Weterman, M. A. J., Van Groningen, J. J. M., Tertoolen, L., \& Van Kessel, A. G. (2001). Impairment of MAD2B-PRCC interaction in mitotic checkpoint defective $\mathrm{t}(\mathrm{X} ; 1)$-positive renal cell carcinomas. Proceedings of the National Academy of Sciences of the United 
States of America, 98(24), 13808-13813. https://doi.org/10.1073/pnas.241304198

Winey, M., Yarar, D., Giddings, T. H., \& Mastronarde, D. N. (1997). Nuclear pore complex number and distribution throughout the Saccharomyces cerevisiae cell cycle by threedimensional reconstruction from electron micrographs of nuclear envelopes. Molecular Biology of the Cell, 8(11), 2119-2132. https://doi.org/10.1091/mbc.8.11.2119

Wolf, C., \& Mofrad, M. R. K. (2008). On the octagonal structure of the nuclear pore complex: Insights from coarse-grained models. Biophysical Journal, 95(4), 2073-2085. https://doi.org/10.1529/biophysj.108.130336

Wu, J., Matunis, M.J., Kraemer, D., Blobel, G., Coutavas, E. (1995). Nup358, a cytoplasmically exposed nucleoporin with peptide repeats, Ran-GTP binding sites, zinc fingers, a cyclophilin A homologous domain, and a leucine-rich region. The Journal of Biological Chemistry, 270(23), 14209-14214.

Xu, D., Grishin, N. V., \& Chook, Y. M. (2012). NESdb: A database of NES-containing CRM1 cargoes. Molecular Biology of the Cell, 23(18), 3673-3676. https://doi.org/10.1091/mbc.E12-01-0045

Xu, D., Marquis, K., Pei, J., Fu, S. C., Całatay, T., Grishin, N. V., \& Chook, Y. M. (2015). LocNES: A computational tool for locating classical NESs in CRM1 cargo proteins. Bioinformatics, 31(9), 1357-1365. https://doi.org/10.1093/bioinformatics/btu826 Yavuz, S., Santarella-Mellwig, R., Koch, B., Jaedicke, A., Mattaj, I. W., \& Antonin, W. (2010). NLS-mediated NPC functions of the nucleoporin Pom121. FEBS Letters, 584(15), 3292-3298. https://doi.org/10.1016/j.febslet.2010.07.008

Yoshida, K., Seo, H. S., Debler, E. W., Blobel, G., \& Hoelz, A. (2011). Structural and functional analysis of an essential nucleoporin heterotrimer on the cytoplasmic face of the nuclear pore complex. Proceedings of the National Academy of Sciences of the United States of America, 108(40), 16571-16576. https://doi.org/10.1073/pnas.1112846108

Yuh, M. C., \& Blobel, G. (2001). Karyopherins and nuclear import. Current Opinion in Structural Biology, 11(6), 703-715. https://doi.org/10.1016/S0959-440X(01)00264-0 Zuccolo, M., Alves, A., Galy, V., Bolhy, S., Formstecher, E., Racine, V., Sibarita, J. B., Fukagawa, T., Shiekhattar, R., Yen, T., \& Doye, V. (2007). The human Nup107-160 nuclear pore subcomplex contributes to proper kinetochore functions. EMBO Journal, 26(7), 1853-1864. https://doi.org/10.1038/sj.emboj.7601642

Zwerger, M., Eibauer, M., \& Medalia, O. (2016). Insights into the gate of the nuclear pore complex. Nucleus, 7(1), 1-7. https://doi.org/10.1080/19491034.2015.1130197 


\section{List of figures}

Figure 1: The arrangement of the nuclear pore complex (NPC) 14

Figure 2: Domains of Nup214 17

Figure 3: Schematic representation of the Ran cycle $\quad 20$

Figure 4: Nucleocytoplasmic transport $\quad 23$

Figure 5: Nup214 is mainly localized at the NE $\quad \mathbf{6 8}$

Figure 6: Different domains of Nup214 show different localizations

Figure 7: Inhibition of CRM1 export by LMB causes Nup214 to localize in the nucleus $\mathbf{7 1}$

Figure 8: Nup214 NES is highly conserved across vertebrates $\quad \mathbf{7 2}$

Figure 9: A mutation in Nup214 NES sequence leads to inhibition of Nup214 export $\quad \mathbf{7 3}$

Figure 10: An LMB resistant CRM1 can rescue Nup214 in transfected cells $\quad \mathbf{7 4}$

Figure 11: An isolated Nup214 coiled-coil fragment can localize to the nucleus under LMB

Figure 12: Different Nup214 NES containing fragments can form an export complex in a Ran dependent manner

Figure 13: A mutated Nup214 NES peptide fails to form a Ran dependent export complex $\mathbf{7 8}$

Figure 14: Concept of the fluorescence polarization assay $\quad \mathbf{8 0}$

Figure 15: Nup214 NES has a high affinity for CRM1 compared to other NESs $\quad \mathbf{8 1}$

Figure 16: A mutated Nup214 NES peptide cannot compete for CRM1 binding sites $\quad \mathbf{8 2}$

Figure 17: The Nup214 complex is stable in gel filtration $\quad \mathbf{8 3}$

Figure 18: LMB does not affect Nup88 localization $\quad \mathbf{8 4}$

Figure 19: Mutation in the NES region of Nup214 affect the interaction of Nup214 and Nup88

Figure 20: Mutation in the NES region of Nup214 shifts the localization of overexpressed

Nup88 to the nucleus $\quad \mathbf{8 6}$

Figure 21: GFP-Nup214 (680-1209) and GFP-Nup214 (680-1209)_L878A/L882A can immunoprecipitate Nup88 from cell lysate

Figure 22: An artificial NES sequence can rescue the phenotype of Nup214 with mutations in the NES

Figure 23: An artificial NES sequence localizes overexpressed GFP-Nup214 (1916-2033) to the cytoplasm

Figure 24: Depletion of Nup358 leads to loss of the Nup214 signal at the nuclear envelope 90

Figure 25: GFP-Nup214 is integrated in the nuclear envelope

Figure 26: Proximity ligation assays to monitor the proximity of CRM1 and Nup214 or Nup358, respectively

Figure 27: Overexpression of Nup214 restores Nup88 in Nup214 depleted cells $\quad 96$

Figure 28: Models of Nup214 NES function $\quad \mathbf{1 0 4}$

Figure 29: Characterization of goat ant-CRM1 antibody. CRM1 antibody was purified from goat serum

Figure 30: Characterization of rabbit anti-Nup214 antibody in vitro. Nup214 antibody was purified from rabbit serum

Figure 31: Characterization of cell lines stably expressing GFP-Nup214 (L878A/L882A) 133

Figure S1: An artificial NES sequence can sequesters CRM1 to the NE 131 


\section{List of tables}

Table 1: Equipment 32

Table 2: Software $\quad 33$

Table 3: Consumable $\quad 33$

Table 4: Kit $\quad 34$

Table 5: Enzyme $\quad 34$

Table 6: Table $\quad 34$

Table 7: Bacterial media $\quad 36$

Table 8: Stock solution $\quad 36$

$\begin{array}{ll}\text { Table 9: Buffers } & 37\end{array}$

Table 10: Mammalian cell lines $\quad 38$

Table 11: Bacterial strains $\quad 38$

Table 12: Primary antibodies $\quad 38$

Table 13: Secondary antibodies

Table 14: Synthesized peptides $\quad 40$

Table 15: Oligonucleotide for cloniing $\quad 40$

Table 16: Oligonucleotide for mutagenesis $\quad 42$

Table 17: Oligonucleotide for sequencing $\quad 42$

$\begin{array}{ll}\text { Table 18: siRNA } & 43\end{array}$

Table 19: Vectors $\quad 43$

Table 20: Available plasmids 44

Table 21: Generated plasmids 44 


\section{Appendix}

\section{Characterization of goat anti-CRM1 antibody}

Serum was collected and purified for anti-CRM1 antibodies. Different approaches were used to test and validate the efficiency of the antibody. As shown in figure $\mathrm{x}$, the antibody was concentrated to $0.1 \mathrm{mg} / \mathrm{ml}$. Cell lysates were collected and analyzed by SDS-PAGE and Western blotting, detecting CRM1 using different concentrations of the goat anti-CRM1 antibody. A dilution of 1:2000 was determined as a suitable concentration for detecting CRM1 from a cell lysate (figure $\mathrm{x}$ ). For further testing, different concentrations of the purified recombinant CRM1-His protein were analyzed by SDS-PAGE and Western blotting. As shown in figure $\mathrm{x}$, the CRM1 antibody could detect as little as $50 \mathrm{ng}$ of purified CRM1-His. These results confirm that the purified antibody was sensitive for detection of CRM1 protein. Finally, the efficiency of the antibody was validated using fixed cells. By probing fixed cells with different concentrations of CRM1 antibody, CRM1 protein was detected within the cells at a dilution of 1:500 (figure $\mathrm{x}$ ). These results validate our purified CRM1 antibody as being functional and can thus be further used for detection of CRM1 in different experimental setups. 
a

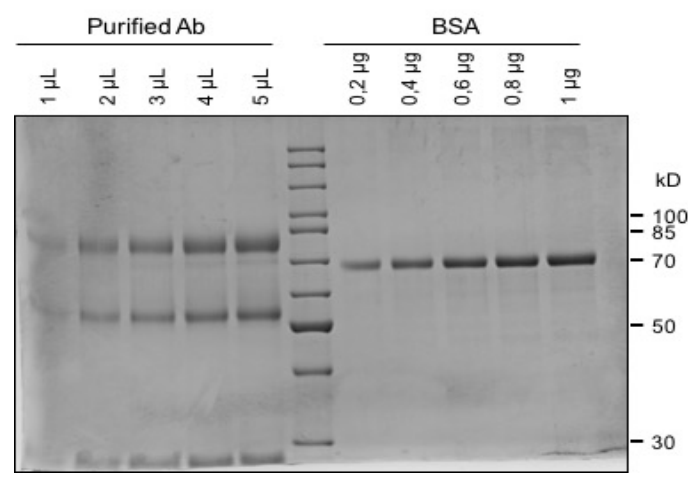

b

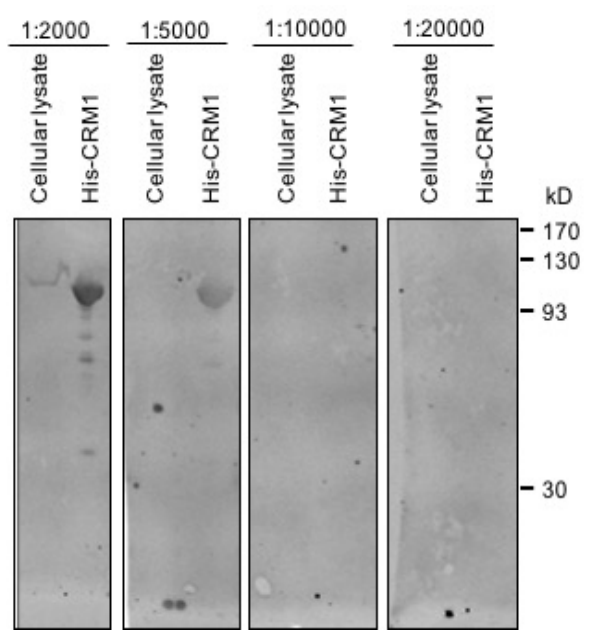

C

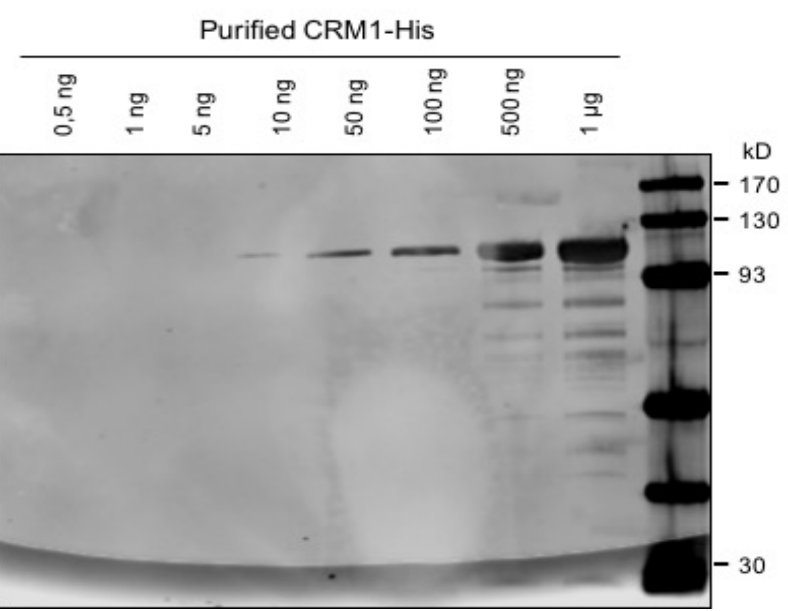

d

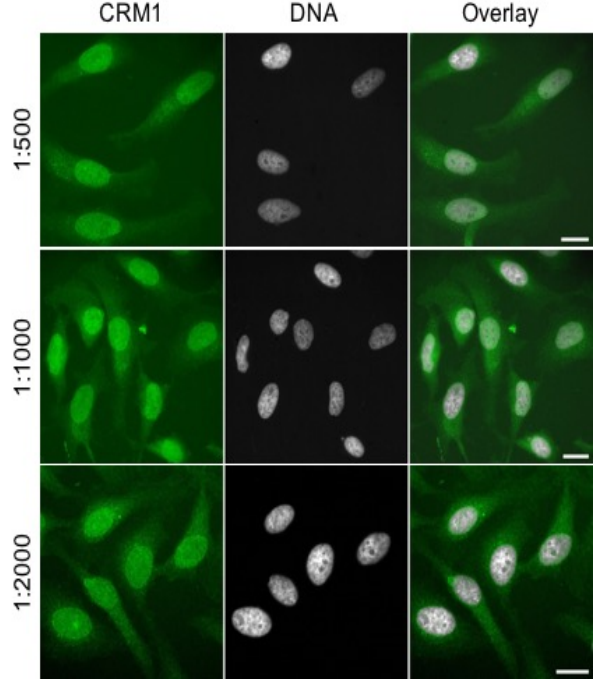

Figure 29: Characterization of goat ant-CRM1 antibody. CRM1 antibody was purified from goat serum. (A) The concentration of the purified CRM1 antibody was measured against bovine serum albumin (BSA). Different volumes of the purified CRM1 antibody were analyzed against different concertation of BSA using 10\% SDS-PAGE, as indicated. (B) 100 ug of whole cell lysate and different amounts of purified recombinant CRM1 protein were analyzed by 10\% SDS-PAGE and western blotting. The membranes were probed by various dilutions of the purified CRM1 antibody, as indicated. (C) Increasing amounts of recombinant purified CRM1-His protein were analyzed by $10 \%$ SDS-PAGE and Western blotting. A fixed concentration $(1: 2000)$ of the purified CRM1 antibody was used to probe the membrane to determine the minimum protein concentration amount? that can be detected with the antibody. (D) Different dilutions of the purified CRM1 antibody were used to stain endogenous CRM1 in fixed cells. The cells were immunostained by the purified CRM1 antibody by indirect immunofluorescence. The scale bar corresponds to $10 \mu \mathrm{m}$. (Figures obtained from Floriane Lagadec)

\section{Characterization of rabbit anti-Nup214 antibody}

Rabbit anti-Nup214 serum was collected and used for purification of anti-Nup214 antibodies. Several fractions containing the purified antibody were eluted and concentrated to $0.1 \mathrm{mg} / \mathrm{ml}$. Cell lysates were collected and analyzed by SDS-PAGE and Western blotting, detecting Nup214 using different concentrations of the purified antibody (figure 30a). The purified antibody detected Nup214 at a dilution of as low as 1:1000 (figure 30b). Cell lysates from Nup214-depleted cells, control cells and untreated cells were collected and analyzed by SDSPAGE and Western blotting using the purified antibody. As shown in figure 30c, Nup214 was 
successfully depleted in two different cell lines and our antibody was able to detect Nup214 in control cells but not the cells with Nup214 knockdown. These results confirm specificity of the purified antibody for Nup214. Finally, the efficiency of the purified anti-Nup214 antibody was validated in fixed cells. Different blocking agents, 1\% BSA, 3\% FCS and 0.2\% fish gelatin were used. Additionally, different dilutions of the purified Nup214 antibody were tested. As shown in figure $\mathrm{x}$, we could successfully detect Nup214 protein within the cells at a dilution of 1:500. Additionally, blocking the cells with 1\% BSA yielded the best results since a nuclear rim was detected in HeLa P4 cells in comparison to FCS and fish gelatin (figure 30c). Finally, COS7 cells showed a nuclear staining for Nup214 similar to HeLa P4 cells. The Nup214 localization in COS7 is similar to previously published results showing similar staining.
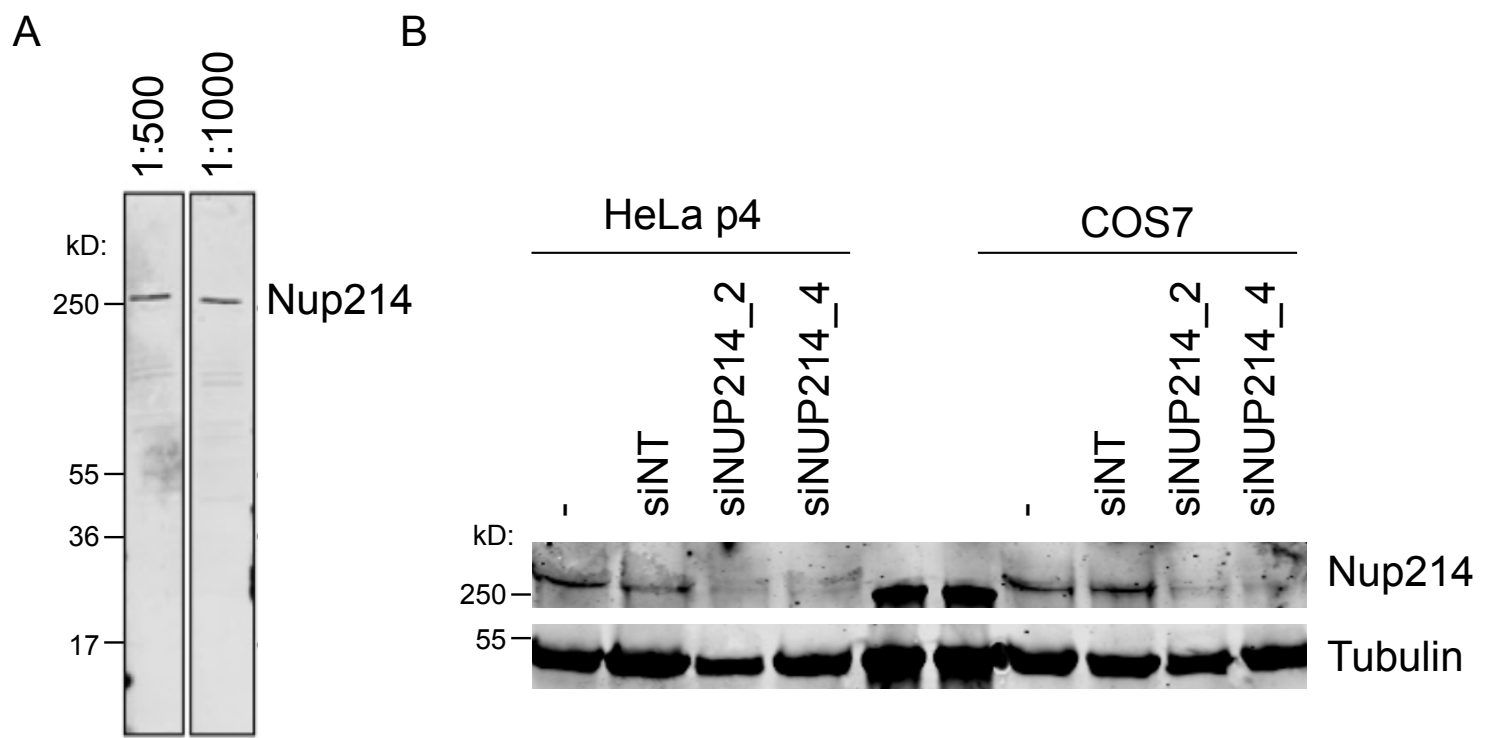
$\mathrm{c}$
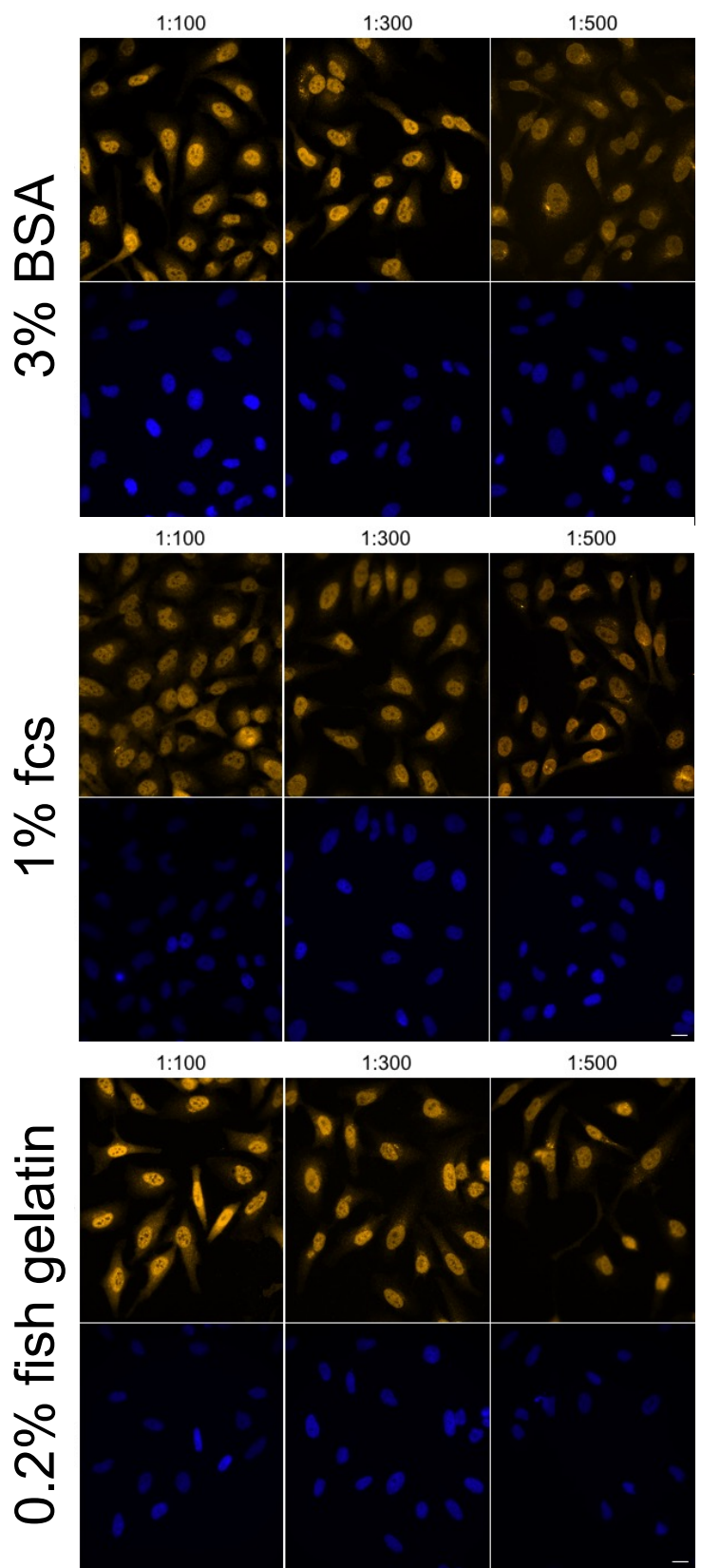

\section{$\cos 7$}
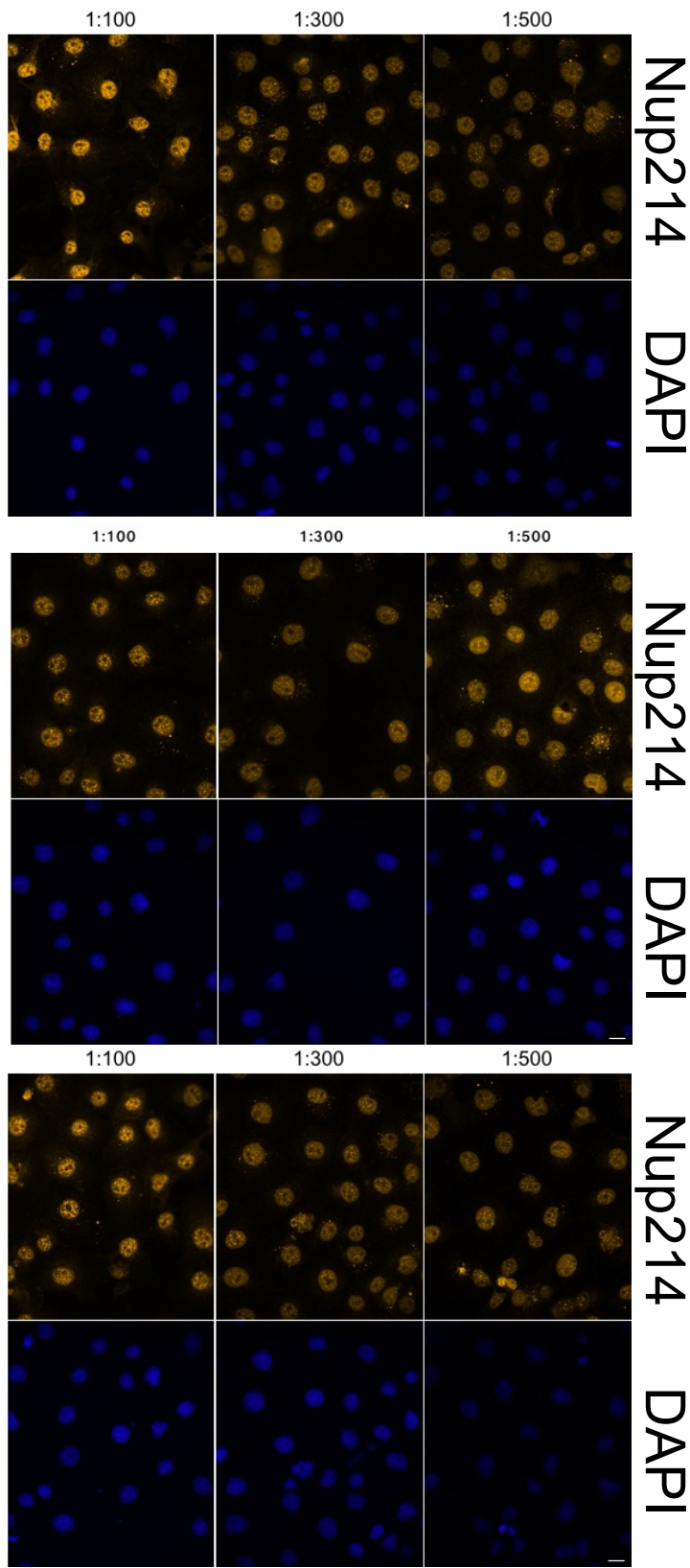

Figure 30: Characterization of rabbit anti-Nup214 antibody in vitro. Nup214 antibody was purified from rabbit serum. (A) 100 ug of HeLa P4 whole cell lysates were analyzed by SDS-PAGE and Western blotting. The membranes were probed with various dilutions of the purified Nup214 antibody, as indicated. (B) Nup214 was depleted in HeLa P4 and COS7 cells using two different siRNAs, as indicated. As a control, the cells were transfected with a mock siRNA. Cell lysates from Nup214-depleted cells and control cells were collected and 100 ug of proteins were analyzed by SDS-PAGE and Western blotting, detecting Nup214 using a 1:500 dilution of the purified Nup214 antibody. Tubulin was used as a loading control to ensure equal loading of proteins.

\section{Characterization of cell lines stably expressing GFP-Nup214}

\section{(L878A/L882A)}

Inducible expression of GFP-Nup214 (L878A/L882A) in Hek Flp-In cells was controlled by increasing concentrations of tetracycline $(0.1-1 \mu \mathrm{g} / \mathrm{ml})$. Cell were analyzed by confocal 
microscopy, detecting for GFP. As shown in figure 31, tetracycline induced the expression of GFP-Nup214 (L878A/L882A) at concentrations as low as $0.1 \mu \mathrm{g} / \mathrm{ml}$.

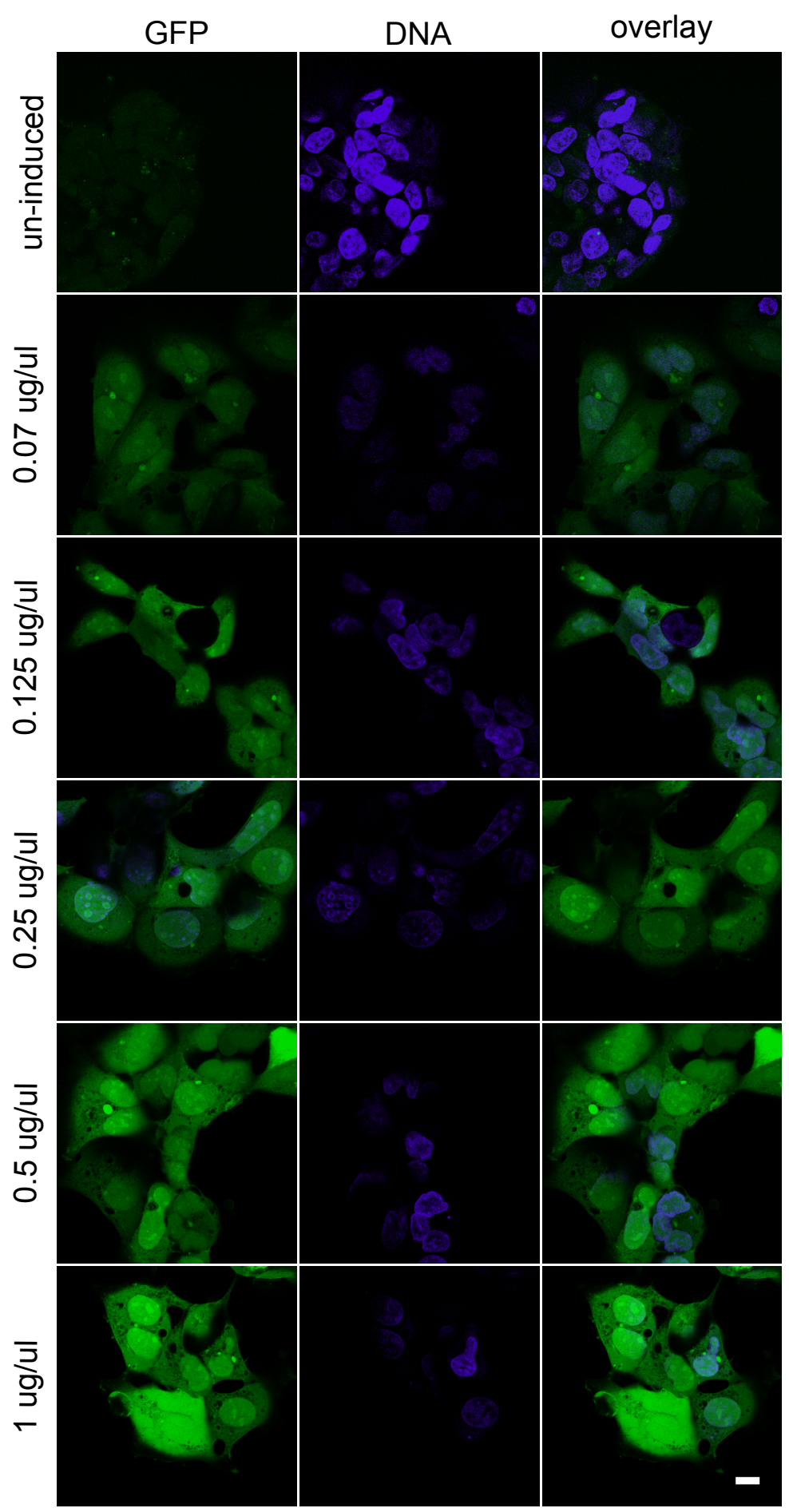

Figure 31: Characterization of cell lines stably expressing GFP-Nup214 (L878A/L882A). Hek293-Flp-In were induced using different concentrations of tetracycline as indicated and analyzed by confocal microscopy, detecting for GFP. The scale bar corresponds to $10 \mu \mathrm{m}$. 


\section{Supplementary figures}

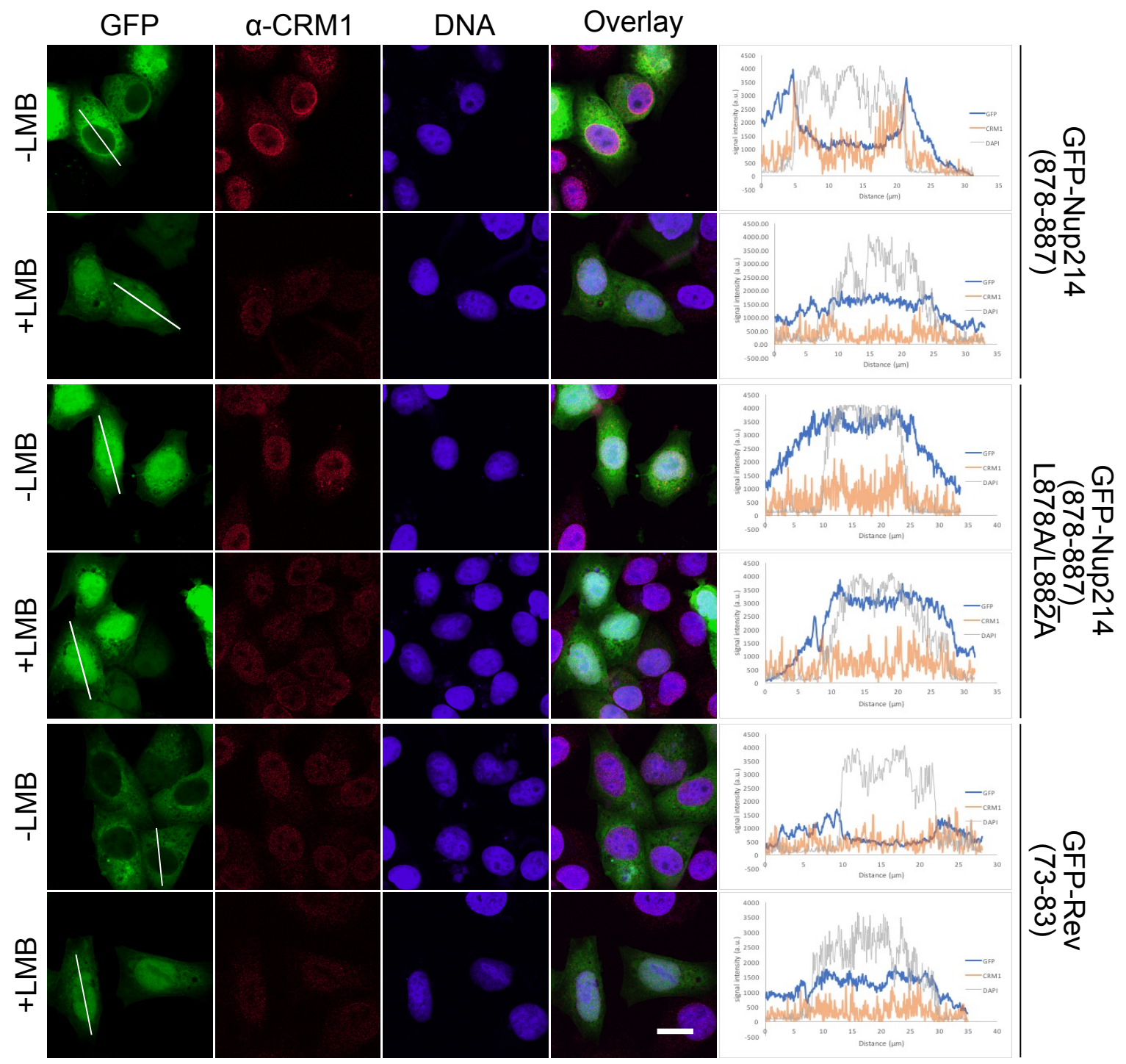

Figure 32: An artificial NES sequence can sequesters CRM1 to the NE. Either the NES of Nup214 or HIV-1 Rev was tagged with GFP. HeLa P4 cells were transfected with plasmids coding for GFP-Nup214 (878-887), GFP-Nup214 (878887)_L878A/L882A and GFP-Rev (73-83). Cells were treated with or without $10 \mathrm{nM} \mathrm{LMB}$ for 2 hours, as indicated. A line was drawn across each cell and the average intensities of five cells from each condition was plotted against the distance of the line on the $\mathrm{x}$-axis shown in um. The scale bar corresponds to $10 \mu \mathrm{m}$. 


\section{Abbreviation}

${ }^{\circ} \mathrm{C}$

$\alpha$

a.a.

$\beta$

$\mu \mathrm{m}$

$\mu \mathrm{M}$

AP

APS

ATP

bp

BSA

C-terminus

CRM1

DMEM

DMSO

DNA

dNTPs

DTT

E.Coli

EDTA

ER

EtOH

FG-repeat

$\mathrm{g}$

GAP

GAPDH

GDP

GFP

GST

GTP

H. sapiens

HA

$\mathrm{HCl}$

HEPES

His

HIV

IF

IPTG

$\mathrm{kDa}$

$\mathrm{K}_{\mathrm{dS}}$

LB degree Celsius

anti- (for antibodies) or alpha

Amino acid

beta

micometer

micromolar

Aprotinin

Ammonium persulfate

adenosine-5-triphosphate

Base pair

Bovine serum albumin

Carboxy

chromosome region maintenance 1

Dulbecco's modified eagles medium

dimethyl sulfoxide

Desoxyribonucleic acid

2'-desoxynucleoside-5'-triphosphate

dithiothreitol

Escherichia coli

ethylenediaminetetraacetic acid

endoplasmic reticulum

ethanol

phenylalanine glycine repeat

G-force

GTPase-activating protein

Glyceraldehyde 3-phosphate dehydrogenase

guanosine-5' -diphosphate

green fluorescent protein

glutathione-S-transferase

guanosine-5' -triphosphate

Homo sapiens

hemagglutinin

hydrochloric acid

2-[4-(2-hydroxyethyl)piperazin-1-yl]ethanesulfonic acid histidine tag

human immunodeficiency virus

immunofluorescence

isopropyl-beta-D-thiogalactopyranoside

kilo Dalton

dissociation constant

Luria-Bertani 
LMB

M. musculus

MBP

$\mathrm{mg}$

$\mathrm{ml}$

$\mathrm{mM}$

MVM

$\mathrm{N}$-terminus

NE

NES

NFAT

NLS

$\mathrm{nm}$

$\mathrm{nM}$

NPC

nt

Nup

PAGE

PBS

PCR

PLA

PMSF

Ran

RanGAP1

RanGEF

RCC1

RFP

RNA

rpm

S. cerevisiae

SDS

siRNA

SPN1

SV40

TAE

tev

Triton X-100

Tween 20

UV

$\mathrm{v} / \mathrm{v}$

$\mathrm{w} / \mathrm{v}$

wt
Leptomycin B

Mus musculus

maltose binding protein

milligram

milliliter

millimolar

minute virus of mice

amino terminus

Nuclear envelope

nuclear export signal

nuclear factor of activated T-cells

nuclear localization signal

nanometer

nanomolar

Nuclear pore complex

Non-targeting

Nucleoporin

polyacrylamide gel electrophoresis

phosphate buffered saline

polymerase chain reaction

proximity ligation assay

phenylmethylsulphonyl fluoride

Ras-related nuclear protein

RanGTPase-activating protein

Ran guanine nucleotide exchange factor

Regulator of chromatin condensation 1

red fluorescent protein

ribonucleic acid

rotations per minute

Saccharomyces cerevisiae

sodium dodecyl sulphate

small interfering RNA

snurportin 1

Simian virus 40

Tris/ Acetate/ EDTA

TEV-protease cleavage site

4-octylphenol polyethoxylate

polyoxyethylene (20) sorbitan monolaurate

ultraviolet

volume per volume

weight per volume

wildtype 


\section{Acknowledgment}

I would like to thank Professor Dr. Ralph Kehlenbach for giving me the opportunity to do my $\mathrm{PhD}$ work in his lab and for the continuous support over the course of the PhD.

I would like to thank the members of my thesis committee Prof. Heike Krebber and Dr. Achim Dickmanns for the helpful discussions and inputs to my project.

I thank all current members of my lab (Dr. Imke Baade, Dr. Birgit Caspar, Christina James, Floriane Lagadec and Marius Pörschke) and former members (Dr. Marret Müller, Christiane Spillner, Dr. Marina Blenski and Dr. Janine Pfaff) of the lab, for the help provided over the course of this PhD. Special thanks to Dr. Imke Baade, Dr. Sujoy Bera and Christina James for proof-reading my thesis and for the useful feedback.

I also thank all current and previous members of the department of molecular biology, members of Blanche Schwappach's lab, Markus Bohnsack's lab and Ruth Geiss-Friedlander's lab, for contributing to a nice working environment within the inistitue and also out of the institute.

I want to thank our collaborators in the lab of Professor Dr. Ralf Ficner, special thanks for Dr. Thomas Monecke and Alaa Shaikhqassem for their help with teaching some of the techniques used in this thesis as well as supplying some material that was used for some experiments.

I want to thank everyone who was of support for me during these previous years of my $\mathrm{PhD}$.

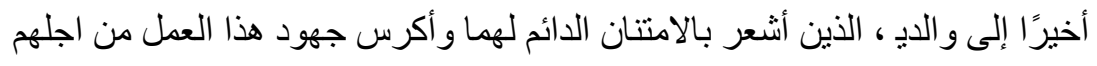




\section{Curriculum vitae}

\section{Personal information}

Name

Date of Birth

Place of Birth

Nationality
Mohamed Hamed

25.04.1990

Cairo

Egyptian

\section{Education}

08/2016 - 05/2020 PhD student, George-August-Universität Göttingen

Group of Prof. Dr. Ralph H. Kehlenbach

Department of Molecular Biology,

University Clinic Göttingen

$\mathrm{PhD}$ Thesis title:

"A nuclear export sequence in Nup214 promotes its targeting to the nuclear pore complex"

10/2013 - 12/2015 Master of Biomedical Sciences, Bonn-Rhein Sieg University of Applied Sciences

Master Thesis in the group of Prof. Dr. Sven Perner Department of Prostate Cancer, University of Bonn

Master Thesis title:

"Role of Estrogen Related Receptor-alpha (EsRRa) in progress of prostate cancer"

09/2007 - 07/2012 Bachelor of Pharmacy, Modern Sciences and Arts University, Cairo/ University of Greenwich. London 
